\title{
Modeling the biophysical mechanisms of sound encoding at inner hair cell ribbon synapses
}

\author{
Dissertation \\ for the award of the degree \\ Doctor rerum naturalium \\ of the Georg August University Göttingen
}

submitted by

Nikolai M. Chapochnikov

From Moscow, Russia

Göttingen, November 2011 



\section{Examination committee}

\section{Prof. Dr. Fred Wolf}

Research Group Theoretical Neurophysics

Department of Nonlinear Dynamics

Max Planck Institute for Dynamics and Self-Organization, Göttingen

Reviewer, Member of the thesis Committee

\section{Prof. Dr. Tobias Moser}

InnerEarLab

Department of Otolaryngology

University of Göttingen School of Medicine

Reviewer, Member of the thesis Committee

\section{Dr. Alexander Gail}

Sensorimotor Group

German Primate Center, Göttingen

Member of the thesis Committee

\section{Prof. Dr. Erwin Neher}

Department of Membrane Biophysics

Max Planck Institute for Biophysical Chemistry, Göttingen

\section{Prof. Dr. Tim Gollisch}

Research Group Sensory Processing in the Retina

Department of Ophthalmology

University of Göttingen School of Medicine

\section{Dr. Andreas Neef}

Department of Nonlinear Dynamics

Max Planck Institute for Dynamics and Self-Organization, Göttingen

Date of oral examination: $15^{\text {th }}$ of December 2011 



\section{Affidavit}

I hereby ensure that the thesis has been written independently and with no other sources and aids than quoted.

Nikolai M. Chapochnikov

Göttingen, November 2011 



\section{Contents}

Acknowledgements $\quad$ iv

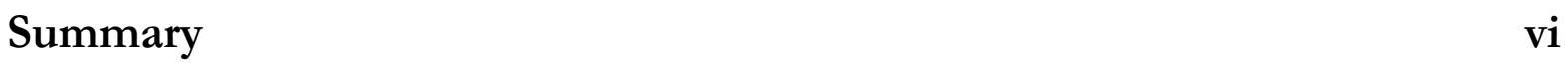

List of abbreviations viii

1 Introduction $\quad 2$

1.1 Action potentials in neurons 4

1.2 Synapses and synaptic transmission $\quad 7$

1.3 Overview of the peripheral auditory system $\quad 8$

1.4 Inner hair cell ribbon synapses $\quad 11$

1.5 Multiquantal release at ribbon synapses 14

1.6 Sound encoding by the auditory nerve fibers 15

1.7 A note on modeling 19

2 Spike encoding of neurotransmitter release timing by spiral ganglion neurons of the cochlea $\quad 20$

2.0 Abstract 21

2.1 Introduction $\quad 22$

2.2 Results $\quad 24$

2.2.1 IHC exocytosis evokes SGN spikes with great success 24

2.2.2 Influence of EPSP kinetics on action potential latency 26

2.2.3 Apical SGNs exhibit phasic responses and have a low rheobase 28

2.2.4 Effects of waveform kinetics and size on spike latency and jitter 31

2.2.5 Modeling the mechanism of spike generation in the SGN 35

2.2.6 EPSC-like stimulation and comparison to synaptically-evoked spikes 39

2.3 Discussion $\quad \mathbf{4 2}$

2.3.1 Fast and robust spiking at the origin of the auditory code 42

2.3.2 Phasic excitability of the spiral ganglion neuron 43

2.3.3 Synapse-spike initiation coupling 44

2.3.4 Consequences on SGN in vivo functional heterogeneity 45

2.4 Materials and Methods $\quad 46$

2.4.1 Preparation and electrophysiology 46

2.4.2 Data analysis 46

2.4.3 EPSC-like shapes $\quad 48$

2.4.4 Two-compartment model 48

2.4.5 Leaky integrate and fire models $\quad 50$ 
3 The synaptic ribbon as a Brownian conveyor trap

3.1 Introduction 53

3.2 Results $\quad 54$

3.2.1 Model of vesicle diffusing on the ribbon surface 54

3.2.2 High vesicle packing density immobilize vesicles 54

3.2.3 High vesicle packing on the synaptic ribbon enables efficient vesicle replenishment 55

3.3 Discussion $\quad 58$

3.4 Methods $\quad 59$

3.4.1 Simulation volume, and vesicle pool replenishment 59

$\begin{array}{ll}3.4 .2 & \text { Brownian motion of vesicles }\end{array}$

3.4.3 The diffusion coefficient 61

3.4.4 Vesicle repulsion forces 61

3.4.5 Boundary repulsion $\quad 62$

3.4.6 Exocytosis 62

3.4.7 Parameters summary used for simulations 63

4 Release at ribbon synapses: multiquantal, uniquantal or subquantal? 64

4.0 Abstract $\quad 65$

4.1 Introduction 66

4.2 Results 71

4.2.1 Coordination of vesicle release by $\mathrm{Ca}^{2+}$ nanodomain control of exocytosis

4.2.2 Simulation of $\mathrm{Ca}^{2+}$ coordinated exocytosis suggest a trade of efficiency for temporal precision $\quad 72$

4.2.3 Homotypic fusion model of MQR 77

4.2.4 $\mathrm{Ca}^{2+}$ independent homotypic fusion $\quad 78$

$\begin{array}{ll}\text { 4.2.5 } \mathrm{Ca}^{2+} \text { regulated homotypic fusion } & 78\end{array}$

4.2.6 Homotypic fusion dependent on $\mathrm{Ca}^{2+}$ and vesicle size 82

4.2.7 Uniquantal and subquantal release interpretation 84

\section{$\begin{array}{ll}4.3 \text { Discussion } & 87\end{array}$}

4.4 Methods $\quad 89$

4.4.1 $\mathrm{Ca}^{2+}$-triggered synchronized exocytosis model 89

4.4.2 Vesicle diffusion and homotypic fusion model 91

4.4.3 Glutamate diffusion and binding to the AMPAR receptors 95

4.4.4 Neurotransmitter escape from the synaptic vesicle through a fusion pore 96

$\begin{array}{ll}4.5 \text { Supplement } & \mathbf{9 7}\end{array}$

4.5.1 Note: homotypic fusion rate gradient from the active zone 97

4.5.2 Supplementary Figures and Tables 98 
5 Uniquantal and frustrated release at the inner hair cell ribbon synapse 106

$\begin{array}{ll}5.1 \text { Introduction } & 107\end{array}$

5.2 Results 108

5.2.1 Amplitude and charge of monophasic and multiphasic EPSCs 108

5.2.2 mEPSCs do not compose multiphasic EPSCs 110

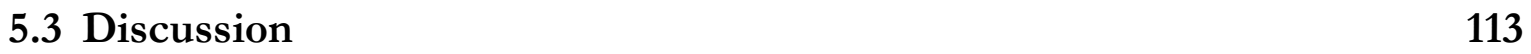

$\begin{array}{ll}5.4 \text { Methods } & 115\end{array}$

$6 \mathrm{Ca}^{2+}$ nanodomain control of exocytosis and its consequences on auditory $\begin{array}{ll}\text { nerve fiber response properties } & 118\end{array}$

6.1 Introduction 119

6.2 Results 121

6.2.1 $\mathrm{Ca}^{2+}$ sensor sensitivity and $\mathrm{Ca}^{2+}$ concentration at the IHC ribbon synapse

6.2.2 Different $\mathrm{Ca}^{2+}$ channel and vesicle positioning could account for auditory nerve fiber functional heterogeneity 123

6.2.3 Relation of exocytosis-voltage curves to the rate-level functions 125

6.2.4 Resolution-integration paradox in the auditory system 126

6.2.5 Phase locking: are 2 channels more precise than 40? 127

6.3 Discussion 129

6.4 Methods 130

$\begin{array}{lll}\text { 6.4.1 } \mathrm{Ca}^{2+} \text { channel model } & 130\end{array}$

6.4.2 Buffer environment and $\mathrm{Ca}^{2+}$ current 130

6.4.3 Microdomain regime of exocytosis 131

6.4.4 Nanodomain regime of exocytosis 131

$\begin{array}{lll}\text { 6.4.5 Vesicle cycle } & 131\end{array}$

7 Discussion 132

7.1 Fast synaptic transmission and role of the synaptic ribbon 132

7.2 $\mathrm{Ca}^{2+}$ nanodomain and microdomain control of exocytosis 134

7.3 Synapse sensitivity modulation with the number of $\mathrm{Ca}^{2+}$ channels 135

7.4 Vesicle fusion pore 135

$\begin{array}{ll}7.5 \text { Class III excitability } & 137\end{array}$

8 References 138 


\section{Acknowledgements}

I am immensely grateful to Fred Wolf and Tobias Moser, both who were my "Doktorvaters" during my doctorate studies. They gave me a very subtle mixture of supervision, guidance and independence, which was perfect for my evolution as a scientist. I thank them for showing me extremely fascinating and important problems to address, for giving me the example of what thoroughness and quality research means and for devoting their time to very valuable discussions. I thank them for teaching me to stay open, to not reach premature conclusions and to always seek for advice from experts. I am happy that I could always rely on them in important moments and specially thank them for making my research go smoothly and for caring for my scientific career in general. Finally I thank them for their comments on the writing.

I thank Alexander Gail for being part of my thesis committee and for giving advice on my research. It was always very helpful for me to have an external point of view on my research.

I thank Erwin Neher for being part of my extended thesis committee and giving me the opportunity to present my research in his laboratory on two occasions. I very much benefited from his knowledge, experience and advices.

I thank Tim Gollisch for being part of my extended thesis committee and the discussion about theoretical and experimental neurosciences.

I thank Andreas Neef for his guidance and for many scientific and meta-scientific discussions we had. His wisdom made me take the right strategic decisions at many stages during my doctoral studies and made me chose the right approaches. I also thank him for his great teaching abilities and patience while introducing me to single channel patch-clamp technique. I thank him for participating in all my thesis committee meetings and giving me insights on what to do and for being part of my extended thesis committee. I also thank him for sharing his intuition and teaching me how to think in more physiological ways.

I thank Thomas Frank for very rich and fruitful discussions. For his openness and for his biological intuition from which I learned enormously. Also for many discussions about how to do science and how to be productive. Finally, his success, perfectionism and efficiency where always an inspiration for me.

I was extremely lucky to be part of a very fruitful collaboration with Mark A. Rutherford. I was very inspired by his vision and methodology to achieve a desired goal. I learned from him about designing projects and bringing them to completion. He also taught me to set deadlines for my own work. I was happy that we could learn from each other to a great extend and from each other's ways of thinking and approaching science.

I thank Georg Martius for many discussions, for all his advice on programming, on running simulations on clusters and on Mathematica.

I thank Tina Pangrsic for many scientific discussions, for her wisdom and giving me important advices.

I thank Tatjana Tchumatchenko for valuable exchange of ideas, which helped me to structure my doctoral studies and to deal with some crucial aspects. As well for many non-scientific interactions.

I thank Alexander Egner and Alexander Meyer for our collaboration.

I thank Mantas Gabrielaitis for the beginning of a fruitful collaboration and for discussions. 
I thank all the members of the Max Planck Institute for Dynamics and Self-Organization as well as all members of the InnerEarLab for creating such a wonderful environment and for all possible scientific and non-scientific interactions. In particular: Aaron Wong, Wolfgang Keil, Jacob Neef, Christoph Kirst, Regis Nouvian, Michael Monteforte, Nicola Strenzke, Jakob Metzger, Iliana Panou, Olav Stetter, Alejandro Mendoza Schulz, Dmitry Tsigankov, Tzu-Lun Wang, Primoz Pirih, Sarah Helfmann, Ellen Reisinger, Kirsten Reuter, Andreas Brandt, Linda Hsu, Daniel Herde, Peter Hiemeyer, Carolin Wichmann, Maximilian Pouelma Touzel, Marc Timme, Anna Levina, Andres Agudelo-Toro, Harold Gutch, Dominika Lyzwa, Vitaly Belik, Anna Gehrt, Dmitry Bibichkov, Armin Biess, Raoul Martin Memmesheimer, Jan Nagler, Wei Wei, Hideki Takago, Darina Khimich, Tomoko Oshima-Takago, Victor Hernandez, Zhizi Jing, Maria Magdalena, Friederike Wolk, Anna Bulankina, Pinar Öz.

For creating a great and inspiring place to work at the MPI DS and for organizing the beautiful winter seminars I am very grateful to Theo Geisel.

For scientific discussions I acknowledge: Walter Stühmer, Manfred Lindau, Jim Hudspeth, Angus Silver, Josh Singer, William Roberts, Marcus Müller, Reinhard Jahn, Thorsten Hohage, Rob Shaw, Eve Marder.

For making everything work smoothly during the doctoral studies I would like to thank Brigitte Higazi, Yorck-Fabian Beensen, Gerhard Hoch, Tobias Niemann, Viktoryia Novak, Regina Wunderlich, Barbara Guichemer, Franciska Hudemann and Sandra Gerke.

Special thanks to the GGNB team, Kirsten Pöhlker, Christina Bach for all the courses, the organization of retreats and conferences, and for travel grants and for making administration during the doctoral studies as smooth as possible.

I express my deep gratitude to Devann Rutherford, who has been my office mate in the last years. It is hard to dream of a better place to work and a person to sit with in the same room. I thank her for all the good moments, her help and support in numerous instances and for a perfect joyful atmosphere.

I thank the city of Göttingen for being such an inspiring place for research.

I thank Iliana Panou for her support, her careful listening and understanding during these years.

I thank Derya Akad for her unlimited care and her encouragements and for being present in the final phase of my doctorate. Also I appreciate her comments on the writing.

I would like to thank friends in Göttingen who supported me during my doctorate: Kristina Saliy, Wolfgang Buchholz, Milutin Susnika, Detlef Koch, Christoph Solveen, Olav Stetter, Nora Wender, Ghazaleh Afshar, Felicitas Sedlmair, Kassir Aminou, Ulrich Vetter, Carsten Grabow, Victoria Shushakova, Stephanie Westendorff.

And also friends abroad: Gengis Ali Khan, Félicia Soumah, Mikhail Kibalchenko, Alexei Altoukhov, Philippe Labouchère, Tatjana Fleischmann, Nicolas Labau, Sheila Sabune, Melina Wilson, Salim Refas and Arnaud Salomon.

I thank my previous mentors Davor Pavuna and Hervé Kunz for inspiring me and believing in me.

Finally I immensely thank my family, my brother and especially my parents for their unlimited love, support, care and affection. I thank them for always believing in me, for sharing their experience and giving me uncountable number of crucial advices and caring about what is the best for me. Lastly I thank them for inspiring me to always do better. 


\section{Summary}

The fine temporal precision and the wide dynamic range of the auditory system are possible through the reliable performance of all of its components. A key stage in the auditory coding is the transformation of the analog and continuous electrical signal of the inner hair cells (IHCs) into the discrete spike timings in the auditory nerve fibers (ANFs). In this work, by using modeling, we investigated the biophysical specializations at the IHC ribbon synapse, which performs this transcoding of the auditory signal.

First, in combination with experiments, we studied the coupling of the ANF postsynaptic bouton to its action potential generator. We found that ANFs faithfully encode the precise timings of the neurotransmitter release from IHCs (chapter 2).

Then we investigated the sound signal encoding at the presynaptic site. By modeling vesicle diffusion on the synaptic ribbon, we suggest that the ribbon acts as a "Brownian conveyor trap", which accelerates vesicle arrival to the release sites (chapter 3). By enabling high vesicle replenishment rates, the ribbon might expand the range of possible exocytosis rate and thus broaden the coding capabilities of the synapse.

One specialization of ribbon synapses seems to be the ability to release multiple neurotransmitter quanta synchronously (MQR: synchronous multiquantal release). The MQR interpretation of the electrophysiological data was mostly influenced by the presence of unusually large "spontaneous" EPSCs (up to $800 \mathrm{pA}$ ) and of multiphasic EPSCs, which resemble the exocytosis of multiple poorly synchronized vesicles. The mechanism underlying MQR is however unclear. In chapter 4 we modeled the candidate biophysical scenarios of $\mathrm{MQR}$ and found that they were rather unlikely to take place, although regulated homotypic vesicle fusion might seem plausible. We suggested that alternative explanations of MQR should be considered. We found that one plausible scenario is that large EPSCs are uniquantal and that smaller EPSCs reflect subquantal release. Thus, large EPSCs might arise from a postsynaptic specialization (large neurotransmitter receptor clusters) rather than from a presynaptic one (many vesicles release at once). To test this hypothesis, in chapter 5 we analyzed postsynaptic bouton recordings of ANFs and deconvolved multiphasic EPSCs. The result of the deconvolution was more consistent with the interpretation of uniquantal release and flicking vesicle fusion pore rather than with poorly synchronized vesicle release.

In the last chapter (chapter 6), we investigated biophysical mechanisms at the IHC ribbon synapse that might account for several response properties of ANFs: in particular their functional heterogeneity and their coding capabilities. We found how different 
number of $\mathrm{Ca}^{2+}$ channels might underlie variability in the ANF dynamic range. Also we found that the control of vesicle exocytosis by only a few $\mathrm{Ca}^{2+}$ channels can be as precise as the control by many channels.

A short connecting summary introduces each chapter to facilitate reading. Each chapter can be read independently and long chapters (2 and 4) contain an abstract. 


\section{List of abbreviations}

AIS

AMPA

$\mathrm{ANF}$

AP

AZ

$\mathrm{CF}$

CV

$\mathrm{dB}$

EM

EPSC

EPSP

frog $\mathrm{HC}$

IHC

kB

mEPSC

MQR

MVR

PSD

PSF

quantal content

$\mathrm{RBC}$

RRP

s.d.

SGN

SPL axon initial segment

$\alpha$-amino-3-hydroxy-5-methyl-4-isoxazolepropionic acid

auditory nerve fiber, same as SGN

action potential, spike

active zone

characteristic frequency

coefficient of variation

decibel

electron microscopy

excitatory post-synaptic current

excitatory post-synaptic potential

bullfrog amphibian papilla hair cell

inner hair cell

Boltzmann constant $=1.3806503 \times 10^{-23} \mathrm{~m}^{2} \mathrm{~kg} \mathrm{~s}^{-2}$

miniature EPSC, containing only one neurotransmitter quanta

synchronous multiquantal release

multivesicular release

postsynaptic density

point spread function

the number of neurotransmitter quanta contained in an EPSC (or in a vesicle for compounds)

rod bipolar cell of the retina

ready releasable pool - vesicles at the active zone that are ready to be released.

standard deviation

spiral ganglion neuron, same as ANF

sound pressure level 



\section{Introduction}

The brain can be mostly viewed as a device integrating sense perceptions and transforming them into muscle actions. The cells performing these computations are neurons, which are connected via chemical and electrical synapses. The human brain contains around 100 billion neurons and 100 trillion synapses. Neurons are composed of dendrites, a soma and an axon (Fig. 1.1). The dendrites and soma integrate the excitatory and inhibitory input of presynaptic neurons, whereas the axon sends the results of the neurons computation to postsynaptic neurons. Neuronal membrane rests at a negative electrical potential and when enough excitatory input is received, the neurons excitable membrane generates an active depolarizing electrical wave, which is propagated from the soma to axons presynaptic terminals. Different types of ion channels situated on the neuron membrane mediate this electrical wave. Ion channel opening and closure is influenced by the transmembrane potential. Once the electrical depolarization reaches a presynaptic terminal, a chemical reaction elicited by the entry of calcium triggers the exocytosis of vesicles filled with neurotransmitter. The neurotransmitter is released into the synaptic cleft and binds to postsynaptic receptors, which generate a change in membrane potential in the postsynaptic neuron, thus propagating the neuronal signal.

Neurons are very diverse in form, function and response properties. They can form complex networks where each node has about 1000-10000 synaptic connections. Synapses also vary greatly in size, form and function throughout the brain.

In this thesis we will study biophysical mechanisms contributing to sound encoding at first synapse and the first neuron on the auditory pathway: the ribbon synapse in the inner hair cell and the spiral ganglion neuron. Both are different in comparison to usual cortex synapses and neurons. The ribbon synapses it is not part of a strictly speaking neuron, put of a graded potential cell (the inner hair cell), and it contains a large electron dense structure (the ribbon) to which synaptic vesicles tether. The spiral ganglion neuron, on the other hand, has only one presynaptic contact and few postsynaptic. In addition, both have also functional specializations, as we will see throughout this work.

In the next sections will we will shortly review the mechanisms of action potential generation and synaptic transmission (Kandel et al., 2000; Purves et al., 2001; Dayan and Abbott, 2004). Then we will focus on the auditory system (Gelfand, 2004; Moller, 2006), and specifically on the inner hair cell ribbon synapse. 


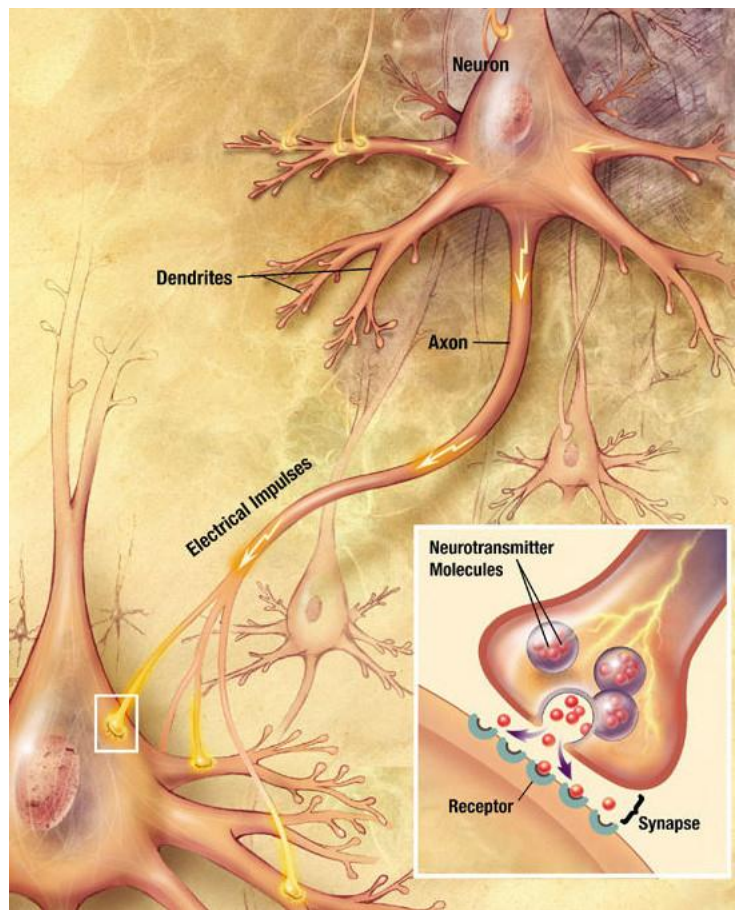

Figure 1.1 Scheme of a typical neuron and of a synaptic transmission Source : www.nia.nih.gov 


\subsection{Action potentials in neurons}

The negative membrane potential of neurons (approximately between $-80 \mathrm{mV}$ and -60 $\mathrm{mV}$ ) is due to different concentrations of charged ions inside and outside the cell as well as to their relative permeability across the neuron membrane. Permeability is set by the density, conductance and state (open or closed) of the selective and non-selective ion channels present in the membrane. Active transmembrane ion pumps in neurons and astrocytes maintain the intracellular/extracellular concentration gradients. The three main ions in play are generally potassium $\mathrm{K}^{+}$, sodium $\mathrm{Na}^{+}$and chloride $\mathrm{Cl}^{-}$. The equilibrium (or reversal) potential $V_{\mathrm{R}}$ is determined by the Goldman-Hodgkin-Katz (GHK) equation, which is a generalization of the Nernst equation for multiple ions. The GHK equation for these three ions is:

$$
V_{\mathrm{R}}=\frac{R T}{F} \ln \left(\frac{P_{\mathrm{Na}^{+}}\left[\mathrm{Na}^{+}\right]_{\mathrm{out}}+P_{\mathrm{K}^{+}}\left[\mathrm{K}^{+}\right]_{\mathrm{out}}+P_{\mathrm{Cl}}\left[\mathrm{Cl}^{-}\right]_{\text {in }}}{P_{\mathrm{Na}^{+}}\left[\mathrm{Na}^{+}\right]_{\text {in }}+P_{\mathrm{K}^{+}}\left[\mathrm{K}^{+}\right]_{\text {in }}+P_{\mathrm{Cl}^{2}}\left[\mathrm{Cl}^{-}\right]_{\text {out }}}\right)
$$

where $[\cdot]$ in and $[\cdot]$ out stand for the concentration of each ion inside and outside the cell, respectively. $P_{i}$ is the relative membrane permeability of ion $i$ (in $\mathrm{m} / \mathrm{s}$ ), $\mathrm{R}$ is the ideal gas constant, $F$ is the Faraday constant and $T$ is the temperature. Typical concentrations of these ions are:

$\left[\mathrm{K}^{+}\right]$is higher inside of the neuron $(\approx 140 \mathrm{mM})$ than outside $(\approx 5 \mathrm{mM})$;

$\left[\mathrm{Na}^{+}\right]$is lower inside of the neuron $(\approx 12 \mathrm{mM})$ than outside $(\approx 140 \mathrm{mM})$;

$\left[\mathrm{Cl}^{-}\right]$is lower inside of the neuron $(\approx 10 \mathrm{mM})$ than outside $(\approx 120 \mathrm{mM})$.

Since $\mathrm{Na}^{+}$channels are usually closed at rest, the resting membrane potential is established by the reversal potential of $\mathrm{K}^{+}$and $\mathrm{Cl}^{-}$ions, which are around $-75 \mathrm{mV}$ and -50 $\mathrm{mV}$ respectively. But $\mathrm{Na}^{+}$channels activate sharply as the membrane potential increases towards $-50 \mathrm{mV}$. At that voltage, their openings produce a strong influx of $\mathrm{Na}^{+}$inside the cell, which leads to an increase in the membrane potential. Equilibrium potential of $\mathrm{Na}^{+}$ alone is around $+55 \mathrm{mV}$. The cell membrane never reaches such a high potential since $\mathrm{K}^{+}$and $\mathrm{Cl}^{-}$channels remain open. In addition $\mathrm{Na}^{+}$channels rapidly deactivate and additional voltage dependent $\mathrm{K}^{+}$channels activate at higher voltages. This leads to a rapid decrease of the membrane potential back to its resting value. Such an escape from the usual neuronal resting potential and returning back is called an action potential or spike (Fig. 1.2). It is said that the neuron fired. The spike is usually initiated at the axon initial segment, close to the neuron cell body, and propagated until the end of the axon terminals - the synapses. When a neuron continuously receives excitation from other neurons, it fires repetitively. 


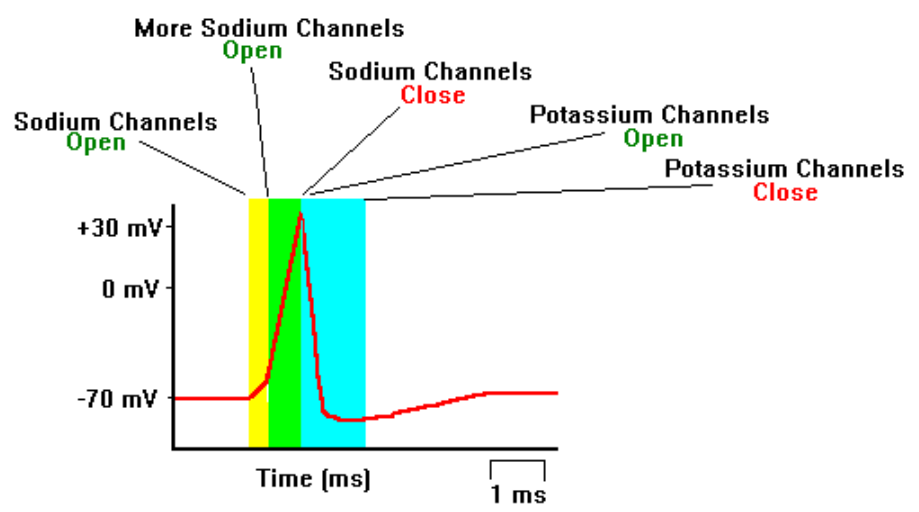

Figure 1.2 Stereotypical action potential

Source: http://faculty.washington.edu

Depending on the exact voltage dependencies and activation and deactivation kinetics of different ion channels present in the neuron membrane, neurons can have very different response properties and excitabilities. One way to characterize the excitability of a neuron is to study how it responds to current injection. For a neuron that can be approximated as a single electrical compartment, the temporal evolution of its potential can be written as:

$$
C V^{\prime}(t)=I_{m}\left(V(t), V_{t}\right)+I_{i n j}(t)
$$

where $C$ stands for the neurons electrical capacitance, $I_{m}$ is the intrinsic membrane current and $I_{i n}(t)$ the injected current. The intrinsic current at a time $t$ depends in general on the membrane potential at time $t, V(t)$ and on the membrane potential history $V_{t}=$ $\left\{V\left(t_{1}\right), t_{1}<t\right\}$. The intrinsic current can be decomposed into different ion currents:

$$
I_{m}=\sum_{k}\left(V-V_{R, k}\right) G_{k}
$$

where $G_{k}$ is the conductance for an ion $k$ and $V_{R, k}$ is the reversal potential for ion $k$. This way of writing the current - as the product of the conductance times the "driving force" $\left(V-V_{R}\right)$ - is a simplification of the Goldman-Hodgkin-Katz flux equation. Here, the conductance $G_{k}$ would depend on voltage history. A now standard way to express the temporal evolution of $G_{k}$ for each current $k$ was introduced by Hodgkin and Huxley in 1952 (Hodgkin and Huxley, 1952), where it is the solution of a set of first order differential equations: 


$$
\begin{aligned}
& G(t)=G_{\max } \varphi(t)^{\alpha} \chi(t)^{\beta} \\
& \dot{\varphi}(t)=\frac{1}{\tau_{\varphi}(V)}\left(\varphi_{\infty}(V)-\varphi(t)\right) \\
& \dot{\chi}(t)=\frac{1}{\tau_{\chi}(V)}\left(\chi_{\infty}(V)-\chi(t)\right)
\end{aligned}
$$

Where $G_{\max }$ is the maximum conductance and $\varphi$ and $\chi$ are channel gating variables. $\varphi_{\infty}(V)$ and $\tau_{\varphi}(V)$ are the steady state value and the time constant of gating for a particular voltage, respectively. Similarly for $\chi_{\infty}(V)$ and $\tau_{\chi}(V)$. The gating rates are usually Boltzmann factors. $\alpha$ and $\beta$ are constants.

Depending on the gating parameters, the equations describing the temporal evolution of all ion channels conductance can give rise to a rich set of dynamic behaviors of the neuron membrane potential (Izhikevich, 2007). Neurons can, however, be classified into three general classes of excitability depending on how they respond to a constant injected current $I_{i n j}$ (Hodgkin, 1948). Class I neurons can fire with an arbitrary small frequency and are also called integrators. Class II neuron have a minimum firing frequency and are also called resonators. And class III neurons fire only once for a constant injected current. These differences in firing behavior can mainly be explained by the non-linear competition of inhibitory (hyperpolarizing) and excitatory (depolarizing) currents with different time courses (Prescott et al., 2008a). Pyramidal neurons in the cortex are mainly class I, although it is argued that it might only be true under in vitro conditions (Prescott et al., 2008b); whereas fast spiking interneurons, which are often inhibitory, are mainly class II. In the auditory pathway, neurons are often class III.

A further complication of neuronal dynamics arises from the neurons geometry, which might makes it inadequate to describe a neuron as a single electrotonic compartment. This is particularly important if different electrical compartments have different ion channel densities, and thus different excitability properties. For example it is thought that the beginning of the axon contains a higher density of $\mathrm{Na}^{+}$channels in comparison to the soma, which makes it more excitable than the soma (Kole et al., 2008; Fleidervish et al., 2010). However, excitation from the synaptic inputs arrives first to the soma. Thus, changes in the distance from the soma to where the spike is initiated can modulate the neurons excitability (Grubb et al., 2011)

Often neurons can be described by much more simple models than the HodgkinHuxley equations. One of the most simple model is the leaky integrate and fire neuron (Lapicque, 1907; Stein, 1967; Knight, 1972; Abbott, 1999; Brunel and van Rossum, 2007). 
This model makes the approximation that the membrane is passive around the neuron resting potential, and that as soon as the voltage reaches a certain threshold, the activation of sodium channels is so sharp that a spike is immediately emitted:

$$
C V^{\prime}(t)=-G\left(V-V_{\mathrm{R}}\right)+I_{i n j}(t)
$$

where $C$ stands for the neurons capacitance, $G$ for its conductance and $V_{\mathrm{R}}$ is the reversal potential, also called leak potential. A spike is initiated when the voltage crosses a certain threshold $V_{T h}$. After a spike, the potential is simply reset to a value close to the resting potential.

By slightly modifying this equation and adding an additional adaptive term that has its own temporal evolution one can obtain the adaptive exponential integrate and fire neuron. With just two first order differential equations and a condition for resetting the membrane potential and the adaptive term after spiking, such a simple model can account for many different neuronal firing behaviors (Naud et al., 2008).

\subsection{Synapses and synaptic transmission}

When an action potential invades a synaptic terminal, the membrane experiences a short depolarization. Voltage-gated $\mathrm{Ca}^{2+}$ channels at the terminal open during the depolarization and $\mathrm{Ca}^{2+}$ enters into the cell during the hyperpolarization phase of the spike. $\mathrm{Ca}^{2+}$ buffers present in the cytoplasm restrict the $\mathrm{Ca}^{2+}$ signal to the proximity of the $\mathrm{Ca}^{2+}$ influx. Several neurotransmitter filled vesicles are positioned at the surface of the plasma membrane in the synapse. SNAREs (Jahn and Scheller, 2006), proteins performing the fusion of the vesicle membrane to the cell membrane, get activated once a $\mathrm{Ca}^{2+}$ sensor (e.g., synaptotagmin) binds to $\mathrm{Ca}^{2+}$ (Südhof, 2004). Before becoming fusion competent, vesicles must undergo a series of steps including docking and priming, which are also $\mathrm{Ca}^{2+}$ dependent (Neher and Sakaba, 2008). The vesicles that can undergo exocytosis immediately upon entry of $\mathrm{Ca}^{2+}$ are part of the so-called readily releasable pool (RRP). Once vesicles are used, new vesicles arrive from a recycling pool (Rizzoli and Betz, 2005). An even larger reserve pool resupplies the recycling pool.

The released neurotransmitter diffuses in the synaptic cleft and reversibly binds to postsynaptic receptors. These receptors can open transiently and elicit a current in the postsynaptic cell. Depending on the neurotransmitter type, the current can be depolarizing (EPSC, excitatory postsynaptic current) or hyperpolarizing (inhibitory: IPSC inhibitory postsynaptic current). The current leads to voltage changes in the postsynapse: EPSP / IPSP (excitatory/inhibitory postsynaptic potential). A miniature EPSC (mEPSC) is the EPSC elicited by the presynaptic release of a single vesicle. 
Several factors can modulate synapse responsiveness and properties. They can be categorized as presynaptic and postsynaptic. Presynaptically: $\mathrm{Ca}^{2+}$ channels can have different kinetics and be in different numbers; $\mathrm{Ca}^{2+}$ buffering can be more or less strong; the $\mathrm{Ca}^{2+}$ sensitivity of the $\mathrm{Ca}^{2+}$ sensor can vary; the size of the RRP and the size and dynamics of the reserve pool can be modulated; the channel-vesicle organization can be different; full vesicle fusion with the cell membrane or kiss-and-run. Postsynaptically: there can be modulations in the receptor type and number; differences in the extrusion of the neurotransmitter from the synaptic cleft can give the neurotransmitter concentration different time courses. Especially receptor kinetics (e.g., binging rates, inactivation, desensitization) can have a strong influence on synapse response. Depending on the particular synapse, it can exhibit a rich set of response properties in response to continuous stimulations. For example, a synapse can show depression or facilitation in its response: a decrease or an increase of the EPSC size with successive stimulations, respectively. RRP depletion can lead to synaptic depression, whereas $\mathrm{Ca}^{2+}$ buffer saturation can lead to synaptic facilitation.

To model the synaptic encoding, one often uses the concept of vesicle pools, transition rates between pools and release probability (Tsodyks and Markram, 1997; Markram et al., 1998; Schneggenburger et al., 2002; Pan and Zucker, 2009). Depending on the level of detail of the model, more or less of the above-mentioned processes can be included.

\subsection{Overview of the peripheral auditory system}

The auditory system bears the task of selecting and converting the signal present in the variations of the sound pressure into relevant information for the brain. Each animal adapted to hear the best in a certain frequency range to better adapt for survival in his own environment. Mammals have wide hearing frequency range, for example in humans it is from $2 \mathrm{~Hz}$ to $20 \mathrm{kHz}$. Animal to animal communication, discrimination of the nature of a sound and its provenance require exquisite temporal analysis and a very sophisticated apparatus.

Detection, processing and analysis of sound by the auditory system occur in several stages. Firstly, a mechanical stage in the outer and middle ear; secondly, a transduction stage from mechanical movement into neural encoding in the inner ear; and finally analysis stages in the brainstem, thalamus and cortex (Fig. 1.3). The sound signal is decomposed in frequencies in the inner ear and for each frequency, temporal changes in intensity and the absolute intensity are encoded. 


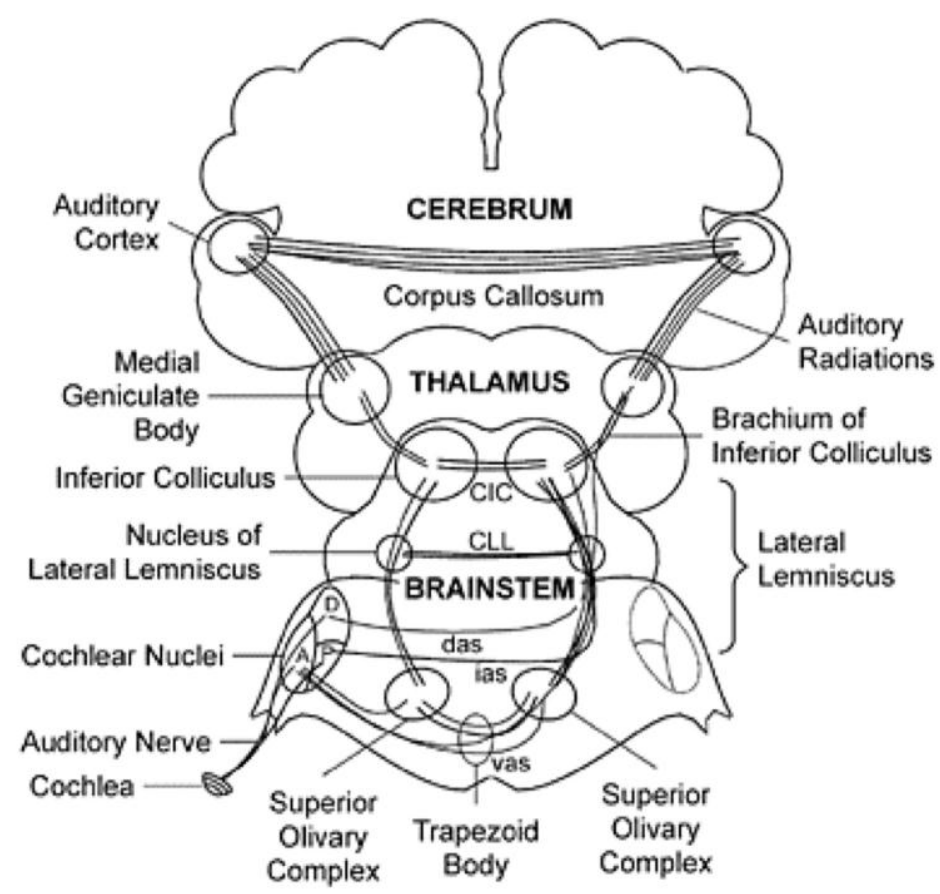

Figure 1.3 Organization of the auditory system

Source: (Gelfand, 2004).

The outer and middle ears transform the air vibrations into mechanical vibrations. Air vibrations are first transformed into vibrations of the tympanic membrane, then of 3 bones, and finally transmitted into fluid and basilar and tectorial membranes vibration in the cochlea, situated in the inner ear (Fig. 1.4). In the cochlea, vibrations are spectrally decomposed by the basilar membrane that has different resonating frequencies along its length. At each frequency, vibrations are non-linearly amplified by outer hair cells (Fettiplace and Hackney, 2006; Ashmore, 2008; Hudspeth, 2008; Peng et al., 2011). Thus, the auditory system performs a Fourier transform of the incoming sound and subsequently analyzes it in parallel frequency channels. The coordinated vibration of the basilar and the tectorial membranes moves the stereocilia of the inner hair cells (IHCs) and produces the opening and closure of mechanotransduction channels. This elicits the entry of ion current, which changes the IHC membrane potential. The IHC voltage thus follows the pressure oscillations (Russell and Sellick, 1978; Dallos, 1985; Kössl and Russell, 1992) up to frequencies inverse to the IHC membrane time constant $(\tau=R C$, where $R$ and $C$ are the resistance and capacitance of the IHC, respectively), which can be as low as $0.1 \mathrm{~ms}$ (Johnson et al., 2011). IHCs exhibit both a AC and a DC component in their voltage responses (Kössl and Russell, 1992). In humans, there are around 3500 
IHCs that are responsible for encoding the whole hearing frequency range in their respective membrane potentials.

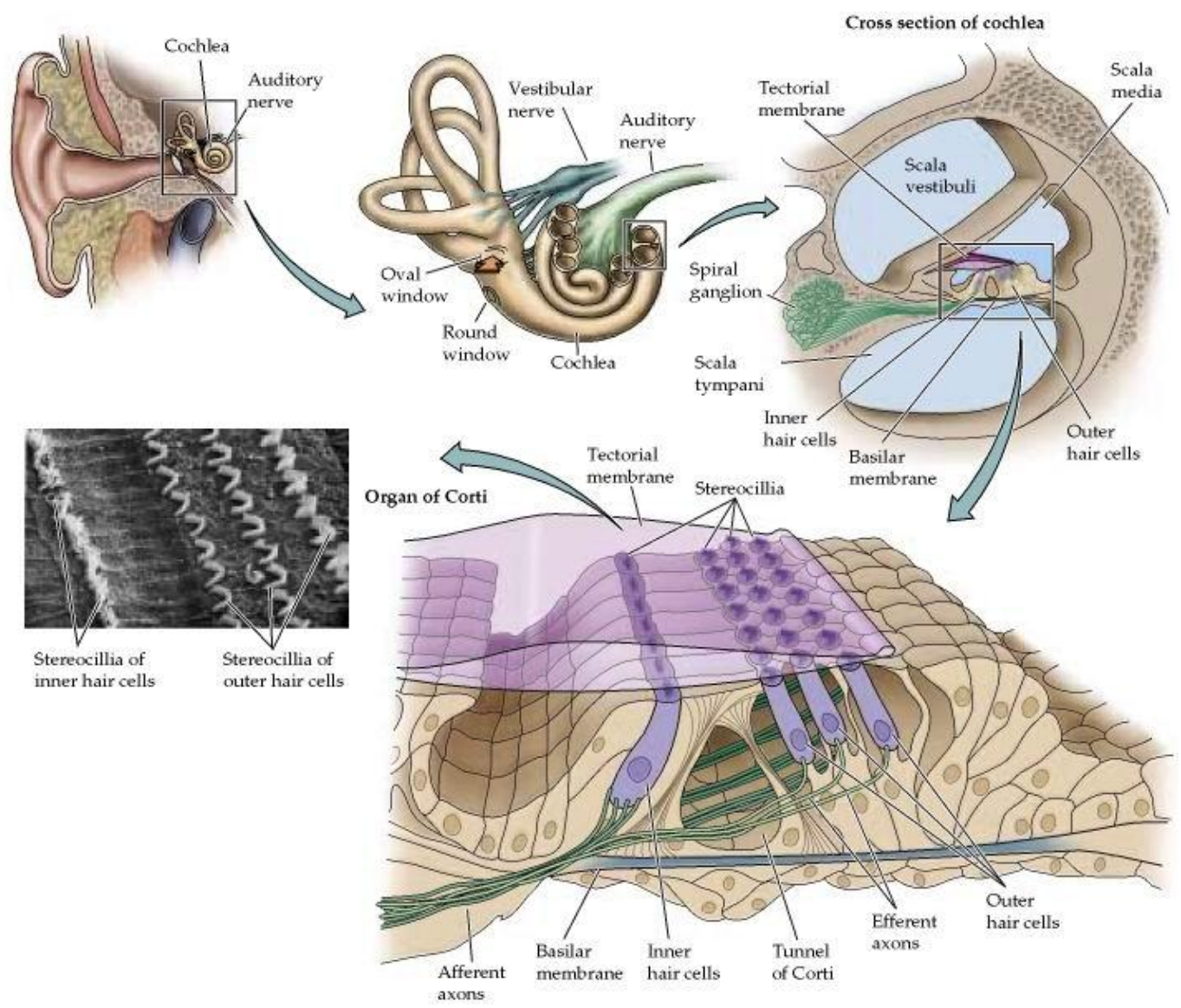

Figure 1.4 Cochlea and organ of Corti in the inner ear

Top left and middle: the cochlea inside the inner ear is a coiled structure, which contains the basilar membrane, which spectrally decomposes sound.

Top right and bottom right: A cross section of the cochlea reveals the presence of one row of inner hair cells $(\mathrm{IHC})$ and 3 rows of outer hair cells $(\mathrm{OHC})$. The OHCs work as mechanical amplifiers by varying their length with changes in their membrane potential (electromotility) and with active hair bundle mechanics. Each $I H C$ encodes the pressure time course at a certain sound frequency in its membrane potential. Each IHC synapses with 5-20 auditory nerve fibers (ANF), which sent sound information encoded in spikes trains to the brain.

Source: (Purves et al., 2001).

Voltage gated $\mathrm{Ca}^{2+}$ channels present at ribbon synapses of the IHCs open and close stochastically in response to changes in the IHC membrane potential. The influx of $\mathrm{Ca}^{2+}$ into synapses triggers the exocytosis of neurotransmitter (glutamate) filled vesicles. Glutamate binds to the AMPA receptors of auditory nerve fibers (ANFs, around 5-20 per IHC) and initiate ANF spiking. At this stage of auditory processing, there is a radical change in sound signal representation: the continuous analog signal of IHCs (their membrane potential) is transcoded into discrete spike timings in ANFs. In humans, there 
are about 30'000 ANFs that encode the entire sound information into trains of action potentials and they form the bulk part of the VIIIth cranial nerve. Due to the tonotopic segregation of IHC and to the fact that each ANF connects to only one IHC, each ANF has a sound frequency at which it responds the best. Thus, the brain can deduce the sound frequency from the set of ANFs that are activated (place code). In addition, since the IHC voltage follows pressure oscillations, ANF's firing rates also exhibits oscillations. It is said that ANF spiking is phase locked to the stimuli. This locking can also be read out by the brain (temporal code) and also contains sound frequency information (Evans, 1978).

The ANFs, also called spiral ganglion neurons (SGNs), have their soma in the spiral ganglion, situated inside the cochlea (Fig. 1.4, lower right). They are bipolar neurons and their axon projects to the cochlear nucleus located in the lower brainstem. Each ANF contacts multiple postsynaptic neurons. Mainly 4 types of cells are present in the cochlear nuclear (Cao and Oertel, 2010): small spherical bushy cells, globular bushy cells, T stellate cells and octopus cells. Each of them receives the input from a different number of ANFs and enhances various features of the sound representation. For example, stellate cells have a wider dynamic range of the stimuli intensity representation than the ANFs; and globular bushy cells have a greater precision during phase locking. Higher stages in the auditory system deal with the further analysis and processing of the auditory information.

Biophysical specializations in synapses and neurons have evolved to meet the high demands on temporal precision in the auditory system. In the next section we will focus on the synaptic transmission from the IHC to the ANFs.

\subsection{Inner hair cell ribbon synapses}

Hair bundle deflections at the top of the IHC induce changes in the IHC membrane potential. The bundles are the "mechanical dendrites" of the IHCs. Just like other sensory cells - for example in the retina - IHCs do not fire action potentials. The resting membrane potential of IHC in vivo is not known with precision but might reside in the range of $-65 \mathrm{mV}$ to $-45 \mathrm{mV}$ (Russell and Kössl, 1991).

The base of the IHC contains synapses that connect to ANFs. They are called ribbon synapses due to the presence of an electron-dense presynaptic body (the synaptic ribbon) that tethers synaptic vesicles (Fig. 1.5) (Matthews and Fuchs, 2010). 

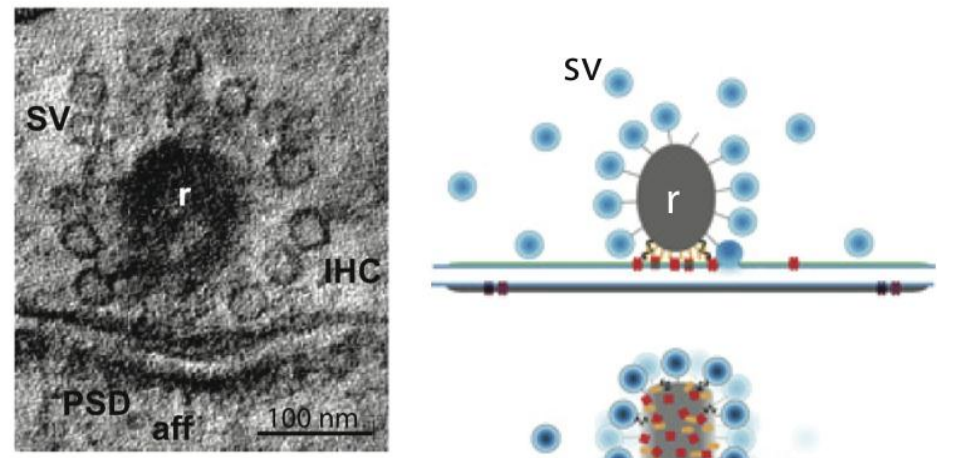

ribbon

ribbon-associated vesicles

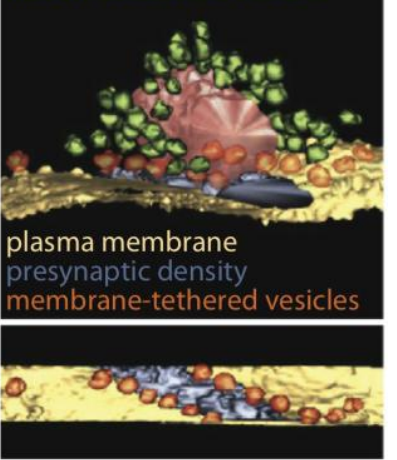

Figure 1.5 The ribbon synapse

Top left: electron micrography of a single thin section. $r$ : synaptic ribbon, SV: synaptic vesicles, PSD: post-synaptic density, aff: afferent fiber ANF. Center left: tomogram-based model of a ribbon synapse.

Bottom left: vesicles at the docked to the plasma membrane. Between the vesicles are position the $\mathrm{Ca}^{2+}$ channels.

Right: scheme of the ribbon synapse with the presynaptic ribbon, vesicles and $\mathrm{Ca}^{2+}$ channels (red).

Adapted from (Frank et al., 2010).

$\mathrm{Ca}^{2+}$ influx that triggers the exocytosis of vesicles originates mainly from $\mathrm{Ca}^{2+}$ channels situated below the ribbon (Roberts et al., 1990; Issa and Hudspeth, 1994; Brandt et al., 2005; Frank et al., 2009; 2010). These are L-type $\mathrm{Ca}^{2+}$ channels (Cav1.3) (Platzer et al., 2000; Brandt et al., 2003). They are rapidly activating and only slightly inactivating, thus enabling sustained $\mathrm{Ca}^{2+}$ influx during prolonged activation. L-type channels exhibit gating modes (Hess et al., 1984): a mode 0 where the channel stays closed all the time, mode 1 where it has short opening and closing, and a mode 2 with prolonged openings. The channel switches stochastically between these modes (Rodríguez-Contreras and Yamoah, 2003) and switching can also be enhanced by drugs like BayK. The channel number per synapse is in the order of 80 (Brandt et al., 2005).

The presence of buffers (Roberts, 1994; Hackney et al., 2005) reduce $\mathrm{Ca}^{2+}$ concentration and shape the exact concentration profile around the ion channel cluster (Neher, 1998a). In particular, due to the close proximity of $\mathrm{Ca}^{2+}$ channels and vesicles, elevated $\mathrm{Ca}^{2+}$ concentrations are exactly at the release sites. $\mathrm{Ca}^{2+}$ sensors present on the vesicles trigger the fusion of vesicles to the plasma membrane (Walter et al., 2011). It is assumed that the binding of 4-5 $\mathrm{Ca}^{2+}$ ions is necessary for the sensor to be activated (Beutner et al., 2001). For the IHC, the nature of the sensor is not clear and one of the 
candidates is otoferlin (Roux et al., 2006). Also the membrane fusion machinery seems to operate without the conventional neuronal SNAREs (Nouvian et al., 2011). A further specialization seems to be that exocytosis occurs in a coordinated fashion: 7 vesicles on average get released synchronously (Glowatzki and Fuchs, 2002; Grant et al., 2010). We will address the mechanisms of synchronous multivesicular release in chapters 4 and 5 .

Several experiments provided evidence that in ribbon synapses, the opening of one or only a few $\mathrm{Ca}^{2+}$ channels suffice to trigger the release of a vesicle (Brandt et al., 2005; Keen and Hudspeth, 2006; Moser et al., 2006; Goutman and Glowatzki, 2007; Jarsky et al., 2010). This is a so called $\mathrm{Ca}^{2+}$ nanodomain control of exocytosis (Neher, 1998b), where the $\mathrm{Ca}^{2+}$ sensor is located very close to the $\mathrm{Ca}^{2+}$ channels (less than 20 $\mathrm{nm}$ ). One of the experimental evidences relies on the fact that only a fast buffer (like BAPTA) can effectively reduce the exocytosis rate at the beginning of a voltage step. A slower buffer (like EGTA), however, cannot because $\mathrm{Ca}^{2+}$ reaches the $\mathrm{Ca}^{2+}$ sensor before the buffer has time to bind.

In comparison, a $\mathrm{Ca}^{2+}$ microdomain control of exocytosis is present when $\mathrm{Ca}^{2+}$ channels are placed further from the vesicle (around $100-200 \mathrm{~nm}$ ) and the opening of several channels is typically required to trigger the release of a vesicle. Microdomain control of neurotransmitter release happens for example at the young calices of Held synapse (Borst and Sakmann, 1996). Having vesicles close to ion channels might seem favorable for precision, as the $\mathrm{Ca}^{2+}$ sensor would feel a very sharp $\mathrm{Ca}^{2+}$ concentration increase upon $\mathrm{Ca}^{2+}$ channel opening. However, vesicle release is then subject to $\mathrm{Ca}^{2+}$ channel stochasticity. Thus, it is not clear whether $\mathrm{Ca}^{2+}$ nanodomain control of exocytosis is an advantage for the system's precision. We will address this question in chapter 6.

Once vesicles are exocytosed, ribbon-tethered vesicles arrive to the plasma membrane (Parsons and Sterling, 2003; Holt et al., 2004; Jackman et al., 2009). The high vesicle exocytosis rates present at ribbon synapses have often been assigned to the presence of the ribbon, that might enable rapid vesicle resupply (Lenzi and Gersdorff, 2001). It is not clear, however, how exactly the ribbon could accelerate RRP replenishment. We will address this question in chapter 3.

Using IHC capacitance recordings (Moser and Beutner, 2000; Schnee et al., 2011), or pre- and postsynaptic recordings (Goutman and Glowatzki, 2007; 2011), it was possible to investigate the dynamics of exocytosis in vitro upon changes in IHC membrane potential. Upon IHC depolarization, the release rate has usually sharply increases and then adapts to a lower steady state value. The first fast component is 
related to the exocytosis of the RRP and is in the order of $10-20 \mathrm{~ms}$. The steady state rate results from a balance between exocytosis and RRP replenishment rates.

The postsynaptic receptor cluster on the ANF is composed of AMPA receptors (Glowatzki and Fuchs, 2002) which bind glutamate. Interestingly, the cluster has a donutlike shape (Meyer et al., 2009). The postsynaptic ANF bouton has a small size (1-2 $\mu \mathrm{m})$ and is thought to reliably encode into trains of spikes the neurotransmitter releases from the IHC (Siegel, 1992; Trussell, 2002). But the degree of this reliability has been questioned (Grant et al., 2010) and will be addressed in chapter 2.

\subsection{Multiquantal release at ribbon synapses}

Ribbon synapses seem to be specialized to exocytose multiple vesicles synchronously (Matthews and Fuchs, 2010). The first evidence for multiquantal release (MQR) arose from the IHC ribbon synapses (Glowatzki and Fuchs, 2002). While patching for the first time the postsynaptic bouton of ANFs, they observed very large EPSCs (up to $800 \mathrm{pA}$ ) and the EPSC amplitude distribution was positively skewed, with a peak at around $50 \mathrm{pA}$ (Fig. 1.6 a-c). In addition, they observed that a large proportion of EPSCs were not monophasic (with a single rise phase and decay), but multiphasic - with multiple peaks. It was interpreted that EPCSs were composed of several (on average 7) vesicles and that the multiphasic EPSCs resulted from the badly synchronized release of multiple vesicles. Furthermore, it was found that the EPSC size distribution was independent of the intracellular buffering conditions and of the membrane potential (Goutman and Glowatzki, 2007). Finally, it was shown that with maturation, the proportion of multiphasic EPSCs to monophasic ESPCs was decreased (Grant et al., 2010) and the EPSC distribution became more symmetric, with the peak at the same amplitude as the mean (350-400 pA).

Other ribbon synapses also exhibit features of MQR. In the bullfrog amphibian papilla afference, it was observed that EPSCs were large (100-200 pA on average), and decreased in size once the hair cell voltage was hyperpolarized (Keen and Hudspeth, 2006; Li et al., 2009) (Fig. 1.6d-e). In rod bipolar cells in the retina, the release of up to two vesicles seemed to be coordinated, and uniquantal release occurred when the synapse was depressed or the voltage was hyperpolarized (Singer et al., 2004; Jarsky et al., 2010) (Fig. 1.6f). For both systems, the rise time of small and large EPSCs was identical (around $0.2 \mathrm{~ms}$ for the frog hair cell and 0.3 for the rod bipolar cell). 


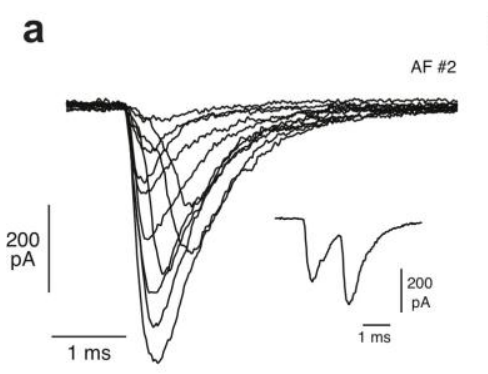

b

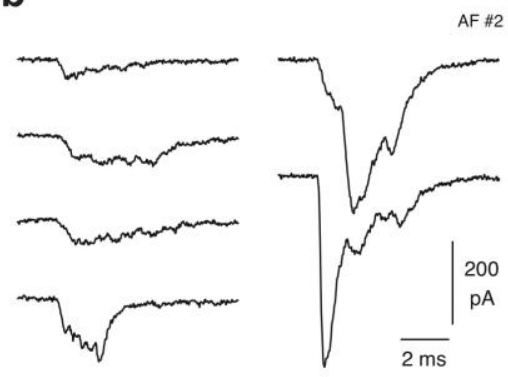

e

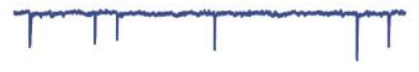

f

C
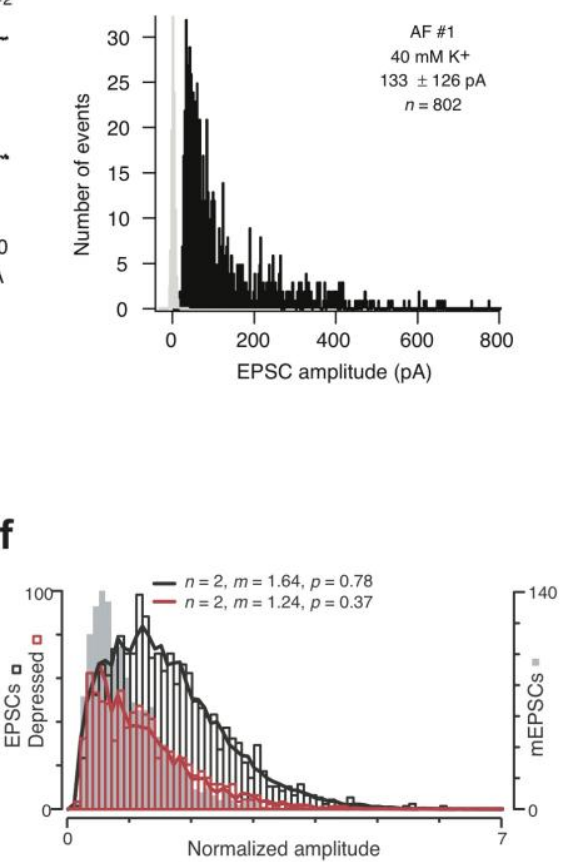

Normalized amplitude
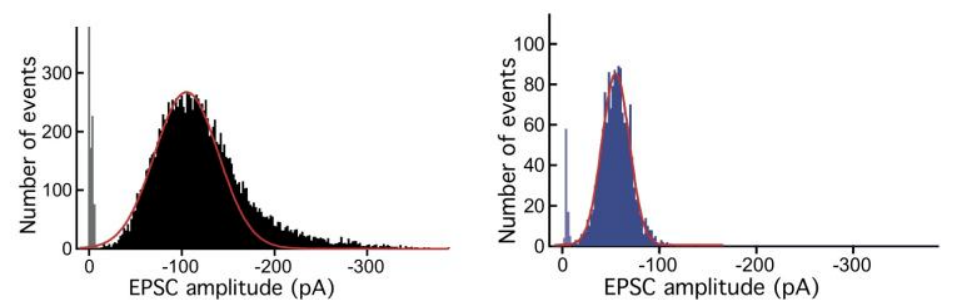

Figure 1.6 Multiquantal release in ribbon synapses

(a-c) from voltage clamp recordings of immature rat inner hair cell afference. Adapted from (Glowatzki and Fuchs, 2002). (a) monophasic EPSCs; (b) multiphasic EPSCs, reminding the superposition of multiple uniquantal EPSCs; (c) EPSC distribution for one fiber.

(d-e) from voltage clamp recording of frog amphibian papilla hair cell afference. Adapted from (Li et al., 2009) (d) the hair cell is unclamped, EPSCs are large; (e) the hair cell is clamped at $-90 \mathrm{mV}$, EPSCs are small.

(f) from the All amacrine postsynapse connected to the rod bipolar cell. Adapted from (Singer et al., 2004). The EPSC amplitude is dependent on the presynaptic holding potential and on whether the synapse is depressed or not.

Proposed mechanisms for MQR (Neef et al., 2007) are synchronization of vesicles by a $\mathrm{Ca}^{2+}$ domain, as during action potentials (Jonas et al., 1993; Wadiche and Jahr, 2001), and compound fusion of vesicles (Pickett and Edwardson, 2006; Matthews and Sterling, 2008; He et al., 2009). But until now the mechanism of MQR remained evasive. In chapters 4 and 5 we will address the question of MQR by modeling the candidate mechanisms.

\subsection{Sound encoding by the auditory nerve fibers}

Action potential firing in the ANFs is driven by neurotransmitter release from IHCs. Usually each ANF is connected to only one presynaptic IHC. The sound frequency at 
which the ANF responds the best is called its characteristic frequency (CF). Since extracellular recordings of action potentials of ANF in vivo are technically less challenging than pre- and postsynaptic patch clamp of IHC and ANF boutons in vitro, there is a significantly richer set of data from the former than the latter. In fact, the responses of ANF in vivo to sound are generally well characterized (Liberman and Kiang, 1978a; Taberner and Liberman, 2005; Avissar et al., 2007; Heil et al., 2011). In addition, several models can successfully reproduce ANF responses to sound (Sumner et al., 2002; Zilany et al., 2009).

Many ANFs fire action potentials in the absence of sound stimuli. Their firing is triggered by neurotransmitter release from the IHC. This "spontaneous" activity can be eliminated by blocking $\mathrm{Ca}^{2+}$ channels in IHCs (Siegel and Relkin, 1987). For a pure tone sound stimulus, ANFs exhibit an adapting response in terms of firing rate. First the rate is transiently very high (up to $1 \mathrm{kHz}$ ), then it decreases to a steady state value (Fig. 1.7a). At the offset of the stimulus, the rate falls below the ANF's spontaneous rate, then recovers. Each ANF has a particular sound pressure level range over which its steady state firing rate changes, called its dynamic range (Fig. 1.7b). Thus, several fibers are required to cover the entire hearing dynamic range in terms of sound intensity. The sound pressure level at which the change of ANF's firing rate becomes noticeable is called the threshold of the fiber. Fibers with high spontaneous rate usually have a low threshold, whereas those with a low spontaneous rate have a high threshold (Liberman, 1978). The mechanisms underlying the ANF heterogeneity and the correlation between spontaneous rate and threshold are not well understood and will be investigated in chapter 6. In particular, it is still an open question whether ANF heterogeneity arise presynaptically or postsynaptically. This question is addressed in chapter 2 . 
a

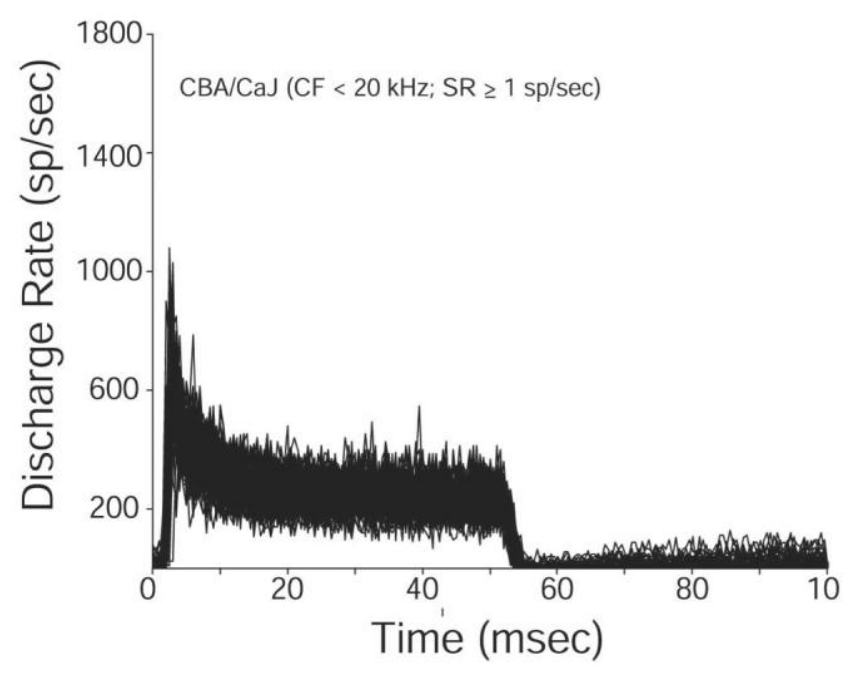

b

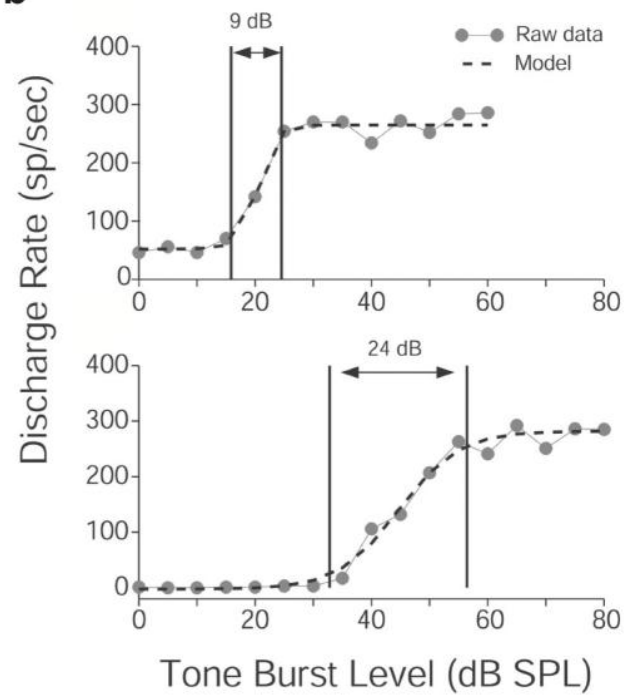

Figure 1.7 Characteristics of ANF responses to tones

(a) superimposed examples of firing rate adaptation in different fibers in response to a sound stimuli $30 \mathrm{~dB}$ above the fibers threshold. Sound stimuli started at $0 \mathrm{~ms}$ and ended at $50 \mathrm{~ms}$. (b) two examples of ANF fibers responses to different sound pressure level. Each fiber has a different spontaneous rate and a different dynamic range. Adapted from (Taberner and Liberman, 2005).

The precision with which ANFs encode sound can be appreciated in their ability to follow pressure variations during a sound tone. The quality of the phase locking is usually measured using the synchronization index (Johnson, 1980), which goes from 0 (no phase locking) to 1 (perfect phase locking without jitter in spike timing during the phase). Phase locking increases with the stimulus intensity (Fig. 1.8a), as oscillations of voltage in the IHC become larger and larger. ANFs with a characteristic frequency above $2 \mathrm{kHz}$ do not phase lock as accurately as for sound frequencies below (Fig. 1.8b). It is not yet understood at which stage of the synaptic transmission this limitation occurs. Possible candidates are the limiting membrane time constant of the IHC, the stochasticity and sluggishness of the $\mathrm{Ca}^{2+}$ channels at the active zone, the slowness of the release machinery, the jitter arising from EPSC variability and finally the jitter in spike initiation. Some aspects of these limitations will be addressed in chapters 2 and 5 . 
a

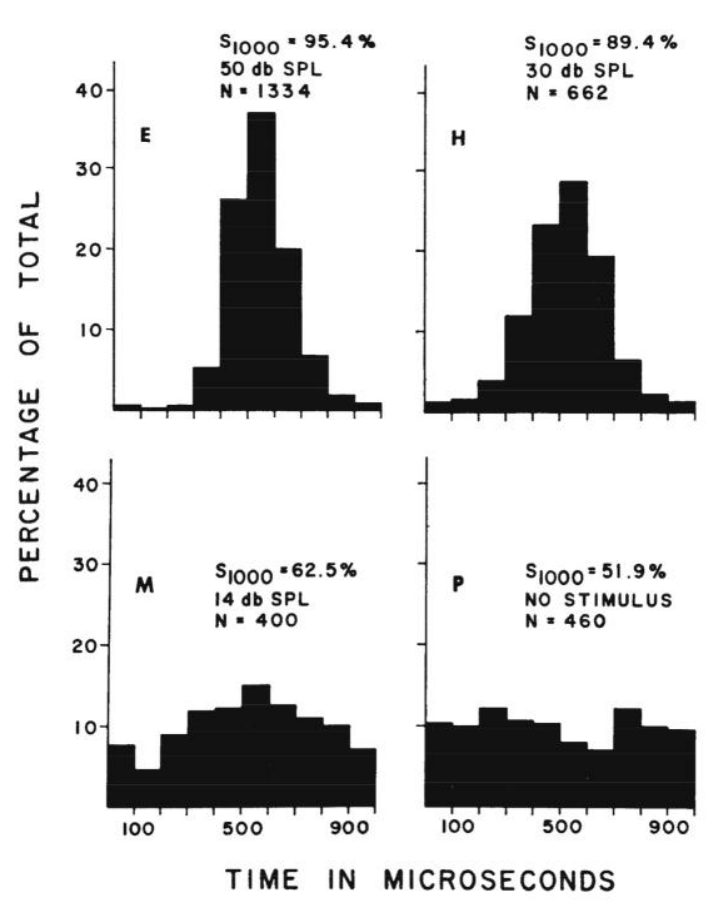

b $\quad 449 \mathrm{~Hz}$
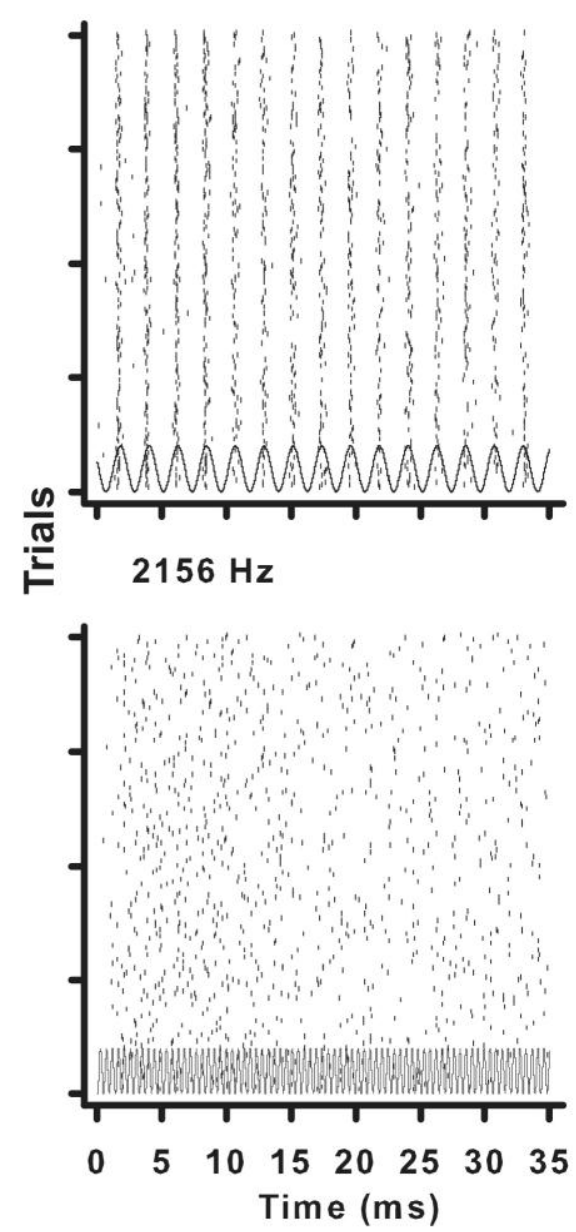

Figure 1.8 Phase locking in ANFs

(a) Increase in phase locking of the ANF response to a $1 \mathrm{kHz}$ tone for increasing sound pressure level (here from $0 \mathrm{~dB}$ SPL to $50 \mathrm{~dB} \mathrm{SPL}$ ). The spontaneous rate of this fiber was around $46 \mathrm{~Hz}$. Adapted from (Rose et al., 1967).

(b) Above $2 \mathrm{kHz}$ frequency tones, ANFs do not follow so well the pressure time course. Each line is a successive trial and dots are spikes in the ANFs. Adapted from (Avissar et al., 2007). 


\subsection{A note on modeling}

The title of the thesis is "Modeling the biophysical mechanisms of sound encoding in inner hair cell ribbon synapses". But modeling is not a goal in itself. Rather: modeling is a tool among many others used to understand biological phenomena. What "to understand" means is a philosophical question. Practically, one of the usual meanings of "understanding" is being able to describe how something works, either phenomenologically, or at a level of detail beyond the one that is already observed. In biology, as in other "reverse engineering" sciences, understanding can also be addressing the questions: "what is something good for?" or "why is something the way it is?". The two mentioned meanings of "understanding" could be related to each other: understanding the role of something at a lower system level, could be almost equivalent to understanding how that something works on a higher system level.

To what reverse engineering is concerned, modeling and theory can provide phenomenological descriptions that enhance our understanding of a system, but without insisting on the meaning of the variables present in the phenomenological description. Modeling can also predict the outcome of several scenarios and mechanisms and see which one is the most suitable to describe observations. But only experiments will be able to confirm or repudiate the predictions a theory make.

For the second meaning of "understanding", by using what might be already known, modeling can make predictions and probe how something could have been. By comparing the system consequences of different scenarios, modeling is able to suggest what something might be good for, what are its advantages, or give a justification of why it is present. In terms of these two types of "understanding", the thesis chapters can be classified as following:

Chapter 2 provides a quantitative description of a neuron. Modeling allowed a concise description and revealed features that were not accessible from direct measurements.

Chapter 3 is of the type "what is it good for". By making assumptions on an existing phenomenon, we tried to reveal what could be its advantages.

Chapter 4 is a "reverse engineering" attempt. By modeling several mechanisms to explain an observation, we tried to find what is more or less plausible.

Chapter 5 uses experiments with the aim to confirm or repudiate the predictions of chapter 4, but these experiments will not be considered as a final "proof" on this matter. Chapter 6 is a mixture of both types of understanding. We addressed how something could work, and what could be its advantages. 


\section{Spike encoding of neurotransmitter release timing by spiral ganglion neurons of the cochlea}

This is a collaborative study with Mark A. Rutherford and Tobias Moser. The paper (Rutherford, Chapochnikov and Moser) related to this study is under review. M.A.R. and I (N.M.C.) contributed equally to this study. M.A.R. performed experiments; I programmed the EPSC-like stimuli and performed modeling. Design, analysis and writing were done in a collaborative fashion.

\section{Short summary}

Information transmission between the inner hair cell and the spiral ganglion neurons (SGNs) has many distinctive features. SGNs fire in vivo with steady state rates of up to $400 \mathrm{~Hz}$ and SGNs can phase lock to sounds of up to 2 $\mathrm{kHz}$ in frequency. In addition SGNs have heterogeneous properties: they have different spontaneous rates, different sensitivities and thresholds to sound pressure level. In this chapter we investigated how the postsynapse, i.e., the SGN contributed to these features and if it was responsible for any. 


\subsection{Abstract}

Precise and reliable action potential generation in type I spiral ganglion neurons (SGNs) is essential to accurately encode the temporal structure of sound. Intriguingly, at the origin of the auditory code in the cochlea, spike triggering relies on the excitatory input from just a single ribbon-type active zone of a presynaptic inner hair cell (IHC). Using whole-cell patch-clamp recordings of SGNs, we characterized action potential generation at the postsynaptic boutons, within the context of synaptic inputs. SGNs were phasic: in response to current injections they generated a single spike with submillisecond latency, responding as high-pass filters. Nearly every neurotransmitter release from the IHC elicited at spike. In voltage clamp, average EPSC size $(\approx 300 \mathrm{pA})$ far exceeded rheobase $(\approx 50 \mathrm{pA})$. Using EPSC-like stimuli, we found that increasing the stimuli amplitude above the physiological range returned less effective latency/jitter reduction than increasing it up to physiological mean. Current integration and spike latencies were well predicted by a two-compartment leaky integrate-and-fire model, including an exponential term to approximate $\mathrm{I}_{\mathrm{Na}}$ voltage-dependence. Only in response to small stimuli was the spike latency sensitive to the SGN baseline potential. The SGN spiking threshold adapted with changes the baseline potential. We suggest that the synapse-spike generator complex of the cochlea is optimized for efficient and temporally precise read-out of IHC neurotransmitter release for generating an accurate spike-time code of sound information. 


\subsection{Introduction}

The auditory system bears one of the most stringent demands on action potential timing precision in the brain (Harnischfeger, 1980; Moiseff and Konishi, 1981; Carr, 1993; Oertel, 1999). For example, the temporal resolution required for binaural sound source localization lies in the order of tens of microseconds (review in (Grothe et al., 2010)). Type I spiral ganglion neurons (SGNs) convey all the auditory information to the brain. Thus, characterizing spike generation in SGNs is crucial to understand the limitations of the original neural code feeding the auditory system. Spike generation in SGNs is governed by release from just a single large active zone (AZ) of IHCs (Perkins and Morest, 1975; Moser et al., 2006). This is in stark contrast to how the signal is further transmitted across subsequent stages: via the largest synapses of the brain - the endbulb and calyx of Held synapses. There, to conserve accurate timing (Gersdorff and Borst, 2002; Yang and Xu-Friedman, 2009), the arrival of the presynaptic spike activates hundreds of small active zones (AZ) (Nicol and Walmsley, 2002; Sätzler et al., 2002). How can the unique ribbon synapse-spike generator complex precisely transmit auditory information?

Inner hair cells (IHCs), the primary sensory receptors of the cochlea, drive 5 to 20 SGNs (Meyer et al., 2009). At each tonotopic location of the cochlea, IHCs transduce the basilar-membrane vibrations into their graded receptor potential (Russell and Kössl, 1992). Synaptic transmission across the IHC ribbon synapse to the SGNs occurs with glutamate release into the synaptic cleft, which is triggered by $\mathrm{Ca}^{2+}$ arrival at the active zone (Sewell, 1984; Beutner et al., 2001; Robertson and Paki, 2002). The binding of the neurotransmitter to glutamate receptors causes the depolarization of the postsynaptic bouton and the short $(\sim 30 \mu \mathrm{m})$ non-myelinated segment. Action potentials are thought to be generated at the heminode adjacent to the foramina nervosa (Hossain et al., 2005). From there the action potential is actively propagated along the myelinated peripheral axon via several nodes of Ranvier, and beyond the loosely myelinated soma along the centrally-projecting axon to the presynaptic terminals of the SGN in the cochlear nucleus. Paradoxically for precise spike timing, the postsynaptic boutons of the IHC afferent synapse display excitatory postsynaptic currents (EPSCs) with very heterogeneous kinetics (Glowatzki and Fuchs, 2002). Moreover, each SGN displays a different distribution of EPSC shapes, which might contribute to heterogeneity among auditory nerve fibers (Grant et al., 2010). However, the impact of this EPSC variability on SGN spike generation is not yet known (Trussell, 2002). What are the attributes of the 
ribbon synapse-spike generator complex that enables rapid, precise and reliable stimulus encoding in the SGNs despite such heterogeneous synaptic input?

Here we performed whole-cell patch-clamp recordings and computational modeling of postsynaptic boutons of type I SGNs to characterize action potential generation driven by synaptic activity or current-clamp stimuli. While nearly every EPSC was sufficient to evoke an action potential, the spike latency and jitter depended upon the stimulus. The average EPSC was much larger than what was required to reach spike threshold. These data show that for sufficiently large EPSC charges, spike timing has little sensitivity to variations in: 1) the mean EPSC amplitude, 2) EPSC kinetics, and 3) the SGN membrane potential. We suggest that the large synaptic conductance, short integration time and phasic firing of the SGN contribute to efficient and temporallyprecise sound encoding.

\begin{tabular}{|c|c|c|c|c|c|c|c|c|c|}
\hline & $\mathrm{R}_{\mathrm{m}}$ & $\mathrm{R}_{\mathrm{a}}$ & $\mathrm{C}_{\mathrm{m}}$ & $V_{\text {rest, }, l=0}$ & $\begin{array}{c}\text { AP } \\
\text { height }\end{array}$ & $\begin{array}{c}\text { AP } \\
\text { width }\end{array}$ & $\begin{array}{c}\text { Onset } \\
\text { pot. } \\
(\mathrm{mV})\end{array}$ & $\begin{array}{l}\text { Thresh. } \\
\text { I }_{\text {inj. }} \\
\text { (pA) }\end{array}$ & $I_{\text {hold }}$ \\
\hline & $(\mathrm{M} \Omega)$ & $(\mathrm{M} \Omega)$ & $(\mathrm{pF})$ & (mV) & $(\mathrm{mV})$ & (ms) & $(\mathrm{mV})$ & $(\mathrm{PA})$ & (pA) \\
\hline ean & 570 & 46 & 3.4 & -72.4 & 61 & 0.79 & -64 & 46 & -19 \\
\hline s.d. & $\begin{array}{r}261 \\
(8)\end{array}$ & $\begin{array}{l}23 \\
(6)\end{array}$ & $\begin{array}{l}1.8 \\
(5)\end{array}$ & $\begin{array}{c}5.5 \\
(11)\end{array}$ & $\begin{array}{l}20 \\
(7)\end{array}$ & $\begin{array}{c}0.12 \\
(7)\end{array}$ & $\begin{array}{c}1.9 \\
(6)\end{array}$ & $\begin{array}{l}11 \\
(8)\end{array}$ & $\begin{array}{c}17 \\
(8)\end{array}$ \\
\hline
\end{tabular}

Table 2.1. Electrophysiological parameters.

Intracellular patch-clamp recordings on boutons of afferent fibers in the cochlear explant. $R_{m}$ is the membrane input resistance measured with $5 \mathrm{mV}$ steps from $-80 \mathrm{mV}$. $R_{\mathrm{a}}$ (access resistance) and $C_{m}$ (membrane capacitance) were estimated with current transients in voltage clamp. $V_{\text {rest }}$ (resting membrane potential) was defined as the mean zero-current potential. Action potential (AP) height, onset potential, threshold current $\left(I_{\text {inj. }}\right)$, and holding current $\left(I_{\text {hold }}\right)$ were measured from a baseline potential of $-802 \mathrm{mV}$ in response to depolarizing stimuli. AP height was determined as the difference between the peak and after-hyperpolarization using near threshold injected current; AP width was measured as the full-width at half-maximum amplitude; onset potential was measured as the point when the spike onset slope reached $30 \mathrm{mV} / \mathrm{ms}$ in response to ramps of depolarizing current. Threshold current was estimated with square pulses at 5-20 pA resolution. Mean and standard deviation (s.d.) values are given for the number of cells $(n)$ yielding reliable measurements.

Analysis: M.A.R. and N.M.C. 


\subsection{Results}

For an initial characterization of action potential generation at the first auditory synapse, we performed whole-cell patch-clamp recordings from the afferent fibers of SGNs, where the postsynaptic boutons contact IHCs in the acutely explanted cochlear apex of rats. In the absence of an experimentally applied stimulus we observed spontaneous action potentials in SGNs (Fig. 2.1A), which are thought to be initiated by $\mathrm{Ca}^{2+}$ dependent synaptic transmission from hair cells both in vitro (rat: (Glowatzki and Fuchs, 2002); frog: (Keen and Hudspeth, 2006; Li et al., 2009); fish: (Trapani and Nicolson, 2011)) and in vivo (cat: (Liberman and Kiang, 1978b; Sewell, 1984)). When action potentials originated from baseline potentials of approximately $-80 \mathrm{mV}$, mean spike heights ranged from 30 to $90 \mathrm{mV}$ between cells, and spike full width at half maximum (FWHM) ranged from 0.42 to $1.5 \mathrm{~ms}$ between cells (Table 2.1).

The distribution of spike heights within an individual cell was unimodal (e.g., Fig. 2.1A,B), with a slight tail at lower amplitudes due to infrequent "second" spikes occurring within $10 \mathrm{~ms}$ after the preceding spike. Varying the baseline potential had a large effect on spike height and peak voltage (Fig. 2.1B, right). From a baseline potential of $-65 \mathrm{mV}$, spikes were vanishingly small and difficult to detect because of the relatively large size, and the rapid onset, of the underlying excitatory postsynaptic potentials (EPSPs). These observations are consistent with steady-state inactivation of voltage-gated $\mathrm{Na}^{+}$channels observed in voltage-clamp recordings of acutely dissociated somata of SGNs, in which a shift from -80 to $-60 \mathrm{mV}$ inactivated $\approx 80 \%$ of the $\mathrm{Na}^{+}$current (Santos-Sacchi, 1993).

\subsubsection{IHC exocytosis evokes SGN spikes with great success}

In auditory nerve fibers, spontaneous spikes result from discrete neurotransmitter release events from IHCs, and it has been proposed that nearly every neurotransmitter release event from the IHC is sufficient to evoke a spike in SGNs in vivo (Siegel, 1992). To assess the efficacy of synaptic transmission for spike generation directly at the synapse, next we quantified the success rate by counting the number of unsuccessful EPSPs and comparing that to the total count of excitatory postsynaptic events (spikes + subthreshold EPSPs; e.g., Fig. 2.1A, see also 2.2A). Also, we compared spike rates in current clamp to the EPSC rates in voltage clamp (e.g., Fig. 2.1C,D) from interleaved recording segments. 
A

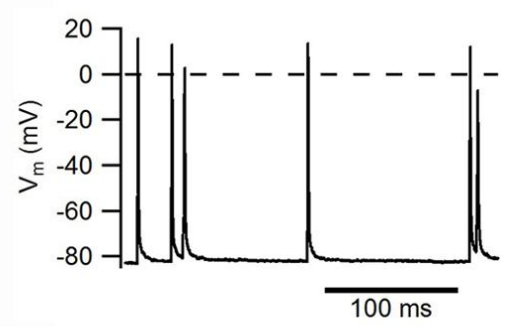

C

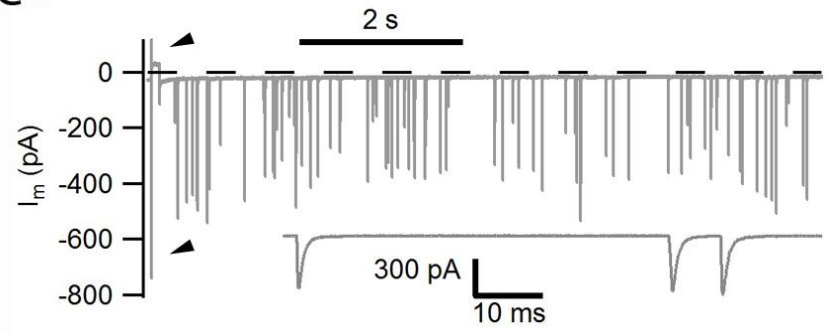

B
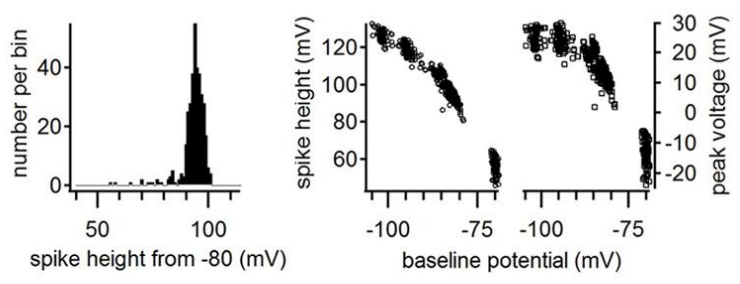

D

Figure 2.1 Nearly every neurotransmitter release event triggered a spike.

A, synaptic transmission from IHCs evoked spikes in the SGN under current clamp. Arrowhead marks a failure of spike generation. P19; $\mathrm{n}=7$ failures, 356 spikes in $60 \mathrm{~s}$; holding current = $-10 \mathrm{pA}$.

B, left: distribution of spike heights, measured from a mean baseline potential of $-83 \mathrm{mV}$. Right: spike height and peak voltage decreased with more depolarized baseline membrane potentials.

C, voltage-clamp recording from the same SGN. Ongoing EPSCs (enlarged in inset) had maximum amplitudes of approximately 500 pA. EPSCs did not evoke action currents, which could be differentiated by their shape and larger amplitude. A brief step to $-65 \mathrm{mV}$ from the holding potential of $-80 \mathrm{mV}$ evoked a stimulus artifact and an action current (arrowheads).

D, interval distributions for action potentials (black) and EPSCs (gray) measured in interleaved current-clamp and voltage-clamp recordings ( $3 \times 20 \mathrm{~s}$ in each mode) demonstrate irregular timing; the distributions are similar and suggestive of a Poisson process. Cumulative probability density functions for spikes and EPSCs were well fit by single exponentials with $\tau=151 \mathrm{~ms}$ and $\tau=182 \mathrm{~ms}$, respectively.

Analysis: M.A.R.

In recordings from three boutons (P17 - P19) which exhibited prolonged, stable, and abundant $(3-7 \mathrm{~Hz})$ spontaneous activity, the inter-event intervals for EPSCs and spikes were Poisson distributed (e.g., Fig. 2.1D), as expected for SGNs after the onset of hearing ?. In one P19 recording, 97\% of EPSPs (1715 of 1767 in 585 s) evoked a spike. In that case the spike rate was $2.9 \mathrm{~Hz}$ and EPSCs occurred at $3.1 \mathrm{~Hz}$ (560 in $181 \mathrm{~s}$ ). In another recording (P17), 81\% of EPSPs (496 of 614 in 200 s) evoked a spike. There the spike rate was $2.5 \mathrm{~Hz}$ and the EPSC rate $3.2 \mathrm{~Hz}(518$ in $160 \mathrm{~s})$. Such low failure rates indicated that most synaptic events were indeed sufficient to trigger an action potential from the zero-current potential. In contrast, two recordings from boutons before the onset of hearing showed lower EPSP rates and generally higher spike-failure rates (see also (Yi et al., 2010)). In one P11 bouton, in total only 50\% of EPSPs evoked a spike. However, over some periods the success rate increased up to $94 \%$, probably reflecting 
periods of enhanced neurotransmitter release due to presynaptic spiking of the immature IHCs (Tritsch et al., 2007).

\subsubsection{Influence of EPSP kinetics on action potential latency}

Neurotransmission events from individual active zones of the presynaptic IHC exhibit significant biological variability (Glowatzki and Fuchs, 2002; Grant et al., 2010; Yi et al., 2010). However, the impact of this heterogeneity on spike generation in the first auditory neuron is unclear. To address this, we assessed the coupling between synaptic input and spike generation using the maximum slope of the spike prepotential (EPSP max slope) as a proxy for the underlying synaptic conductance. Relative to the timing of exocytosis, how long is the latency to spike onset and what is its variance?

We looked at the spikes triggered by EPSPs from IHCs (called "IHC-evoked spikes") and found that the voltage time-courses preceding spikes were variable. First, the EPSP max slopes ranged from 20 to $320 \mathrm{mV} / \mathrm{ms}$ with a somewhat bimodal distribution (Fig. 2.2A). Second, the intervals from EPSP onset to spike onset ranged from 0.3 to 3 ms (Fig. 2.2B), but $>98 \%$ of them ranged from 0.3 to $1.5 \mathrm{~ms}$. Mean latency was approximately $0.6 \mathrm{~ms}$ and the s.d. was $0.3 \mathrm{~ms}$ (CV: coefficient of variation, s.d./mean $\approx$ 0.5). Larger EPSP max slopes were associated with shorter latencies. The scatter plot of latency versus EPSP max slope (Fig. 2.2C) illustrates the strong correlation between the two measures $(r=-0.94$ in the logarithmic scale). Thus, EPSP kinetics had a large effect on the time course of action potential generation in SGNs.

From the scatter plot (Fig. 2.2C), we chose two IHC-evoked spikes as examples of short- and long-latency spikes and, for comparison, we chose two spikes evoked by current-clamp injection (called "CC-evoked spikes") which closely resembled the IHCevoked spikes. Short-latency spikes were preceded by rapid changes in potential followed by a slower depolarization before the spike onset (e.g., Fig. 2.2D, right), whereas longlatency spikes had much smaller EPSP slopes (e.g., Fig. 2.2D, left). Fig. 2.2D, right panel, also demonstrates the effect on spike waveform of a large synaptic conductance versus an extrinsic current (Fatt and Katz, 1951). A convenient way to visualize EPSP and action potential slope as a function of the membrane potential is to look at the phase plots (the time-derivative of $V_{m}$ versus $V_{m}$ ). Fig. 2.2E shows the phase plots for 161 IHC-evoked spikes and the two CC-evoked spikes from panel $\mathbf{D}$. The maximum slopes of the action potentials we observed $(400-500 \mathrm{mV} / \mathrm{ms})$ are comparable to maximum slopes of action potentials measured in pyramidal cell somata, but about one-half as large as action potential slopes measured in the axon initial segment (Kole et al., 2008). 

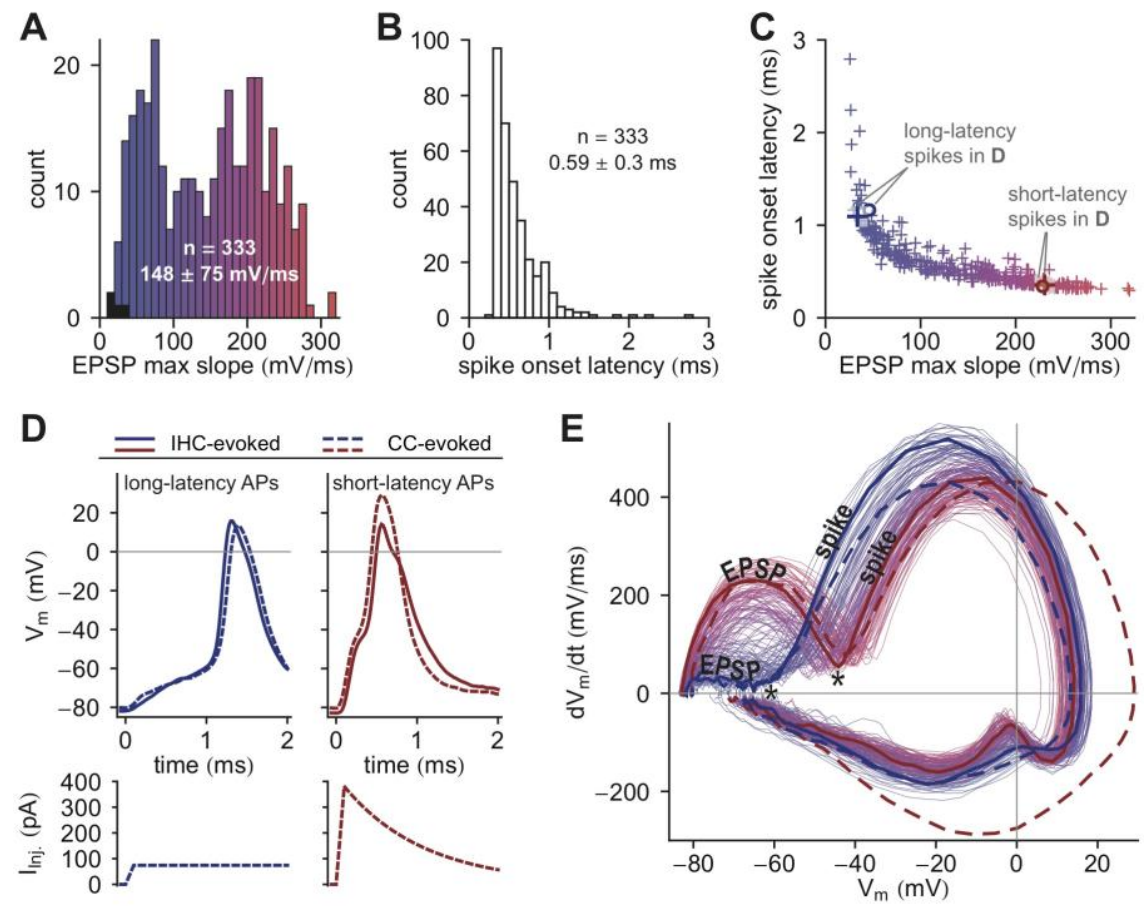

Figure 2.2 Ribbon synapse exocytosis evoked spikes with variable latency.

A, histogram of EPSP max slopes for IHC-evoked spikes (148 $75 \mathrm{mV} / \mathrm{ms}, \mathrm{n}=333$ ). Successful EPSPs are color coded from slowest (blue) to fastest (red). Four slow EPSPs (black) failed to evoke a spike.

B, histogram of spike onset latencies measured from EPSP onset $\left(\begin{array}{lll}0.59 & 0.3 \mathrm{~ms}, \mathrm{n}=333\end{array}\right)$.

C, scatter plot of spike onset latency versus EPSP max slope for IHC-evoked spikes (crosses), color coded as in panel A. The larger crosses correspond to the short-latency (red) and longlatency (blue) IHC-evoked spikes in panel D. Large circles are the CC-evoked spikes in D.

D, comparison of IHC-evoked and CC-evoked spikes. Left: similar spikes with long latencies (1.09 and $1.16 \mathrm{~ms}$ ) and small EPSP max slopes (34 and $46 \mathrm{mV} / \mathrm{ms}$ ). Right: similar spikes with short latencies (0.36 and $0.34 \mathrm{~ms}$ ) and large EPSP max slopes (230 and $228 \mathrm{mV} / \mathrm{ms})$. Lower panels: the stimuli for CC-evoked spikes. Short-latency IHC-evoked spikes were smaller in height and thinner at the peak when overshooting $0 \mathrm{mV}$, consistent with the presence of greater synaptic conductance in comparison to long-latency IHC-evoked spikes.

E, phase plots for IHC-evoked spikes $(n=161)$. Each trace is color-coded according to its EPSP max slope (as in A\&C). Bold traces correspond to the IHC-evoked spikes (solid) and CCevoked spikes (dashed) in panel D. Smaller EPSP max slopes were associated with larger spike slopes and vice versa.

Analysis: N.M.C. 
To summarize so far, with maturation (from P11 to P19) we observed an increase in the reliability of IHC neurotransmitter release to trigger a spike in SGNs, consistent with the previously reported developmental up-regulation of synaptic transmission around hearing onset (Grant et al., 2010). Although nearly every presynaptic release event was sufficient to evoke a spike, spike latency was variable. Because precise spike-timing is important for representation of sound, we sought to better understand the relationship between postsynaptic excitation and spike onset latency. How might the resting conductance and baseline potential affect SGN excitability? What are the SGN firing properties and what is required to trigger a spike?

\subsubsection{Apical SGNs exhibit phasic responses and have a low rheobase}

Auditory nerve fibers are able to fire at hundreds of spikes per second and phase-lock with microsecond precision. Presynaptic mechanisms in the IHC are understood to underlie some response properties of auditory nerve fibers (Moser et al., 2006). Here, to investigate properties intrinsic to the SGN which might support a fast and precise temporal code, we tested the classic excitability of SGNs by injecting square pulses of current into the bouton recording site (Fig. 2.3A-E).

SGNs predominantly fired only one spike at stimulus onset in response to sustained stimuli of any strength or duration. Two of twelve SGNs (P11 and P14) fired a second, smaller spike within a few milliseconds of the first, sub-millisecond latency spike (Fig. 2.3A1,A2). This firing behavior has been termed class III excitability, phasic, or single-spiking (Hodgkin, 1948; Izhikevich, 2007; Prescott et al., 2008a). Here we refer to it as phasic. This single-spiking behavior resembled the phasic or rapidly adapting class of isolated SGN somata (Mo and Davis, 1997a; Mo et al., 2002; Lv et al., 2010) and is in contrast to the slowly adapting class seen in some somatic SGN recordings (Adamson et al., 2002). Hyperpolarizing current pulses evoked inward rectification (Fig. 2.3A1), indicative of the depolarizing current activated by hyperpolarization $\left(\mathrm{I}_{\mathrm{h}}\right)$ which has been studied in SGN somata (Chen, 1997) and in their peripheral non-myelinated neurites in the organ of Corti (Yi et al., 2010). After cessation of strong hyperpolarizing pulses, rebound action potentials were observed (Fig. 2.3A3). 
A1

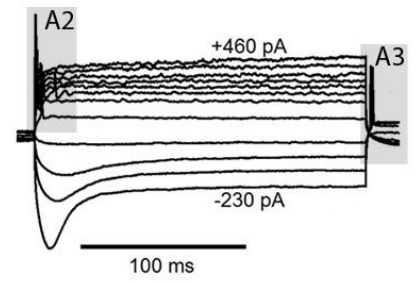

B

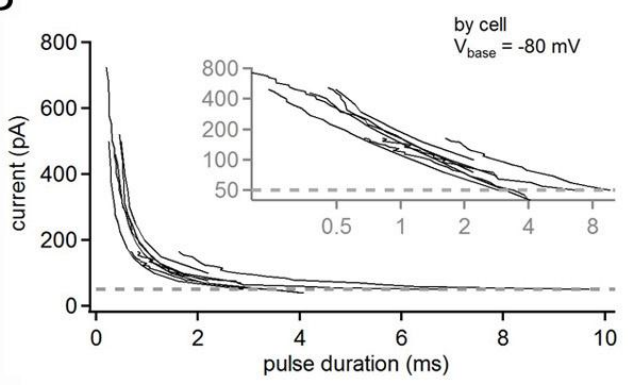

A2

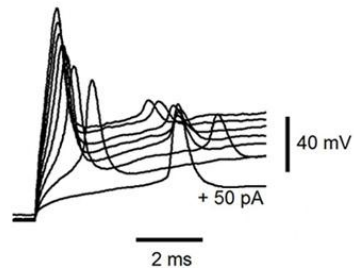

C

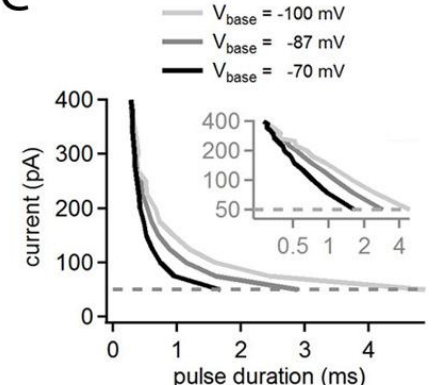

A3

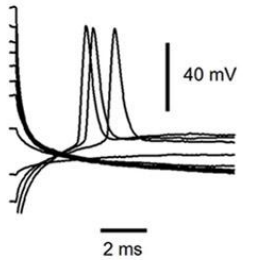

D

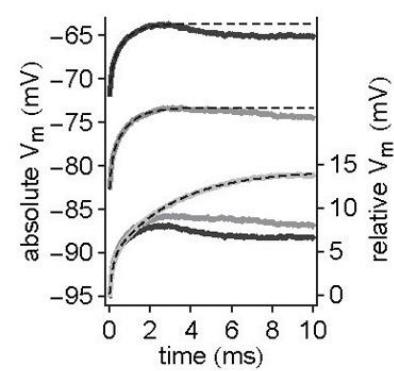

$\mathrm{E}$

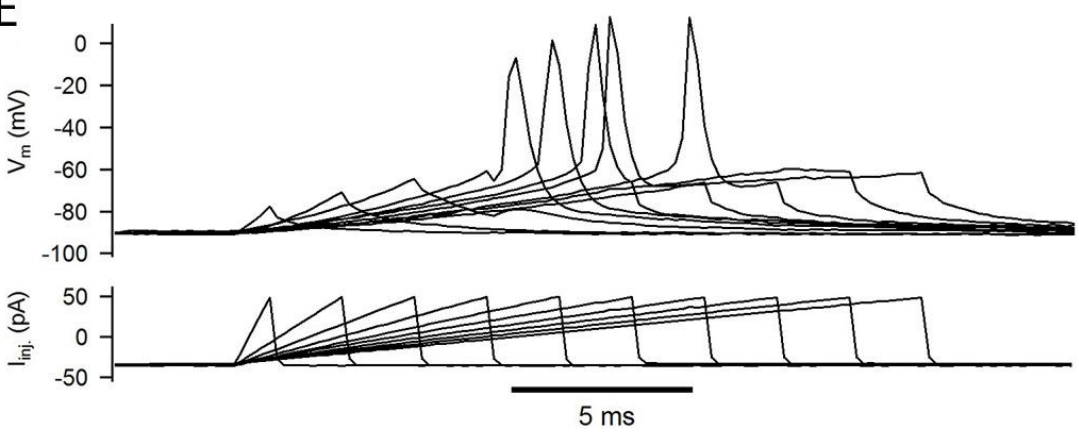

Figure 2.3 Spiral ganglion neurons respond as high-pass filters with low rheobase.

A1, depolarizing current steps evoked one or two spikes at onset (A2). Hyperpolarizing current steps evoked inward rectification, and rebound spikes at stimulus offset (A3).

$B$, strength-duration functions for $8 \mathrm{SGNs}$ stimulated from baseline potentials $\left(\mathrm{V}_{\text {base }}\right)$ of $\approx-80$ $\mathrm{mV}$. Rheobase ranged 35-75 pA. Current pulses were applied at 5-20 pA increments. Dotted lines in C,D show the $50 \mathrm{pA}$ level, insets show double-log scale.

$C$, strength-duration functions for one cell from different $V_{\text {base }}$.

D, averaged subthreshold responses to $20 \mathrm{pA}$ current steps on absolute and relative scale show smaller apparent membrane time constant with depolarization of $V_{\text {base, }}$ due to rapid increase in the membrane conductance. Dashed lines are double-exponential fits up to the response peak: $\tau_{\text {fast }}$ and $\tau_{\text {slow }}$ were 0.09 and $0.74 \mathrm{~ms}$ from $-72 \mathrm{mV}, 0.09$ and $0.81 \mathrm{~ms}$ from $-82 \mathrm{mV}, 0.16$ and $3.0 \mathrm{~ms}$ from $-95 \mathrm{mV}$.

E, lower: ramps from -35 to $+50 \mathrm{pA}$, durations of 1 to $19 \mathrm{~ms}$ in $2 \mathrm{~ms}$ increments. Upper: ramps briefer than $9 \mathrm{~ms}$ or longer than $15 \mathrm{~ms}$ duration failed to generate spikes at these small current levels, demonstrating dependence of spike generation on a minimum charge and rate of depolarization.

Analysis: M.A.R.; N.M.C.: panel D 
One way to characterize and compare neuronal excitability between individual cells is to measure the minimum current amplitudes required to elicit an action potential for different pulse durations, and plot the resulting threshold strength-duration functions (Shepherd et al., 2001). Two characteristics of the strength-duration relationship are rheobase (the current threshold as pulse duration approaches infinity) and chronaxie (the duration required to trigger a spike at a current level twice rheobase). Rheobase ranged from 35 to 75 pA between cells (Fig. 2.3B). Although these rheobase levels we measured were similar to current thresholds determined in SGN somata, the spike latencies we measured were much briefer (e.g., (Mo and Davis, 1997a; Mo et al., 2002)) because less stimulus charge was required to evoke a spike. This observation suggests that spike initiation did not require depolarization of the soma to action potential threshold.

The amplitude of the current step, which evoked the maximum latency, i.e. the minimum current amplitude that was sufficient to trigger a spike in each SGN, is the current threshold or rheobase $\left(46 \pm 11 \mathrm{pA}, \mathrm{n}=8, \mathrm{~V}_{\text {base }}=-80 \pm 2 \mathrm{mV}\right)$. We tested cells over a range of baseline potentials from -100 to $-65 \mathrm{mV}$ by superimposing current steps on steady holding currents. For most cells, $< \pm 30 \mathrm{pA}$ of steady current was enough to offset $\mathrm{V}_{\text {base }}$ over this range. Differences in rheobase were not apparent. However, chronaxie was smaller when $\mathrm{V}_{\text {base }}$ was depolarized because less charge was required to initiate a spike (Fig. 2.3C). Subthreshold voltage responses from more depolarized potentials exhibited rapidly activating $(<2 \mathrm{~ms})$ inhibitory currents, reflected in the decrease of the apparent membrane time constant (Fig. 2.3D). The inhibitory currents were most probably low-voltage activated $\mathrm{K}^{+}$currents (Kv1.1, (Mo et al., 2002); Kv7.4, (Lv et al., 2010)).

Using ramp stimulation we observed a requirement of spike generation for a minimum rate of depolarization (Fig. 2.3E). Although brief current ramps with large slopes and small integrals failed to trigger a spike, ramps that reached the same level with smaller slope delivered more charge before offset and triggered spikes reliably. However, when the ramp's slope was decreased further, it failed to elicit a spike despite its greater charge. The membrane potential began to level-off or decrease before reaching spike threshold, most likely because the hyperpolarizing $\mathrm{K}^{+}$current matched or exceeded the amplitude of the depolarizing current.

Taken together, apical SGNs from mature rats exhibited extremely phasic firing behavior, spiking only once per depolarization and permitting only short latencies. This property might prevent multiple spikes during long EPSCs, and thereby lock spike-times to neurotransmitter release events. SGNs exhibited a threshold not only in terms of 
current amplitude, but also in terms of depolarization rate. This type of excitability is characteristic of class III neurons, which do not respond to slow stimuli and thereby act as high-pass filters (McGinley and Oertel, 2006; Gai et al., 2009). In addition to firing phasically, SGNs also fired with very brief latencies. For current steps of 300 pA, mean latencies ranged from 358 to $648 \mu$ s between cells $(520 \pm 105 \mu \mathrm{s}, \mathrm{n}=5)$.

\subsubsection{Effects of waveform kinetics and size on spike latency and jitter}

Relatively low current threshold (Fig. 2.3) seems to readily explain the high success rate of IHC neurotransmitter release events (Fig. 2.1) for spike generation in SGNs. Although the success rate was high, release events elicited spikes with variable latency (Fig. 2.2), which could affect the reproducibility of spike latencies in an individual SGN and contribute to variance in spike-timing across simultaneously active SGNs. Such variance might influence spike statistics in vivo, for example, first-spike latencies in response to sound onset. Therefore, we further studied the influence of EPSC shape on spike-timing. How much of the variance in spike-timing is due to waveform heterogeneity, and how much is due to jitter inherent to the spike generation process in the SGN?

Within single SGN postsynaptic boutons, EPSCs have substantial variation in total charge, amplitude, rise-time and full width at half maximum FWHM (Glowatzki and Fuchs, 2002; Grant et al., 2010) (see also chapter 5). To determine the jitter inherent to the postsynaptic mechanism of spike generation, we removed waveform variability by injecting repetitions of identical EPSC-like shapes similar to EPSCs recorded in voltage clamp. We then calculated spike jitter as the s.d. of the measured spike onset latencies.

We chose 4 shapes having different kinetics but with equal charge, and scaled their amplitudes over a range of charges. The shapes decreased in speed and current amplitude in ascending order (Fig. 2.4A, lower). Shapes 1 and 2 mimicked "monophasic" EPSCs, having fast rise-times, a very brief plateau, and fast decays. Shapes 3 and 4 had slow rise-times, longer plateaus, and slower decays. Although not multi-peaked, the slow time-course of shapes 3 and 4 were intended to approximate the longer FWHM of "multiphasic" waveforms (Grant et al., 2010). 
A
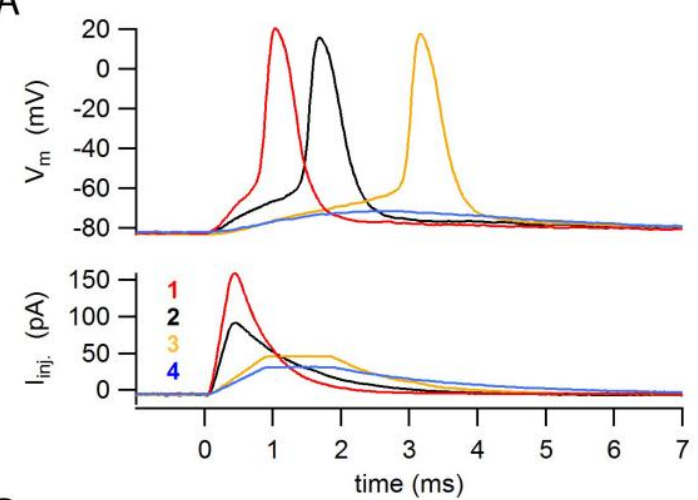

B
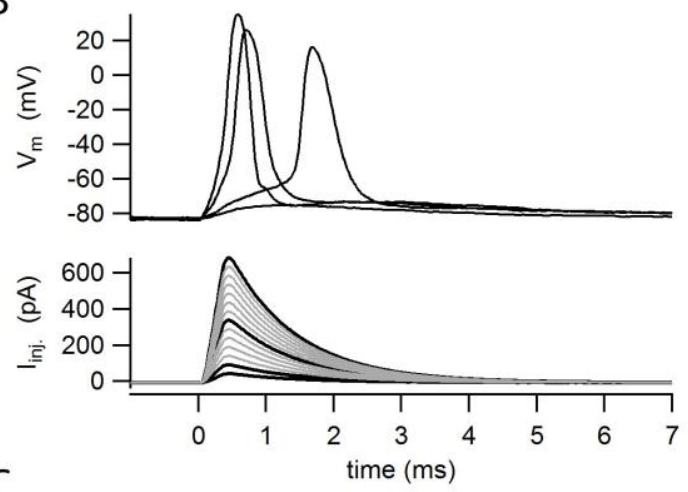

C

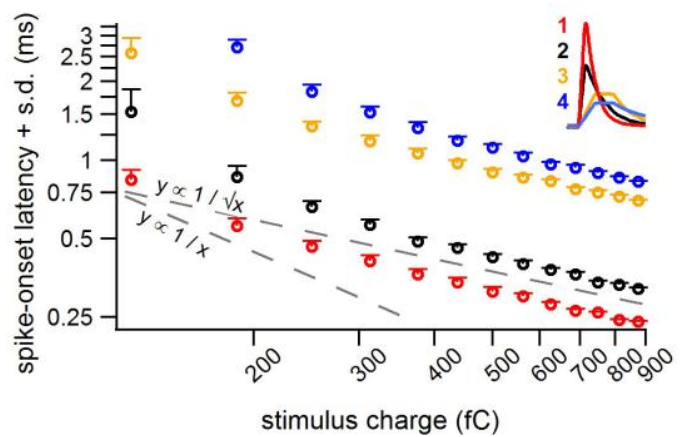

D
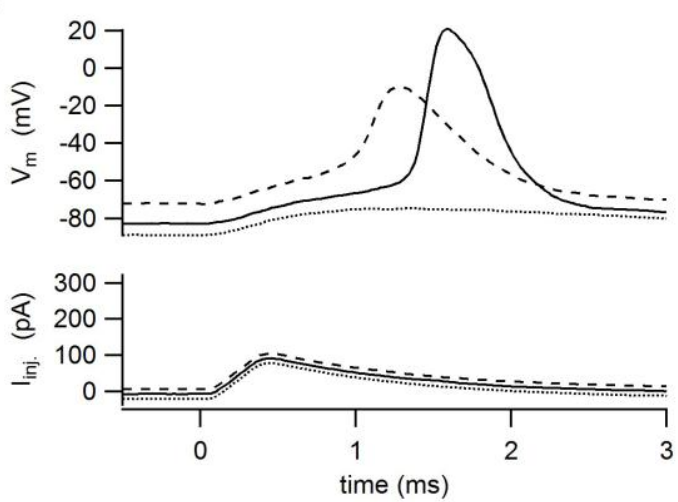

E
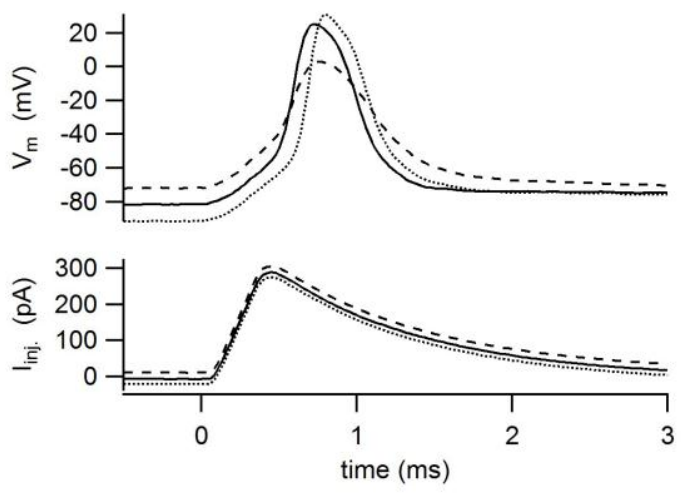

$\mathrm{F}$

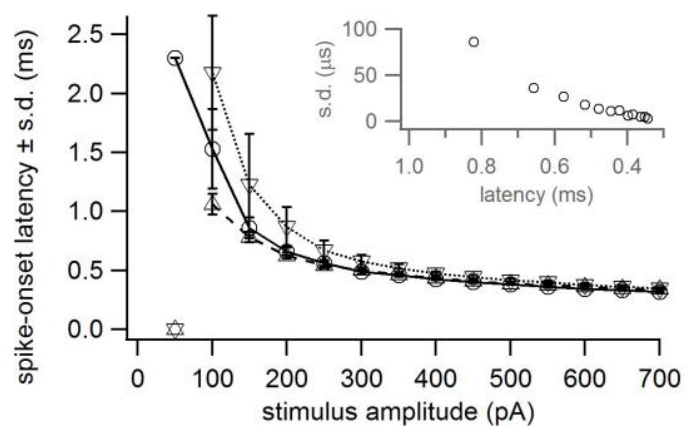

Figure 2.4 Effect of stimulus waveform and SGN baseline potential on spike latency and jitter.

A, four stimulus shapes (lower) were scaled to conserve charge between shapes. Shapes 1 and 2 (red and black) had linear rise-times of $0.3 \mathrm{~ms}$, plateaus of $0.1 \mathrm{~ms}$, and differed only in decay $(\tau$ of 0.5 and $1 \mathrm{~ms}$ ). Shapes 3 and 4 (gold and blue) had linear rise-times of $0.8 \mathrm{~ms}$, plateaus of $1 \mathrm{~ms}$, and differed only in decay ( $\tau$ of 1 and $2 \mathrm{~ms}$ ). In this example, each stimulus delivered only $125 \mathrm{fC}$. Shapes 1-3 evoked spikes with variable latency (upper) but shape 4 failed.

B, each stimulus shape was scaled over a range of amplitudes, which conserved charge between shapes. The smallest amplitudes for each shape (1-4) where 83.3, 50, 26 and 18.4 $\mathrm{pA}$, respectively. Larger amplitudes were integer multiples of the smallest ones. Shown are the range of amplitudes for one series of shape $2(50-700 \mathrm{pA})$. Selected stimulus-response pairs are in bold. Only the $50 \mathrm{pA}$ stimulus failed to evoke a spike.

C, spike latency (+ s.d.) versus charge for each stimulus shape (colored as in A) shows reduction of spike latency and jitter with increasing charge. Each data point is the mean of 5 to 10 repetitions. For small charge the relationship was similar to $1 / x$, but for large charge (> $400 \mathrm{fC}$ ) it was closer to $1 / \operatorname{sgrt}(\mathrm{x})$ (dashed lines), indicating reduced charge-efficiency of spike generation. Note the double-log scale. Waveforms with faster kinetics evoked spikes with shorter latency. 
D, shape 2, amplitude $100 \mathrm{pA}$, was delivered from 3 baseline potentials. Latency depended strongly on baseline potential for such small stimuli.

E, larger amplitude (shape 2, $300 \mathrm{pA}$ ) reduced the shift in spike latency associated with changing the baseline potential.

F, spike latency ( s.d.) versus stimulus amplitude for shape 2 delivered from three baseline potentials $(-94 \mathrm{mV}, \nabla ;-83 \mathrm{mV}, \mathrm{O} ;-72 \mathrm{mV}, \Delta)$. Note reduction of latency, jitter, and sensitivity to baseline potential as stimulus amplitude was increased. Inset: jitter (s.d.) versus mean latency for 5 to 10 repetitions of shape 2 at each amplitude, from $V_{\text {base }}=-83 \mathrm{mV}$. Similar trends were obtained with shapes 1,3 , and 4 .

Analysis: N.M.C.; M.A.R.: plotting

For the smallest charge tested $(31.5 \mathrm{fC})$ all four shapes failed to evoke an action potential. In response to $125 \mathrm{fC}$, shapes 1, 2, and 3 elicited spikes with very different latency (Fig. 2.4A, upper). Fig. 2.4B illustrates a decrease in latency with increasing EPSC size for shape 2. As stimulus size increased, the latencies decreased for all 4 shapes, first rapidly and then more slowly (Fig. 2.4C). For a given charge, the faster waveforms evoked shorter latencies. The longest latencies were approximately $3 \mathrm{~ms}$ in response to near threshold EPSC-like stimulation, and the shortest latencies were around $250 \mu$ s for the largest and fastest shapes tested. Jitter also decreased with increasing size of EPSClike stimuli. For eight repetitions of shape 2 at an amplitude of $300 \mathrm{pA}$, the mean latency was $488 \pm 18 \mu \mathrm{s}(\mathrm{CV} \approx 0.04$ for CC-evoked responses) compared to $590 \pm 300 \mu \mathrm{s}(\mathrm{CV} \approx$ 0.5) for 333 IHC-evoked responses (Fig. 2.2). This confirms that the variance in spike onset latency was dominated by synaptic input, not postsynaptic spike generation. Indeed, the mechanism of spike generation intrinsic to the SGN was precise to within tens of microseconds.

The zero-current potential in whole-cell recordings in vitro may differ from the SGN resting potential in vivo. To assess the influence of SGN baseline potential on spike generation, we applied EPSC-like stimuli from several baseline potentials. Spike onset latency and jitter decreased as the holding potential was depolarized from -92 to $-74 \mathrm{mV}$ (Fig. 2.4F). However, this sensitivity of spike latency to the baseline potential almost vanished for stimulus amplitudes exceeding 300 pA (compare Fig. 2.4D with Fig. 2.4E). Variability or jitter in spike onset latency was relatively large for repetitions of the smallest stimuli, but became vanishingly small (at a resolution of $20 \mu \mathrm{s}$ ) for amplitudes exceeding 200-300 pA.

In summary, even small EPSCs triggered a spike, but EPSC heterogeneity produced variable spike latency and jitter. Increasing the size and/or kinetics of EPSClike stimuli improved the speed and precision of spike-timing. Therefore we expect the developmental up-regulation of EPSC size (287 pA for P19-21 vs. 134 pA for P8-11; (Grant et al., 2010)) and speed (greater proportion of fast monophasic waveforms; 
(Grant et al., 2010)) to reduce the effects of waveform variability and SGN baseline potential on spike latency and jitter. The physiological synaptic input seems appropriately sized for precision and, also, efficiency because increases of stimulus size above the physiological mean $(>\approx 300 \mathrm{pA})$ yielded proportionally less reduction of latency and jitter (Fig. 2.4F).

A

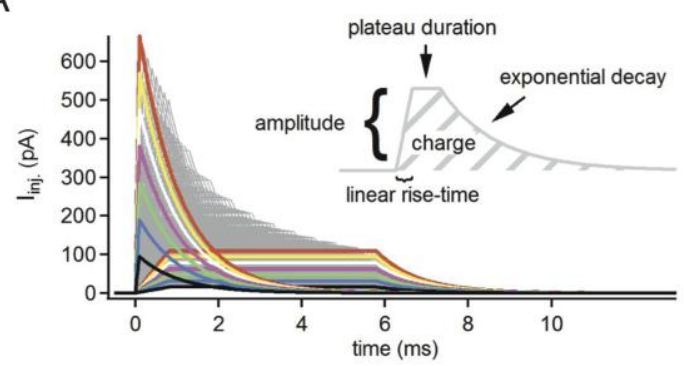

C

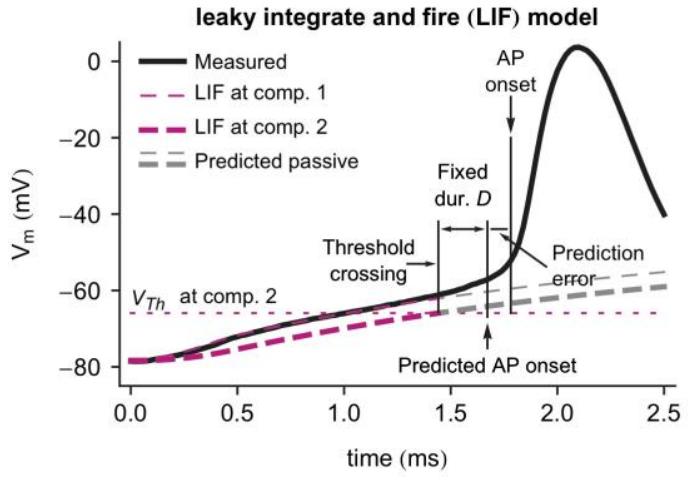

E

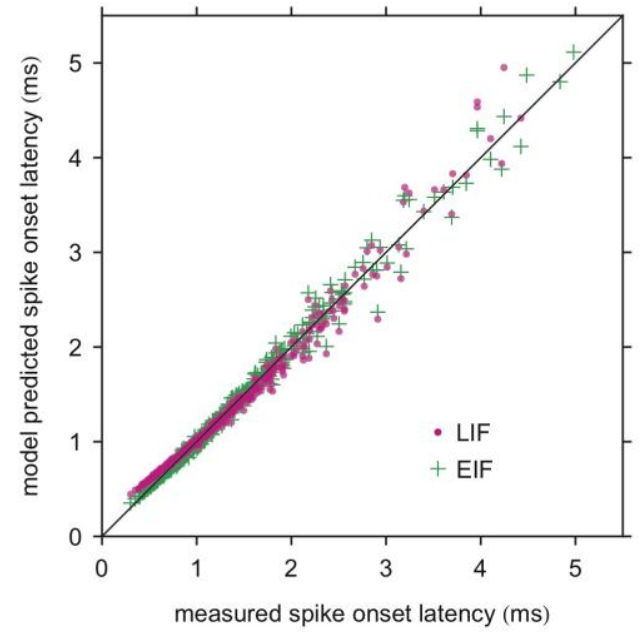

B

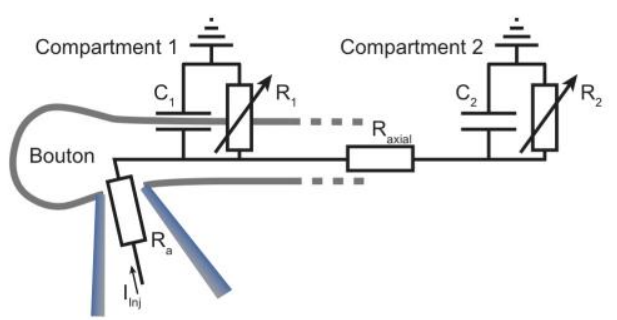

D

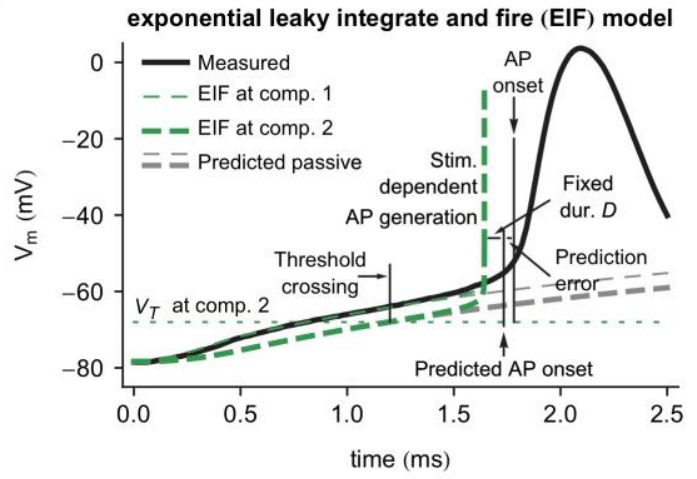

F
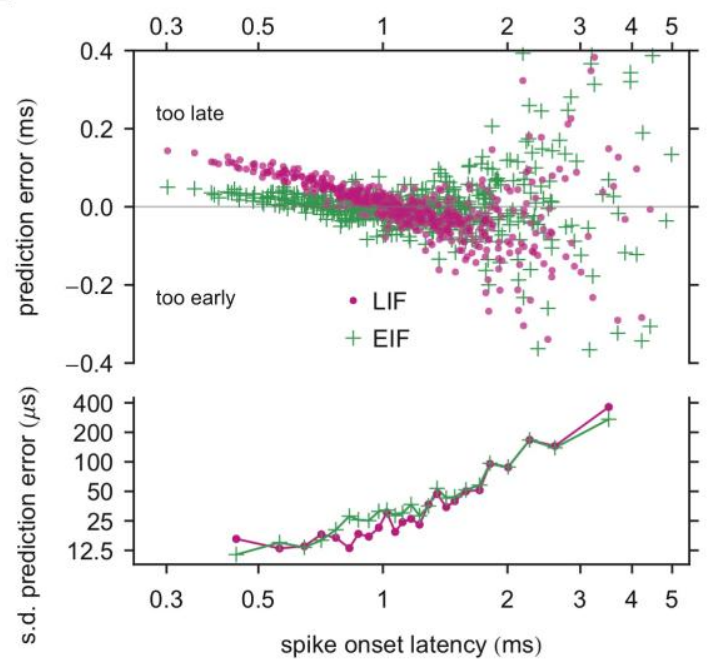

Figure 2.5 Predicting spike latencies with two-compartment LIF and EIF neuron models.

A, hundreds of EPSC-like stimuli (gray) were injected into SGNs (charge from 100 to $700 \mathrm{fC}$ in steps of $100 \mathrm{fC}$, rise time from 0.1 to $0.8 \mathrm{~ms}$ in steps of $0.1 \mathrm{~ms}$, plateau durations from 0 to 5 $\mathrm{ms}$ in steps of $0.5 \mathrm{~ms}$, decay $\tau=1 \mathrm{~ms}$, amplitudes calculated). Bold colored traces show the 
largest- and smallest-amplitude waveforms for each of the 7 charge sets. Inset: characteristics used to define shapes.

B, schematic of the two-compartment circuit. Compartment 1 is connected to the pipette and, via an axial resistance $\left(R_{\text {axial }}\right)$, to compartment 2 .

C, two-compartment leaky integrate and fire (LIF) model prediction and measured response for one stimulus. Shown are the data (black line) and the predicted voltages at both compartments (dashed magenta lines). After the voltage crossed threshold $\left(V_{T h}\right)$ at compartment 2, a spike was predicted to occur at a fixed delay $D$ (fixed for all stimuli). Gray dashed lines show the predicted voltage in both compartments for the case of purely passive membranes. Measured AP onset was defined at $0.15 \mathrm{~ms}$ before the voltage crossed $20 \mathrm{mV}$ below AP-peak. Prediction error $=$ measured - predicted AP onset. The stimulus, a $70 \mathrm{pA}$ plateau with $0.4 \mathrm{~ms}$ linear risetime, started at $0 \mathrm{~ms}$.

D, two-compartment exponential leaky integrate and fire (EIF) model. All same as in C, but here a spike is generated with a fixed delay $D$ after the predicted voltage in compartment 2 (green) diverged toward infinity (see Methods). A long-latency spike is used for the example in C,D for clarity.

E, Model-predicted spike onset latency versus measured spike onset latency for the LIF (magenta) and EIF model (green) demonstrates general accuracy of predictions for latencies from 0.3 - 5 ms.

F, upper: prediction errors versus measured spike onset latency for the LIF model in magenta and EIF model in green (501 responses). Rms latency errors $\delta L$ : LIF $104 \mu \mathrm{s}$, EIF $83 \mu \mathrm{s}$. Coincidence factors F: LIF 98.7\% (8 extra or missing spikes in a total of 600 stimuli with 506 spikes triggered), EIF 98.3\% (10 extra or missing spikes). Lower: s.d. of the prediction error versus measured spike onset latency (calculated using groups of 20 successive points). Model parameters for baseline potential of $-82 \mathrm{mV}$ were - double exponential fit: $\tau_{\text {fast }}=0.07 \mathrm{~ms}, R_{\text {fast }}$ $=40 \mathrm{M} \Omega, \tau_{\text {slow }}=2.3 \mathrm{~ms}, R_{\text {slow }}=450 \mathrm{M} \Omega$. Two-compartment circuit: $R_{1}=1,760 \mathrm{M} \Omega, C_{1}=$ $1.3 \mathrm{pF}, R_{2}=600 \mathrm{M} \Omega, C_{2}=3.8 \mathrm{pF}$, and $R_{\text {axial }}=75 \mathrm{M} \Omega$. LIF: $V_{T h}=-66.5 \mathrm{mV}$, fixed delay $D$ $=0.23 \mathrm{~ms}$. ElF: $V_{T}=-68.6 \mathrm{mV}, \Delta_{T}=1.3 \mathrm{mV}$, fixed delay $D=0.09 \mathrm{~ms}$.

Analysis and modeling: N.M.C.;

panel A: shapes generation: N.M.C.; plotting: M.A.R.

\subsubsection{Modeling the mechanism of spike generation in the SGN}

Unlike a neuron which needs the superposition of many low-amplitude synaptic inputs to initiate an action potential at a relatively distant site, a single IHC active zone drives the SGN bouton and nearby spike generator with high-amplitude input. So far we have shown that the properties of discrete synaptic events have large and immediate effects on spike-timing (Fig. 2.2 and 2.4). To further elucidate the spike generation mechanism, which is specialized for the encoding of sound with accuracy and precision, we combined experiments and modeling (Fig. 2.5 - 2.7). Our goal was to find the simplest analytical neuron model which could predict SGN responses using a minimum number of parameters. SGNs are known to respond at high rates in vivo, with first-spike latencies that vary with sound stimulus parameters (Neubauer and Heil, 2008). To better define the final step in the pathway from sound source to SGN spike, we wanted a model to predict the time-course of spike generation in response to a broad range of individual EPSC-like stimuli (Fig. 2.5).

To construct the neuron model, we first fitted subthreshold voltage responses to depolarizing current steps (e.g., Fig. 2.3E). Because they were better fit by double than 
single exponential functions (mean \pm s.d. for four SGNs: $\tau_{\text {fast }}=0.24 \pm 0.1 \mathrm{~ms}, R_{\text {fast }}=107$ $\pm 13 \mathrm{M} \Omega, \tau_{\text {slow }}=3.3 \pm 0.5 \mathrm{~ms}, R_{\text {slow }}=382 \pm 94 \mathrm{M} \Omega$ ), we used a two-compartment model to predict those responses mathematically (Fig. 2.5B; see Methods), which is the simplest passive electrical circuit able to reproduce them (Pandey and White, 2002). Membrane resistance $(R)$, capacitance $(C)$, and axial resistance $\left(R_{\text {axial }}\right)$ values were obtained for the two compartments in 4 cells: $R_{1}=2.0 \pm 0.6 \mathrm{G} \Omega, C_{1}=1.8 \pm 0.8 \mathrm{pF} ; \mathrm{R}_{2}$ $=485 \pm 149 \mathrm{M} \Omega, C_{2}=7.7 \pm 3.9 \mathrm{pF} ; \mathrm{R}_{\text {axial }}=183 \pm 13 \mathrm{M} \Omega$.

We injected a generalized set of EPSC-like stimuli into SGNs to systematically cover the entire range of physiologically-observed kinetics and amplitudes (Fig. 2.5A). To predict spike onset latencies we considered two simple spike generation mechanisms (see Methods): the leaky integrate-and-fire model (LIF: (Lapicque, 1907; Stein, 1967; Knight, 1972) and the exponential leaky integrate-and-fire model (EIF: (FourcaudTrocmé et al., 2003)). The LIF and EIF neuron models are similar in that they accumulate the stimulus charge on the cell's membrane capacitance and allow charge to escape through the leaky membrane. The models differ only in the spike generation mechanism. For the LIF model (Fig. 2.5C), the voltage follows the predicted passive response and the neuron emits a spike at a fixed delay $D$ after the voltage crosses the fixed threshold $V_{T h}$. For the EIF model (Fig. 2.5D), activation of the spike-generating current is approximated by an exponential function of the difference between the instantaneous voltage $V(t)$ and the fixed threshold voltage $V_{T}$, with a sharpness described by the spike slope factor $\Delta_{T}$. With sufficiently large stimulus current, the membrane potential diverges to infinity in a finite time. The EIF emits a spike at a fixed delay $D$ after the membrane potential reaches $V_{T}+10 \cdot \Delta_{T}$ (i.e., when it is already diverging toward infinity). The supralinear spike-generating current, approximated by the exponential function, endowed the neuron model with an intrinsic mechanism which influenced the time between threshold crossing and spike emission. If EPSP kinetics directly affect the time-course of $\mathrm{I}_{\mathrm{Na}}$ activation, inclusion of this mechanism may thereby reduce the difference between actual spike onset and predicted spike onset.

To test the two spike-generating mechanisms, we added the LIF or EIF mechanism to cellular compartment 2 , or to both compartments. We determined the optimum parameters ( $V_{T b}$ for the LIF; $V_{T}$ and $\Delta_{T}$ for the EIF) by minimizing the error between model predictions and electrophysiological data. Goodness of fit was assessed in terms of latency error $\delta L$ and the spike coincidence factor $F$ (Methods). We found better latency predictions for both models when we placed the spike generator in the second 
compartment (LIF: $26 \pm 17 \%$ smaller $\delta$ L, $\mathrm{p}=0.002, \mathrm{n}=6$; EIF: $35 \pm 23 \%$ smaller $\delta L$, $\mathrm{p}=0.03, \mathrm{n}=4)$. Both models predicted the data with high accuracy. For the EIF, $\delta L$ was $77 \pm 25 \mu$ s and $F$ was $99.0 \pm 0.8 \%, \mathrm{n}=4$. The LIF predicted latencies with a somewhat larger error $(87 \pm 76 \%$ larger $\delta L$ on average in comparison to the EIF, $\mathrm{p}=$ $0.05, \mathrm{n}=4) . V_{T}$ of the EIF was not significantly different than $V_{T b}$ of the LIF $\left(V_{T b}-V_{T}\right.$ $=0.7 \pm 1.2 \mathrm{mV}, \mathrm{p}=0.15, \mathrm{n}=4)$. The spike slope factor $\Delta_{T}$ was $1.4 \pm 0.5(\mathrm{n}=4)$.

Fig. 2.5E exhibits the model predictions versus the measured latencies for a representative SGN stimulated with hundreds of different EPSC-like shapes, for one presentation of each shape. The relatively small error of the model predictions for single instances of each stimulus demonstrates both the accuracy of the model and the relatively deterministic nature of the SGN response. The prediction error of both models was very small for latencies $\leq 1 \mathrm{~ms}$. Only for latencies above $\approx 1.8 \mathrm{~ms}$ did the errors drastically increase (Fig. 2.5E). The increase in prediction error for long latencies may be mainly explained by variability intrinsic to the experiment (see Fig. 2.4F, inset: increase in jitter, s.d., for long latencies).

In summary, although SGNs are phasic and therefore not entirely described as simple "integrators," their first-spike latency in response to a wide range of EPSC-like shapes could nonetheless be accurately predicted by simple leaky integrate-and-fire models. The EIF had less systematic error than the LIF, as it incorporated a stimulusdependent effect on $\mathrm{I}_{\mathrm{Na}}$ activation around spike threshold. The spike generator was better placed in compartment 2 , indicating that the spike generator is not directly at the bouton. However, the relatively small capacitance of compartment $1(\approx 1.8 \mathrm{pF})$, suggests that the spike generator is very close to the synapse. The small value of $\Delta_{T}$ for the EIF and the good performance of the LIF indicate a sharp and rapid spike initiation, important for speed and precision of SGN responses. Such simple models provided an easy way to predict the spike latencies for discrete EPSCs, however, more physiological models will be required (Herz et al., 2006) to predict the neuron's responses to high rates of EPSCs. 
Data:

- spike latency (ms); $\times$ no spike model : $\quad$..... spike latency (ms); $X$ no spike
Data:

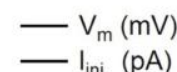

Model (mV):

compartment 1, passive

- compartment 2, ELIF

........ threshold, ELIF
A

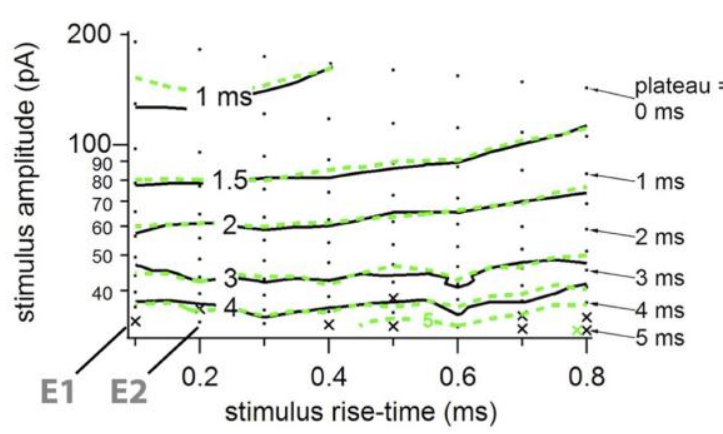

B

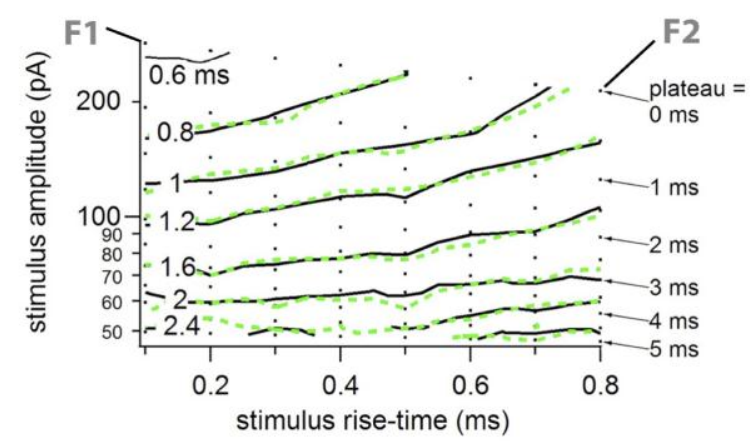

C

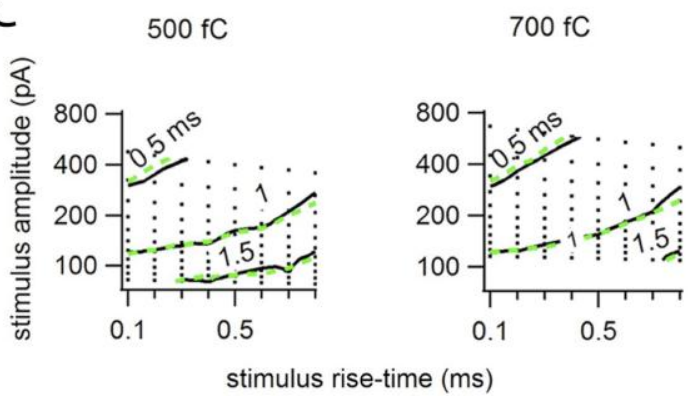

D1

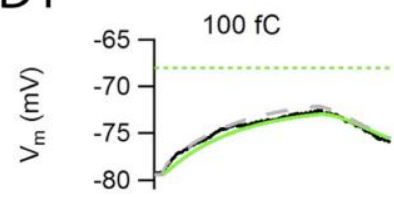

$\mathrm{I}_{\text {inj. }}$

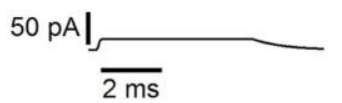

E1

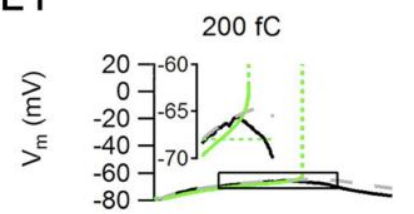

I inj. $100 \mathrm{pA}$ |
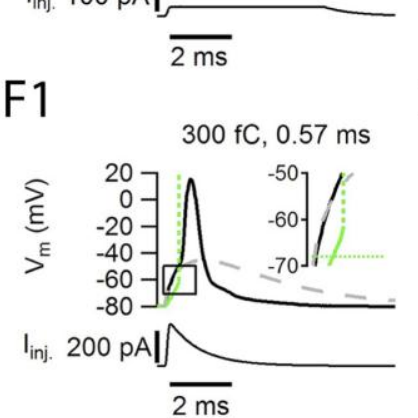

F2

D2

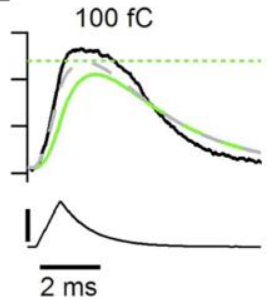

E2

$200 \mathrm{fC}, 4.8 \mathrm{~ms}$
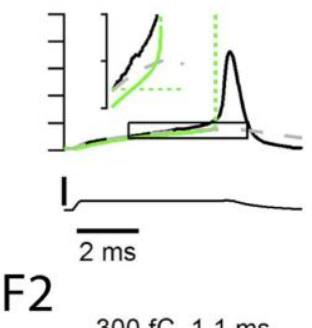

$300 \mathrm{fC}, 1.1 \mathrm{~ms}$

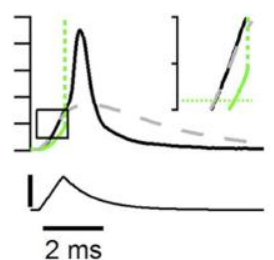

G

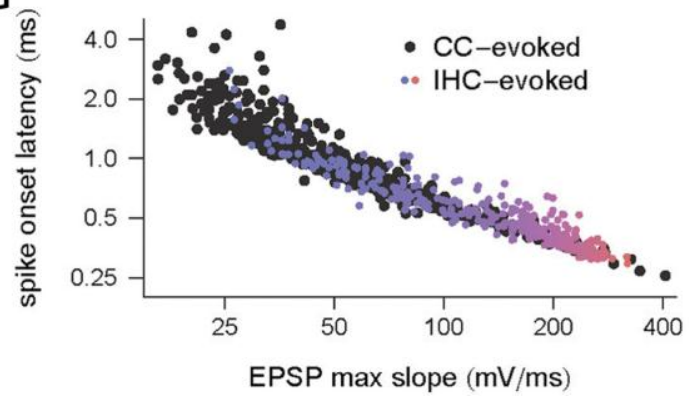

Figure 2.6 Effect of waveform kinetics on latency: data and model predictions.

A, Latency contours in $200 \mathrm{fC}$ parameter space. The black points on the graph represents 88 stimuli of variable amplitude (31-190 pA, y-axis), rise-time (0.1-0.8 ms, $x$-axis), and plateau (0$5 \mathrm{~ms}$, iso-plateau bands labeled on right), each with a total charge of $200 \mathrm{fC}$. Stimuli evoked spikes for all but the smallest waveforms (black $X=$ failure; $n=9$ ). Spike onset latencies were plotted as solid black contour lines ( 1 to $4 \mathrm{~ms}$, labeled in black). Spike onset latencies for the EIF model are overlaid as green dashed contour lines (green $X=$ predicted failure, $n=1$ ).

B, in the $300 \mathrm{fC}$ parameter space, every stimulus evoked a spike. Latencies (black contour lines) were accurately predicted by the EIF model (green dashed contours).

C, spike latency contours for the 500 and $700 \mathrm{fC}$ parameter spaces illustrate reduction of spike latency for larger stimuli, however, reduction in spike latency was reticent when stimuli were increased above $400 \mathrm{fC}$.

D, Stimulus-response pairs for two sub-threshold stimuli $(100 \mathrm{fC})$. The stimulus $\left(\mathrm{I}_{\text {inj., }}\right.$ lower part of each panel) and the cell's response $\left(\mathrm{V}_{\mathrm{m}}\right)$ are shown in solid black. The passive response of 


\begin{abstract}
the model circuit in compartment 1 is shown as a dashed gray line. The response of the EIF model is shown as a solid green line. Dotted green line is the EIF model threshold of -67.5 $\mathrm{mV}$. D1: the data, the passive response, and the EIF model overlaid precisely. D2: some nearthreshold behavior was not predicted by the model.

E, two stimulus-response pairs (as in panel D) from the $200 \mathrm{fC}$ parameter space, labeled in panel A. Box shows area enlarged in inset. E1: a failure of spike generation where the model predicted a spike. E2: similar near-threshold stimulus triggered a long-latency spike.

F, two stimulus-response pairs from the $300 \mathrm{fC}$ parameter space, labeled in panel B. Each inset enlarges the area around spike threshold, where the response of the SGN and the EIF model deviated from the passive response of the two-compartment circuit. After crossing threshold, the model predicted that spike onset would occur in $0.09 \mathrm{~ms}$ from when the function diverged toward infinity (dotted green vertical lines in panels $\mathrm{E}$ and $\mathrm{F}$ ).

G, comparing spike onset latency as a function of EPSP max slope for CC-evoked spikes (black) and IHC-evoked spikes (blue to red, re-plotted from Fig. 3C) revealed a very similar relationship.

Analysis and modeling: N.M.C.; M.A.R.: plotting all panels but G.
\end{abstract}

\title{
2.2.6 EPSC-like stimulation and comparison to synaptically-evoked spikes
}

Browsing through the stimulus parameter space, we compared spike onset latency in the experimental data and the two-compartment EIF model in response to individual EPSClike stimuli such as in Fig. 2.5A. Fig. 2.6A-C illustrates measured and predicted spike onset latencies as contour plots through stimulus parameter space for each charge set. The accuracy of the predicted latencies to the data can be appreciated by the overlap of the green contour lines (model) with the black contour lines (data). Contour lines show the range of stimuli, which evoked spikes with equal latency. When holding charge constant, spike latency was more sensitive to changes in amplitude than in rise-time.

With a total charge of $200 \mathrm{fC}$, only the slowest and smallest stimulus shapes failed to evoke a spike from a holding potential around $-80 \mathrm{mV}$ (Fig. 2.6A). The fastest and largest 200 fC stimuli (150 - 200 pA, 0.1 - 0.4 ms rise-time) evoked spike onset latencies $<1 \mathrm{~ms}$, while the slowest successful waveforms evoked latencies $\approx 4.5 \mathrm{~ms}$. Most physiological EPSCs have charges of 150 - $350 \mathrm{fC}$. When comparing the ranges of latencies for different charge sets within cells, we observed relatively large latency reduction when increasing from the $200 \mathrm{fC}$ to the $300 \mathrm{fC}$ parameter space (e.g., Fig. 2.6A,B). In comparison, we observed less latency reduction when increasing to larger charge sets (Fig. 2.6C).

Traces of experimental stimulus-response pairs and model predictions are shown in Fig. 2.6D-F. Fig. 2.6D shows two subthreshold stimulus-response pairs and model predictions from the $100 \mathrm{fC}$ parameter space. For $100 \mathrm{fC}$ stimuli, only two and six stimuli evoked a spike, respectively, in two cells tested. They were not predicted by the model. Fig. 2.6E shows two stimulus-response pairs and model predictions from the $200 \mathrm{fC}$ parameter space. In rare cases the model predicted a spike when none occurred (Fig. 
2.6E, left): this happened in the parameter region where the neuron's responses were less deterministic (e.g., 4.5 and $5 \mathrm{~ms}$ plateau in Fig. 2.6A). Fig. 2.6F shows two stimulusresponse pairs from the $300 \mathrm{fC}$ parameter space, with the EIF model prediction in compartment 2 and the predicted passive response of the cell in compartment one. As the membrane potential crossed threshold, the recorded data and the EIF model began to deviate from the passive response.

To compare our EPSC-like stimulation with synaptic conductance excitation, we plotted the spike onset latency versus EPSP max slope for the CC-evoked and the IHCevoked spikes (from Fig. 2.2C). The overlap between the CC-evoked and IHC-evoked data sets (Fig. 2.6G) confirmed that a range of our EPSC-like shapes were good approximations of synaptic excitation for the study of first-spike latency in SGNs. This allows one to deduce the EPSC-like stimuli that produced similar pre-potentials and spike onset latencies as did physiological EPSPs (i.e., those shapes eliciting latencies $<1.5 \mathrm{~ms}$ ).

Finally, to complement the study of spike latency, we investigated the sensitivity of spike onset potential and threshold potential to changes in $V_{\text {base }}$ (Fig. 2.7A,B). Spike onset potential is typically defined as the voltage where the rate of depolarization exceeds a particular value, here $30 \mathrm{mV} / \mathrm{ms}$ (i.e., the sudden slope change or visible "kink" in the spike waveform, sometimes called action potential threshold). Threshold potential, in contrast, is the voltage required to result in the initiation of an all-or-none action potential. Here, threshold potential was defined as the optimum $V_{T h}$ from the twocompartment LIF model. Since the voltage evolution depended upon the solution for the two-compartment circuit (see Methods), the estimated voltage thresholds $V_{\text {Th }}$ ranged over 2 to $6 \mathrm{mV}$ for each combination of cell and baseline potential we tested (Fig. 2.7C). We found that both the spike onset potential and absolute threshold shifted to more depolarized potentials with depolarization of $\mathrm{V}_{\text {base. The }}$ This theshold shift partially compensated for shifts of $V_{\text {base}}$, resulting in comparatively small shifts in relative threshold, which should decrease the sensitivity of spike latency to changes in $\mathrm{V}_{\text {base. }}$. 

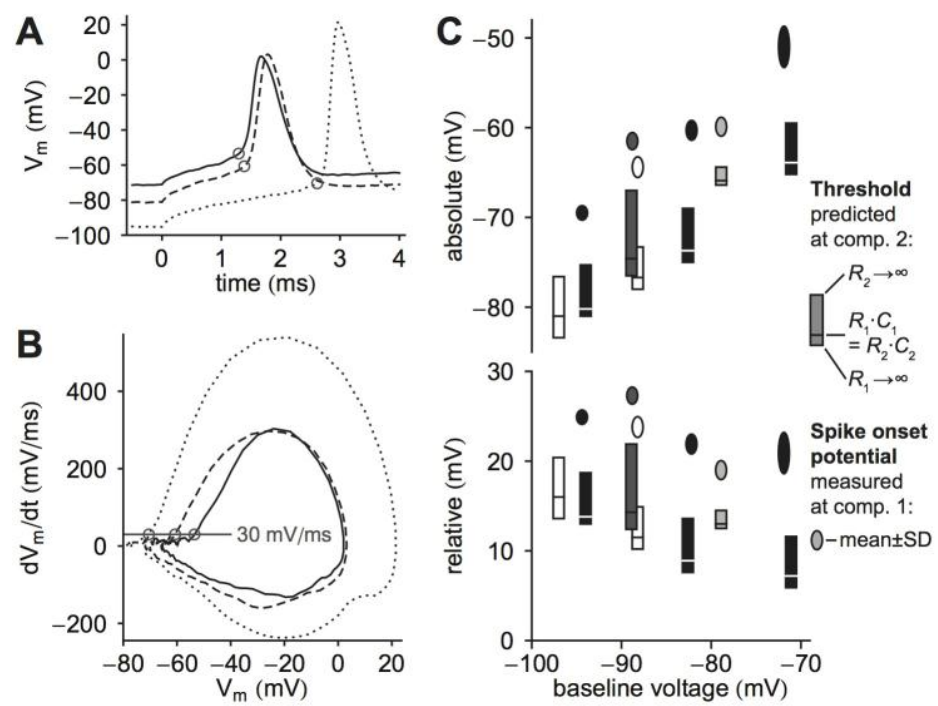

Figure 2.7 Spike threshold and onset potential vary with baseline potential.

A, single SGN responses to a 50 pA current step from 3 different baseline potentials in the same cell.

B, phase plots for the action potentials in A. Open circles in A\&B mark the spike onset potential, defined when slope reached $30 \mathrm{mV} / \mathrm{ms}$.

C, absolute threshold $(\mathrm{mV})$ and threshold relative to baseline potential as determined by the two-compartment model for 4 SGNs (black: cell from 3 baseline potentials; white: cell from 2 baseline potentials; 2 shades of gray: 2 cells from different baseline potentials).

Data analysis and modeling: N.M.C. 


\subsection{Discussion}

Understanding the origin of the neural auditory code in the cochlea requires knowledge about the conversion from neurotransmitter release from the IHC into action potentials in the SGNs, the first neuron in the auditory pathway. Our in vitro data confirmed, as previously suggested in vivo (Siegel 1992), that at low firing rates, almost every neurotransmitter release from the IHC reliably triggers a spike in the SGN. In addition, spikes were generated at the onsets of EPSCs with sub-millisecond precision. We showed that such accuracy can be possible because (1) EPSC amplitudes are far larger than rheobase, (2) SGNs have a short membrane time constant and (3) SGNs have a phasic excitability. Finally the synapse-spike generator complex seems to operate physiologically in a regime where spike latency and jitter are very much reduced to enable faithful sound encoding. Thus, supposedly, the 10-30 SNGs connected to a single IHC transmit to the brain the information about almost every IHC neurotransmitter release in a very robust manner.

\subsubsection{Fast and robust spiking at the origin of the auditory code}

The auditory system relies on the information provided by the auditory nerve fibers for sound source localization. This requires sub-millisecond spiking precision. Such a precision is achieved for example in response to sound tone onset, or during phase locking (Johnson, 1980). Spike timing in the SGNs is determined by a cascade of events, the two last being the integration by the SGN of the neurotransmitter signal from the IHC and spike generation in the SGN. We found that in vitro and at room temperature, the combination of these two last steps yielded a latency of around $0.6 \mathrm{~ms}$ and a jitter of $0.3 \mathrm{~ms}$ (Fig. 2.2). Superposition of multiple EPSCs would produce smaller latencies and jitter.

We found that the system worked in a regime where it was rather resistant to potential biological "fluctuations". The physiological EPSC amplitude $(\approx 300 \mathrm{pA}$ (Grant et al., 2010)) was much larger than rheobase $(\approx 50 \mathrm{pA})$ and increasing the EPSC charge above the physiological mean EPSC charge did not reduce the latency as effectively as it did up to the mean EPSC charge (Fig. 2.4). For repetitions of an EPSC shapes with close to physiological total charge, jitter was close to zero ( $\leq 23 \mu \mathrm{s})$. Finally, small changes in the baseline potential didn't strongly affect latencies or reliability (Fig. 2.3). Indeed, rheobase and threshold were compensated by homeostatic mechanisms (Fig. 2.7). Our results suggest that the jitter in spike timings arose mainly from the variability in 
neurotransmitter release time-course, rather than from the spike generation mechanism itself.

Spike latencies in response to physiological EPSCs could be well predicted by a leaky integrate-and-fire neuron with very short effective time constants (1-2 ms) and a very sharp spike initiation $\left(\Delta_{T h} \approx 1-2 \mathrm{mV}\right)$ for the exponential leaky integrate-and-fire neuron (Fig. 2.5). This indicates a very responsive neuron with a fast spike generation mechanism. In addition this model provided an easy way to predict the spike latency for a given EPSC shape and thus for this particular stage of auditory processing. More physiological models might be required to predict the neuron response to higher EPSC rates (Brette and Gerstner, 2005; Herz et al., 2006).

\subsubsection{Phasic excitability of the spiral ganglion neuron}

We found that the responses of postsynaptic boutons of type I SGNs innervating IHCs in the cochlear apex are highly phasic - firing once, or very rarely twice (Fig. 2.3) in response to steady depolarizing currents. This class III excitability property probably result from the low-voltage activated $\mathrm{K}^{+}$currents (Mo et al., 2002; Szabó et al., 2002; Prescott et al., 2008a) and resulted in the fact that latencies longer than 3 ms were rarely observed in response to synaptic events or EPSC-like stimuli (Fig. 2.2, 2.4, 2.5, and 2.6). Similar results were obtained with recordings from the endbulbs of Held (Lin et al., 2011) the presynaptic terminals formed by SGNs onto the spherical bushy cells in the brainstem's cochlear nucleus. This phasic property could be important in at least two aspects for sound encoding. First, the arrival of a single EPSC triggers just a single spike. In fact, blocking DTX-sensitive $\mathrm{K}+$ currents in medial nucleus of the trapezoid body (MNTB) neurons removed their phasic nature and made them spike multiple times in response to a single EPSC (Brew and Forsythe, 1995). This would relay to the brain information unrelated to the EPSC onset. Second, the phasic property allows the neuron to spike only at stimulus onset and prohibits long latencies. This restricts the neurons response to the arrival of the neurotransmitter release from IHC. Phasic spiking neurons are found all along the auditory pathway and in many other sensory systems (Prescott et al., 2008a).

This SGN bouton spiking behavior closely resembles the phasic or rapidlyadapting class reported for isolated SGN somata (Mo and Davis, 1997; Mo et al., 2002; Lv et al., 2010). But the firing properties of cultured SGNs were heterogeneous within and between studies and SGNs were sometime found not to be phasic (Rusznák and Szucs, 2009). But neuron excitability can be different at axons and boutons, in 
comparison to somata (Shu et al., 2007a; Rancz et al., 2007). These two studies demonstrate that the soma can be tonic, whereas the axon or bouton phasic.

We found additional differences between our acute preparation and cultured somata. First, spike latencies and spike widths were less than $10 \mathrm{~ms}$ and around $0.5 \mathrm{~ms}$, respectively, as opposited to more than $50 \mathrm{~ms}$ and $4-5 \mathrm{~ms}$ respectively. Second, the resting membrane potential was in our case $-72 \pm 6 \mathrm{mV}$ (mean \pm s.d., $\mathrm{n}=11$ ), relatively hyperpolarized in comparison to most previous studies, which ranged from approximately -54 to $-77 \mathrm{mV}$ (Rusznák and Szücs, 2009). Third, we also found the relative spiking threshold to be smaller. In conclusion, our preparation showed a more excitable and rapidly responding behavior, suitable for auditory encoding.

SGNs firing characteristics (Lin, 1997; Mo and Davis, 1997b; Adamson et al., 2002; Liu and Davis, 2007; Flores-Otero et al., 2007; Lv et al., 2010), as well as IHC exocytosis properties (Liberman, 1982; Merchan-Perez and Liberman, 1996; Johnson et al., 2008; Frank et al., 2009; Meyer et al., 2009) may depend on the tonotopical position and on the location of IHC innervation. Our recordings were performed of the boutons on the modiolar or neural face of IHCs and in the apex of the cochlea. Dependence of SGN properties on the two above mentioned factors remain to be studied.

\subsubsection{Synapse-spike initiation coupling}

The positioning and the size of the axonal initial segment (AIS: place where the action potential is generated) determine and modulate the excitability of a neuron (Kuba et al., 2006; Hu et al., 2009; Kuba et al., 2010; Fleidervish et al., 2010; Grubb et al., 2011). In the unique geometrical micro-machinery at the SGN, the postsynapse is directly linked to the AIS, before the soma.

Three pieces of evidence argue that the presynapse is close to the spike generator zone. First the large slope of the spikes indicates that a high concentration of sodium channels is present in the close proximity (Fig. 2.2). Second, the shape of the spike rise (after the EPSP) exhibited only one component, in contrast to cortical neurons soma recordings (Shu et al., 2007b; Hu et al., 2009), which exhibit two: one produced by the activation of $\mathrm{Na}^{+}$channels in the soma and one by those in the axon. Finally the extremely short minimal delays $(<250 \mu \mathrm{s})$ for spike onset observed in SGNs. It still remains to be investigated if the spike is initiated as the heminode of the first node of Ranvier. Thus, synapse and axonal spike generator, both separated by only 20-30 $\mu \mathrm{m}$ (Hossain et al., 2005), form a compact sound-encoding element to support efficient and precise transmission. 


\subsubsection{Consequences on SGN in vivo functional heterogeneity}

The origin of auditory nerve fibers' heterogeneity is a long-standing question in the auditory system field (Kiang, 1965; Taberner and Liberman, 2005). In vivo, auditory nerve fibers are known to greatly vary in terms of spontaneous rate, threshold and sensitivity. Recently it has been proposed that SGN property variability comes from the fact that each SGN receives EPSCs of different waveforms (Grant et al., 2010). However, in contrast to the immature SGNs (Yi et al.,2010), our recordings indicate that nearly all EPSCs triggered spiking. In addition, the low rheobase would suggest that a high spike success rate would be common to all the investigated SGNs ( $n=8)$. If all SGNs in the cochlea would have similar excitability properties, we would be driven to argue that the reported in vivo SGN heterogeneity in terms of spontaneous rate and sensitivity is mainly presynaptic, as the relationship between neurotransmitter release and spiking is nearly one to one.

On the other hand, our data does suggest that SGNs receiving on average different EPSC waveforms, could exhibit slightly different latencies and maybe even jitter (Fig. 2.4, 2.5, 2.5). Therefore, a difference in spike timing statistics might be apparent (Heil et al., 2007). To understand the consequences of different EPSC waveforms on the input-output relationship of this synapse, further work should be performed by applying injections of EPSC trains into the SGN bouton. 


\subsection{Materials and Methods}

\subsubsection{Preparation and electrophysiology}

Full experimental methods in (Rutherford, Chapochnikov and Moser, unpublished). Most important information relevant for data analysis and modeling in short below.

Experiments were done in inner ears of P11-P19 Wistar rats. Doing the intracellular recording to a bouton contacting the base of an inner hair cell (for method, see (Grant et al., 2011), Experiments were performed at room temperature $\left(\approx 22-24^{\circ} \mathrm{C}\right)$. Electrode resistance was 6-10 $\mathrm{M} \Omega$. The liquid junction potential of $6 \mathrm{mV}$ was compensated online. Voltage and current signals were at 20 microsecond intervals. Current-clamp stimuli where not filtered on the path to the cell. The voltage signal in current-clamp is filtered with a time constant equal to the access resistance $\left(\mathrm{R}_{\mathrm{a}}\right)$ times the uncompensated patch-pipette capacitance $\left(\mathrm{C}_{\text {fast, }}\right.$, residual $\left.\approx 1 \mathrm{pF}\right)$. Pipette capacitance compensation ( $\mathrm{C}_{\text {fast }} \approx 5-7 \mathrm{pF} ; \tau \approx 1-2 \mu \mathrm{s}$ ) was set in voltage-clamp and adjusted $-5 \%$ when switching to current-clamp. No bridge-balance or active series resistance compensation was used. Current signals were low-pass filtered at $5 \mathrm{kHz}$ (4-pole Bessel). Current-clamp stimuli $\left(\mathrm{I}_{\text {ini.) }}\right.$ ) are displayed as measured from the current monitor.

Input resistances ranged from 200 to $1000 \mathrm{M} \Omega$ between cells when measured with small current or voltage steps near the zero-current potential, which ranged from -70 to $80 \mathrm{mV}$ between cells (electrophysiological parameters in Table 2.1). Data from 12 boutons ( 2 pre-, 10 post-hearing onset) were considered sufficient in quality and duration to be analyzed for this study. When present, spontaneous activity was recorded in voltage-clamp and current-clamp modes prior to eliciting spikes with defined excitatory current waveforms from one or more baseline potentials.

In addition to current-clamp experiments from the zero-current potential, we also set the membrane potential to relatively depolarized or hyperpolarized levels by applying steady holding currents. The membrane potential sometimes shifted by $\pm 3 \mathrm{mV}$ while holding at constant current over the duration of a 15 - 90 minute recording. Data were acquired over successive 20 - $60 \mathrm{~s}$ periods centered around mean baseline potentials in interleaved sequence (e.g., -93, -70, -82, and $-102 \mathrm{mV}$ with holding currents of $-20,7,-6$, and $-29 \mathrm{pA}$, respectively).

\subsubsection{Data analysis}

IGOR (Wavemetrics), MiniAnalysis (Synaptosoft), and Mathematica (Wolfram Research) software were used for analysis and plotting. All sweeps were inspected visually. Spikes 
could be unambiguously discriminated from EPSPs by their peak amplitude. When studying responses to current-clamp stimuli we discarded sweeps with spontaneous activity within $20 \mathrm{~ms}$ prior to or during the stimulus. For analysis of spontaneous activity, we discarded $20 \mathrm{~s}$ segments with $> \pm 2 \mathrm{mV}$ deviations from the intended baseline potential. Except for the display in Fig. 2.4A, all voltage traces were corrected by subtracting the voltage error due to series resistance $\left(V_{\text {error }}\right)$ equal to the injected current ( $\mathrm{I}_{\text {inj. }}$ ) multiplied by the access resistance $\left(\mathrm{R}_{\mathrm{a}}\right)$. EPSPs and EPSCs were detected in MiniAnalysis (Fig. 2.1) by setting an amplitude threshold of $\approx 7 \mathrm{X}$ the root mean square (rms) noise. For example, in one voltage-clamp recording the rms noise was $1.4 \mathrm{pA}$ and the amplitude threshold for EPSC detection was $10 \mathrm{pA}$. Because of the large event size relative to the noise, the counting of events was relatively insensitive to the detection threshold: for example, in a $60 \mathrm{~s}$ recording segment, changing amplitude threshold from 10 to $5 \mathrm{pA}$ resulted in detection of 4 additional events (from 319 to 323) and going down to $2.5 \mathrm{pA}$ resulted in detection of 6 more events.

To make accurate and precise estimates of spike onset time that didn't depend strictly on the sampling frequency, we first measured when $V_{m}$ depolarized to $10-30 \mathrm{mV}$ below the spike peak (depending on the mean spike height of that recording) using linear interpolation between adjacent sampling points. We then subtracted $0.08-0.15 \mathrm{~ms}$ (depending on the average spike width of that recording) from the measured spike time. This estimated spike onset time (or spike onset latency) corresponded to the beginning of the spike upstroke relative to stimulus onset. In the case of spontaneous spikes (i.e., in response to a neurotransmitter release event from the IHC), stimulus onset was defined when the first of either criteria was met: the voltage slope exceeded $5 \mathrm{mV} / \mathrm{ms}$, or (for slow EPSPs) the voltage value increased $>2 \mathrm{mV}$ above the baseline potential. This measurement of the timing of neurotransmitter release events for slow EPSPs could have led to an underestimate of the mean and s.d. of IHC-evoked spike onset latency. However, the size of this uncertainty is small relative to the differences in latency we observed as a function of EPSP waveform (Fig. 2.3), and the expected directional trend of such uncertainty would not affect our interpretation of comparisons between IHCevoked and current-clamp evoked (CC-evoked) responses (Fig. 2.3,2.6,2.8).

The maximum EPSP slope was determined as the local maximum in the voltage temporal derivative before the local maximum due to the spike upstroke. In cases when no local maximum existed, we measured the slope at the time of the global minimum of the second derivative before the spike upstroke, which corresponded to when the prepotential slope increased the least and occurred just before the spike onset. Discrete 
temporal derivatives of voltage were calculated using central differences. Data is reported as mean \pm standard deviation (s.d.).

\subsubsection{EPSC-like shapes}

EPSC-like current waveforms were constructed by specifying their charge, linear risetime, plateau duration, and exponential decay time constant. Necessary amplitudes were calculated. For the EPSC-like shapes 1-4 (Fig. 2.6), stimuli where presented in order of increasing amplitude for each shape separately in the sequence 2, 3, 1, 4. Repetitions were looped after all of the shapes and amplitudes were presented. Stimulation from different baseline potentials was delivered in adjacent recording segments. Systematically varied EPSC-like shapes (see Fig. 2.7-2.8) were presented with nested loops: the outer loop was for rise-times 0.1 - $1.5 \mathrm{~ms}$ in steps of $0.1 \mathrm{~ms}$; the next loop was for charges $100-700 \mathrm{fC}$ in steps of $100 \mathrm{fC}$; the inner loop was for plateau durations $0-5 \mathrm{~ms}$ in steps of $0.5 \mathrm{~ms}$. Stimuli were delivered at $10 \mathrm{~Hz}$.

\subsubsection{Two-compartment model}

We averaged the initial voltage responses to small subthreshold depolarizing current steps and fitted them to the peak (first 3 to $10 \mathrm{~ms}$, which depended upon the baseline potential) with a double exponential curve:

$$
f(t)=V_{0}-I\left(\left(R_{\text {fast }}+R_{a}\right) e^{-t / \tau_{\text {fat }}}+R_{\text {slowe }} e^{-t / \tau_{\text {slaw }}}\right)
$$

where $I$ is the difference between the injected current and the holding current, $R_{a}$ is the access resistance measured in voltage-clamp, and $V_{0}$ is the voltage of the fit function at steady-state (e.g., Fig. 2.4E). This provided two time constants, $\tau_{\text {fast }}$ and $\tau_{\text {slow }}$, with the corresponding resistances $R_{\text {fast }}+R_{a}$ and $R_{\text {slow }}$, respectively. The values for $\tau_{\text {fast }}, \tau_{\text {slow }}, R_{\text {fast }}$, and $R_{\text {slow }}$ ranged between $0.07-0.4 \mathrm{~ms}, 0.7$ - $4.0 \mathrm{~ms}, 40$ - $120 \mathrm{M} \Omega$, and 220 - $470 \mathrm{M} \Omega$, respectively, depending on the recording. Within those ranges, larger values were found when $V_{\text {base }}$ was more hyperpolarized. These values were used to construct an electrical circuit composed of 2 compartments, characterized by 3 resistances: $R_{1}, R_{2}$, and $R_{a x i a l}$, and 2 capacitances: $C_{1}$ and $C_{2}$ (Fig. 2.7B). $R_{\text {axial }}$ is the resistance connecting the 2 compartments. The electrode is connected to compartment 1 . The membrane potentials at each compartment are described by the following system of ordinary differential equations: 


$$
\left\{\begin{array}{l}
\mathrm{R}_{1} C_{1} \frac{d V_{1}(t)}{d t}=-\left(V_{1}(t)-V_{\text {base }}\right)-\frac{\mathrm{R}_{1}}{\mathrm{R}_{\text {axial }}}\left(V_{1}(t)-V_{2}(t)\right)+\varphi_{1}\left(V_{1}(t)\right)+\mathrm{R}_{1} I(t) \\
\mathrm{R}_{2} C_{2} \frac{d V_{2}(t)}{d t}=-\left(V_{2}(t)-V_{\text {base }}\right)-\frac{\mathrm{R}_{2}}{\mathrm{R}_{\text {axial }}}\left(V_{2}(t)-V_{1}(t)\right)+\varphi_{2}\left(V_{2}(t)\right)
\end{array}\right.
$$

where $V_{\text {base }}$ was the measured average over $1 \mathrm{~ms}$ before stimulus onset, and $I(t)$ is the difference between the injected current and the holding current at time $t . V_{i}$ is the voltage at compartment i. $\varphi_{1}(V)$ and $\varphi_{2}(V)$ are here both 0 and will be used later. Differential equations were solved with a $9^{\text {th }}$ order explicit Runge-Kutta scheme implemented in the method NDSolve in Mathematica 7.0.1.

An infinite number of two-compartment circuits can reproduce the doubleexponential voltage response measured at the bouton because there are 4 measured parameters and 5 defining the circuit. To estimate the range of possible membrane potentials at the $2^{\text {nd }}$ compartment, three scenarios were considered (see Fig. 2.9C). In the first, most conservative scenario (used for Fig. 2.7,2.8), we assumed that the specific membrane resistance and capacitance are the same in both compartments. Then $R_{1} C_{1}=$ $\mathrm{R}_{2} C_{2}=\tau_{\text {slow }}$ and the amplitude of the slow component response is the same in both compartments. In the second scenario we assumed $R_{2}$ to be infinity (Mennerick et al., 1995). In this case, no current flows to the second compartment at steady state, which results in no voltage difference between the 2 compartments. In the third scenario, we assumed $R_{1}$ to be infinity, which results in the largest voltage difference between the 2 compartments at steady state. For these 3 scenarios, the compartment parameters were deduced analytically from the measured $\tau_{\text {fast}}, \tau_{\text {slow }}, R_{\text {fast, }}$ and $R_{\text {slow. }}$ In all 3 scenarios:

$$
C_{1}=\tau_{\text {fast }} \tau_{\text {slow }} / A
$$

where $A=R_{\text {slow }} \cdot \tau_{\text {fast }}+\mathrm{R}_{\text {fast }} \cdot \tau_{\text {slow }}$.

For the first scenario $\left(R_{1} C_{1}=R_{2} C_{2}\right)$ :

$$
\mathrm{R}_{1}=A / \tau_{\text {fast }} ; \mathrm{R}_{2}=\frac{\mathrm{R}_{\text {slow }} \cdot A}{\mathrm{R}_{\text {fast }} \cdot \tau_{\text {slow }}} ; C_{2}=\frac{\mathrm{R}_{\text {fast }} \cdot \tau_{\text {slown }}^{2}}{\mathrm{R}_{\text {slow }} \cdot A} ; \mathrm{R}_{\text {axial }}=\frac{A^{2}}{\mathrm{R}_{\text {fast }} \cdot \tau_{\text {slow }} \cdot \delta \tau}
$$

where $\delta \tau=\tau_{\text {slow }}-\tau_{\text {fast }}$.

For the second scenario $\left(R_{2} \rightarrow \infty\right)$ :

$$
\mathrm{R}_{1}=S ; C_{2}=\frac{P \cdot \delta \tau^{2}}{S^{2} \cdot A} ; R_{\text {axial }}=\frac{A^{2} \cdot S}{P \cdot \delta \tau^{2}}
$$

where $S=R_{\text {fast }}+R_{\text {slow }}$ and $P=R_{\text {fast }} \cdot R_{\text {slow }}$. 
For the third scenario $\left(R_{1} \rightarrow \infty\right)$ :

$$
C_{2}=\frac{B^{2}}{A \cdot P \cdot \delta \tau^{2}} ; R_{2}=\frac{P \cdot \delta \tau^{2}}{B} ; R_{\text {axial }}=\frac{A^{2}}{B} ;
$$

where $B=R_{\text {slow }} \cdot \tau_{\text {fast }}^{2}+R_{\text {fast }} \cdot \tau_{\text {slow }}^{2}$.

SGN voltage responses did not appear entirely linear, but the responses to 5 - 20 $\mathrm{pA}$ steps were most linear from $V_{\text {base }}=-95 \pm 5 \mathrm{mV}$, so results in the text for $\tau_{\text {fast, }} \tau_{\text {slown }}$ $R_{\text {fast }}, R_{\text {slow, }}$ and the solutions for the two-compartment circuit were based upon those responses, assuming the first scenario (above). Using voltage-clamp traces, the twocompartment model provided an estimate of $R_{a}$ (Pandey and White, 2002). This estimate (34 $\pm 6 \mathrm{M} \Omega ; \mathrm{n}=8$ ) was insignificantly smaller than the estimate based upon measurement using a one-compartment model of the data (Table 2.1).

\subsubsection{Leaky integrate and fire models}

First, we considered the leaky integrate-and-fire neuron model (LIF; (Lapicque, 1907; Stein, 1967; Knight, 1972)). The evolution of the voltage is passive, as described in equation (2.2) with $\varphi_{1}(V)=\varphi_{2}(V)=0$. A spike is emitted with a fixed delay $D$ after the membrane potential $V_{i}(t)$ reached threshold $V_{T b}$. To approximate effects of the timecourse of voltage-gated $\mathrm{Na}^{+}$channel activation, we also considered the exponential leaky integrate-and-fire neuron model (EIF; (Fourcaud-Trocmé et al., 2003). The evolution of the potential is given in (2.2) with $\varphi_{i}(V)=\Delta_{T} \cdot \exp \left(\left(V-V_{T}\right) / \Delta_{T}\right)$ in the active compartment, and $\varphi_{i}(V)=0$ in the passive compartment. $V_{T}$ is the maximum steadystate voltage at which the active compartment can remain without spiking in the presence of a constant injected current, and $\Delta_{T}$ is the spike slope factor which characterizes the sharpness of spike initiation (i.e. the voltage range over which $\mathrm{Na}^{+}$channels activate). Due to the supralinear function $\varphi_{i}(V)$, the membrane potential diverges to infinity in a finite time once enough current is injected. A spike occurs with a fixed delay $D$ after the membrane potential $V_{i}(t)$ reached $V_{T}+10 \cdot \Delta_{T}$ in the active compartment. For $\Delta_{T}=0$ $\mathrm{mV}$, the EIF model reduces to the LIF. For the LIF, the voltage at the first compartment is always higher than the voltage as the second, because the current is injected in the first compartment. Therefore having the spike generator only in the first compartment is equivalent to having it in both, if one assumes the same threshold voltage $V_{T b}$ in both compartments. For the EIF, having the spike generator only in the first compartment would imply that only the first compartment is active, which is an unreasonable 
assumption. Therefore we added the LIF or EIF mechanism to either the $2^{\text {nd }}$ or both compartments.

For each cell from a particular baseline potential, in the LIF model the only free parameter to assign was threshold $V_{T h}$. For the EIF, there were two parameters: $V_{T}$ and $\Delta_{T}$. We determined these parameters so that the models gave the best predictions of the data, in terms of both spike occurrence and latency. Using the voltage responses to hundreds of different EPSC-like stimuli, we calculated the "raw" latencies $\left(\mathrm{R}_{i}\right)$ to threshold potential $V_{T b}$ (for the LIF) or to $V_{T}+10 \cdot \Delta_{T}$ (for the EIF). Then, for the $n$ spikes predicted by the model and existing in the recording, we calculated the rms latency prediction error $\delta L$ :

$$
\delta L=\sqrt{\frac{1}{n} \sum_{i=1}^{n}\left(L_{i}-P_{i}\right)^{2}}
$$

where $L_{i}$ are the measured latencies and $P_{i}$ are the predicted latencies, defined as $P_{i}=R_{i}$ $+D$ (see Fig. 2.7C,D). $D$ is the fixed delay we added to all the "raw" latencies $R_{i}$ to obtain the predicted latencies $P_{i}$ so that the mean prediction error was zero:

$$
D=\frac{1}{n} \sum_{i=1}^{n} L_{i}-R_{i} \Rightarrow 0=\frac{1}{n} \sum_{i=1}^{n} L_{i}-P_{i}
$$

With the spike generator in the second compartment, $D$ ranged from 0.0 to $0.27 \mathrm{~ms}$ for the LIF and from -0.11 to $0.10 \mathrm{~ms}$ for the EIF. $D$ was determined to minimize the mean $\delta L$ for each cell- $V_{\text {base }}$ combination. In some cases a negative value was found for $D$ because the actual spike onset occurred before the voltage in the EIF model reached $V_{T}$ $+10 \cdot \Delta_{T}$. We also calculated the fraction of correctly-predicted spike occurrences, $F$, equal to $1-(E+M) / N$, where $E$ is the number of extra spikes predicted by the model, $M$ is the number of missed spikes, and $N$ is the total number of stimuli.

To find the best $V_{T h}$ for the LIF model, we calculated $F$ and $\delta L$ at $0.1 \mathrm{mV}$ steps for $V_{T h}$ between $V_{\text {base }}$ and $V_{\text {base }}+50 \mathrm{mV}$. To find the best $V_{T}$ and $\Delta_{T}$ for the EIF model, we calculated $F$ and $\delta L$ at $0.1 \mathrm{mV}$ steps for $V_{T}$ between $V_{T b}-5 \mathrm{mV}$ and $V_{T b}+5 \mathrm{mV}$, and for $\Delta_{T}$ between 0 and $5 \mathrm{mV}$. Percent improvement was calculated as $100 \cdot[1$ $\delta L\left(\right.$ model $\left.\left._{1}\right) / \delta L\left(\operatorname{model}_{2}\right)\right]$, then compared against zero. Statistical significance was assessed with the Z-test. 


\section{The synaptic ribbon as a Brownian conveyor trap}

\section{Short summary}

In chapter 2 we quantitatively showed how spiral ganglion neurons were specialized to precisely read out the timing of nearly every neurotransmitter release coming from the inner hair cell ribbon synapses. However, even though EPSCs can have very large amplitudes (up to $800 \mathrm{pA}$ ), they cannot elicit more than one spike in the spiral ganglion neurons due to the neuron's phasic excitability. Thus, when spiral ganglion neurons have steady state firing rate of up to $400 \mathrm{~Hz}$, at least 400 vesicles exocytose per second from one single ribbon synapse. This imposes an extremely high demand on the vesicle cycle and especially on vesicle ready releasable pool replenishment. In this chapter we simulated vesicle diffusion on the synaptic ribbon. We proposed a mechanism by which the ribbon's presence might facilitate very high vesicle replenishment rates and thus enhance the synapse's coding capability. 


\subsection{Introduction}

Chemical synapses are specialized secretion machineries that form the basis of neuron-toneuron communications. Many processes require cooperation to turn this secretion machinery into a reliable information transmission device (Haucke et al., 2011). First vesicles have to be produced and filled with neurotransmitter, the signaling molecule that will bind to the postsynapse and trigger an electrical current. Second, vesicles have to approach the right place of exocytosis, right opposite the postsynaptic receptor cluster. Finally vesicles have to be secreted at the right moment, as timing is one of the key elements of neural communication. In this study, we are going to address the second step mentioned above.

During strong sustained exocytosis, high demands are imposed on each step of the vesicle cycle (Neher, 2010). In particular, vesicle transport logistics must be very efficient so that enough vesicles reach the synaptic active zone (AZ) - the location of the vesicle release sites. The AZ has three main constituents (Zhai and Bellen, 2004): the cell membrane, the cytomatrix inside the cell membrane where vesicles dock and electrondense structures projecting into the cytoplasm which tether vesicles. The size and shape of the electron-dense structures determine the number of vesicles maintained near the AZ and seem to be correlated with the exocytosis demands of a particular synapse. For example, sensory cells in the ear and in the eye, that exocytose vesicles continuously, have very extended electro-dense structures: synaptic ribbons (Matthews and Fuchs, 2010). Even though ribbons allow the storage of a large number of vesicles, it is not clear yet whether such a storage enables vesicles to access the AZ faster, in comparison for example to a ribbon-less synapse filled with vesicles. In fact, vesicle tethering to the ribbon might significantly decrease vesicle mobility and hinder fast replenishment rates.

Using realistic simulations of vesicle diffusion on the ribbon surface, we found that the ribbon might actually accelerate vesicle arrival to the AZ: by functioning as a "Brownian conveyor trap". The ribbon tethers vesicles on its surface and thus establishes a high lateral pressure in the vesicle arrangement. As soon as a vesicle is exocytosed and frees a space, the thermal pressure due to the Brownian motion of vesicles pushes vesicles toward the release sites. In particular, we found that replenishment rates increase exponentially with vesicle packing density. Thus an electron-dense structure as the synaptic ribbon might not only store a halo of vesicles close to the $\mathrm{AZ}$, but also provide the means for accelerated replenishment of the release sites. 


\subsection{Results}

\subsubsection{Model of vesicle diffusing on the ribbon surface}

The synaptic ribbon might equip ribbon synapses with the ability to sustain prodigiously high exocytosis rates (Matthews and Fuchs, 2010). It remains however debated if the ribbon does indeed fulfill such a role (Jackman et al., 2009), and if it does, through which mechanism (Parsons and Sterling, 2003). As vesicles are assumed to access the AZ release sites mainly via movement on the ribbon surface (Zenisek et al., 2000; Holt et al., 2004; LoGiudice and Matthews, 2009), to better understand the mechanisms replenishment of the vesicle ready releasable pool (RRP), we modeled the Brownian motion of vesicles tethered to the ribbon (Fig. 3.1a-c, Methods). The impact of ribbon tethering on vesicle mobility is not well understood - depending on the tethering force properties, vesicle motion might or might not be slowed down. We assumed the vesicle diffusion coefficient ( $D=50 \mathrm{~nm}^{2} / \mathrm{ms}$ for a $40 \mathrm{~nm}$ diameter vesicle) 10 times smaller than predicted for the cytoplasm (500 nm²/ms) (Luby-Phelps et al., 1987), as a conservative estimate. In the first model, vesicles diffused vertically in a one-dimensional column of $300 \mathrm{~nm}$ high, perpendicular to the AZ (Fig. 3.1a, 1D model). This model would apply if vesicle motion was restricted to vertical tracks on the ribbon surface. In a second model, vesicle moved on a two-dimensional cylindrical surface of $300 \mathrm{~nm}$ high and $500 \mathrm{~nm}$ circumference, representing the entire ribbon surface (Fig. 3.1b, 2D model)(Cichocki and Hinsen, 1990). Forces at the top and bottom boundaries constrained vesicles to stay inside the simulation volume.

\subsubsection{High vesicle packing density immobilize vesicles}

We first studied how the vesicle packing density $\varphi$ on the ribbon surface affects vesicle mobility, when observed over different time intervals (Fig. 3.2a-b). In the synapse, vesicle densities on the ribbon could be set by the concentration of vesicles in the cytoplasm as well as by the "stickiness" of the ribbon tethers. Vesicle mobility was assessed with the apparent diffusion coefficient $D_{a p p}$. At very short observation intervals $\left(0.01 \mathrm{~ms}\right.$ ), vesicles moved almost freely $\left(D_{\text {app }} \approx D\right)$; over longer time intervals (up to 10 ms) however, vesicles in high packing density $\left(\varphi_{2 D} \approx 60 \%\right)$ appeared practically immobile: they fluctuated locally, but were trapped in a quasi-crystallized arrangement. These results are consisted with previous predictions (Cichocki and Hinsen, 1990) (Gaffield et al., 2006). In absence of synapse stimulation, the vesicle packing on the ribbon is extremely high $\left(\varphi_{2 D} \approx 80 \%\right.$ ) (Lenzi et al., 2002), approaching the theoretical 
limit of $90 \%$ (hexagonal packing). But it could decrease to $\varphi_{2 D} \approx 40 \%$ during strong stimulation. In summary, vesicle crowding is a critical determinant of vesicle mobility.

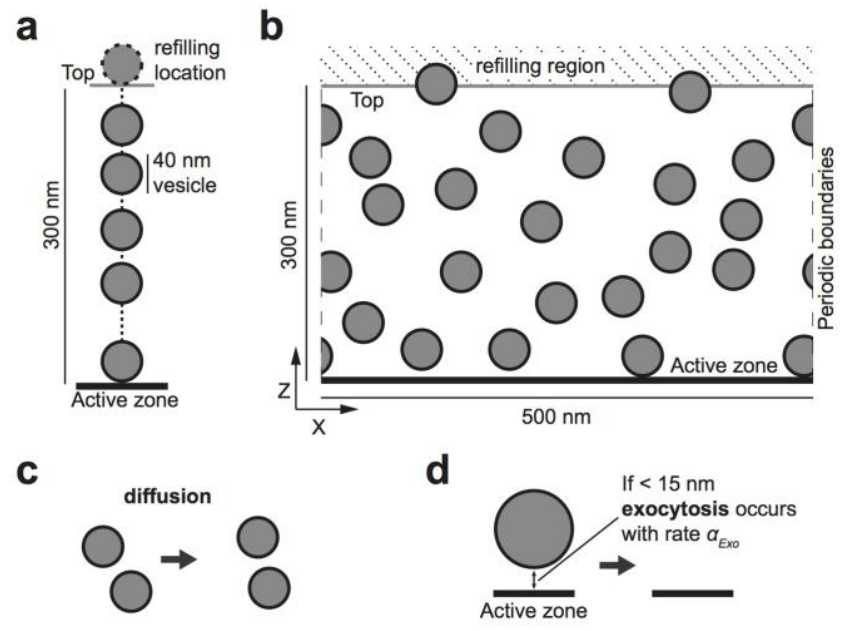

Figure 3.1: Simulation scheme

(a) scheme of the one-dimensional simulation column.

(b) scheme of the two-dimensional simulation cylinder.

(c) vesicles diffuse without overlapping.

(d) vesicles are released with a rate $\alpha_{\text {Exo }}$ when closer than $15 \mathrm{~nm}$ to the active zone (AZ). The exocytosis reaction could occur when a vesicle approached the AZ by closer than $15 \mathrm{~nm}$. Exocytosis resulted in vesicle disappearance from the simulation volume. a

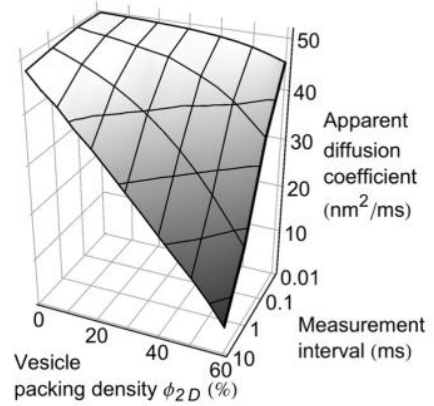

b

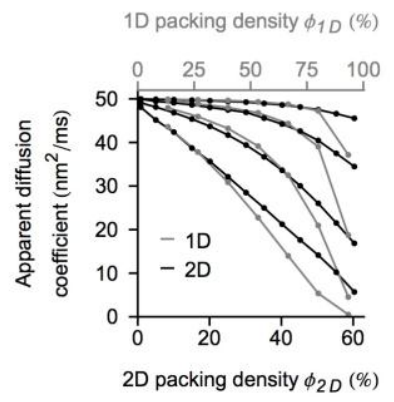

Figure 3.2: Mobility of vesicles in the absence of release

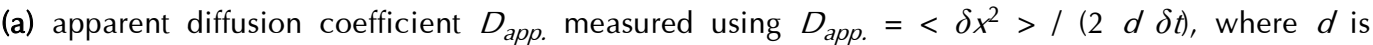
the number of dimension of the system, $\delta x$ is the displacement performed over a measurement time $\delta t$ and $\langle$.$\rangle is the averaging over displacements, vesicles and simulations. Here for the$ 2D simulation volume. For high packing densities and long measurements intervals vesicles appear virtually immobile.

(b) same as in (e), for both 1D and 2D simulations volumes, for measurement times 0.01, 0.1, 1 and $10 \mathrm{~ms}$. The 1D vesicle packing density $\varphi_{1 D}$ was calculated as the space taken by the sum of vesicles diameters divided by the height of the space. The conversion from $\varphi_{1 D}$ to $\varphi_{2 D}$ was done assuming that the 1D column in $50 \mathrm{~nm}$ wide.

The forces on the top and the bottom of the simulation were $10 \mathrm{pN}$. Vesicles where placed randomly in the simulation volume and given $100 \mathrm{~ms}$ to equilibrate.

For 1D we used simulations of 200 to $1000 \mathrm{~s}$ duration, depending on the packing density. For $2 \mathrm{D}$, we used simulations of 30 to $2000 \mathrm{~s}$.

\subsubsection{High vesicle packing on the synaptic ribbon enables efficient vesicle replenishment}

Next, to examine the consequences of vesicle crowding on RRP (ready releasable pool) replenishment, we set a very high vesicle exocytosis rate $\left(\alpha_{E \times 0}=100 \mathrm{~ms}^{-1}\right)$ at the AZ. As a 
result RRP replenishment was the rate-liming step of the vesicle cycle and the resulting exocytosis rates were used to compute replenishment rates. Vesicle exocytosis, leading to its disappearance from the simulation volume, could occur only when the vesicle approached the AZ by less than $15 \mathrm{~nm}$. (Fig. 3.1d). A steady packing density was maintained by adding vesicles at the top of the ribbon, representing capturing of cytosolic vesicles. Adding vesicles at other locations on the ribbon would further accelerate RRP replenishment. Histograms in Fig. 3.3a,b show that inter-exocytosis-intervals are essentially exponentially distributed, indicating a Poisson process. In 1D a refractory period is present, due to the finite diffusion time a vesicle need to reach the AZ, following the exocytosis of a preceding vesicle.

Replenishment rates and mean vesicle top-down velocities increased exponentially with packing density (Fig. 3.3c,d), demonstrating a crucial role of high vesicle packing for efficient replenishment. Replenishment rates in contrast would increase only linearly with the diffusion coefficient $D$. In fact, the mean square displacement $\left\langle\delta x^{2}\right\rangle=2 d D \delta t$, where $d$ is the number of system's dimensions and $\delta t$ is the time interval. Thus doubling the diffusion coefficient would make the system behave twice faster. Replenishment rates were insensitive to the mechanism through which a mean vesicle packing density was achieved (Fig. 3.3c, crosses: vesicles were added to the simulation volume as soon as a fixed volume on the top of the simulation volume was vesicle-free). The rates of replenishment per release site $(40-200 \mathrm{~Hz})$ for packing densities between 40 and $60 \%$ were consistent with the range of maximum possible replenishment rates found at ribbon synapses (70 Hz, (Pangrsic et al., 2010)). During such massive exocytosis, vesicles would move down at mean velocities up to $10 \mathrm{~nm} / \mathrm{ms}$. The whole vesicle pool of a $200 \mathrm{~nm}$ height ribbon would then be exocytosed in about $20 \mathrm{~ms}$, as suggested by (Edmonds et al., 2004).

In conclusion, although a strong vesicle crowding on the synaptic ribbon leaves vesicles virtually immobile in absence of release, during vesicle release it can enable ultrafast vesicle movement towards the AZ. This suggest a general function of the ribbon as a "Brownian conveyor trap": the tethers trap vesicles on the ribbon surface, generating a high self-organized lateral thermal pressure, which pushes vesicles to the AZ and enables rapid replenishment of the release sites. 
a

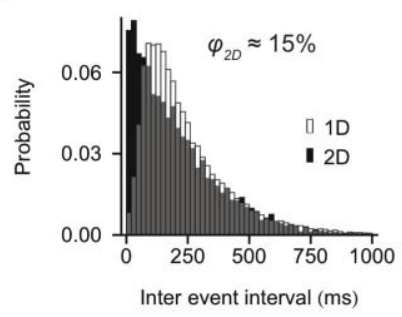

C

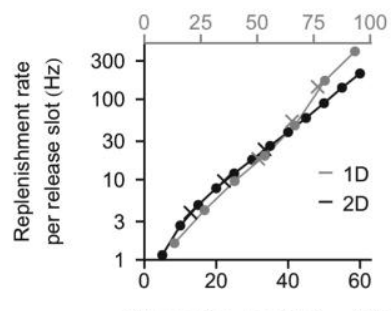

b

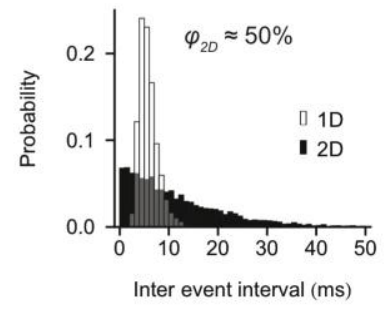

d

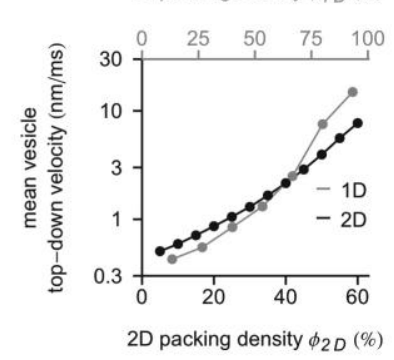

Figure 3.3: Impact of vesicle crowding on vesicle replenishment rates

(a),(b) Inter-exocytosis intervals per release slot by fixing $\alpha_{\text {exo }}=100 \mathrm{kHz}$, estimating the interreplenishment intervals per release slots. To get the measures per slot in $2 \mathrm{D}$, we multiplied all the intervals by 10 , assuming 10 slots in total in the $500 \mathrm{~nm}$ circumference cylinder $(50 \mathrm{~nm}$ per a $40 \mathrm{~nm}$ diameter vesicle). In 2D, the distribution is exponential, consistent with a Poisson process. In 1D, a dead time is present, due to the finite diffusion time necessary to access the AZ following the exocytosis of a preceding vesicle.

(c) Replenishment rates per release slot increase exponentially with vesicle packing density in $1 \mathrm{D}$ as well as in 2D (note the logarithmic $y$ axis). Points: vesicle packing density was controlled globally by adding a new vesicle on the top of the simulation space as soon as the packing density decreased below a fixed value. Crosses: a vesicle was added at the top of the simulation volume as soon as no vesicle was present in a band of height $B(40,20,10 \mathrm{~nm}$ in $1 \mathrm{D}$ and $20,10,5 \mathrm{~nm}$ in 2D) at the top of the simulation volume, and reported the replenishment rates as a function of the resulting mean vesicle density. The virtual overlap of these two methods of controlling vesicle density indicates that the global behavior of the system is insensitive to the way a mean packing density is achieved.

(d) Mean vesicle top-down velocities increase exponentially with vesicle packing density in 1D as well as in $2 \mathrm{D}$.

in 1D: 20100 exocytosis events, in 2D 5100 events, from which the first 100 were ignored, to have no transient effects on the distribution. The simulation started with an empty simulation space and the vesicle pool was refilled with a maximum rate of $10 \mathrm{kHz}$ to maintain the relevant packing density. 


\subsection{Discussion}

The advantage of ribbon's presence for synaptic transmission in graded membrane potential sensory cells is not fully understood (Matthews and Fuchs, 2010). Does it catch vesicles from the cytosol to accelerate their arrival to the AZ, or does it trap them to hinder their access to the AZ (Jackman et al., 2009)? Does it enable a large number of release sites (Khimich et al., 2005; Buran et al., 2010; Frank et al., 2010) or play a role in priming (Snellman et al., 2011)? More specifically, the motion of vesicles tethered to the ribbon surface is experimentally barely accessible and not well characterized. How does vesicle tethering and sticking to the ribbon influence vesicle movement, in comparison to free diffusion in the cytoplasm? Depending on the property (i.e. viscosity) of the force, which tethers the vesicles to the ribbon, vesicle motion is not necessarily slowed down. An electrostatic force, for example, can restrict the movement of objects to a plane without decelerating them. Here, we showed that regardless of whether vesicles are decelerated on the ribbon surface or not, their crowding plays a crucial role in creating a lateral pressure to push vesicles toward the AZ. Thus the binding energy of vesicles to the ribbon enables a self-organized pressure pushing vesicles down to the membrane, which could permit rapid replenishment of the RRP. We therefore suggest that the ribbon, in addition to maintaining a halo of vesicles at the active zone, might play the role of a "Brownian conveyor trap". This would be crucial especially during strong stimulation and fast replenishment, when a high vesicle concentration near the release sites would be harder to maintain than in a ribbon-less space. The difference of replenishment efficient with and without a ribbon still remains to be investigated quantitatively. Also, it still needs to be understood how this result could apply to synapses with other electron-dense structures at the active zone (e.g., T-bars (Kittel et al., 2006)) and what could be the advantages of the exact ribbon form: for example rather spherical in hair cells or rather ribbon-line in photoreceptors. 


\subsection{Methods}

To examine vesicle replenishment, we simulated the behavior of vesicles on the synaptic ribbon surface at the active zone (AZ). This incorporated their Brownian motion in a defined one-dimensional (1D) or two-dimensional (2D) geometry, their exocytosis and replenishment. For diffusion we used a Langevin equation approach. It included stochastic forces driving diffusion and deterministic forces, which repelled vesicles from each other and from the boundaries. One type of reaction could occur: exocytosis. In addition refilling of the vesicles pool maintained a constant vesicle packing density.

At each simulations time step, the order of processes was the following: replenishment, exocytosis and diffusion.

\subsubsection{Simulation volume, and vesicle pool replenishment}

Simulations volumes represented either a 1D column on the ribbon or the whole 2D ribbon surface (Fig. 3.1a,b). In 1D, vesicle centers moved on a line with a height $H=$ $300 \mathrm{~nm}$. In 2D, vesicle centers moved on the surface of as cylinder with a perimeter $P=$ $500 \mathrm{~nm}$ and height $H=300 \mathrm{~nm}$. The active zone always refers to the bottom of the simulation volume. Simulations were initialized with 0 vesicles in the simulation volume.

A constant vesicle packing density $\varphi$ was set and maintained by adding vesicles to the simulations volume, modeling trapping of vesicles by the ribbon. The vesicle packing density was defined as $\varphi=F / T$, where $T$ is the total simulation volume and $F$ is the volume filled by vesicles. In $1 \mathrm{D}, T=H$, and $F$ is the sum of the diameters of the vesicles present in the simulation, $F=2 \sum_{i=1}^{n} R_{i}$, where $R_{i}$ is the radius of vesicle $i$ and $n$ is the number of vesicles in the simulation volume. In $2 \mathrm{D}, T=H \cdot P$ and $F$ is the sum the surfaces of the maximal vesicle cross-sections in the volume, $F=\sum_{i=1}^{n} \pi \mathrm{R}^{2}{ }_{i}$. As soon as $\varphi$ decreased below a fixed $\varphi_{0}$ (i.e., $\varphi<\varphi_{0}$ ), a new vesicle was added and placed above the simulation volume, with its center $Z=H+20 \mathrm{~nm}$. In 2D, the horizontal coordinate $X$ was chosen randomly among 10 predefined positions. Due to forces at the upper volume boundary, the vesicles were pushed inside the simulation volume. The theoretical maximum packing density in $2 \mathrm{D}$ is $\varphi_{2 D} \approx 90 \%$ (hexagonal packing). 


\subsubsection{Brownian motion of vesicles}

To simulate vesicle movement, we used an "overdamped" Langevin equation, i.e. a first order stochastic differential equation. In this simplified "position" Langevin equation (Allen and Tildesley, 1989), the evolution equation for a vesicle $i$, subject to deterministic and stochastic forces is:

$$
\frac{d \vec{x}_{i}(t)}{d t}=\frac{D_{i}}{k_{B} T}\left(\sum_{j \neq i} \vec{F}\left(\vec{x}_{j}, R_{j}, \vec{x}_{i}, R_{i}\right)+\vec{F}_{B}\left(\vec{x}_{i}, R_{i}\right)\right)+\sqrt{2 D_{i}} \vec{\xi}_{i}(t)
$$

where $\vec{x}_{k}(t)$ is the position of the vesicle $k$ at time $t, R_{k}$ its radius, $D_{k}$ its diffusion coefficient, $\vec{F}\left(\vec{x}_{k}, R_{k}, \vec{x}_{l}, R_{l}\right)$ the force of vesicle $k$ on vesicle $l, \vec{F}_{B}\left(\vec{x}_{k}, R_{k}\right)$ the force acting of vesicle $k$ due to the simulation boundaries, $k_{B}$ is the Boltzmann constant and $T$ the absolute temperature $\left(295 \mathrm{~K}=22{ }^{\circ} \mathrm{C}\right.$ used). $\vec{\xi}_{i}(t)$ is a zero-mean Gaussian white noise satisfying the auto-correlation relation $\left\langle\vec{\xi}_{i}\left(t_{1}\right) \bullet \vec{\xi}_{j}\left(t_{2}\right)>=d \delta\left(t_{1}-t_{2}\right) \delta_{i j}\right.$, where $\delta(t)$ is the Dirac function, $\delta_{i j}$ is the Kronecker delta and $d$ the number of dimensions in the system.

We didn't consider the evolution of the velocities, as we were interested in time scales significantly longer than the time constant of the velocity auto-correlation function decay $\tau_{i}=m_{i} / \zeta_{i} \approx 10^{-11} \mathrm{~s}=0.01 \mathrm{~ns}$, where $\zeta_{i}$ the drag coefficient due to the surrounding medium, $m_{i}$ is the mass of the vesicle (Allen and Tildesley, 1989). The following consideration shows that this approximation is well justified for our system. The Stokes formula for small Reynolds number and spherical objects is:

$$
\zeta_{i}=6 \pi R_{i} \eta
$$

where $\eta$ the viscosity of the medium. We can rewrite $\tau_{i}$ as:

$$
\tau_{i}=m_{i} / \zeta_{i}=\frac{4}{3} \pi R_{i}^{3} \rho / 6 \pi r_{i} \eta=2 R_{i}^{2} \rho / 9 \eta
$$

where $\rho$ is the density of a vesicle, which is well approximated by the density of water ( $\rho$ $=1 \mathrm{~kg} / \mathrm{m}^{3}$ ) (Takamori et al., 2006). For a rough estimation of $\tau$, we took the viscosity of water $\eta=1 \mathrm{mPa} s$ (viscosity of water at $22^{\circ} \mathrm{C} \sim 1 \mathrm{mPa}$, the cytoplasm has higher viscosity and is non-Newtonian). With these values $\tau=2.2 * 10^{-11} \mathrm{~s}$.

We simulated these equations using the Heun algorithm (Paul and Yoon, 1995), also called stochastic $2^{\text {nd }}$ order Runge-Kutta (Honeycutt, 1992) with a time step $\delta t=10$ $\mu \mathrm{s}$. 


\subsubsection{The diffusion coefficient}

The Einstein-Stokes equation gives us the diffusion coefficient for a spherical object of radium $R$ as a function of temperature, viscosity $\eta$ of a medium:

$$
D_{i}=\frac{k_{B} T}{6 \pi R_{i} \eta}
$$

Thus, the predicted diffusion coefficient of a $20 \mathrm{~nm}$ radius vesicle in water is around $10800 \mathrm{~nm}^{2} / \mathrm{ms}$. Due to the higher viscosity of the cytoplasm, its probable nonNewtonian nature and the crowding due to the presence of other macromolecules (LubyPhelps et al., 1987; Luby-Phelps, 2000), the free diffusion coefficient of a $20 \mathrm{~nm}$ radius vesicle is expected to be around $500 \mathrm{~nm}^{2} / \mathrm{ms}$. The impact of ribbon tethering on vesicle mobility is not well understood - depending on the tethering force properties, vesicle motion might or might not be slowed down. We assumed the vesicle diffusion coefficient ( $D=50 \mathrm{~nm}^{2} / \mathrm{ms}$ for a $40 \mathrm{~nm}$ diameter vesicle) 10 times smaller than predicted for the cytoplasm. Measurements of the diffusion of vesicles in ribbon synaptic terminals indicated values from 10 or $30 \mathrm{~nm}^{2} / \mathrm{ms}$ (Holt et al., 2004) to $110 \mathrm{~nm}^{2} / \mathrm{ms}$ (Rea et al., 2004). These apparent diffusion coefficients are smaller than the expected free diffusion coefficient partly because of vesicle packing (Cichocki and Hinsen, 1990; Gaffield et al., 2006).

For a vesicle, the mean square root displacement was $1 \mathrm{~nm}$ in a given direction according to $\delta x^{2}=2 D \delta t$. The total mean square displacement is $\delta x^{2}=2 d D \delta t$, where $\mathrm{d}$ is the dimensionality of the system.

The apparent diffusion coefficient $D_{a p p}$ was measured according to $D_{a p p .}=<\delta x^{2}>$ / $(2 d \delta t)$, where $d$ is the number of dimension of the system, $\delta x$ is the displacement performed over a measurement time $\delta t$ and $\langle$. $>$ is the averaging over displacements, vesicles and simulations.

\subsubsection{Vesicle repulsion forces}

We modeled the elastic repulsive forces between vesicles, so that they do not overlap and, if they do, rapidly repel each other. The force of a vesicle $j$ on a vesicle $i$ was:

$$
\vec{F}\left(\vec{x}_{j}, R_{j}, \vec{x}_{i}, R_{i}\right)=F_{V} \cdot S\left(\left|\vec{x}_{i}-\vec{x}_{j}\right|-R_{i}-R_{j}\right) \cdot \frac{\vec{x}_{i}-\vec{x}_{j}}{\left|\vec{x}_{i}-\vec{x}_{j}\right|}
$$

with $C_{V}=20 \mathrm{pN}$ and where $\vec{x}_{k}$ and $R_{k}$ are the position and the radius of a vesicle $k$, respectively. $S(d)$ is a sigmoid function: 


$$
S(d)=\left\{\begin{array}{cl}
\frac{1}{1+\operatorname{Exp}\left(\frac{d}{\Delta x}\right)} & d \leq 7 \mathrm{~nm} \\
0 & \text { otherwise }
\end{array}\right.
$$

with $\Delta x=1 \mathrm{~nm}$. For a point positioned at the border of a force field $F$, the mean penetration depth due to random diffusion into the force field is given by $k_{B} T / F$. At room temperature and for a force of $20 \mathrm{pN}$, this is only $0.2 \mathrm{~nm}$. Thus for two vesicles diffusing randomly, they will hardly overlap, as already when touching, the force they exert on each other is $F_{V} / 2=10 \mathrm{pN}$. If a diffusing vesicle penetrated by a distance $d$ into the force field, the average time to diffuse out is: $d / v_{0}=d \cdot k_{B} \cdot T /(F \cdot D)$, where $v_{0}$ is the steady state velocity resulting from the force. Thus for two $20 \mathrm{~nm}$ radius vesicles completely overlapping, and for a diffusion coefficient of $50 \mathrm{~nm}^{2} / \mathrm{ms}$, only after $41 \mu \mathrm{s}$ the vesicles will be apart.

In AFM experiments, vesicles were shown to be less stiff during small deformation, with a stiffness of around $0.2 \mathrm{pN} / \mathrm{nm}$ (Awizio et al., 2007). In the linear regime a deformation of $10 \mathrm{~nm}$ would thus produce a force of only $2 \mathrm{pN}$. But for larger deformation the force increased up to $400 \mathrm{pN}$. We chose the force to grow only up to 20 $\mathrm{pN}$ and to achieve numerical stability in the simulations. The forces were independent of the vesicle.

\subsubsection{Boundary repulsion}

We modeled boundary forces so that vesicles stay inside the defined simulation volume. Let 1 be the coordinate perpendicular to the synaptic membrane and $\hat{e}^{1}$ its unitary vector oriented towards the cytoplasm. The boundary force acting on a vesicle $i$ is defined as:

$$
\vec{F}\left(\vec{x}_{i}, R_{i}\right)=F_{B} S\left(\vec{x}_{i}^{1}-R_{i}\right) \hat{e}^{1}-F_{B} S\left(H-\vec{x}_{i}^{1}-R_{i}\right) \hat{e}^{1}
$$

with $H$ being the simulation volume height, $F_{B}=10 \mathrm{pN}$.

\subsubsection{Exocytosis}

The exocytosis reaction:

$$
\text { Vesicle }+ \text { Membrane } \stackrel{\alpha_{\text {Exo }}}{\longrightarrow} \text { Membrane }
$$

could happen when the vesicle membrane was closer than $15 \mathrm{~nm}$ from the active zone surface. When it was the case, a random number was drawn and if it was smaller than the probability $p_{E \times x}=\delta t \alpha_{E x o}$, where $\delta t$ is the simulation time step, exocytosis occurred. For 
the simulation time step used, this probability was always much smaller than 1. After exocytosis, the vesicle was removed from the simulation volume.

\subsubsection{Parameters summary used for simulations}

\begin{tabular}{|c|c|}
\hline Parameter & Value and remarks \\
\hline Simulation volumes & $\begin{array}{l}\text { 1D: height } H=300 \mathrm{~nm} \text {, referred as the } Z \text { direction } \\
\text { 2D: height } H=300 \mathrm{~nm} \text {, referred as the } Z \text { direction, perimeter } P= \\
500 \mathrm{~nm} \text {, referred as the } \mathrm{X} \text { direction. }\end{array}$ \\
\hline Vesicle radius & $20 \mathrm{~nm}$. \\
\hline Diffusion coefficient & $50 \mathrm{~nm}^{2} / \mathrm{ms}$ for a $20 \mathrm{~nm}$ radius vesicles. \\
\hline Time step $\delta t$ & $0.01 \mathrm{~ms}$ \\
\hline $\begin{array}{l}\text { Replenishment of the vesicle } \\
\text { pool }\end{array}$ & $\begin{array}{l}\text { Maximum rate of } 10 \mathrm{kHz} \text {, so that a minimum filling fraction } \varphi_{0} \text { of the } \\
\text { volume is maintained. New vesicles are placed directly above the } \\
\text { simulation volume. The } \mathrm{X} \text { position is chosen randomly among } 10 \\
\text { positions in the } 2 \mathrm{D} \text { case }\end{array}$ \\
\hline
\end{tabular}

Simulations were performed in $\mathrm{C}++$ and analyzed in Mathematica (Wolfram Research). Random number generator from (Press et al., 2007). 


\section{Release at ribbon synapses: multiquantal, uniquantal or subquantal?}

\section{Short summary}

In chapter 2 we studied how spiral ganglion neurons can precisely encode the timing of almost every neurotransmitter release coming from the inner hair cell ribbon synapses. Such an accurate read out was possible thanks to the extremely large EPSC amplitudes (up to 800 pA). The EPSC giant size was explained a decade ago by the simultaneous release of multiple neurotransmitter quanta. Later, multiquantal release was also observed at other ribbon synapses. In this chapter we investigated, by using modeling, the potential mechanisms underlying multiquantal release. In addition, we proposed an alternative interpretation of the electrophysiological data. 


\subsection{Abstract}

The notion of synchronized multiquantal release (MQR) has driven research on ribbon synapses for a decade (Glowatzki and Fuchs, 2002; Singer et al., 2004; Suryanarayanan and Slaughter, 2006; Keen and Hudspeth, 2006; Goutman and Glowatzki, 2007; Neef et al., 2007; Matthews and Sterling, 2008; Li et al., 2009; Andor-Ardó et al., 2010; Grant et al., 2010). Despite numerous experimental studies, it remains however unclear how the release of multiple neurotransmitter quanta is synchronously without presynaptic spiking. Using biophysical modeling, we determined the constraints that must be fulfilled by two alternative candidate mechanisms: (1) $\mathrm{Ca}^{2+}$ coordinated exocytosis of multiple vesicles, (2) vesicle-to-vesicle (homotypic) fusion prior to exocytosis in order to account for MQR. The coordinated exocytosis model could account for MQR only at very high $\mathrm{Ca}^{2+}$ concentrations and in a limited range of $\mathrm{Ca}^{2+}$ channel open times. For the homotypic fusion model, to avoid the emergence of unrealistically large vesicles, homotypic fusion rates had to decrease with vesicle quantal content. Additionally, to account for the absence of large vesicles in EM experiments, exocytosis rates should increase with vesicle quantal content. The difficulties to implement either of these mechanisms led us to question the concept of $\mathrm{MQR}$ at ribbon synapses. Our modeling suggests that uniquantal release with fusion pore flickering is a plausible alternative explanation for the amplitude and kinetic heterogeneity of postsynaptic currents. 


\subsection{Introduction}

At the presynaptic active zone (AZ), $\mathrm{Ca}^{2+}$ ions entering through voltage-dependent $\mathrm{Ca}^{2+}$ channels trigger the fusion of docked vesicles to the plasma membrane. While the classic view holds that vesicles exocytose independently from each other (del Castillo and Katz, 1954), a presynaptic action potential can coordinate the release of multiple vesicles (multiquantal release: MQR) at a single active zone (AZ) (Wadiche and Jahr, 2001). Surprisingly, ribbon synapses (Fig. 4.1a), even at fixed potential membrane potential, seem to also exhibit multiquantal release (MQR) - the release of several neurotransmitter quanta synchronized to a fraction of a millisecond (Matthews and Fuchs, 2010). With $\mathrm{Ca}^{2+}$ nanodomain control of exocytosis (Brandt et al., 2005; Jarsky et al., 2010), it is conceivable that a shared $\mathrm{Ca}^{2+}$ domain around an open $\mathrm{Ca}^{2+}$ channel synchronizes the exocytosis of multiple vesicles (Fig. 4.1b) (Glowatzki and Fuchs, 2002; Singer et al., 2004). Alternatively, homotypic fusion and subsequent exocytosis of compound vesicles (Pickett and Edwardson, 2006; He et al., 2009) might underlie MQR (Fig. 4.1c) (Edmonds et al., 2004; Matthews and Sterling, 2008).

Characterizing the mode of exocytosis at these sensory synapses is crucial to understand their information transmission and coding capabilities. However, it has so far remained challenging if not impossible to experimentally distinguish between these two alternative candidate mechanisms for MQR in ribbon synapses. This is because of (i) the lack of time resolution of imaging techniques such as electron microscopy, which makes it difficult to distinction of exocytic from endocytic compounds organelles (Lenzi et al., 2002; Matthews and Sterling, 2008), (ii) the limited spatiotemporal resolution of functional light microscopical imaging (Zenisek et al., 2000), and (iii) the limitation electrophysiological techniques to discriminate tightly synchronized fusion of multiple vesicles from the fusion of large compounds (Glowatzki and Fuchs, 2002; Neef et al., 2007; Grant et al., 2010). 
a

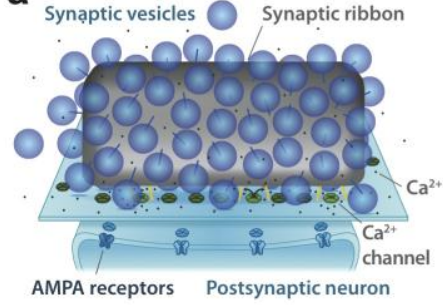

C

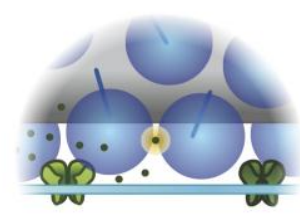

b
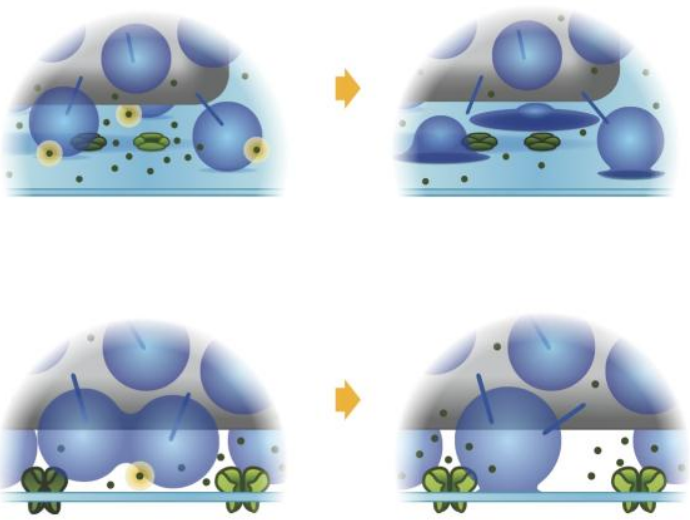

Figure 4.1: Multiquantal release (MQR) at ribbon synapses and its candidate mechanisms.

(a) Scheme of the synaptic connection of a ribbon synapse. Ribbon synapses are highly specialized and transmit sensory information in the ear and the eye with high precision and reliability (Nouvian et al., 2006; Matthews and Fuchs, 2010). They are driven by a graded membrane potential and are distinguished by the presence of a presynaptic electron dense body - the ribbon, to which vesicles tether. The entry of $\mathrm{Ca}^{2+}$ through $\mathrm{Ca}^{2+}$ channels triggers the exocytosis of docked vesicles via activation of the $\mathrm{Ca}^{2+}$ sensor(s) of fusion. The vesicles release their neurotransmitter content into the synaptic cleft. Neurotransmitter molecules bind to the AMPA receptors on the postsynaptic neuron and trigger their opening. This generates excitatory postsynaptic currents (EPSC), which can be recorded by patch-clamp. Channels are not drawn to scale. Open $\mathrm{Ca}^{2+}$ channels are depicted in light green, closed ones are in dark green.

(b) Scheme of $\mathrm{Ca}^{2+}$-coordinated exocytosis. On the left, the opening of a $\mathrm{Ca}^{2+}$ channel (light green) elicits the entry of $\mathrm{Ca}^{2+}$ (green dots) and by binding to the $\mathrm{Ca}^{2+}$ sensor (yellow) triggers the release of 3 nearby docked vesicles. On the right, vesicles fuse with the plasma membrane at slightly different times. Such a multivesicular event would cause an EPSC three times the amplitude of a miniature (uniquantal) EPSC.

(c) Scheme of homotypic fusion of synaptic vesicles. $\mathrm{Ca}^{2+}$ channels open and close stochastically. The homotypic fusion of two vesicles (left) creates a larger compound (middle), which then fuses with the plasma membrane (right). This event would generate an EPSC twice the size of a mini EPSC.

I thank Linda $\mathrm{Hsu}$ for the design of this figure. 
Whereas experimental techniques have been pushed close to their current limits, theoretical approaches were rarely utilized. Here, using biophysical modeling, we examined the fundamental principles and constrains governing (1) coordinated release by a common, pulsed $\mathrm{Ca}^{2+}$ signal and (2) homotypic vesicle fusion dynamics on the synaptic ribbon. We determined the biophysical conditions that need to be satisfied by these two candidate mechanisms for MQR at ribbon synapses can reproduce experimental observations in three systems where MQR has been characterized: the afferent synapses of the rat inner hair cells (IHC) (Glowatzki and Fuchs, 2002; Grant et al., 2010), the afferent synapses of the frog amphibian papilla hair cells (frog $\mathrm{HC}$ ) (Keen and Hudspeth, 2006; Li et al., 2009) and the synapses of the retinal rod bipolar cell (RBC) onto amacrine II cells (Singer et al., 2004; Jarsky et al., 2010).

We modeled $\mathrm{Ca}^{2+}$-coordinated exocytosis of vesicles using quantitative data on $\mathrm{Ca}^{2+}$-dependent exocytosis (Schneggenburger and Neher, 2000; Beutner et al., 2001) and found that $\mathrm{Ca}^{2+}$ channel open times and peak $\mathrm{Ca}^{2+}$ concentration were the critical determinants of release synchronization. MQR could be reproduced only by high peak $\mathrm{Ca}^{2+}$ concentrations and in a limited range of channel open times. Using realistic simulations of vesicles diffusion, exocytosis and homotypic fusion, we observed that homotypic fusion is potentially a self-amplifying process that can create vesicles of unlimited size. $\mathrm{Ca}^{2+}$ dependent homotypic fusion however prevented the creation of extremely large compounds if homotypic fusion rates decreased with the vesicle quantal content. In addition, to account for the absence of large vesicle compounds in EM images, exocytosis rates should in contrast increase with vesicle quantal content.

In view of the difficulty to implement either of the mechanisms biophysically, we considered an alternative hypothesis: that large EPSCs result from a complete release of a vesicle neurotransmitter content, whereas small (usually considered as uniquantal) EPSCs result from short pore flickers. We find that the large EPSCs found in the IHC (mean: $300-400 \mathrm{pA})$ and frog HC (100-200 pA) could potentially result from the activation of a large glutamate receptor cluster by the neurotransmitter content of a single vesicle. Small EPSCs could result from a short fusion pore flicker and fusion pore dynamics could account for the heterogeneity of EPSC waveforms. While our study does not rule out any of the scenarios, it reveals important constraints for the currently considered scenarios of MQR and proposes a hitherto not considered explanation of MQR: uniquantal and subquantal release. In addition our study indicates directions for future experiments. 


\begin{tabular}{|c|c|c|c|}
\hline System & $\begin{array}{l}\text { Mature rat IHC } \\
\text { (monophasics EPSCs) } \\
\text { (Glowatzki and Fuchs, 2002; } \\
\text { Grant et al., 2010) }\end{array}$ & $\begin{array}{l}\text { Amphibian papillae } \\
\text { HC of adult } \\
\text { bullfrogs (Li et al., } \\
2009)\end{array}$ & $\begin{array}{l}\text { Rod bipolar cell to } \boldsymbol{A I I} \\
\text { amacrine cells (Singer et } \\
\text { al., 2004; Jarsky et al., } \\
2010 \text { ) }\end{array}$ \\
\hline $\begin{array}{l}\text { Average } 10-90 \% \text { rise time of } \\
\text { multiquantal EPSCs }\end{array}$ & 0.14 ms (Grant et al., 2009) & $0.25 \mathrm{~ms}$ & $\begin{array}{l}0.3 \mathrm{~ms} \text { (from, } 95 \% \text { of } \\
\text { EPSCs) }\end{array}$ \\
\hline $\begin{array}{l}\text { Largest event size (in terms of } \\
\text { number of quanta), } N_{A}\end{array}$ & $\begin{array}{l}16(800 \mathrm{pA} \text { with } 50 \mathrm{pA} \\
\text { unitary size) }\end{array}$ & $\begin{array}{l}7(350 \mathrm{pA} \text { with a } \\
\text { unitary size of } 50 \mathrm{pA})\end{array}$ & $\begin{array}{l}2 \text { (as reported by the } \\
\text { binomial dist. with } \mathrm{n}=2 \text { ) }\end{array}$ \\
\hline $\begin{array}{l}\text { Mean quanta number released } \\
\text { per event, } N_{R}\end{array}$ & $\begin{array}{l}7.5 \text { (375 pA as mean in Fig. } \\
\text { 4G of (Grant et al., 2010)) }\end{array}$ & $\begin{array}{l}2.7(137 \mathrm{pA} \text { as mean } \\
\text { in Fig. 1B) }\end{array}$ & $\begin{array}{l}1.64 \text { (given as } m \text { in Fig. 6a } \\
\text { in (Singer et al., 2004)) }\end{array}$ \\
\hline Mean release probability $P_{R}$ & 0.47 & 0.37 & 0.78 \\
\hline $\begin{array}{l}\text { Coefficient of variation }(C V) \\
\text { of the quantal content } \\
\text { distribution }\end{array}$ & \multicolumn{3}{|c|}{$0.16<C V<0.32$ (Supplementary Fig. 4.3) } \\
\hline $\begin{array}{l}\text { Skewness of the quantal } \\
\text { content distribution }\end{array}$ & \multicolumn{3}{|c|}{$-0.8<$ Skewness $<0.2$ (Supplementary Fig. 4.3) } \\
\hline
\end{tabular}

Table 4.1: Critical parameters characterizing synchronized MQR in three systems.

The three systems we considered are the afferent synapses of the rat inner hair cells (IHC) (Glowatzki and Fuchs, 2002; Grant et al., 2010), the afferent synapses of the frog amphibian papilla hair cells (frog HC) (Keen and Hudspeth, 2006; Li et al., 2009) and the synapses of the retinal rod bipolar cell (RBC) onto amacrine II cells (Singer et al., 2004; Jarsky et al., 2010). In the rat mature IHC, EPSC kinetics were highly variable, ranging from fast monophasic to slow multiphasic EPSCs, we considered the monophasic EPSCs, as they are the most frequent in mature animals (Grant et al., 2010) and pose the most stringent criteria for synchronization. Average reported values of the critical parameters for MQR. The number of available vesicle $N_{A}$ during an isolated $\mathrm{Ca}^{2+}$ pulse was taken as the ratio of the largest observed EPSC amplitude to the miniature EPSC (mEPSC) amplitude. The mean release probability $P_{R}$ was calculated using binomial statistics neglecting the null events and with the parameters $N_{R}$ and $N_{A}$, by solving equation (4.4). 
a

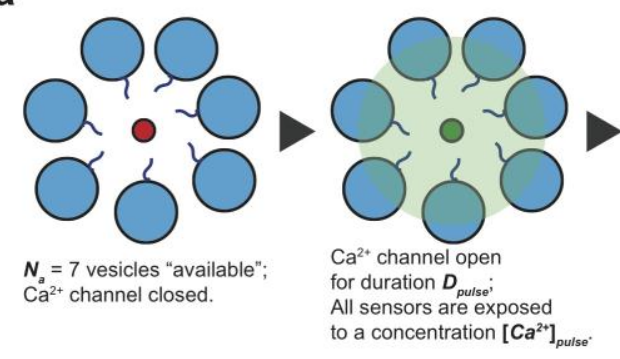

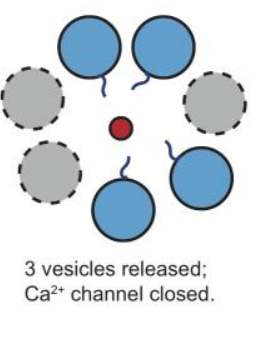

e

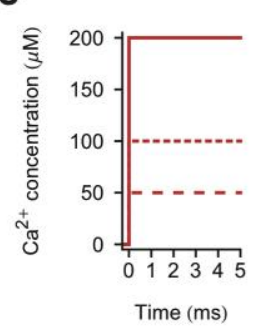

g

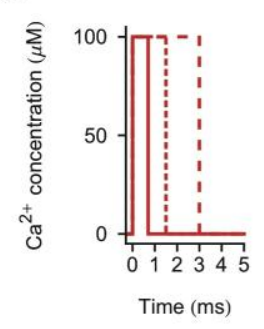

d

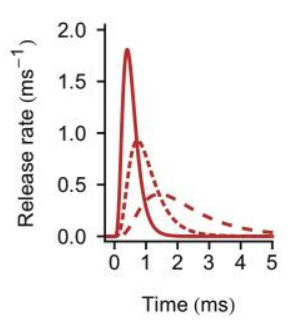

h

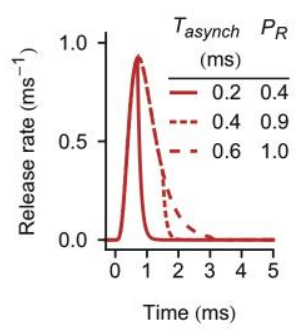

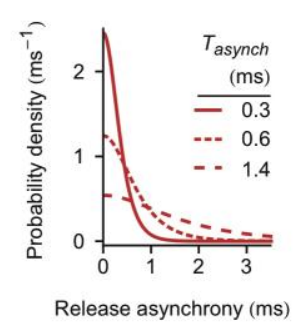

i

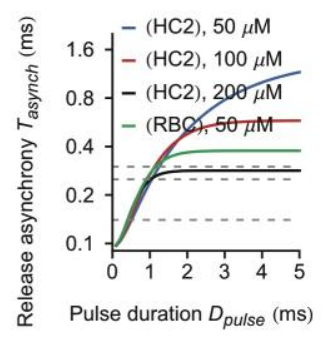

b

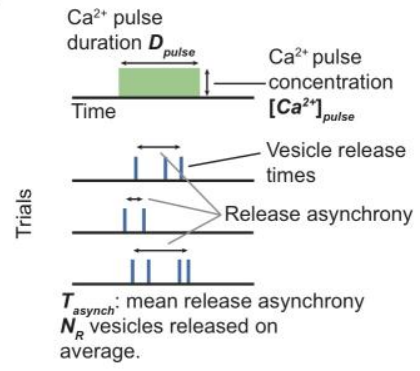

f

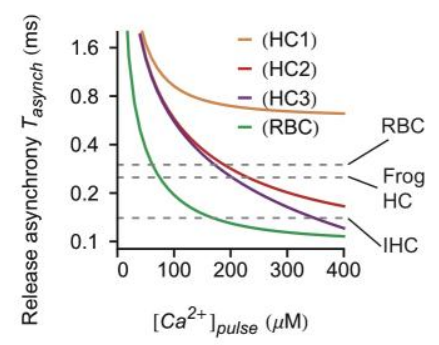

j

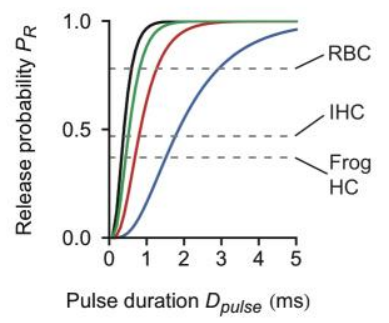

Figure 4.2: $\mathrm{Ca}^{2+}$ pulse concentration and duration determine the synchrony of vesicle release in $\mathrm{Ca}^{2+}$-coordinated exocytosis.

(a) $N_{A}=7$ vesicles are located near a closed $\mathrm{Ca}^{2+}$ channel (red). The $\mathrm{Ca}^{2+}$ channel opens (green) for a duration $D_{\text {pulse }}$ and $\mathrm{Ca}^{2+}$ sensors located on the vesicles sense a $\mathrm{Ca}^{2+}$ concentration $\left[\mathrm{Ca}^{2+}\right]_{\text {pulse }}$. In this example, $\mathrm{Ca}^{2+}$ channel opening caused 3 vesicles to fuse and release their neurotransmitter content.

(b) If the same pulse is repeated multiple times, the release asynchrony and the number of vesicles released vary.

(c)-(e) Increasing the $\mathrm{Ca}^{2+}$ pulse concentration (c), increases the maximum release rate and decreases the release mean latency and jitter (d). As a result it decreases the release asynchrony $T_{\text {asynch }}(\mathrm{e})$. The most probable release time lag is $0 \mathrm{~ms}$. Here using (Beutner et al., 2001) models with $\gamma=10 \mathrm{~ms}^{-1}$.

(f) Decrease of release asynchrony $T_{\text {asynch }}$ with increasing $\mathrm{Ca}^{2+}$ concentration for different release models. ( $\mathrm{HC1}-\mathrm{HC} 3$ ) are the (Beutner et al., 2001) models with the final release rate $\gamma=$ $1.7 \mathrm{~ms}^{-1}, 10 \mathrm{~ms}^{-1}, 100 \mathrm{~ms}^{-1}$, respectively. ( $\mathrm{HC1}$ ) is the $\mathrm{Ca}^{2+}$ sensor found experimentally in (Beutner et al., 2001). (HC2) is used in this work for the IHC and the frog HC. (RBC) is the model of (Schneggenburger and Neher, 2000) with $\gamma=10 \mathrm{~ms}^{-1}$, used for the RBC. The gray dashed horizontal lines are the 10-90\% EPSC rise times for the three systems considered (Table 4.1). The intersections of the gray horizontal dashed lines with the release time lag curves indicate the minimum $\mathrm{Ca}^{2+}$ concentration $\left[\mathrm{Ca}^{2+}\right]_{\text {pulse }}$ able to reproduce the experimentally found release synchrony in each system.

(g)-(j) Decreasing the $\mathrm{Ca}^{2+}$ pulse duration $D_{\text {pulse }}(\mathrm{g})$ shortens the duration of release (h) and thus decreases the release asynchrony $T_{\text {asynch }}$ (i) and the release probability $P_{R}$ (j). (i) and (j): $T_{\text {asynch }}$ and $P_{R}$ as a function of $D_{\text {pulse }}$ for 3 different $\mathrm{Ca}^{2+}$ concentrations for the (HC2) sensor and one $\mathrm{Ca}^{2+}$ concentration for the (RBC) sensor. Gray horizontal dashed lines in (i) are as in (f). Gray horizontal dashed lines in (j) represent the release probabilities in each of the three synapses (Table 4.1). The intersections of the gray horizontal dashed lines to the release 
probability curves reveal the minimum $D_{\text {pulse }}$ required to reproduce the experimental mean number of released vesicles $N_{R}$ per EPSC. Release model as in (f).

\subsection{Results}

\subsubsection{Coordination of vesicle release by $\mathrm{Ca}^{2+}$ nanodomain control of exocytosis}

Ribbon synapses exhibit synchronized multiquantal release (MQR), the simultaneous release of multiple neurotransmitter quanta without a presynaptic spike (Matthews and Fuchs, 2010). At the same time, exocytosis in ribbon synapses is likely governed by $\mathrm{Ca}^{2+}$ nanodomains (Brandt et al., 2005; Moser et al., 2006; Goutman and Glowatzki, 2007; Jarsky et al., 2010), where the opening of one or few $\mathrm{Ca}^{2+}$ channels can trigger the release of a vesicle. Here we examined whether coordinated fusion in response to temporally isolated $\mathrm{Ca}^{2+}$ pulses generated by the opening of a single $\mathrm{Ca}^{2+}$ channel could be the mechanism underlying MQR for the IHC, for HC and RBC (Table 4.1).

To investigate the key factors governing synchronization of vesicle exocytosis, we first studied to what extend a long $(10 \mathrm{~ms})$, temporally isolated, $\mathrm{Ca}^{2+}$ pulse of concentration $\left[\mathrm{Ca}^{2+}\right]$ puse can coordinate vesicle release (Fig. 4.2a-f, Methods). Using established $\mathrm{Ca}^{2+}$-dependent secretion models (Schneggenburger and Neher, 2000; Beutner et al., 2001) we calculated the resulting vesicle release rates. Increasing $\left[\mathrm{Ca}^{2+}\right]$ pulse led to larger maximum release rates, to a smaller average release latency and jitter and as a consequence to an smaller release asynchrony $T_{\text {ayynch }}$ (Fig. 4.2c-f). $T_{\text {arynch }}$ first rapidly decreased and then saturated for higher $\left[\mathrm{Ca}^{2+}\right]_{\text {pulse }}$. The smallest possible $T_{\text {a.synch }}$ is determined by the $\mathrm{Ca}^{2+}$-independent rate of the final step of the exocytosis process. This release jitter is intrinsic to the release process itself and cannot be overcome even by further increasing $\left[\mathrm{Ca}^{2+}\right]$ pulse. Thus the IHC secretion scheme, here applied to both HC systems (Beutner et al., 2001) could only synchronize vesicle down to $0.6 \mathrm{~ms}$ (Fig. 4.2f, (HC1)), due to the slow $\left(\gamma=1.7 \mathrm{~ms}^{-1}\right) \mathrm{Ca}^{2+}$-independent last release step. Augmenting this last limiting fusion rate $\gamma$ to $10 \mathrm{~ms}^{-1}$ (Millar et al., 2005) (used in the rest of the work) and $100 \mathrm{~ms}^{-1}$ highly enhanced its synchronization capability (Fig. 4.2f, (HC2) and (HC3)). The release scheme for the calyx of Held ((Schneggenburger and Neher, 2000) with $\gamma$ set to $10 \mathrm{~ms}^{-1}$ ) exhibited a higher sensitivity to $\mathrm{Ca}^{2+}$ than observed in hair cells and was assumed for the RBC (Fig. 4.2f, (RBC)). 
For the $\mathrm{RBC},\left[\mathrm{Ca}^{2+}\right]_{p u l s e}>60 \mu \mathrm{M}$ was required to reproduce the synchrony of the $10-90 \%$ rise time of a multiquantal EPSC. For the frog $\mathrm{HC}$ and the IHC even higher concentrations (above $200 \mu \mathrm{M}$ and $400 \mu \mathrm{M}$, respectively) were required to match the experimentally observed synchrony. Even if the ribbon plays the role of a reflecting surface increasing $\mathrm{Ca}^{2+}$ concentration below it, the concentration resulting from the opening of a single $\mathrm{Ca}^{2+}$ channel might still be too low to synchronized the fusion of multiple vesicles (Roberts, 1994). Thus, long $\mathrm{Ca}^{2+}$ pulses of realistic concentration appeared insufficient to account for MQR.

Shortening the duration $D_{\text {pulse }}$ of the $\mathrm{Ca}^{2+}$ pulse, however, constrained vesicles exocytosis times essentially to the interval of the pulse and as a result could decrease the release asynchrony down to $0.1 \mathrm{~ms}(\approx 1 / \gamma$, Fig. 4.2 g-i $)$, which is smaller than the EPSC rise time in any of the three systems. But this also resulted in decreasing the vesicle release probability $P_{R}$, which could impede MQR (Fig. 4.2j, Table 4.1).

\subsubsection{Simulation of $\mathrm{Ca}^{2+}$ coordinated exocytosis suggest a trade of efficiency for temporal precision}

The low release asynchrony $T_{a s y n c h}$ and high release probability $P_{R}$ impose opposing requirements on the $\mathrm{Ca}^{2+}$ pulse duration $D_{\text {pulse }}$ in order for the coordinated fusion model to reproduce the experimental observations of MQR (Supplementary Fig. 4.2). Moreover, as the $\mathrm{Ca}^{2+}$ pulses originate from the opening of a $\mathrm{Ca}^{2+}$ channel, $D_{\text {pulse }}$ is expected to be exponentially distributed: $\operatorname{Prob}\left(D_{\text {pulse }}\right) \propto \exp \left(-D_{\text {pulse }} / \tau_{\text {open }}\right)$, where $\tau_{\text {open }}$ is the mean $\mathrm{Ca}^{2+}$ channel open time. Therefore we systematically studied for which combinations of channel mean open time $\tau_{\text {open }}$ and concentration $\left[\mathrm{Ca}^{2+}\right]_{p u l s e}$ this model is consistent with experimental observation (called consistency region in the parameter space) in terms of the mean number of vesicles released per event $\overline{N_{R}}$ and the mean release asynchrony $\overline{T_{\text {asynch }}}$ (Methods). We calculated $\overline{N_{R}}$ and $\overline{T_{a \text { asynch }}}$ for ranges of $\left[\mathrm{Ca}^{2+}\right]_{\text {pulse }}$ (up to $250 \mu \mathrm{M})$ and $\tau_{\text {open }}\left(0\right.$ to $2 \mathrm{~ms}$ ). A $\left[\mathrm{Ca}^{2+}\right]_{\text {pulse }}$ of $200 \mu \mathrm{M}$ is reached at about $18 \mathrm{~nm}$ of a $\mathrm{Ca}^{2+}$ channel center, assuming mild conditions of $\mathrm{Ca}^{2+}$ buffering ( $1 \mathrm{mM}$ EGTA) and single channel $\mathrm{Ca}^{2+}$ current of $1 \mathrm{pA}$ (calculated using (Naraghi and Neher, 1997)). Closer distances, and as a result higher $\left[\mathrm{Ca}^{2+}\right]_{\text {pulse }}$ seem implausible when more than 3 vesicles of $20 \mathrm{~nm}$ radius need to be positioned in the proximity of a $\mathrm{Ca}^{2+}$ channel. We explored the range of mean open times $\tau_{\text {open }}$ reported for Cav1.3 L-type $\mathrm{Ca}^{2+}$ channels (RodriguezContreras and Yamoah, 2001; Zampini et al., 2010). 
$\mathrm{Ca}^{2+}$-triggered coordinated exocytosis model of MQR

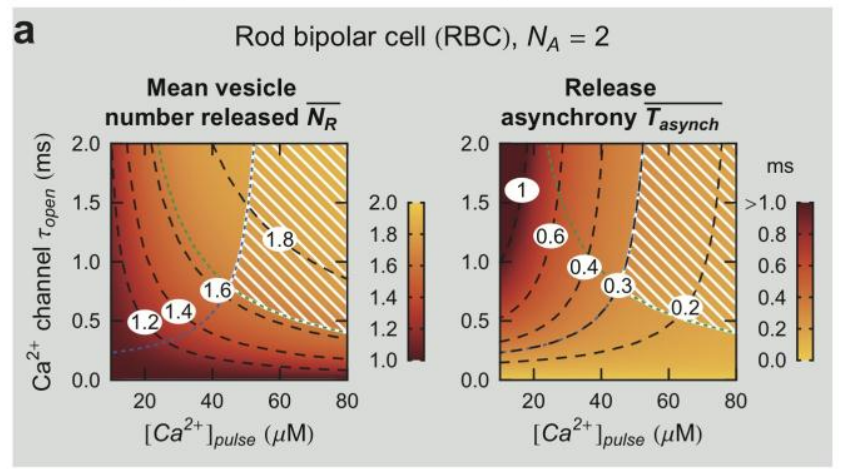

b Bullfrog amphibian papilla hair cell (frog $\mathrm{HC}$ ), $N_{A}=7$
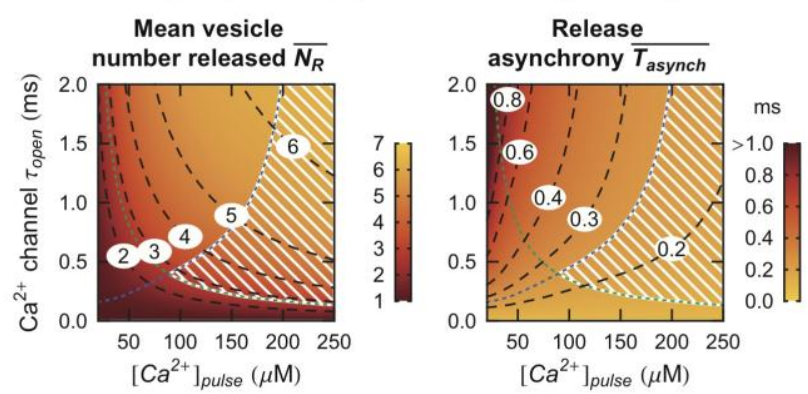

C Rat inner hair cell (IHC), monophasic EPSCs, $N_{A}=16$
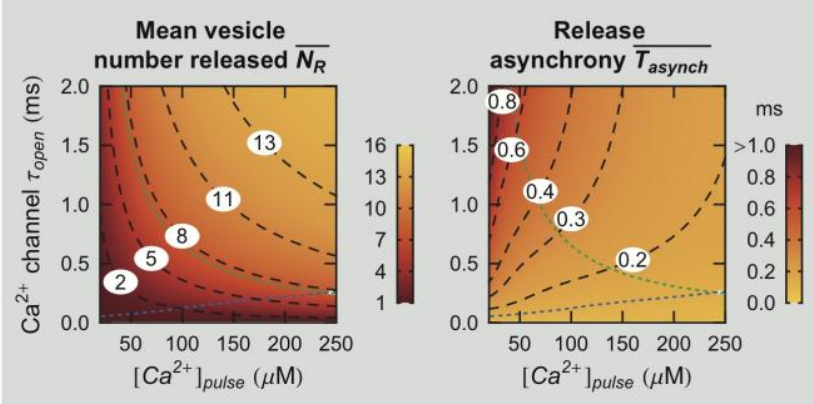

Figure 4.3: Screening the temporal precision and efficiency of $\mathrm{Ca}^{2+}$ coordinated release model of $M Q R$ in terms of $\left[\mathrm{Ca}^{2+}\right]_{\text {pulse }}$ and $\tau_{\text {Open }}$.

Mean number of vesicles released $\overline{N_{R}}$ and the release asynchrony $\overline{T_{\text {asynch }}}$ as a function of $\mathrm{Ca}^{2+}$ pulse concentration $\left[\mathrm{Ca}^{2+}\right]_{\text {pulse }}$ and $\mathrm{Ca}^{2+}$ channel mean open time $\tau_{\text {Open }}$. Each row represents the coordinated exocytosis model applied to a specific system, assuming a particular number of available vesicles $N_{A}$ during a $\mathrm{Ca}^{2}$ pulse (Table 4.1). The consistency region (white stripes) is where the model is consistent with experimental observation (Table 4.1). The blue and green dashed lines result from the criteria on the synchrony and the mean number of vesicles released, respectively.

(a) The model can reproduce experimental observations for $\left[\mathrm{Ca}^{2+}\right]_{\text {pulse }}$ $>40 \mu \mathrm{M}$ and $\tau_{\text {Open }}>0.5 \mathrm{~ms}$. Using (Schneggenburger and Neher, 2000) secretion model with final release step $\gamma$ $=10 \mathrm{~ms}^{-1}$.

(b) The consistency region is at $\left[\mathrm{Ca}^{2+}\right]_{\text {pulse }}>70 \mu \mathrm{M}$ and $\tau_{\text {Open }}$ around 0.5 ms. Using (Beutner et al., 2001) secretion model with $\gamma=10 \mathrm{~ms}^{-1}$.

(c) The consistency region is beyond $250 \mu \mathrm{M}$. Using (Beutner et al., 2001) secretion model with $\gamma=10 \mathrm{~ms}^{-1}$. 
We found that the mean number of vesicles released $\overline{N_{R}}$ increased with $\left[\mathrm{Ca}^{2+}\right]$ pulse and $\tau_{\text {open }}$ (Fig. 4.3, first column). The region where $\overline{N_{R}} \approx N_{A}$ (the number of vesicles feeling the $\mathrm{Ca}^{2+}$ pulse) corresponds to where the probability of release $P_{\mathrm{R}} \approx 1$. In this area the release asynchrony $\overline{T_{\text {asynch }}}$ mainly decreased with increasing $\left[\mathrm{Ca}^{2+}\right]_{\text {pulse }}$ (Fig. 4.3, second column). In the region where $P_{R}<1, \overline{T_{\text {asyncb }}}$ mainly decreased with $D_{\text {pulse. }}$ The lower bound (dotted green line) of the region results from the requirements on the minimum number of vesicles released during a $\mathrm{Ca}^{2+}$ pulse. The upper bound (dotted blue line) results from the requirements on the release synchrony. We found that the consistency region (Table 4.1, white striped area) differs considerably for the three systems, suggesting that the coordinated release mechanism is not equally plausible for each of them.

For the RBC, the consistency region was extended (Fig. 4.3a), it started from $\left[\mathrm{Ca}^{2+}\right]_{\text {Pulse }}>50 \mu \mathrm{M}$ and $\tau_{\text {open }}>0.5 \mathrm{~ms}$. This parameter region is plausible during $\mathrm{RBC}$ depolarization. MQR however was also reported to occur hundreds of ms after synapse depolarization at low buffer conditions (Singer et al., 2004), when no ion channel flicker is expected and when the global $\mathrm{Ca}^{2+}$ is likely in the low or sub- $\mu \mathrm{M}$ range. While synchronization by local $\left[\mathrm{Ca}^{2+}\right]$ transients cannot be excluded, this observation argues against the coordination of release by a common $\mathrm{Ca}^{2+}$ channel as a mechanism of $\mathrm{MQR}$ in RBC.

For the frog HC, the model could reproduce experimental observations only for much higher $\left[\mathrm{Ca}^{2+}\right]_{\text {pulse }}$ (Fig. 4.3b). The consistency region encompasses values of $\left[\mathrm{Ca}^{2+}\right]_{\text {pulse }}$ above $100 \mu \mathrm{M}$ and $\tau_{\text {open }}$ around $0.4 \mathrm{~ms}$. For higher $\left[\mathrm{Ca}^{2+}\right]_{\text {pulse }}$ the permissive $\tau_{\text {open }}$ values extended from $0.2 \mathrm{~ms}$ to beyond $2 \mathrm{~ms}$. This parameter range could match the accessible experimental conditions, when MQR is observed. If this is the mechanism underlying MQR in frog $\mathrm{HC}$, we expect that application of BayK should desynchronize vesicle release, as it increases the average channel open time. Also homogeneous elevation of $\left[\mathrm{Ca}^{2+}\right]$ in the hair cell (e.g. by $\mathrm{Ca}^{2+}$ uncaging) should reveal EPSC desynchronization.

For the IHC, the model did not predict sufficient synchronization of release to account for the occurrence of monophasic EPSCs within a plausible parameter range (Fig. 4.3c). For the synchrony and the mean number of released quanta to be large enough, $\left[\mathrm{Ca}^{2+}\right]_{\text {pulse }}$ needed to exceed $250 \mu \mathrm{M}$. Such concentrations seem unrealistic for the IHC, considering that up to 16 vesicles should sense this concentration simultaneously 
(Grant et al., 2010). Coordinated release could however be a mechanism for multiphasic EPSCs.

That $\mathrm{Ca}^{2+}$ coordinated exocytosis does not appear as a plausible mechanism of MQR common to different ribbon synapses is also suggested by the fact that MQR was observed during strong synapse stimulation and $\mathrm{Ca}^{2+}$ influx (Singer et al., 2004; Keen and Hudspeth, 2006; Goutman and Glowatzki, 2007; Li et al., 2009). First, in this regime $\mathrm{Ca}^{2+}$ domains coming from different $\mathrm{Ca}^{2+}$ channels would not be temporally isolated and would spatially overlap, thus failing to provide a synchronizing $\mathrm{Ca}^{2+}$ signal to a pool of ready releasable vesicles. Second, replenishment is then the rate-limiting step of the vesicle cycle, and MQR would not only require coordinated exocytosis, but also coordinated physical arrival to the active zone (AZ), coordinated docking and coordinated priming, all with a jitter $200 \mu \mathrm{m}$. This seems highly implausible provided that all steps of the vesicle cycle are slower than exocytosis and thus produce even more jitter (Pan and Zucker, 2009). For all of these reasons, coordinated release is an unlikely mechanism of MQR. Which mechanism could underlie MQR during constant vesicle replenishment and $\mathrm{Ca}^{2+}$ influx? We next considered homotypic vesicle fusion as a potential mechanism for MQR in ribbon synapses. 
a
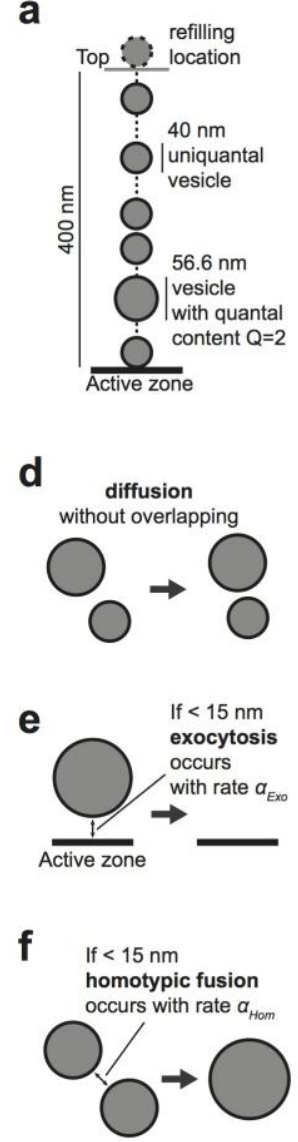

b

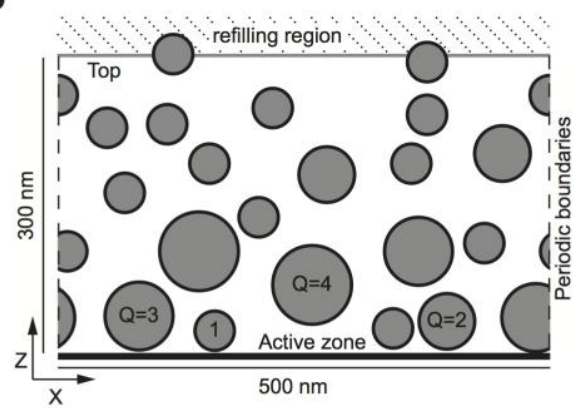

i

g
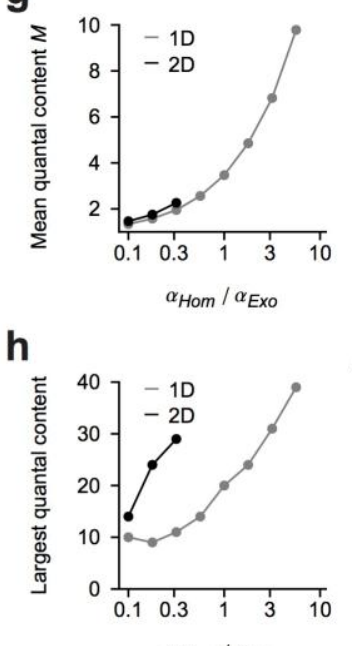

$\alpha_{\text {Hom }} / \alpha_{\text {Exo }}$
C

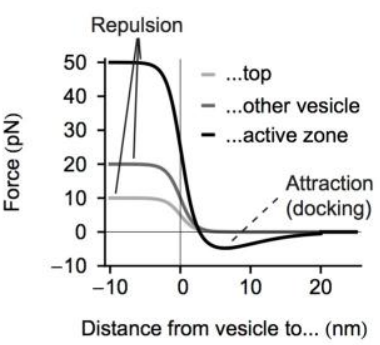

k

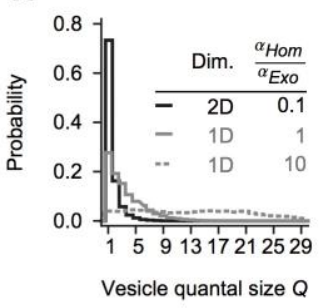

Figure 4.4: Homotypic fusion model for $M Q R$

(a) Scheme of the one-dimensional simulation column. The centers of the vesicles moved on a vertical line.

(b) Scheme of the two-dimensional simulation cylinder. The centers of the vesicles moved on the cylinder surface. $Q$ represents the quantal content of vesicles.

(c) Forces present in the simulation. The active zone attracted vesicles that were in close proximity, but did not let them cross the plasma membrane. Vesicles repulsed each other with a maximal force of $20 \mathrm{pN}$. The top of the simulation pushed the vesicles into the simulation volume with a maximal force of $10 \mathrm{pN}$.

(d) Vesicles diffused without overlapping with a diffusion coefficient $\left(D=50 \mathrm{~nm}^{2} / \mathrm{ms}\right.$ for a 40 $\mathrm{nm}$ diameter vesicle). The diffusion coefficient of vesicles decreased with size according to the Einstein-Stokes formula (Methods).

(e) Vesicles exocytosed with a rate $\alpha_{E x o}=3 \mathrm{~Hz}$ when closer than $15 \mathrm{~nm}$ to the active zone (AZ). Exocytosis resulted in vesicle disappearance from the simulation volume.

(f) Homotypic fusion between two vesicles happened with a rate $\alpha_{\text {Hom }}$ when they were closer than $15 \mathrm{~nm}$ from each other. The two parent vesicles disappeared and the newly created spherical vesicle emerged in the center of mass of the two parent vesicles. The quantal content (i.e. neurotransmitter content) and total membrane surface were summed.

(g)-(j) Critical characteristics of the exocytic quantal content distributions in $1 \mathrm{D}$ and 2D simulations as a function of $\alpha_{H o m} / \alpha_{E x o}$. Note the logarithmic scale in $\mathrm{x}$. Points with maximum $\alpha_{H o m} / \alpha_{E x o}$ are the last simulations where vesicles quantal content $Q$ was smaller than 40 . Gray horizontal dashed lines delimit the range of experimental distributions (Table 4.1). Quantal content distributions became wider and extremely large vesicles were created for larger $\alpha_{H o m} / \alpha_{\text {Exo }}$. Distributions as observed in the experiments could not be reproduced.

(k) Quantal content distributions for three values of $\alpha_{H o m} / \alpha_{\text {exo }}$ in 1D and 2D. The simulation in 1D with $\alpha_{\text {Hom }} / \alpha_{\text {exo }}=10$ had vesicles with $\mathrm{Q}>40$. Shown is the histogram of the exocytosed events before the $Q=40$ vesicle was created. 


\subsubsection{Homotypic fusion model of MQR}

MQR might result from homotypic fusion of vesicles prior to exocytosis (Edmonds et al., 2004; Matthews and Sterling, 2008). Compound vesicles composed of a variable number of elementary neurotransmitter quanta would then constitute the readily releasable pool. To derive the predicted quantitative features of MQR in the homotypic fusion scenario, we modeled the homotypic fusion and exocytosis of vesicles during their Brownian motion on the synaptic ribbon surface (Fig. 4.4a-f, Methods). The vesicle population present on the ribbon seemed most relevant to the homotypic fusion scenario as vesicles are thoughts to access the $A Z$ release sites mainly via movement on the ribbon (Zenisek et al., 2000; Holt et al., 2004; LoGiudice and Matthews, 2009). In the first model, vesicles diffused vertically in a one-dimensional column of $400 \mathrm{~nm}$ high, perpendicular to the AZ (Fig. 4.4a, 1D model). This model would apply if vesicle motion was restricted to vertical tracks on the ribbon surface. In a second model, vesicle moved on a two-dimensional cylindrical surface of $300 \mathrm{~nm}$ high and $500 \mathrm{~nm}$ circumference, representing the entire ribbon surface (Fig. 4.4b, 2D model) (Nouvian et al., 2006). Forces at the top and bottom boundaries constrained vesicles to stay inside the simulation volume.

Physical docking of vesicles was mimicked by irreversible attachment to the AZ at the bottom of the simulation volume (Fig. 4.4c). Vesicles within $15 \mathrm{~nm}$ of the AZ, fused with the plasma membrane at a fixed rate $\left(\alpha_{E x o}=3 \mathrm{~Hz}\right.$ per vesicle). This slow $\alpha_{E \times 0}$ represents the effective rate of molecular docking, priming and exocytosis and was the rate-limiting step of the vesicle cycle. In $2 \mathrm{D}$ it yielded a total maximum release rate of about $36 \mathrm{~Hz}$ and allowed time for vesicles to perform homotypic fusion before exocytosis. To model the capturing of cytosolic vesicles by the ribbon, vesicles were replenished at the top of the volume, maintaining a constant vesicle packing density $\left(\varphi_{1 D}\right.$ $=70 \%, \varphi_{2 D}=40 \%$ ), corresponding to a steady state condition of release (Lenzi et al., 2002). When two vesicles approached were closer than $15 \mathrm{~nm}$, they became capable of homotypic fusion with a rate $\alpha_{H o m}$. As they fused, their neurotransmitter content and membrane surfaces were summed and a daughter vesicle of spherical shape emerged in the center of mass of the two parent vesicles. 


\subsection{4 $\mathrm{Ca}^{2+}$ independent homotypic fusion}

First we assumed homotypic fusion rates were $\mathrm{Ca}^{2+}$-independent: vesicles fused homotypically with the same rate $\alpha_{\text {Hom }}$ everywhere on the ribbon surface. This could apply to the IHC, where the EPSC distributions is invariant for different $\mathrm{Ca}^{2+}$ buffering conditions (Goutman and Glowatzki, 2007). We found that the ratio of homotypic fusion rate to exocytosis rate $\alpha_{H o m} / \alpha_{E x o}$ was the most important parameter determining the shape of the exocytic quantal content distribution (Fig. $4.4 \mathbf{g}-\mathbf{j})$. For small $\alpha_{\text {Hom }} / \alpha_{\text {Exo }}$ the distribution was strongly positively skewed with the highest proportion of uniquantal vesicles. As the ratio was increased, extremely large vesicles (quantal content $Q>40$ ) were created due to runaway homotypic fusion. In contrast to the predicted distributions by the model, in all systems examined (RBC, Frog HC and IHC) these distributions have been reported to be relatively narrow and symmetric, as quantified by their coefficients of variation $C V$ and skewnesses (Table 4.1, Supplementary Fig. 4.3). This model of homotypic fusion could thus not reproduce the experimental distributions, potentially because homotypic fusion could occur everywhere in the simulation space.

\subsection{5 $\mathrm{Ca}^{2+}$ regulated homotypic fusion}

Next we studied $\mathrm{Ca}^{2+}$ regulated homotypic fusion, assuming homotypic fusion rates were depended on local $\left[\mathrm{Ca}^{2+}\right]$ (He et al., 2009). Because $\left[\mathrm{Ca}^{2+}\right]$ is known to decay with the distance from the AZ (Roberts, 1993; 1994; Naraghi and Neher, 1997; Frank et al., 2009), we let the homotypic fusion rate $\alpha_{H o m}$ decrease with the distance $z$ from the AZ to the site of vesicle interaction, effectively constraining homotypic fusion to AZ proximity (Fig. 4.5a,b, Supplementary note):

$$
\alpha_{\text {Hom }}(z)=H_{\text {Max }} e^{-\left(\frac{z-z_{0}}{\lambda_{z}}\right)^{2}} \theta\left(z-z_{0}\right)
$$

where $H_{\text {Max }}$ is the maximum homotypic rate. $\lambda_{z}$ is the characteristic decay length from the $\mathrm{AZ}$ of the homotypic fusion rate, caused by the $\left[\mathrm{Ca}^{2+}\right]$ decay. $z 0$, the height where the homotypic fusion rate equals $H_{\text {Max }}$, was set to $40 \mathrm{~nm}$ (a uniquantal vesicle diameter) and to $20 \mathrm{~nm}$ (a uniquantal vesicle radius) in $1 \mathrm{D}$ and in $2 \mathrm{D}$, respectively. $z_{0}$ corresponded to the lowest possible interaction point between two vesicles. $\theta(z)$ is the Heaviside step function. Experimentally, $H_{M a x}$ and $\lambda z$ could be manipulated by changing the $\left[\mathrm{Ca}^{2+}\right]$ profile at the AZ. Note that $H_{M a x}$ and the rate of vesicle fusion to the plasma membrane $\alpha_{\text {Exo }}$ might have different dependences on $\mathrm{Ca}^{2+}$ concentration. To screen a large parameter space, we varied $H_{\text {Max }}$ from 10 times smaller to 1000 times larger than the 
exocytosis rate $\alpha_{E x_{0}}$ and the characteristic homotypic fusion range $\lambda_{z}$ between $\approx 3$ and $\approx$ $300 \mathrm{~nm}$. Fig. 4.5e-f shows the key characteristics of the exocytosis quantal size distributions for the $1 \mathrm{D}$ and $2 \mathrm{D}$ simulations.

In the $1 \mathrm{D}$ model, the exocytic mean quantal content $M$ increased with homotypic fusion rate $H_{M a x} / \alpha_{E x o}$ and with length constant $\lambda z$. The $C V$ of the distribution, however, exhibited a more complex dependence, but for $M>2$, the $C V$ decreased with increasing $H_{M a x} / \alpha_{E x o}$ and decreasing $\lambda_{z}$. In this region, the skewness showed a similar behavior. Where the ratio of uniquantal to most frequent events equaled 1, the mode of the distribution was at 1 quantum, i.e. most events were uniquantal. We found that this ratio decreased with increasing $H_{M a x}$, demonstrating a decrease in the fraction of uniquantal events. The consistency region (white stripes, where the model is consistent with experimental observations) extends from $H_{\text {Max }} / \alpha_{E x o}>7$. It covers mean quantal contents $M$ from around 1.5 to 7 , spanning $M$ estimates for all synapses considered (examples in Fig. 4.5c).

The behavior of the $\mathrm{Ca}^{2+}$ dependent homotypic fusion model radically changed when vesicles could diffuse freely on the entire $2 \mathrm{D}$ ribbon surface (Fig. $4.5 d, f)$. In fact, the $\mathrm{Ca}^{2+}$ dependence of homotypic fusion rate did not any longer prevent homotypic fusion from being a self-amplifying process but instead, the model either generated vesicles of unrealistically large size or vesicle quantal content distributions with a mode at 1. First, although the mean quantal content $M$ depended on $H_{M a x} / \alpha_{E x o}$ and $\lambda_{z}$ in a similar way as in 1D, it exhibited higher values for the same parameter values. Second, the region of runaway homotypic fusion (white space) was more prominent in the $2 \mathrm{D}$ case. Third, the $C V$ of the quantal content distribution was larger and mainly above 0.4. Finally the large skewness and ratio of uniquantal to most frequent events of 1 indicate that the mode of the distribution was almost always at 1 quantal. As a result, the consistency region disappeared completely. 
$\mathrm{Ca}^{2+}$ dependent homotypic fusion model of multiquantal release (MQR)

a

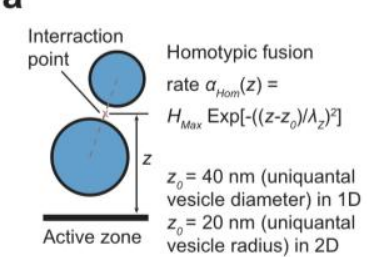

b

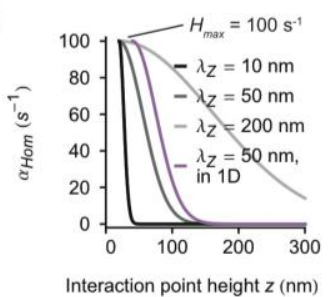

c

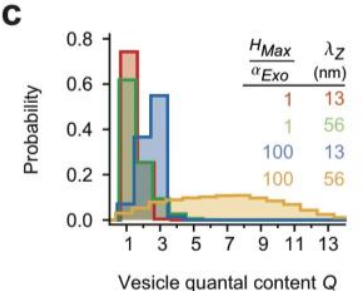

d

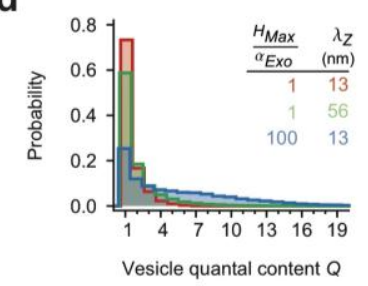

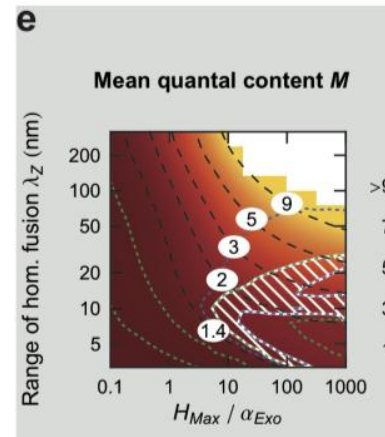

f

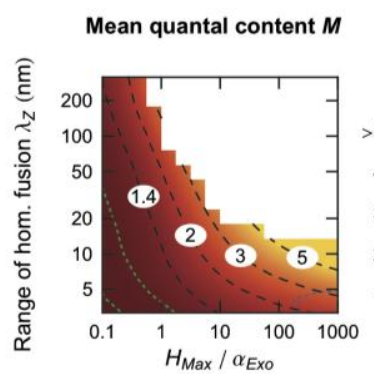

1 dimensional top-down vesicle track
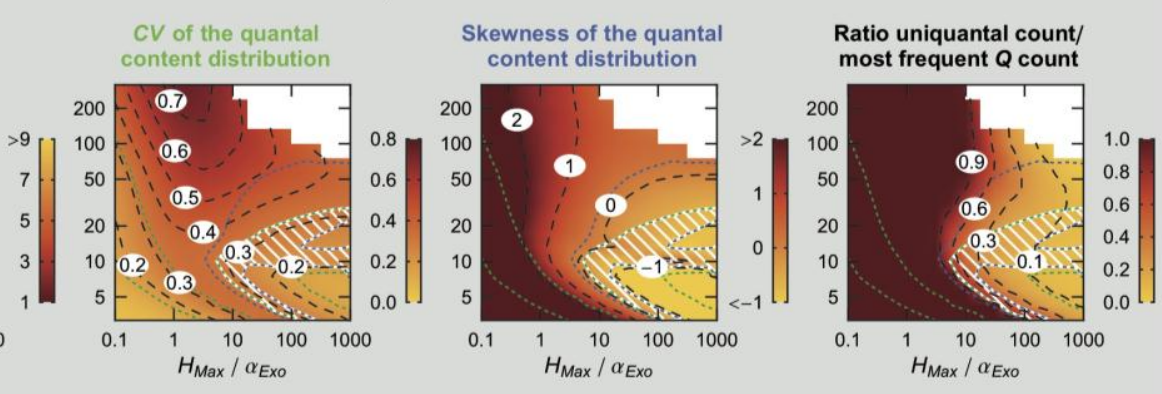

Vesicles on the 2 dimensional ribbon surface
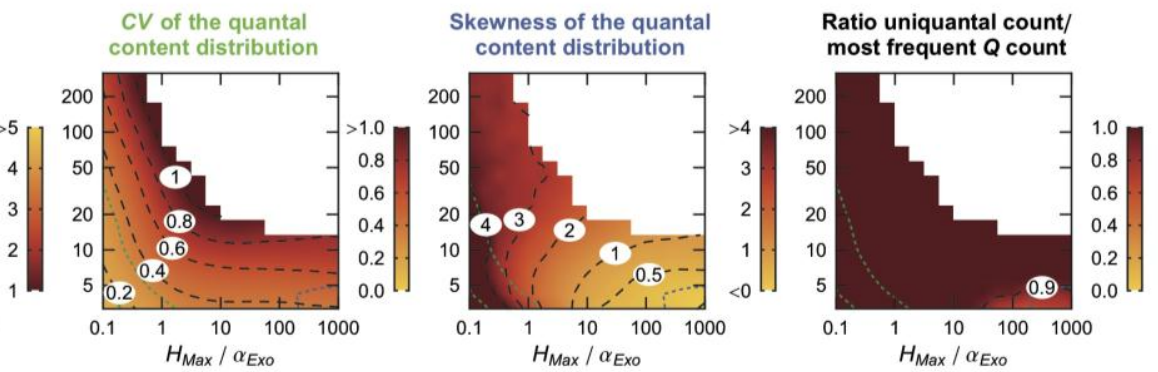

Figure 4.5: $\mathrm{Ca}^{2+}$ dependent homotypic fusion model for MQR

(a) To model the $\mathrm{Ca}^{2+}$ dependence of homotypic fusion, homotypic fusion rates decreased with the distance of the vesicle interaction point $z$ from the active zone. $H_{\text {Max }}$ is the maximum homotypic fusion rate, $\lambda_{Z}$ is the decay length of where homotypic fusion happens from the active zone.

(b) Examples of homotypic fusion rate decreasing with the distance from the membrane, for different values of $\lambda_{Z}$ and $H_{\text {Max }}=100 \mathrm{~s}^{-1}$.

(c) exocytic quantal content histogram for different values of $H_{\text {Max }} / \alpha_{\text {exo }}$ and $\lambda_{z}$ in the 1D model (Fig. 4.4a). These histograms quantitatively reproduced those observed experimentally.

(d) Same as (c) in the 2D model (Fig. 4.4b). These histograms did not reproduce those observed experimentally, either due to the high skewness of the distribution or due the emergence of extremely large vesicles.

(e),(f) Critical characteristics of the exocytic quantal content distributions in 1D and 2D models as a function of $H_{\text {Max }} / \alpha_{E x O}$ and $\lambda_{Z}$. Note that both parameters are in logarithmic scale. The consistency region (white stripes) corresponds to where the model reproduces experimental observation (Table 4.1), the criteria being in terms of $C V$ (green dashed lines) and skewness (blue dashed lines) of the quantal content distribution. The runaway fusion area (plain white) indicate simulations that were discarded due to the formation of vesicle with quantal content $Q$ $>40$ (diameter: $252 \mathrm{~nm}$ ).

(e) 1D model. A consistency region is present for $H_{\text {Max }} / \alpha_{\text {exo }}>7$. Decreasing the extend of the $\mathrm{Ca}^{2+}$ signal, modeled by $\lambda_{Z}$, produces a switch from multiquantal release to uniquantal release. The areas excluded from the consistency region at the right of the parameter space arise from strongly negatively skewed $\left(\gamma_{1} \approx-1\right)$ quantal content histogram.

(f) $2 \mathrm{D}$ model. No consistency region is present as the mode of the exocytic quantal content distribution is almost everywhere 1 . A very large area of runaway fusion exists.

The vesicle packing densities $\varphi_{1 D}$ and $\varphi_{2 D}$ were 0.7 and 0.4 , respectively. 
This qualitative difference between the 1D and 2D homotypic fusion models resulted from two main mechanisms. Firstly, larger vesicles in 2D had more partners and therefore an increased probability to grow further by homotypic fusion, which was not the case in 1D. Secondly, distinct from the 1D geometry, as a vesicle grew near the active zone, its homotypic fusion rate to a lateral neighbor vesicle does not decrease as much as to its vertical neighbor. Therefore, in 2D once homotypic fusion has commenced, the resulting compound tended to continue fusing and growing, inevitably creating positively skewed quantal content histograms. For both $1 \mathrm{D}$ and 2D scenarios, we found that the exact shape of the consistency region depended on the vesicle packing density $\varphi$. Increasing $\varphi$ shifted the consistency region slightly towards smaller homotypic fusion rates (Supplementary Fig. 4.5-4.6).

Although only the 1D homotypic fusion model could reproduce experimental observations, both $1 \mathrm{D}$ and 2D models predicted an important qualitative feature of $\mathrm{MQR}$ in frog $\mathrm{HC}$ and RBC: the shift from MQR to uniquantal release when decreasing the stimulation strength or adding stronger buffer in the presynaptic solution (Singer et al., 2004; Li et al., 2009). In the model this condition was realized by reducing $H_{M a x} / \alpha_{E x o}$ and $\lambda_{z}$, because of the spatially less extended $\mathrm{Ca}^{2+}$ signal.

Our results thus indicate that unconstrained homotypic fusion cannot reproduce the experimentally observed featured of MQR in ribbon synapses. If the molecular architecture of the ribbon constrains vesicles to diffuse anisotropically, such that they move mostly vertically to the AZ, and perform mostly vertical fusions, a solely $\mathrm{Ca}^{2+}$ and geometrically controlled homotypic fusion process could explain the observed features of MQR. But in view of the complex and rich proteome of synaptic vesicles (Takamori et al., 2006), it appears likely that the homotypic fusion process is regulated even further by molecular means. 


\subsubsection{Homotypic fusion dependent on $\mathrm{Ca}^{2+}$ and vesicle size}

A molecular regulation mechanism could decrease homotypic fusion rates as quantal content $\mathcal{Q}$ increases: e.g., by consumption of proteins need for fusion. Therefore, in the final homotypic fusion model, we made homotypic fusion rates dependent both on the distance $z$ from the synaptic membrane as well as on the quantal contents $Q$ of the parent vesicles (Fig. 4.6a,b):

$$
\alpha_{\text {Hom }}\left(z, Q_{1}, Q_{2}\right)=H_{\text {Max }} e^{-\left(\frac{z-z_{0}}{\lambda_{z}}\right)^{2}} \theta\left(z-z_{0}\right) e^{-\left(\frac{Q_{1}+Q_{2}-2}{\lambda_{Q}}\right)^{2}} \theta\left(Q_{1}+Q_{2}-2\right)
$$

$Q_{1}$ and $Q_{2}$ are the quantal contents of the two interacting vesicles. A uniquantal vesicle has $Q=1$, one formed by fusion of $n$ vesicles has $Q=n$. $\lambda_{Q}$ is the vesicle quantal content range of homotypic fusion - it determines how fast homotypic rates decreases with quantal content $Q$. This may be a parameter fixed by the vesicles intrinsic properties and fusogenic protein complements (Takamori et al., 2006). The functional form was chosen for simplicity and to implement a rapid rate decrease with vesicle quantal content. We examined a range of $\lambda_{Q}$ from 0 to 5.4 .

We found an extended consistency region (Fig. 4.6c,e, white stripes). No runaway fusion region was present in this parameter range, demonstrating that the selfamplifying process of homotypic fusion was effectively prevented. The mean exocytic quantal content $M$ increased with $H_{\operatorname{Max}} / \alpha_{E \times 0}$ and $\lambda_{Q}$. To obtain $M>1, H_{M a x}$ needed to be larger than $\alpha_{E x x}$, because homotypic fusion was constrained to happen close to the membrane (here $\lambda_{Z}=100 \mathrm{~nm}$ ). The dependencies of the other parameters largely resembled the behavior in the previous $\mathrm{Ca}^{2+}$-dependent 1D model (Fig. 4.6e). In conclusion, a decrease of homotypic fusion rates with vesicle quantal content seems sufficient to quantitatively explain several features of MQR in ribbon synapses.

During most physiological conditions, the base of ribbon should contain significantly more multiquantal vesicles than uniquantal (Fig. 4.6d). This is strongly in contrast to most EM studies, which only rarely observed larger compounds (Matthews and Sterling, 2008). To account for this rare visibility of large vesicles, vesicles with greater quantal content should exhibit a higher rate of exocytosis. This, together with requirement of a lower rate of homotypic fusion seems difficult to implement biophysically, provided that both fusion reaction probably use similar fusogenic proteins. 
Vesicle quantal content and $\mathrm{Ca}^{2+}$ dependent homotypic fusion model of multiquantal release (MQR), vesicle moving on the 2 dimensional ribbon surface

a

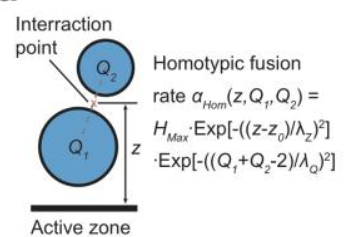

e

Mean quantal content $M$

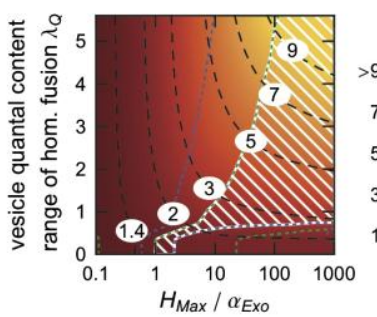

b
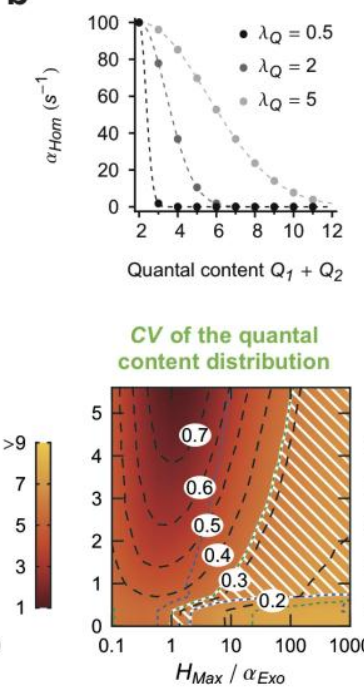

$C V$ of the quantal
C

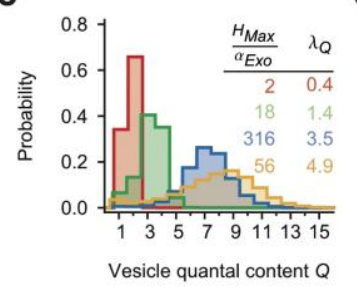
content distribution

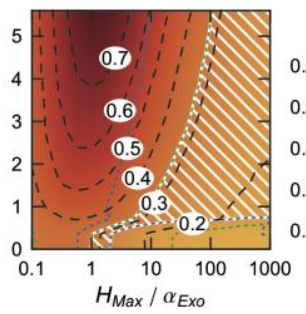

Skewness of the quantal content distribution d
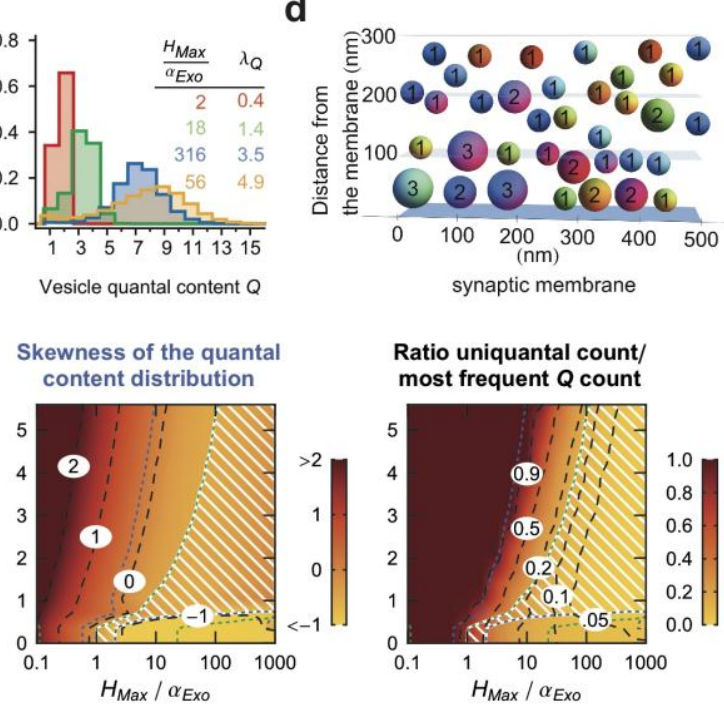

Ratio uniquantal count/ most frequent $Q$ count

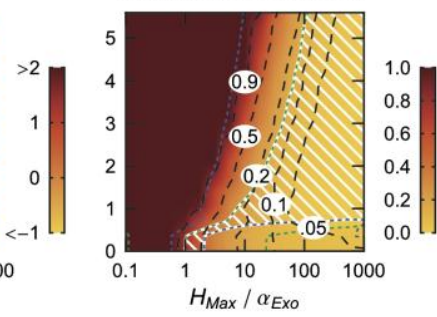

Figure 4.6: Vesicle quantal content and $\mathrm{Ca}^{2+}$ dependent homotypic fusion model for MQR

(a) To model the vesicle quantal size and $\mathrm{Ca}^{2+}$ dependence of homotypic fusion, homotypic fusion rates decreased with the vesicles quantal content as well as on the distance of the interaction point from the active zone. $H_{\text {Max }}$ is the maximum homotypic fusion rate, $\lambda_{Q}$ indicates how rapidly homotypic fusion rates decrease with the vesicle quantal content and $\lambda_{Z}$ is the decay length of where homotypic fusion happens from the active zone

(b) Examples of homotypic fusion rate decreasing with the sum of the quantal contents of the parent vesicles, for different values of $\lambda_{Q}$ and $H_{\text {Max }}=100 \mathrm{~s}^{-1}$.

(c) Exocytic quantal content histogram for different values of $H_{\text {Max }} / \alpha_{\text {exo }}$ and $\lambda_{Q}$ in the 2D model (Fig. 4.4b) with $\lambda_{Z}=100 \mathrm{~nm}$. These histograms quantitatively reproduce those observed experimentally for each system.

(d) Snapshot of a homotypic fusion simulation with $H_{\text {Max }} / \alpha_{\text {exo }}=10, \lambda_{Q}=1, \lambda_{Z}=100 \mathrm{~nm}$. The exocytic quantal content histogram resulting from such a simulation is consistent with the histogram from the frog $\mathrm{HC}$.

(e) Critical characteristics of the exocytic quantal content distributions for the $2 \mathrm{D}$ model as a function of $H_{\text {Max }} / \alpha_{E x O}$ and $\lambda_{Q}$. Only the former is in logarithmic scale. The consistency region (white stripes) corresponds to where the model reproduces experimental observation (Table 4.1), the criteria being in terms of $C V$ (green dashed lines) and skewness (blue dashed lines) of the quantal content distribution. The runaway fusion area (plain white) indicate simulations that were discarded due to the formation of vesicle with quantal content $Q>40$ (diameter: 252 $\mathrm{nm})$. An extended consistency region is present starting at $H_{\operatorname{Max}} / \alpha_{\text {exo }}>1$. The fraction of uniquantal events was never negligible near the left border of the consistency region and for mean quantal content $M>3$, where we observed a high ratio (>10\%) of uniquantal to most frequent events. This results from the fact that mainly large non-fusogenic vesicles were present near the active zone (AZ) and uniquantal vesicles could squeeze through and exocytosed without prior homotypic fusion. A larger $\lambda_{Z}$ would enable vesicle to fuse further from the active zone and less uniquantal vesicle would reach the $A Z$.

$\lambda_{Z}=100 \mathrm{~nm}$ for all the simulations. 


\subsubsection{Uniquantal and subquantal release interpretation}

The difficulty of both coordinated fusion and homotypic fusion scenarios to account for MQR led us to explore an alternative mechanism by modeling. We considered the plausibility of a uniquantal interpretation, where large EPSCs would result from the complete fusion of only one vesicle. Small EPSCs would appear as a consequence of partial (subquantal) release: a short opening of the vesicle fusion pore during which only a fraction of the vesicle neurotransmitter content would escape into the synaptic cleft (Singer et al., 2004; He et al., 2006). This hypothesis has not been considered for the IHC due to the presence of multiphasic EPSCs and due to the unusually large EPSC size (mean around 300-400 pA) (Grant et al., 2010). It was neither considered in the frog HC due to large EPSC amplitude (100-200 pA) as well as due to the possibility to reduce the mean EPSC size by holding the $\mathrm{HC}$ at very hyperpolarized membrane potentials. We examined whether a single vesicle could generate such large EPSCs and if short pore flickers could generate EPSCs of smaller sizes.

To understand if a single synaptic vesicle could trigger large EPSCs, we simulated the instantaneous release of the neurotransmitter content of a vesicle loaded with 4000 glutamate molecules in the center of the postsynaptic density (Trommershäuser et al., 1999; Pawlu et al., 2004; He et al., 2006; Savtchenko and Rusakov, 2007). The diffusion coefficient of glutamate was assumed $0.33 \mu \mathrm{m}^{2} / \mathrm{ms}$ (Nielsen et al., 2004) and the width of the synaptic cleft was set to $10 \mathrm{~nm}$ (Pawlu et al., 2004). We used the analytical solution of (Savtchenko and Rusakov, 2007) to calculate the time course of the mean glutamate concentration in the synaptic cleft as well as the mean open probability $P_{\text {open }}$ of the AMPA receptors for different sizes of postsynaptic clusters (Fig. 4.7a). We found that the maximum mean $P_{\text {open }}$ was around 0.55 (consistent with previous simulations (Trommershäuser et al., 1999)) and decreased only slightly with cluster size. The decrease of $P_{\text {open }}$ results from the smaller mean glutamate concentration at the postsynaptic cluster when the cluster is larger. While the concentration is the highest at the point of release, the decrease towards the periphery is minute, meaning that the glutamate concentration is high enough for post-synaptic density of $500 \mathrm{~nm}$ radius. 
a

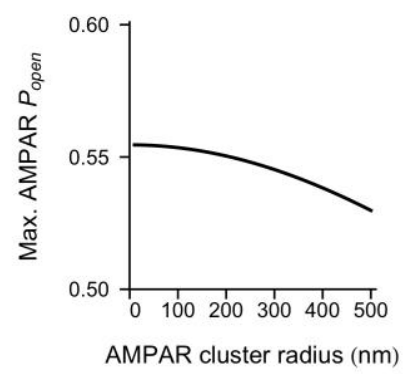

b

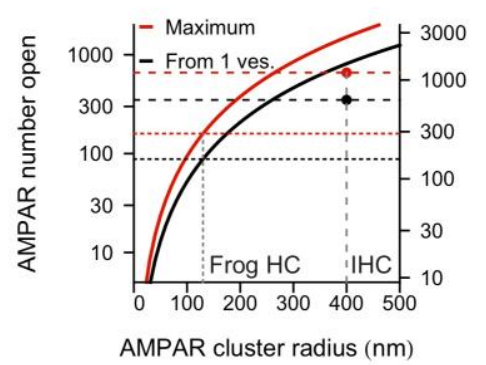

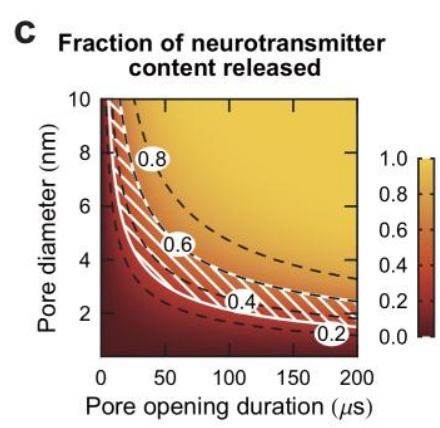

Figure 4.7: Vesicle pore flickering could explain different EPSC sizes and large postsynaptic cluster could explain large miniature EPSC.

(a) Maximum mean open probability of the AMPA receptor during the time course of an EPSC in result to the exocytosis of a single vesicle (4000 glutamate molecules) decreases very slightly with the AMPA cluster size.

(b) Number of open AMPAR deduced from the open probability (left axis), assuming a 3000 receptors per $\mu \mathrm{m}^{2}$. Right axis: EPSC size deduced from the number of open AMPAR, assuming $20 \mathrm{pS}$ per AMPAR and $90 \mathrm{mV}$ holding potential. The AMPAR cluster in the IHC has a donut shape. The dots are the predicted response assuming a total receptor cluster radius of $400 \mathrm{~nm}$ and a receptor-free hole of $300 \mathrm{~nm}$. The red line corresponds to the total number of AMPAR in the cluster and the maximum EPSC size.

(c) Fraction of neurotransmitter that would escape from a vesicle for different pore opening durations and diameters. The striped area corresponds to 30 to $60 \%$ of fraction escaped.

We then used the calculated mean open probability to derive the maximum number of open AMPAR during an EPSC as well as the EPSC amplitude. We assuming a AMPAR channel conductance of 20 pS (Sahara and Takahashi, 2001) and 3000 receptors per $\mu_{\mathrm{m}}^{2}$ (Saito, 1990; Pawlu et al., 2004) (Fig. 4.7b). We also plotted the number of receptor available and the maximum possible EPSC size. The dots in Fig. $4.7 \mathbf{b}$ represent the response in the case of the IHC, which postsynaptic cluster is donut-shaped (Meyer et al., 2009). We chose a cluster radius of $120 \mathrm{~nm}$ for the frog HC (Simmons et al., 1995; Keen and Hudspeth, 2006; Li et al., 2009). We found that a single vesicle could elicit EPSC sizes of similar amplitudes to the "multiquantal" EPSC, and that the maximum EPSC size is consistent with the largest EPSCs recorded in both systems. This suggests that one vesicle could generate large EPSCs.

Next, we investigated whether small "uniquantal" EPSCs could result from subquantal release due to a short opening of the vesicle fusion pore. We simulated the diffusion of glutamate out of a vesicle and calculated the fraction of the neurotransmitter content escaping in the synaptic cleft for different pore diameters and pore opening durations (Fig. 4.7c). We considered duration smaller than $200 \mu$ s to be consistent with the observed EPSC rise times. The consistency region corresponds to the parameters needed to reproduce the small EPSC found in the frog $\mathrm{HC}$ and $\mathrm{RBC}$, where they 
represent around 30 to $60 \%$ of the large EPSC. We found that half of a vesicle's neurotransmitter content could escape from a vesicle through a pore of $4 \mathrm{~nm}$ diameter in only $50 \mu \mathrm{s}$. If vesicle fusion pores opening durations and sizes are rather invariable, this could account for the small EPSC distributions seen in frog HC and RBC. Additionally, multiphasic EPSC observed in the IHC could be due to small and short pore flickers. Prolonged pore opening with small radius would lead to EPSCs shapes that are not readily explained by a superposition of miniature EPSCs (Pawlu et al., 2004). In summary, the uniquantal/subquantal hypothesis is a plausible alternative to account for the electrophysiological data found in ribbon synapses. 


\subsection{Discussion}

In this study we explored the principles and constrains governing two alternative candidate mechanisms of $\mathrm{MQR}$ at ribbons synapses: $\mathrm{Ca}^{2+}$ coordinated release and homotypic fusion. Using a modeling approach we identified critical parameters constraining these mechanisms. We found that biophysical models of both mechanisms could barely reproduce the experimentally observed features of transmitter release over a broad range of parameters tested. This was particularly the case for the synapses of cochlear IHCs. This led us to search for alternative interpretations of electrophysiological data. We proposed that the uni- and subquantal release as a candidate mechanism.

The quality of $\mathrm{Ca}^{2+}$ coordination of $\mathrm{MQR}$ is limited by the rate of the final release step. The required $\mathrm{Ca}^{2+}$ concentrations and $\mathrm{Ca}^{2+}$ channel open times that could generate experimentally indicated synchronization of MQR seem unlikely to be met in physiology, even under artificially favorable conditions considered in the model: rectangular $\mathrm{Ca}^{2+}$ pulses, simultaneous exposure of the vesicles to exactly the same concentration and enhancing the $\mathrm{Ca}^{2+}$ sensor's final fusion rate beyond the experimentally observed values. Moreover, the synchrony we considered in this study is an underestimation of the actual synchrony needed to account for monophasic release evens. In fact, the rise-times of measured EPSC are probably limited by dendritic filtering. The theoretical minimum predicted by simulations is in the order of $0.1 \mathrm{~ms}$ (Bartol et al., 1991) and corresponds to the rise-time measured for monophasic EPSCs at the IHC synapse, where the recording is made from the synaptic bouton. The EPSC rise times at the frog $\mathrm{HC}(\approx 0.2 \mathrm{~ms})$ and at the $\mathrm{RBC}(\approx 0.3 \mathrm{~ms})$ are longer, which could result from the fact that the electrode is further away from the postsynaptic receptor cluster. This, together with the findings that low temperatures did not cause jitter or slowing of the postsynaptic currents ( $\mathrm{Li}$ et al., 2009) and the same EPSC size and kinetics were observed in conditions where the ready releasable pool is not fully replenished, led us to argues against coordination of release by a single $\mathrm{Ca}^{2+}$ channel current as a likely candidate for MQR. This prediction could further be tested by combining pre- and postsynaptic recording with homogeneous elevation of $\left[\mathrm{Ca}^{2+}\right]$ in the presynapse (e.g. by $\mathrm{Ca}^{2+}$ uncaging). If highly synchronized MQR was still observed under a steady background $\left[\mathrm{Ca}^{2+}\right]$, this would rule out $\mathrm{Ca}^{2+}$ pulse coordination as a mechanism of MQR. $\mathrm{Ca}^{2+}$ coordinated release could however still explain MQR when multiple $\mathrm{Ca}^{2+}$ channel open simultaneously upon a rapid receptor potential change. Moreover, it could account for multiphasic EPSCs observed in the IHC. 
The homotypic fusion model could reproduce electrophysiologically observed features of MQR when homotypic fusion rates decreased with vesicle quantal content. This regulatory effect of quantal size might result from the dilation or consumption of available fusogenic proteins on the vesicle surface. Also homotypic fusion rates would be $\mathrm{Ca}^{2+}$ dependent in the case of the $\mathrm{RBC}$ and the frog $\mathrm{HC}$, but $\mathrm{Ca}^{2+}$ independent in the case of the IHC. Homotypic fusion rates should be slightly higher than exocytosis rates and variations in the $\mathrm{Ca}^{2+}$ profile (represented by $\lambda z$ and $H_{M a x}$ in the model) can cause a transition from uniquantal to multiquantal release. This is consistent with the observations in the frog $\mathrm{HC}$ and $\mathrm{RBC}$, where uniquantal release is achieved either by decreasing extracellular $\mathrm{Ca}^{2+}$, hyperpolarizing the membrane potential or increasing the intracellular $\mathrm{Ca}^{2+}$ buffering.

On the other hand, to account for the scarcity of compounds vesicle in electron microscopy images, exocytosis rates in the homotypic fusion model should increase with vesicle quantal content. If the same proteins govern homotypic and plasma membrane fusion rates, it seems difficult if not impossible to implement an increase of exocytosis rates and a decrease of the homotypic fusion rates with vesicle size. For the RBC, where the maximum quantal content seems to be 2 at most (Singer et al., 2004), the small difference in vesicle diameter (40 nm for a uniquantal versus $57 \mathrm{~nm}$ for two quanta) might have escaped attention in the EM data. Finally, the homotypic fusion model would not explain the occurrence of multiphasic EPSCs in IHC. This set of evidence led us to consider alternative mechanisms to explain electrophysiological findings at ribbon synapses.

Using biophysical modeling we conclude that the neurotransmitter content of a single vesicle would be sufficient to account for the large EPSCs found in IHC and frog HC. Also, we found that a short opening of the fusion pore could explain the small EPSCs observed in frog $\mathrm{HC}$ and RBC. Finally, multiple pore flickers could be underlying the multiphasic EPSCs in the IHC. In IHCs, such pore flickering seems to become unlikely with the maturation of the synapse (Grant et al., 2010). In the frog HC, partial fusion would operate only at unphysiologically hyperpolarized potentials. In summary we regard that the uniquantal hypothesis should be considered as a plausible alternative.

Could the special exocytosis machinery present at IHC produce such unusual pore flickering? (Nouvian et al., 2011) Or could the ribbon play a role in the modulation of fusion pore dynamics? Whereas these questions cannot be answered by the present work, it will stimulate further investigation, for example using on-cell capacitance measurements, of the mechanisms of exocytosis at ribbon synapses. 


\subsection{Methods}

\subsection{1 $\mathrm{Ca}^{2+}$-triggered synchronized exocytosis model}

Each of $\mathrm{N}_{A}$ vesicles' $\mathrm{Ca}^{2+}$ sensor is assumed to be exposed to a rectangular $\mathrm{Ca}^{2+}$ pulse of concentration $\left[\mathrm{Ca}^{2+}\right]_{\text {pulse }}$ and duration $D_{\text {pulse }}$, mimicking the opening of an adjacent $\mathrm{Ca}^{2+}$ channel. The concentration time course thus is:

$$
\left[\mathrm{Ca}^{2+}\right](t)=\left\{\begin{array}{cc}
0 & t<0 \mathrm{~ms} \\
{\left[\mathrm{Ca}^{2+}\right]_{\text {pulse }}} & 0 \mathrm{~ms} \leq t<D_{\text {pulse }} \\
0 & t \geq D_{\text {pulse }}
\end{array}\right.
$$

We calculated the mean number of vesicles released $N_{R}$ during such pulses and the release asynchrony $T_{\text {asynch }}$, defined as the mean 2 vesicles exocytosis time lag. This measure is a lower bound to the mean time lag between the release of first and the last vesicle during a release event. Based on established $\mathrm{Ca}^{2+}$-dependent release schemes ((Beutner et al., 2001) for the hair cells, (Schneggenburger and Neher, 2000) for the $\mathrm{RBC}$ ), we numerically calculated the mean field evolution of the sensors states by solving for the occupancy $S_{i}(t)$ of states, where each state is related to the number of $\mathrm{Ca}^{2+}$ bound to the sensor and $S_{7}$ is the released state. All simulations started from the initial condition $S_{i}(0 \mathrm{~ms})=\delta_{i 1}$ for $i=1$ to 7 , where $\delta_{i j}$ is the Kronecker delta.

We solved the system of differential equations for $S_{i}(t)$ with NDSolve in Mathematica (Wolfram Research) 7.0.1.0 on the two segments $0 \leq t \leq D_{\text {pulse }}$ and $D \leq t \leq$ $D_{\text {pulse }}+10 \mathrm{~ms}$ separately. The discontinuity of $\left[\mathrm{Ca}^{2+}\right](t)$ at $t=D_{\text {pulse }}$ was thus treated by taking the final states' of the first segment as the initial condition for the second segment. NDSolve by default uses an LSODA approach, switching between a non-stiff Adams method and a stiff Gear backward differentiation formula method. Error tolerance in NDSolve was set to $10^{-20}$.

The vesicular release probability $P_{R}$ was taken as $P_{R}=S_{7}\left(D_{\text {pulse }}+10 \mathrm{~ms}\right)$. We derived the mean vesicle number released $N_{R}$ using binomial statistics, neglecting norelease events:

$$
N_{R}\left(N_{A}, P_{R}\right)=\sum_{k=1}^{N_{A}} k f\left(k ; N_{A}, P_{R}\right) / \sum_{k=1}^{N_{A}} f\left(k ; N_{A}, P_{R}\right)=\frac{N_{A} P_{R}}{1-\left(1-P_{R}\right)^{N_{A}}}
$$

where $f(k ; n, p)=\frac{n !}{k !(n-k) !} p^{k}(1-p)^{k}$ is the probability mass function of the binomial distribution $B(n, p)$. 
The release probability density function $f_{R}(t)$, which is also the vesicular release rate, was obtained by taking the temporal derivative of the occupancy of the last sensor state $S_{7}(t)$ (i.e., the released state): $f_{R}(t)=\frac{d}{d t} S_{7}(t)$. The probability density $P_{L}(D)$ that 2 vesicles fuse with an absolute time lag $l>0$ ms can be determined from:

$$
P_{L}(l) \propto \int_{-\infty}^{+\infty} f_{R}(t) \cdot f_{R}(t+l) d t+\int_{-\infty}^{+\infty} f_{R}(t) \cdot f_{R}(t-l) d t=2 \int_{-\infty}^{+\infty} f_{R}(t) \cdot f_{R}(t+l) d t
$$

The release asynchrony $T_{\text {asynch }}$, defined as the mean 2 vesicle release time lag is then:

$$
T_{\text {asyncb }}=\int_{0}^{+\infty} l \cdot P_{L}(l) d l
$$

We calculated (4.5) and (4.6) numerically. We sampled $f_{R}(t)$ on the interval 0 to $D_{\text {pulse }}+10 \mathrm{~ms}$ with a step of $5 \mu$ s and performed an auto-correlation (using FFT) of this list of numbers, by padding 0 on the right (maximal overhang at the right-hand end). Due to the numerical integrations scheme, when $S_{7}(t)$ saturated, $f_{R}(t)$ could slightly oscillated around 0 . In that case, the interval of interest was limited up to the first negative value of $f_{R}(t)$. This calculation gave us the probability $P_{L}(l)$ of a certain time lag $l$, up to normalization. The mean 2 vesicles release time lag $L$ was calculated using the discrete version (Riemann sum) of (4.6).

Ion channels in the simplest case have exponentially distributed open times. We calculated the mean event size $\overline{N_{R}}$ and the release asynchrony $\overline{T_{\text {asynch }}}$ for different $\mathrm{Ca}^{2+}$ concentrations $\left[\mathrm{Ca}^{2+}\right]_{p u l s e}$ and ion channel mean open times $\tau_{\text {Open. }}$. For an ion channel with a mean open time $\tau_{\text {Open }}$, the probability density $P_{D}\left(D_{\text {pulse }}, \tau_{\text {Open }}\right)$ to open for a duration $D_{\text {pulse }}$ is:

$$
P_{D}\left(D_{\text {pulse }}, \tau_{\text {Open }}\right)=\exp \left(-D_{\text {pulse }} / \tau_{\text {Open }}\right) / \tau_{\text {Open }}
$$

In the following, $\left[\mathrm{Ca}^{2+}\right]_{\text {pulse }}$ and $D_{\text {pulse }}$ are abbreviated as $C$ and $D$, respectively. For given $C$ and $\tau_{\text {Open }}$, the mean number of vesicles released $\overline{N_{R}}$ is:

$$
\overline{N_{R}}\left(C, \tau_{\text {Open }}\right)=\frac{\int_{0}^{\infty} N_{R}(C, D) \cdot P_{D}\left(D, \tau_{\text {Open }}\right) \cdot P_{1 V e s}(C, D) \cdot d D}{\int_{0}^{\infty} P_{D}\left(D, \tau_{\text {Open }}\right) \cdot P_{1 V e s}(C, D) \cdot d D}
$$

whereas the release asynchrony $\overline{T_{a s y n c b}}$ is: 


$$
\overline{T_{\text {asynch }}}\left(C, \tau_{\text {Open }}\right)=\frac{\int_{0}^{\infty} T_{\text {ayynch }}(C, D) \cdot P_{D}\left(D, \tau_{\text {Open }}\right) \cdot P_{2 V e s}(C, D) \cdot d D}{\int_{0}^{\infty} P_{D}\left(D, \tau_{\text {Open }}\right) \cdot P_{2 V e s}(C, D) \cdot d D}
$$

where $P_{1 V e s}(C, D)$ and $P_{2 V e s}(C, D)$ are the probability that at least 1 and at least 2 vesicles get released during a $\mathrm{Ca}^{2+}$ pulse of duration $D$ and concentration $C$, respectively:

$$
\begin{gathered}
P_{1 V_{e s}}(C, D)=1-\left(1-P_{R}(C, D)\right)^{N_{A}} \\
P_{2 V_{e s}}(C, D)=1-\left(1-P_{R}(C, D)\right)^{N_{A}}-N_{A} \cdot P_{R}(C, D) \cdot\left(1-P_{R}(C, D)\right)^{N_{A}-1}
\end{gathered}
$$

where $N_{A}$ is the number of vesicles sensing the pulse.

To compute (4.8) and (4.9) for given $C$ and $\tau_{\text {Open }}$ we first calculated the functions $P_{R}(C, D), P_{1 V e s}(C, D), P_{2 V e s}(C, D), N_{R}(C, D)$ and $T_{a s y n c b}(C, D)$ for pulse durations $D$ from 0.01 to $10 \mathrm{~ms}$ with a maximum step size of $0.1 \mathrm{~ms}$. Because the largest changes in $P_{\mathrm{R}}(C, D)$ and $T_{\text {asyncb }}(C, D)$ occurred for small pulse durations $D$, from 0.01 to $0.31 \mathrm{~ms}$ we used smaller logarithmic steps in $D: D=10^{d x}$ for $d x$ from -2 to -0.5 in step of 0.1 . Second, we performed a third order interpolation of all the points and resampled the interpolated function with a step of $10 \mu \mathrm{s}$. For the point $D=0 \mathrm{~ms}$, we took $P_{R}(C, D=0 \mathrm{~ms})=0$ (as we found that $\left.P_{R}(C, 0.01 \mathrm{~ms})<10^{-6}\right)$ and $T_{a s y n c b}(C, D=0)=L(C, D=0.01 \mathrm{~ms})$. Finally we calculated the integrals in (6) and (7) as a Riemann sum using the resampled points. An ion channel with $\tau_{\text {Open }}=2 \mathrm{~ms}$ has $99 \%$ of its openings shorter $10 \mathrm{~ms}$. Terminating the calculations at $D=10 \mathrm{~ms}$ thus only slightly underestimated the release asynchrony $\overline{T_{a y y n c b}}$ and the mean number of released vesicles $\overline{N_{R}}$.

In Supplementary Fig. 4.1, we show $P_{D}\left(D, \tau_{O p e n}\right), P_{R}(C, D), P_{1 V e s}(C, D), P_{2 V e s}(C$, $D), P_{1 V e s}(C, D) \cdot P_{D}\left(D, \tau_{O p e n}\right)$ and $P_{1 V e s}(C, D) \cdot P_{2 V e s}\left(D, \tau_{O p e n}\right)$ for a representative choice of parameters.

\subsubsection{Vesicle diffusion and homotypic fusion model}

To examine the homotypic fusion scenario of MQR, we simulated the behavior of vesicles on the synaptic ribbon surface at the active zone (AZ). This incorporated their Brownian motion in a defined one-dimensional (1D) or two-dimensional (2D) geometry, their docking to the membrane, exocytosis, homotypic fusion and replenishment of the vesicle pool. Our goal was to determine the exocytosis event size histograms generated by different models of homotypic fusion and compare them to experimental observations. For diffusion we used a Langevin equation approach. It included stochastic 
forces driving diffusion and deterministic forces, which repelled vesicles from each other and from the boundaries, as well as a short-range force modeling docking that attracted the vesicles to the AZ. Two types of reactions could occur: exocytosis and homotypic fusion of vesicles. In addition refilling of the vesicles pool maintained an essentially constant vesicle packing density.

At each simulations time step, the order of processes was the following: replenishment, homotypic fusion, exocytosis and diffusion. At the end of each homotypic fusion and diffusion event, vesicles extending below the plasma membrane were translated directly above it.

The methods are essentially the same as in chapter 3, but with the addition of homotypic fusion. Therefore only the differences will be stated.

\section{Simulation volume, and vesicle pool replenishment}

Simulations volumes represented either a $1 \mathrm{D}$ column on the ribbon or the whole 2D ribbon surface (Fig. 4.4a,b). In 1D, vesicle centers moved on a line with a height $H=$ $400 \mathrm{~nm}$. In 2D, vesicle centers moved on the surface of as cylinder with a perimeter $P=$ $500 \mathrm{~nm}$ and height $H=300 \mathrm{~nm}$. The active zone always refers to the bottom of the simulation volume. Simulations were initialized with 0 vesicles in the simulation volume

Vesicles were added at a maximum rate of $2 \mathrm{kHz}$ and at least 3 vesicles were maintained in the simulation volume.

\section{The diffusion coefficient for larger vesicles}

The Einstein-Stokes equation gives us the diffusion coefficient for a spherical object of radium $R$ as a function of temperature, viscosity $\eta$ of a medium:

$$
D_{i}=\frac{k_{B} T}{6 \pi R_{i} \eta}
$$

We used (4.12) to account for the dependence of the diffusion coefficient on the vesicle radius.

\section{Boundary repulsion and attraction forces}

We modeled boundary forces so that vesicles stay inside the defined simulation volume and added a short range attracting force to the plasma membrane to mimic docking. Let 1 be the coordinate perpendicular to the synaptic membrane and $\hat{e}^{1}$ its unitary vector oriented towards the cytoplasm. The boundary force acting on a vesicle $i$ is defined as (Fig. 4.4c): 


$$
\vec{F}\left(\vec{x}_{i}, R_{i}\right)=F_{M} S\left(\vec{x}_{i}^{1}-R_{i}\right) \hat{e}^{1}+F_{A} A\left(\vec{x}_{i}^{1}-R_{i}\right) \hat{e}^{1}-F_{B} S\left(H-\vec{x}_{i}^{1}-R_{i}\right) \hat{e}^{1}
$$

with $H$ being the simulation volume height, $F_{M}=50 \mathrm{pN}, F_{B}=10 \mathrm{pN}, F_{A}=1 \mathrm{pN}$ and the attraction force:

$$
A(d)=\left\{\begin{array}{cc}
-\left(\frac{d}{\Delta x_{1}}\right)^{2} \operatorname{Exp}\left(\frac{d}{\Delta x_{2}}\right) & 0 \mathrm{~nm} \leq d \leq 20 \mathrm{~nm} \\
0 & \text { otherwise }
\end{array}\right.
$$

with $\Delta x_{1}=1 \mathrm{~nm}$ and $\Delta x_{2}=3 \mathrm{~nm} . S(d)$ is a sigmoid function:

$$
S(d)=\left\{\begin{array}{cc}
\frac{1}{1+\operatorname{Exp}\left(\frac{d}{\Delta x}\right)} & d \leq 7 \mathrm{~nm} \\
0 & \text { otherwise }
\end{array}\right.
$$

with $\Delta x=1 \mathrm{~nm}$.

The AZ membrane repulsion force is thus assumed stronger $(50 \mathrm{pN})$ than the vesicle force $(20 \mathrm{pN})$. The maximal attraction force is around 10 times weaker than the maximal force of repulsion. The forces cancel each other when the vesicle is at a distance of around $3 \mathrm{~nm}$ from the membrane. The time a vesicle stays docked in the case no other vesicle is in the simulation volume was $15 \pm 15 \mathrm{~s}$ (mean \pm s.d.).

\section{Homotypic fusion}

The homotypic fusion reaction:

$$
\text { Vesicle }_{i}+\text { Vesicle }_{j} \stackrel{\alpha_{\text {Hom }}}{\longrightarrow} \text { Vesicle }_{k}
$$

could only happen if the membranes of the 2 vesicles $i$ and $j$ are closer than $15 \mathrm{~nm}$. This is motivated by the length of the synaptobrevin molecules (Takamori et al., 2006). $\alpha_{\text {Hom }}$ was fixed or depended on the positions and the sizes of the 2 vesicles.

At each simulation time steps, for every pair $(i, j)$ of vesicles for which:

$$
\left|\vec{x}_{i}-\vec{x}_{j}\right|-R_{i}-R_{j}<15 \mathrm{~nm}
$$

a random number was drawn. If it was smaller than the probability $p_{H o m}=\delta t \alpha_{\text {Hom }}$, where $\delta t$ is the simulation time step, homotypic fusion occurred. For the simulation time step used, this probability was always much smaller than 1 . When required, the height $z$ of vesicle interaction was calculated as following:

$$
z=\vec{x}_{i}+\frac{\vec{x}_{j}-\vec{x}_{i}}{\left|\vec{x}_{j}-\vec{x}_{i}\right|}\left(\mathrm{R}_{i}+\frac{\left|\vec{x}_{j}-\vec{x}_{i}\right|-R_{i}-R_{j}}{2}\right)
$$


When homotypic fusion happened, the 2 parent vesicles $i$ and $j$ disappeared and a new vesicle $k$ emerged in their center of mass:

$$
\vec{x}_{k}=\frac{\vec{x}_{i} \cdot R_{i}^{3}+\vec{x}_{j} \cdot R_{j}^{3}}{R_{i}^{3}+R_{j}^{3}}
$$

with quantal size $Q_{k}=Q_{i}+Q_{j}$ and radius $R_{k}=\sqrt{R_{i}^{2}+R_{j}^{2}}=\sqrt{Q_{k}} R_{0}$ with $R_{0}=20 \mathrm{~nm}$. Thus the total lipid bilayer of the parent vesicles was preserved and their neurotransmitter content was summed. The newly created vesicle could potentially overlap with an already existing vesicle. Due to the vesicle forces, such vesicles were rapidly pushed apart.

\section{Exocytosis}

The exocytosis reaction:

$$
\text { Vesicle }+ \text { Membrane } \stackrel{\alpha_{\text {Exo }}}{\longrightarrow} \text { Membrane }
$$

could happen when the vesicle membrane was closer than $15 \mathrm{~nm}$ from the active zone surface. When it was the case, a random number was drawn and if it was smaller than the probability $p_{E x o}=\delta t \alpha_{E x o}$, where $\delta t$ is the simulation time step, exocytosis occurred. For the simulation time step used, this probability was always much smaller than 1. After exocytosis, the vesicle was removed from the simulation volume. The exocytosis rate was fixed to $3 \mathrm{~Hz}$.

\begin{tabular}{|c|c|}
\hline Parameter & Value and remarks \\
\hline Simulation volumes & $\begin{array}{l}\text { 1D: height } H=400 \mathrm{~nm} \text {, referred as the } Z \text { direction } \\
\text { 2D: height } H=300 \mathrm{~nm} \text {, referred as the } Z \text { direction, perimeter } P= \\
500 \mathrm{~nm} \text {, referred as the } X \text { direction. }\end{array}$ \\
\hline Uniquantal vesicle radius & $20 \mathrm{~nm}$ \\
\hline Diffusion coefficient & $\begin{array}{l}50 \mathrm{~nm}^{2} / \mathrm{ms} \text { for a } 20 \mathrm{~nm} \text { radius vesicles. Decreasing with vesicle radius } \\
\text { according to the Einstein-Stokes equation (4.12). }\end{array}$ \\
\hline Time step $\delta t$ & $0.01 \mathrm{~ms}$. \\
\hline $\begin{array}{l}\text { Replenishment of the vesicle } \\
\text { pool }\end{array}$ & $\begin{array}{l}\text { Maximum rate of } 2 \mathrm{kHz} \text {, so that a minimum filling fraction } \varphi_{0} \text { of the } \\
\text { volume is maintained. The number of vesicles in the simulation } \\
\text { volume was maintained to always be at least } 3 \text {. New vesicles are } \\
\text { placed directly above the simulation volume. The } \mathrm{X} \text { position is } \\
\text { chosen randomly among } 10 \text { positions in the } 2 \mathrm{D} \text { case }\end{array}$ \\
\hline Filling fraction $\varphi$ & $\begin{array}{l}\text { 1D: } 0.3,0.7,0.95 \text {. The theoretical maximum in } 1 \mathrm{D} \text { is } 1 . \\
\text { 2D: } 0.25,0.4,0.55 \text {. The theoretical maximum with dense } 2 \mathrm{D} \text { spheres }\end{array}$ \\
\hline
\end{tabular}

\section{Parameters summary used for simulations}




\begin{tabular}{ll}
\hline & packing is around 0.9. \\
\hline Exocytosis rate $\alpha_{E \times o}$ & $3 \mathrm{~Hz}$ \\
\hline $\begin{array}{l}\text { Maximum homotypic fusion rate } \\
H_{\text {Max }}\end{array}$ & From 0.1 to $3000 \mathrm{~Hz}$ \\
\hline $\begin{array}{l}\text { Decay length } \lambda_{z} \text { of homotypic } \\
\text { fusion rate }\end{array}$ & From 3 to $300 \mathrm{~nm}$. \\
\hline $\begin{array}{l}\text { Homotypic fusion decaying } \\
\text { vesicle quantal size constant } \lambda_{Q}\end{array}$ & $\begin{array}{l}\text { Infinity in the case when homotypic fusion is only dependent on the } \\
\text { distance from the membrane. From } 1 \text { to } 40 \text { otherwise. }\end{array}$ \\
\hline Initial number of vesicles & 0 \\
\hline Simulation stop condition & When 1100 and 1200 exocytosis event occurred in $1 \mathrm{D}$ and in $2 \mathrm{D}$ \\
& respectively. Or before is a vesicle with quantal content Q > 40 is \\
& created. \\
\hline
\end{tabular}

For each point in the parameter space (Fig. 4.4-6), 25 simulation repetitions were performed. If one of the simulation experienced the creation of a vesicle with $Q>40$, this parameter point was considered as runaway fusion. Otherwise from the 1100 (1200 in $2 \mathrm{D}$ model) exocytosis events, the last 1000 were taken to construct the exocytosis histograms.

Simulations were performed in $\mathrm{C}++$ and analyzed in Mathematica (Wolfram Research). Random number generator from (Press et al., 2007).

\subsubsection{Glutamate diffusion and binding to the AMPAR receptors}

We wanted to simulate the response of the postsynapse to the release of vesicle neurotransmitter. As calculated in (Savtchenko and Rusakov, 2007), the evolution of the mean glutamate concentration $C^{*}$ in the synaptic cleft is given by:

$$
C^{*}\left(r_{a}, \delta, t\right)=\frac{N}{\pi \delta r_{a}^{2}}\left(1-\exp \left(-\frac{r_{a}{ }^{2}}{4 D t}\right)\right)
$$

where $r_{a}$ is the radius of the active zone, $\delta$ is the height of the synaptic cleft, $D$ the diffusion coefficient of glutamate, $N$ the number of glutamate molecule released and $t$ is time.

Then, using the reduced scheme of (Savtchenko and Rusakov, 2007) based on (Jonas et al., 1993) for the AMPA receptor:

$$
2 G l u+A R \underset{k_{o f f}}{\stackrel{k_{o n}}{\rightleftarrows}} G l u_{2} A R \underset{\beta}{\stackrel{\alpha}{\rightleftarrows}} O
$$

We calculated the mean open probability of the AMPAR in the cluster. Glu stands for the glutamate molecule, $A R$ an AMPA receptor and $O$ is the open state of an AMPA 
receptor. This model does not take into account the decrease in glutamate concentration resulting from glutamate binding to the receptors (Trommershäuser et al., 1999). This is a good approximation when the number of glutamate used is a small proportion of the total available. In the case of large number of AMPA receptor, this approximation gives an overestimate of the real amount of open channels.

\subsubsection{Neurotransmitter escape from the synaptic vesicle through a fusion pore}

We modeled a vesicle as a cylinder of $17.5 \mathrm{~nm}$ radius and $35 \mathrm{~nm}$ height. Its volume $\left(33700 \mathrm{~nm}^{3}\right)$ corresponds roughly to the volume of a $20 \mathrm{~nm}$ radius vesicle $\left(33500 \mathrm{~nm}^{3}\right)$. In the beginning of the simulations the concentration inside the vesicle what set to 1 . Then a pore of a given diameter was opened at the base of the cylinder. The length of the pore was $8 \mathrm{~nm}$ (Bartol et al., 1991) and anything that reached the end of the pore was set as escaped into the synaptic cleft. This was achieved mathematically by setting an absorbing boundary condition at the end of the pore. We then monitored the concentration inside the vesicle during the time course. Anything that was inside the pore and the vesicle was considered to stay in the vesicle once the pore closed. That way we deduced the fraction of neurotransmitter that escaped from the vesicle for different durations and through different pore diameters.

The calculations were done using CalC (Matveev et al., 2002), which use mean field evolution of the concentration in cylindrical coordinates. The grid was $175 \times 175$ points in the $\mathrm{R}$ direction and in the $\mathrm{Z}$ direction, respectively. The diffusion of the glutamate was assumed as before $0.33 \mu \mathrm{m}^{2} / \mathrm{ms}$. 


\subsection{Supplement}

\subsubsection{Note: homotypic fusion rate gradient from the active zone}

To explore the possible homotypic fusion rate profiles from the active zone, we first calculated the $\mathrm{Ca}^{2+}$ profiles resulting from different $\mathrm{Ca}^{2+}$ currents strength and topology configurations, different $\mathrm{Ca}^{2+}$ buffering. Then using two release models (Schneggenburger and Neher, 2000; Beutner et al., 2001), we calculated the possible homotypic fusion rates at different distances from the active zone.

$\mathrm{A} \mathrm{Ca}^{2+}$ current entered through a disk of radius $\mathrm{R}$ situated in the $x-y$ plasma membrane plane, perpendicular to the $z$-direction. The calcium concentration $\left[\mathrm{Ca}^{2+}\right]$ in cylindrical coordinates $\left(z, r=\sqrt{x^{2}+y^{2}}\right)$ was calculated at steady-state using the linear approximation (Naraghi and Neher, 1997) by integrating over the area of incoming current:

$$
[C a](r, z)=[C a]_{\infty}+\frac{2}{\pi R^{2}} \int_{0}^{\pi} d \theta \int_{0}^{R} d r^{\prime} r^{\prime} \delta[C a]\left(\sqrt{z^{2}+r^{\prime 2}+r^{2}-2 r r^{\prime} \cos (\theta)}\right)
$$

Where $\delta[C a](x)$ is the difference of the $\mathrm{Ca}^{2+}$ concentration to the background $\mathrm{Ca}^{2+}$ concentration at a distance $x$ from the center of a channel, calculated as in (Naraghi and Neher, 1997). No dependence on the angle $\theta$ is needed due to the cylindrical symmetry. From these concentrations we derived the mean release latency using either of the two release models (Schneggenburger and Neher, 2000; Beutner et al., 2001). We used the inverse of the latency as the mean release rate. The mean fusion rate profile perpendicular to the synaptic membrane was then fit by the following curve:

$$
f(z)=A \exp \left(-\left(z / \lambda_{z}\right)^{\alpha}\right)
$$

where $A\left(\mathrm{~ms}^{-1}\right)$ is the maximum fusion rate at $z=0 \mathrm{~nm}, \lambda_{Z}(\mathrm{~nm})$ is the characteristic decay range of the fusion rate, $\alpha$ is the power of the exponential decay, and $z(\mathrm{~nm})$ is the variable representing the distance from the synaptic membrane. The supplementary table 1 shows the results of this fit for different buffers $(0.5$ and 2 mM EGTA and $2 \mathrm{mM}$ BAPTA), $\mathrm{Ca}^{2+}$ currents $(1,8$ and $16 \mathrm{pA})$, and for when positioned either at the center $(r$ $=0 \mathrm{~nm})$ of a large $(\mathrm{R}=210 \mathrm{~nm}) \mathrm{Ca}^{2+}$ cluster or at the side $(r=70 \mathrm{~nm})$ of a smaller $\mathrm{Ca}^{2+}$ cluster $(R=50 \mathrm{~nm})$. We find that $\lambda_{z}$ varied from 4 to $360 \mathrm{~nm}$, and the power varied roughly between 1 and 2. For simulations of homotypic fusion we chose $\alpha=2$ to constrains homotypic fusion more close to the plasma membrane; we varied $\lambda_{\mathrm{Z}}$ as found by the fits. 
The supplementary Fig. 4.4 shows the $\mathrm{Ca}^{2+}$ concentration profile (a), the mean fusion latency profile (b) and the fusion rate profile for 2 example (c) of supplementary table 4.1.

\subsubsection{Supplementary Figures and Tables}
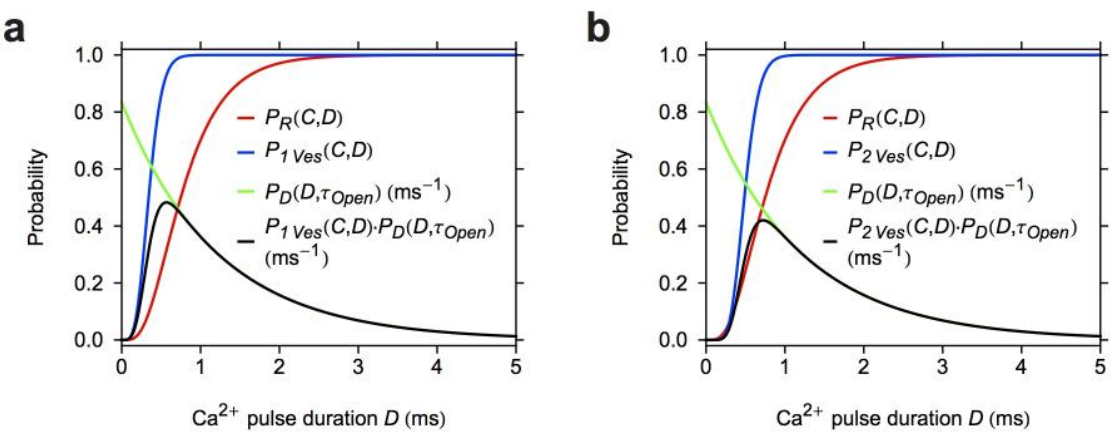

Supplementary Figure 4.1: Quantities used for the calculations of $\overline{N_{R}}$ and $\overline{T_{\text {asynch }}}$

(a) For the calculation of $\overline{N_{R}}\left(C, \tau_{\text {Open }}\right)$, where $C$ is the $\mathrm{Ca}^{2+}$ concentration and $\tau_{\text {Open }}$ is the ion channel mean open time. $D$ is the $\mathrm{Ca}^{2+}$ pulse duration.

Red: vesicular release probability $P_{R}(C, D)$. Blue: probability that at least 1 vesicle is released as a result of the Ca pulse $P_{1 \text { ves }}(C, D)$, calculated with equation (4.10) from $P_{R}(C, D)$. Green: probability $P_{D}\left(D, \tau_{\text {Open }}\right)$ that the $\mathrm{Ca}^{2+}$ channel opens for a duration $D$. Black: $P_{1 \text { ves }}(C, D) \cdot P_{D}(D$, $\left.\tau_{\text {Open }}\right)$ used in equation (4.8) for the calculation of $\overline{N_{R}}\left(C, \tau_{\text {Open }}\right)$.

(b) For the calculation of $\overline{T_{\text {asynch }}}\left(C, \tau_{\text {Open }}\right)$,

Red: vesicular release probability $P_{R}(C, D)$. Blue: probability of at least 2 vesicles get released $P_{2 \text { Ves }}(C, D)$, calculated with equation (4.11) from $P_{R}(C, D)$. Green: probability $P_{D}\left(D, \tau_{\text {Open }}\right)$ that the $\mathrm{Ca}^{2+}$ channel opens for a duration $D$. Black: $P_{E}(C, D) \cdot P_{2 v e s}\left(D, \tau_{\text {Open }}\right)$ used in equation (4.9) for the calculation of $\overline{T_{\text {asynch }}}\left(C, \tau_{\text {Open }}\right)$.

Parameters for this Figure: $\tau_{\text {Open }}=1.2 \mathrm{~ms}, C=120 \mu \mathrm{M}$, number of vesicles $N_{A}$ sensing the pulse: 7, (Beutner et al., 2001) secretion model with $\gamma=10 \mathrm{~ms}^{-1}$. 
$\mathrm{Ca}^{2+}$-triggered coordinated exocytosis model of MQR

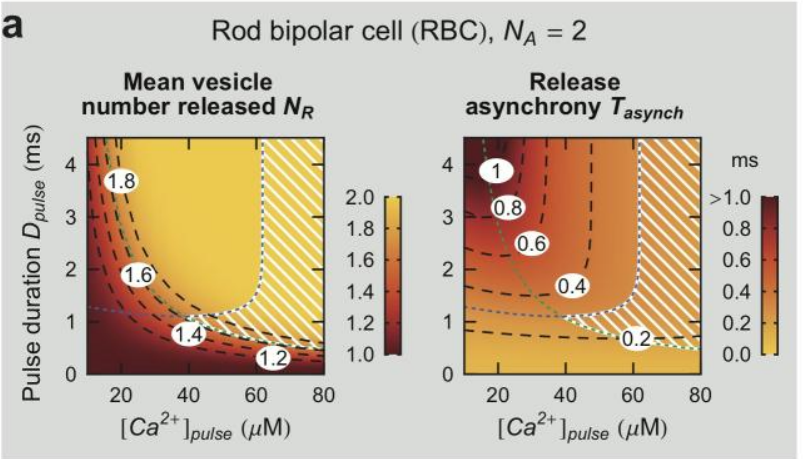

b Bullfrog amphibian papilla hair cell (frog $\mathrm{HC}$ ), $N_{A}=7$
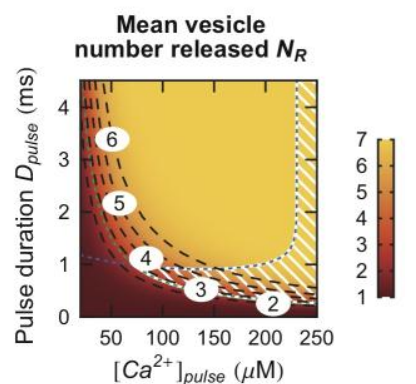

Release asynchrony $T_{\text {asynch }}$
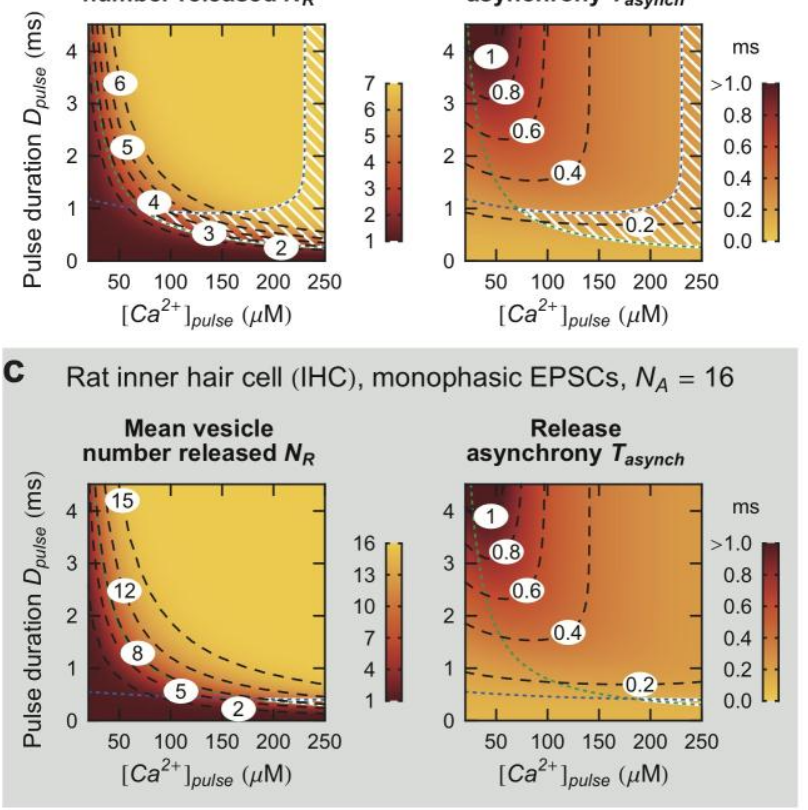

Supplementary Figure 4.2: Screening the temporal precision and efficiency of $\mathrm{Ca}^{2+}$ coordinated release model of MQR in terms of $\left[\mathrm{Ca}^{2+}\right]_{p u l s e}$ and Dpulse.

We calculated for which combination of pulse duration $D_{\text {pulse }}$ and concentration $\left[\mathrm{Ca}^{2+}\right]_{\text {pulse }}$ the $\mathrm{Ca}^{2+}$ coordinated release model is consistent with experimental observation (white stripes, consistency region in the parameter space, Table 4.1) in terms of number of vesicles released per release event $N_{R}$ and of the release asynchrony $T_{\text {asynch }}$ for ranges of $\mathrm{Ca}^{2+}$ concentrations $\left[\mathrm{Ca}^{2+}\right]_{\text {pulse }}$ (up to $250 \mu \mathrm{M})$ and durations $D_{\text {pulse }}(0$ to $4.5 \mathrm{~ms}$ ) potentially resulting from the opening of a single $\mathrm{Ca}^{2+}$ channel. The blue and green dashed lines result from the criteria on the synchrony and the mean number of vesicles released, respectively. The mean number of vesicles released $N_{R}$ increased with $\left[\mathrm{Ca}^{2+}\right]_{\text {pulse }}$ and $D_{\text {pulse. }}$. The region where $N_{R} \approx N_{A}$ (the number of vesicles feeling the $\mathrm{Ca}^{2+}$ pulse) corresponds to where the probability of release $P_{R} \approx 1$. In this area the release asynchrony $T_{\text {asynch }}$ only decreased with increasing $\left[\mathrm{Ca}^{2+}\right]_{\text {pulse }}$. In the region where $P_{R}<1, T_{\text {asynch }}$ mainly decreased with $D_{\text {pulse }}$. Each row is the $\mathrm{Ca}^{2+}$ coordinated release model applied to a specific system, assuming a particular number of available vesicles $N_{A}$ (Table 4.1):

(a) The consistency region was extended. It began at around $\left[\mathrm{Ca}^{2+}\right]_{\text {pulse }}=40 \mu \mathrm{M}$ for a small range of durations around $D_{\text {pulse }}=1 \mathrm{~ms}$, and at about $60 \mathrm{M}$, all durations above $0.5 \mathrm{~ms}$ were included.

Using (Schneggenburger and Neher, 2000) secretion model with final release step $\gamma=10 \mathrm{~ms}^{-1}$.

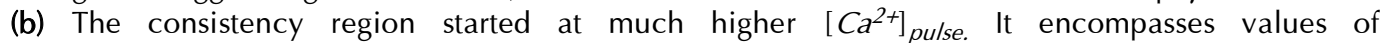
$\left[\mathrm{Ca}^{2+}\right]_{\text {pulse }}$ from around $75 \mathrm{M}$ and $D_{\text {pulse }}$ values between 0.5 and $1 \mathrm{~ms}$. Only from $\left[\mathrm{Ca}^{2+}\right]_{\text {pulse }}>$ $230 \mu \mathrm{M}$, all durations above $0.25 \mathrm{~ms}$ were included.

Using (Beutner et al., 2001) secretion model with $\gamma=10 \mathrm{~ms}^{-1}$.

(c) A very thin consistency region appears above $200 \mu \mathrm{M}$.

Using (Beutner et al., 2001) secretion model with $\gamma=10 \mathrm{~ms}^{-1}$. 
a

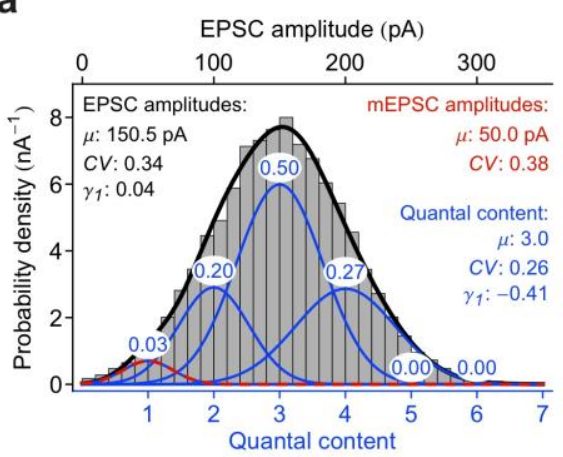

b

RBC:

quantal content: binomial distribution with $n=2, p=0.78$ mean 1.64

CV 0.29

skewness $=-0.58$ c Frog HC:

EPSC distribution: gaussian with mean $129+/-24$ pA CV 0.35 skewness $\sim 0$ d

IHC:

superposed on EPSC

distribution: gaussian $390+/-120$ pA

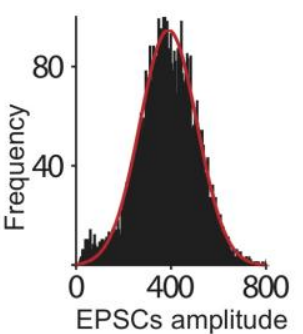

e

Frog HC: fitting a gaussian of mean 150 pA and s.d. 52 pA
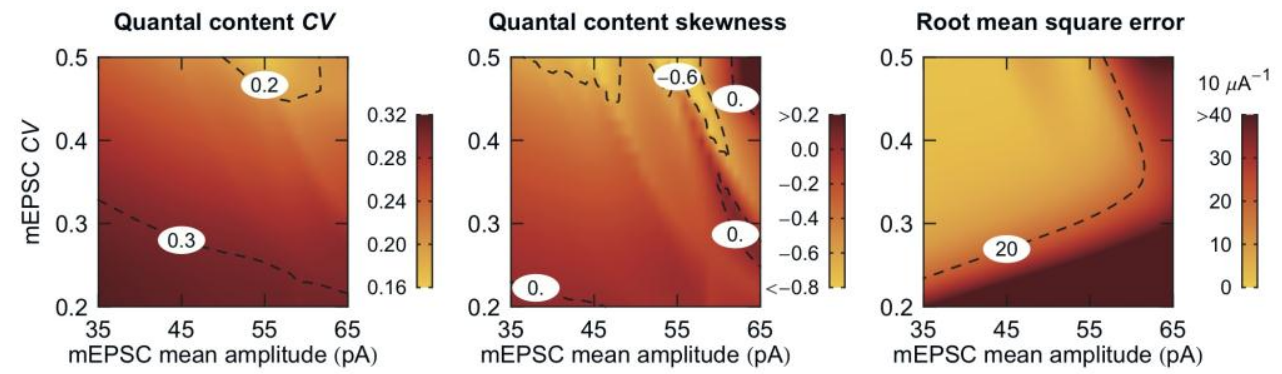

IHC: fitting a gaussian of mean 390 pA and s.d. 120 pA
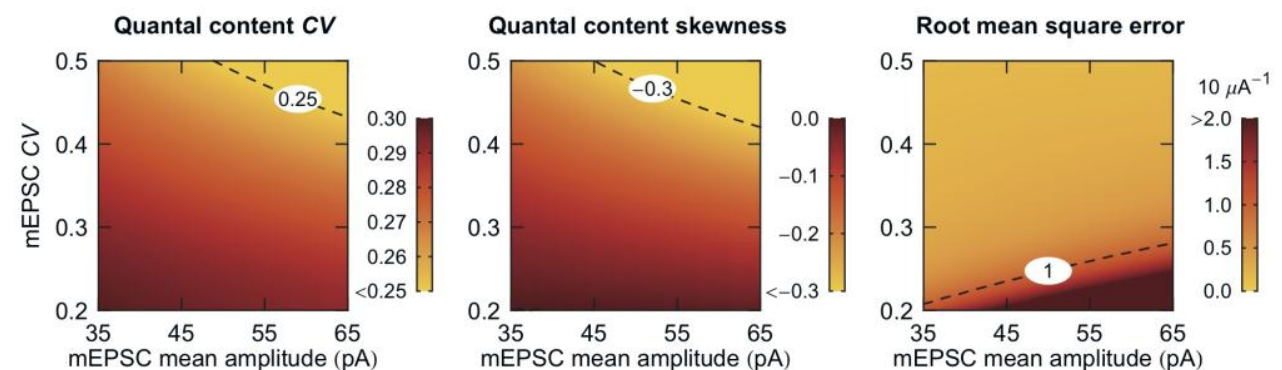

\section{Supplementary Figure 4.3: Quantal content distributions underlying the EPSC distributions.}

(a) Artificially generated EPSC amplitude histogram with amplitudes drawn from a Gaussian distribution with mean of $150 \mathrm{pA}$ and s.d. $=52$ pA. 20000 EPSCs were generated from which all EPSCs with negative amplitude were discarded. The characteristics of the resulting histogram of ESPC amplitudes are shown in black. The coefficient of variation $C V$ is 0.34 and the skewness $\gamma_{1}$ is close to 0 (0.04). The cumulative distribution of the amplitudes was fitted by a sum of 6 Gaussians with means $=i * 50 \mathrm{pA}$ and s.d. $=\operatorname{Sqrt}(I) * 19 \mathrm{pA}$ with $i$ ranging from 1 to 6 . The composing distributions are shown in blue, the mini amplitude distribution is shown in red dashed (mean $50 \mathrm{pA}$ and $C V$ 0.38). The numbers at the top of each distribution are their relative contribution. The quantal content is the number of neurotransmitter quanta an EPSC is made of. In blue are the characteristics of the quantal content distribution. Note the negative skewness $\gamma_{1}$, regardless of the fact the EPSC amplitude distribution had a skewness $\gamma_{1}$ of $\sim 0$.

(b) Characteristics of the quantal content distribution from the rat rod bipolar cell (RBC) as found by (Li et al., 2009) during strong stimulation. Mean, CV and skewness calculated neglecting the no-fusion events using the binomial distribution; 
(c) Characteristics of the EPSC distribution from the bullfrog amphibian papilla hair cells derived from ( $\mathrm{Li}$ et al., 2009)

(d) Characteristics of the EPSC distribution of ANF fiber with mainly monophasic EPSC from the IHC. Gaussian distribution superimposed onto EPSC amplitude of fibers with mainly monophasic EPSCs. Adapted from (Grant et al., 2010).

(e) Characteristics of the possible quantal content distribution underlying the EPSC distribution of the frog $\mathrm{HC}$ of (c). Fitting done by a sum of Gaussian functions as in (a), using mEPSC distributions with different mean and $C V$. Where the error of the fitting is small, the CV covers the range from 0.16 to 0.32 whereas the skewness from -0.8 to 0.2 . The root means square error was obtained by integrating the squared difference between the EPSC Gaussian and the fit, then dividing it by $350 \mathrm{pA}$ (the largest EPSC size in frog $\mathrm{HC}$ ) and taking the square root.

(f) as (e), but on the EPSC distribution of the IHC (d). Where the error of the fitting is small, the $\mathrm{CV}$ covers the range from 0.2 to 0.3 whereas the skewness from -0.3 to 0 . The root means square error was obtained by integrating the squared difference between the EPSC Gaussian and the fit, then dividing it by $800 \mathrm{pA}$ (the largest EPSC size in IHC) and taking the square root.

The ranges for the $C V$ and for the skewness of the underlying quantal content distribution for the frog $\mathrm{HC}$ were the widest, therefore we chose these ranges as limits of experimental quantal content distributions: $0.16<C V<0.32,-0.8<$ skewness $<0.2$. 


\begin{tabular}{|c|c|c|c|c|c|c|}
\hline \multirow[b]{2}{*}{ Configuration } & \multirow[b]{2}{*}{$\begin{array}{c}\text { Buffer } \\
\text { (mM) }\end{array}$} & \multirow[b]{2}{*}{$\begin{array}{c}\text { Total } \\
\text { current } \\
\text { (pA) }\end{array}$} & \multicolumn{2}{|c|}{$\begin{array}{c}\text { Beutner model of } \\
\text { secretion }\end{array}$} & \multicolumn{2}{|c|}{$\begin{array}{l}\text { Schneggenburger } \\
\text { model of secretion }\end{array}$} \\
\hline & & & $\lambda_{z}(\mathrm{~nm})$ & $\alpha$ & $\lambda_{z}(\mathrm{~nm})$ & $\alpha$ \\
\hline \multirow{9}{*}{$\begin{array}{l}\mathrm{Ca}^{2+} \quad \text { current } \\
\text { flowing through a } \\
\text { disk with a radius } \\
\mathrm{R} \text { of } 50 \mathrm{~nm}, \mathrm{z} \\
\text { fusion rate profile } \\
\text { at } \mathrm{r}=70 \mathrm{~nm} \text { from } \\
\text { the center of the } \\
\text { disk. }\end{array}$} & \multirow{3}{*}{$\begin{array}{l}\text { EGTA } \\
0.5\end{array}$} & 1 & 89 & 1.3 & 109 & 1.3 \\
\hline & & 8 & 146 & 1 & 254 & 1.2 \\
\hline & & 16 & 178 & 1 & 353 & 1.3 \\
\hline & \multirow{3}{*}{$\begin{array}{l}\text { EGTA } \\
2\end{array}$} & 1 & 75 & 1.5 & 91 & 1.5 \\
\hline & & 8 & 122 & 1.2 & 191 & 1.4 \\
\hline & & 16 & 144 & 1.2 & 252 & 1.6 \\
\hline & \multirow{3}{*}{$\begin{array}{l}\text { BAPTA } \\
2\end{array}$} & 1 & 14 & 1.8 & 15 & 1.8 \\
\hline & & 8 & 23 & 2.1 & 27 & 2.1 \\
\hline & & 16 & 28 & 2 & 33 & 2.1 \\
\hline \multirow{9}{*}{$\begin{array}{l}\mathrm{Ca}^{2+} \text { current } \\
\text { flowing through a } \\
\text { disk with a radius } \\
\mathrm{R} \text { of } 210 \mathrm{~nm}, \mathrm{z} \\
\text { fusion rate profile } \\
\text { in the center of } \\
\text { the disk } \\
(\mathrm{r}=0 \mathrm{~nm})\end{array}$} & \multirow{3}{*}{$\begin{array}{l}\text { EGTA } \\
0.5\end{array}$} & 1 & 92 & 1.2 & 117 & 1.3 \\
\hline & & 8 & 184 & 1.1 & 278 & 1.3 \\
\hline & & 16 & 217 & 1.1 & 371 & 1.4 \\
\hline & \multirow{3}{*}{$\begin{array}{l}\text { EGTA } \\
2\end{array}$} & 1 & 67 & 1.3 & 85 & 1.3 \\
\hline & & 8 & 140 & 1.2 & 197 & 1.4 \\
\hline & & 16 & 162 & 1.2 & 254 & 1.5 \\
\hline & \multirow{3}{*}{$\begin{array}{l}\text { BAPTA } \\
2\end{array}$} & 1 & 4 & 1 & 5 & 1 \\
\hline & & 8 & 10 & 1.4 & 13 & 1.5 \\
\hline & & 16 & 14 & 1.5 & 17 & 1.5 \\
\hline
\end{tabular}

Supplementary Table 4.1: Fit to fusion rate profile perpendicular from the active zone in different configurations.

a

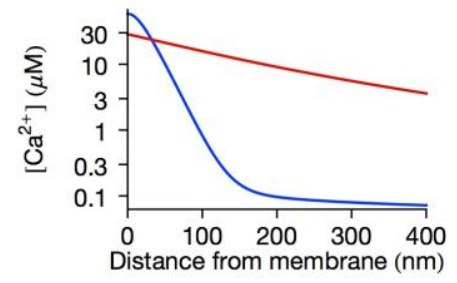

b

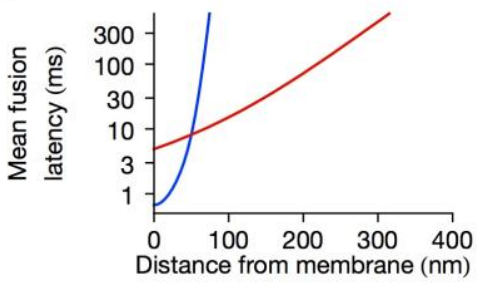

C

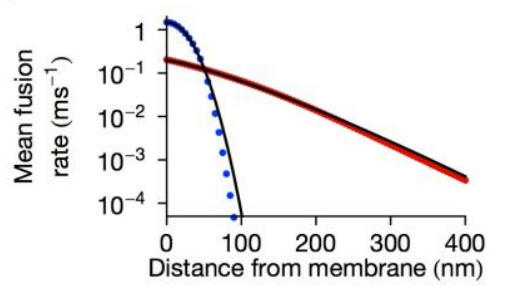

Supplementary Figure 4.4: Fusion rates profiles at the active zone.

Red: parameters in red from supplementary table 1 ;

Blue: parameters in blue from supplementary table 1 ;

(a) $\mathrm{Ca}^{2+}$ profile as a function of the distance $z$ from the active zone.

(b) Mean latency as a function of the distance $z$ from the active zone.

(c) Fusion rate as a function of the distance $z$ from the active zone, calculated as the inverse

of the mean latency from (b). Black: fits using the function (4.24) of the supplementary note. 
a $\mathrm{Ca}^{2+}$-dependent homotypic fusion model of MQR, 1D top-down vesicle track, $\phi_{1 D}=50 \%$
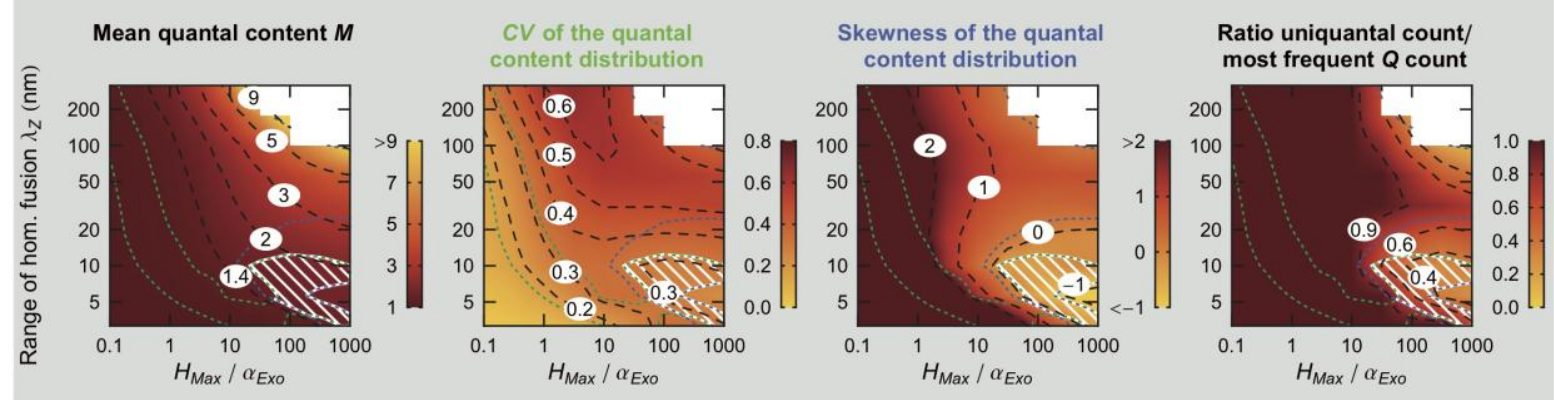

b

$\mathrm{Ca}^{2+}$-dependent homotypic fusion model of MQR, vesicles on the 2D ribbon surface, $\phi_{2 D}=25 \%$
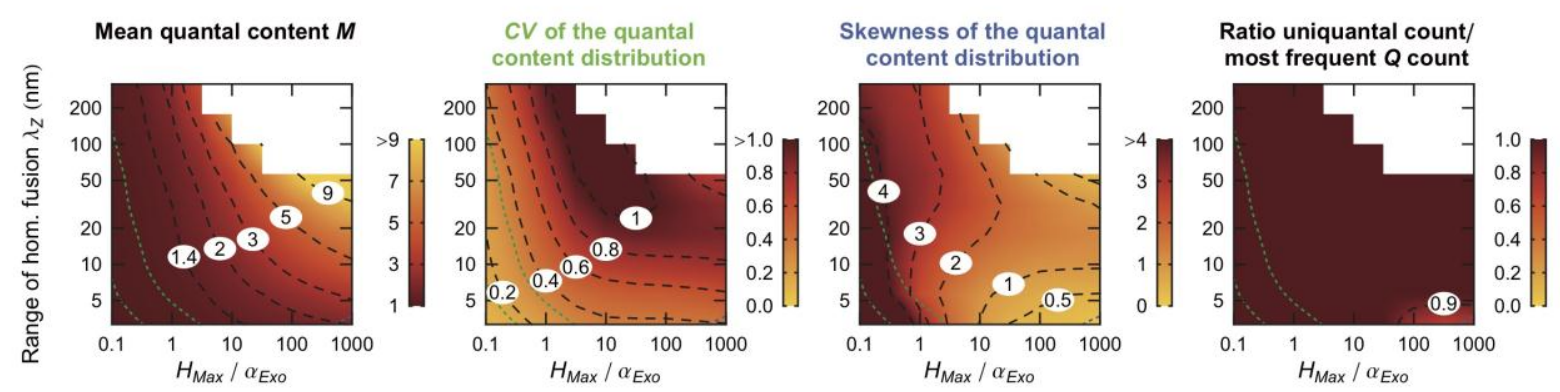

Vesicle quantal content and $\mathrm{Ca}^{2+}$-dependent homotypic fusion model of $\mathrm{MQR}$, vesicles on the $2 \mathrm{D}$ ribbon surface, $\phi_{2 D}=25 \%$
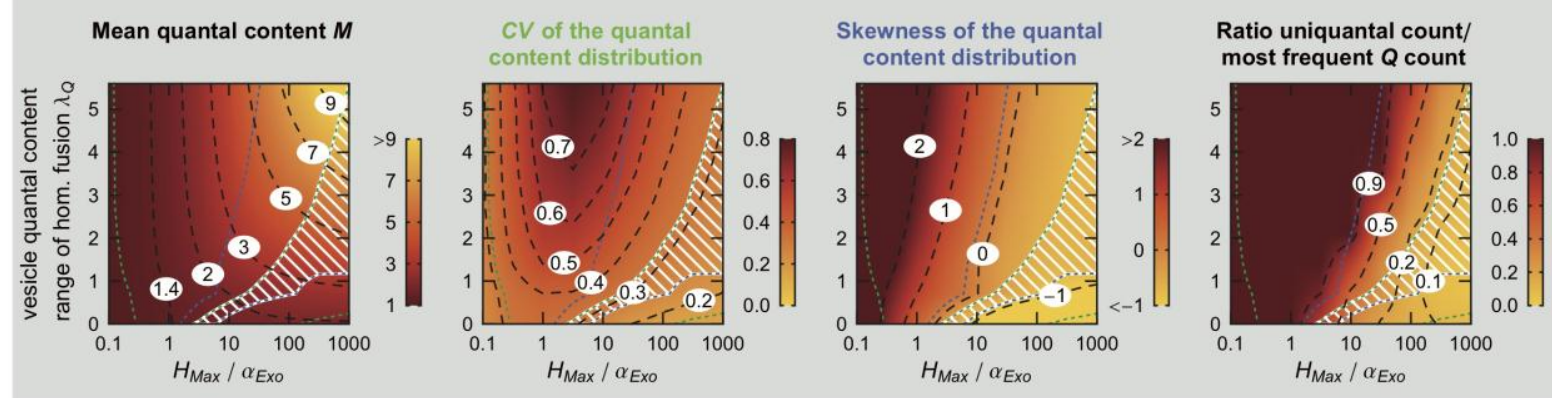

Supplementary Figure 4.5: Homotypic fusion models for lower packing densities

(a-c) Same as Fig. 4.5e,f, Fig. 4.6e but with vesicle packing densities $\varphi_{1 D}$ and $\varphi_{2 D}$ at 0.5 and 0.25 , respectively. The consistency regions shifted to higher values of $H_{\text {Max }} / \alpha_{\text {exol }}$ consistent with the fact that a lower concentration enables less homotypic fusion.

In the $2 \mathrm{D} \mathrm{Ca}^{2+}$-dependent model of homotypic fusion, no consistency region was present. 
a $\mathrm{Ca}^{2+}$-dependent homotypic fusion model of MQR, 1D top-down vesicle track, $\phi_{1 D}=90 \%$
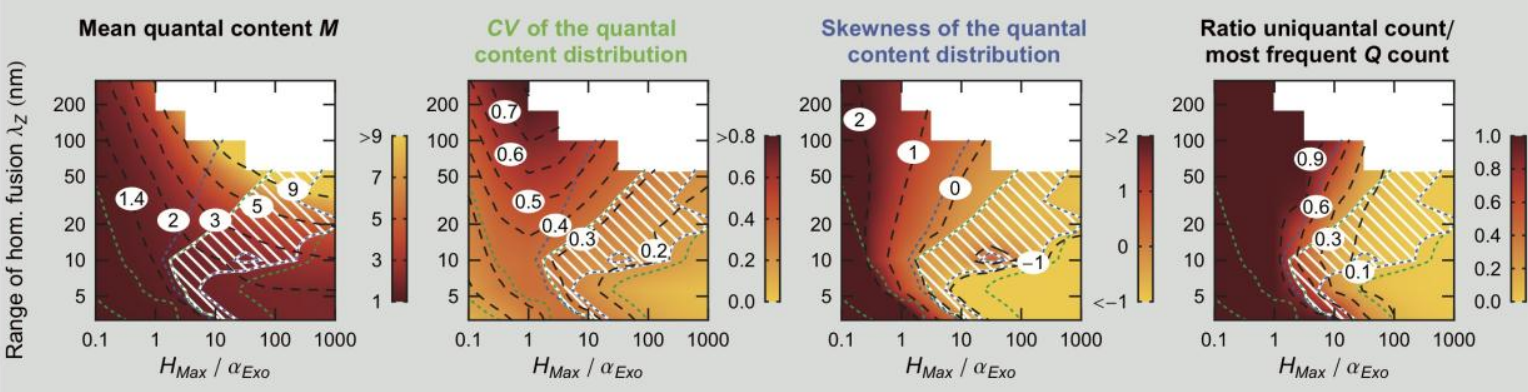

b

$\mathrm{Ca}^{2+}$-dependent homotypic fusion model of MQR, vesicles on the $2 \mathrm{D}$ ribbon surface, $\phi_{2 D}=55 \%$
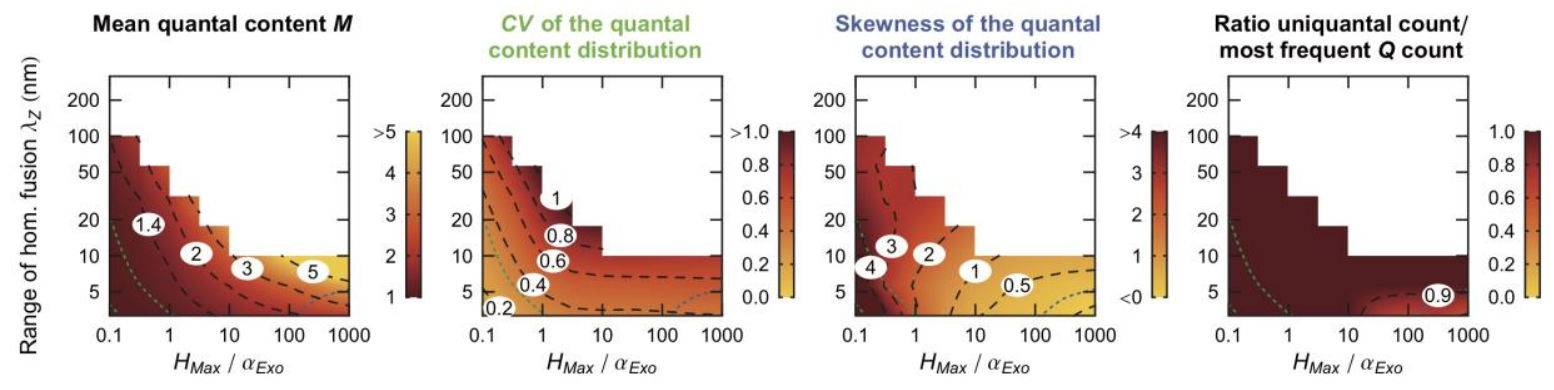

Vesicle quantal content and $\mathrm{Ca}^{2+}$-dependent homotypic fusion model of MQR, vesicles on the 2D ribbon surface, $\phi_{2 D}=55 \%$
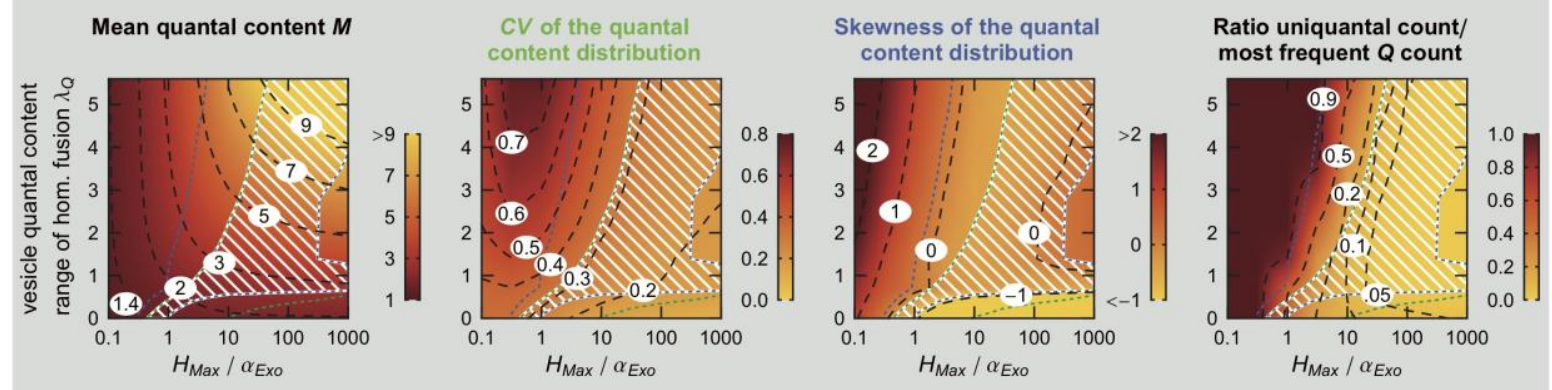

\section{Supplementary Figure 4.6: Homotypic fusion models for higher packing densities}

(a-c) Same as Fig. 4.5e,f, Fig. 4.6e but with vesicle packing densities $\varphi_{1 D}$ and $\varphi_{2 D}$ at 0.9 and 0.55 , respectively. The consistency regions shifted to lower values of $H_{\text {Max }} / \alpha_{e x O}$ consistent with the fact that a higher concentration enables more homotypic fusion.

In the 2D Ca ${ }^{2+}$-dependent model of homotypic fusion, no consistency region was present. 



\section{Uniquantal and frustrated release at the inner hair cell ribbon synapse}

All experimental data in this study was provided by Darina Khimich.

\section{Short summary}

In chapter 4 we suggested that multiquantal release was not a plausible interpretation of large EPSCs triggered by neurotransmitter release from IHCs. We showed that an alternative plausible explanation could be that a single vesicle fusion generates large EPSCs and that vesicle fusion pore flickering cause multiphasic EPSCs. In this chapter, we analyzed post-synaptic bouton recordings from the spiral ganglion neurons. In particular we investigated whether multiphasic EPSCs could or could not be decomposed into mEPSC. 


\subsection{Introduction}

Neurotransmitter release from the inner hair cell (IHC) synapse generates spiking in auditory nerve fibers with high reliability (Chapter 2, (Siegel, 1992)). This reliability was assigned to the large excitatory postsynaptic current (EPSC) amplitudes (300-400 pA on average) (Grant et al., 2010). It has been always assumed that these large EPSCs arise from the synchronous fusion of many (up to 16) vesicles, or the fusion of large compounds (Glowatzki and Fuchs, 2002; Matthews and Fuchs, 2010). This multiquantal release interpretation rose in part from the observation of EPSC waveforms vary greatly in shape. Some EPSCs are monophasic - with a single rise time, whereas others are multiphasic - resembling the superposition of multiple miniature EPSCs (mEPSCs). Whether these EPSC shapes really result from the fusion of many vesicles has now been questioned (Chapter 4). In fact, we proposed that the large EPSCs might result from the exocytosis of just a single vesicle, whereas the multiphasic EPSCs emerge from vesicle pore flickering. The prediction of this hypothesis is that multiphasic EPSC shapes are not readily interpretable as the superposition of multiple desynchronized mEPSCs.

In this study we analyzed postsynaptic voltage clamp recordings from the bouton of auditory nerve fibers, attached to the afferent ribbon synapse of the IHC. First we propose a method to easily differentiate between multiphasic and monophasic EPSCs. We found that the average charge of multiphasic EPSCs is similar to that of monophasic EPSCs, indicating that multiphasic and monophasic events result from on average the release of the same amount of neurotransmitter. Then, using deconvolution, we decomposed multiphasic EPSCs into a superposition of mEPSCs. By doing so, we found that all sizes down to the noise level of underlying "mEPSCs" would be required to compose the shapes of multiphasic EPSCs. We propose that the variable EPSC shapes are more readily explained by the fusion of a single vesicle with a flickering fusion pore. In conclusion our results suggest that the uniquantal interpretation is a plausible alternative to the classical multiquantal release interpretation of the electrophysiological data acquired from the IHC ribbon synapses afferences. 


\subsection{Results}

\subsubsection{Amplitude and charge of monophasic and multiphasic EPSCs}

Voltage clamping of the spiral ganglion neuron afferent boutons exhibited abundant synaptic activity driven by the IHC (Fig. 5.1a-b). To characterize EPSC shapes, we studied their amplitude and charge distributions (Methods). The EPSC amplitude indicates the maximum open probability of AMPA receptors (AMPAR) reached during the EPSC time course. The EPSC charge, however, is linearly correlated to the amount of neurotransmitter that was released in a particular release event, provided the absence of AMPAR saturation or desensitization. The mean amplitude and charge among cells were $260 \pm 160 \mathrm{pA}$ and $450 \pm 280 \mathrm{fC}$, respectively $(\mathrm{n}=3)$. We found that for each cell the coefficient of variation $(C V=$ s.d. / mean) of the charge distribution $(C V=0.34, \mathrm{n}=3)$ was smaller than the $C V$ of the amplitude distribution $(C V=0.42, \mathrm{n}=3)$ (Fig. 5.1c-d), although this difference was not significant $(\mathrm{p}=0.22)$. Overall, the charge distribution was more symmetrical and Gaussian-like than the amplitude distribution. Although one of the amplitude distributions revealed a peak around $50 \mathrm{pA}$ and a tail at higher amplitudes, all the charge distributions were symmetrical and did not reveal any peak at small charges. This suggests that the peak at small amplitudes that was usually interpreted as "uniquantal", exists only in the amplitude distribution of the EPSCs (Glowatzki and Fuchs, 2002) and does not correlate with an uniquantal amount of neurotransmitter, as no small charge peak was present in the charge distribution. In summary, the variability of the neurotransmitter content and its distribution was consistent with the one expected for a single vesicle (del Castillo and Katz, 1954).

To better understand the origin of the slight difference in amplitude and charge distributions, we plotted the dependence of EPSC charge on EPSC amplitude in a scatter plot (Fig. 5.1e). The resulting map is very different from the one of shown by (Rossi et al., 1994), where all the points were aligned on the diagonal. Only the upper left triangle is filled with data points. This indicates that an EPSC of given amplitude must have a certain minimal charge. This is a consequence of the fixed decay time constant of the AMPA receptors open probability, which restricts smaller charges for one given amplitude. 
a

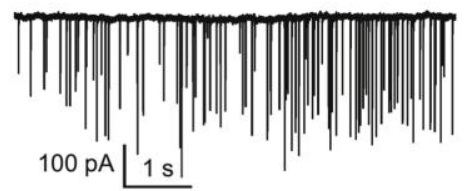

C

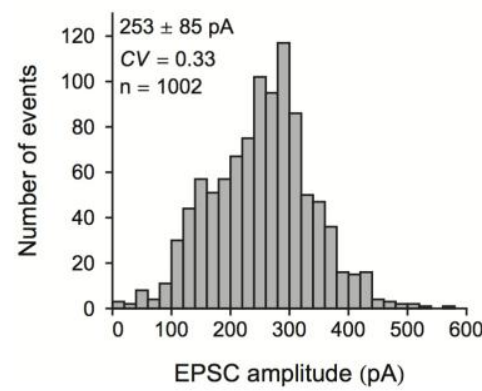

e

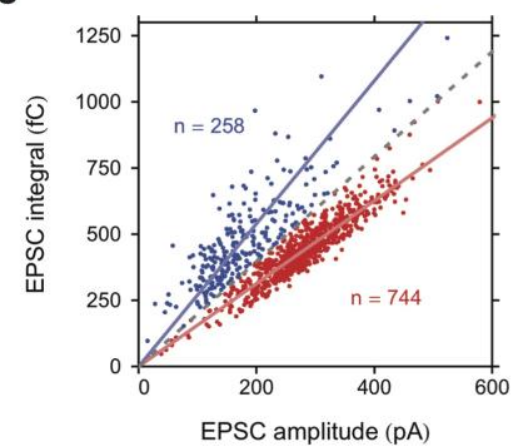

g

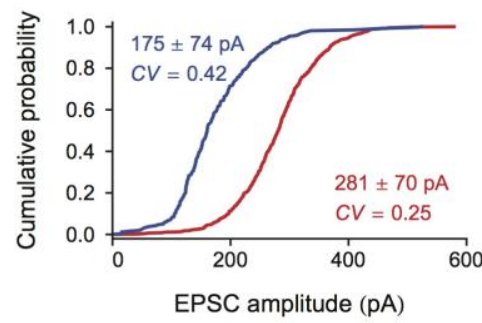

b

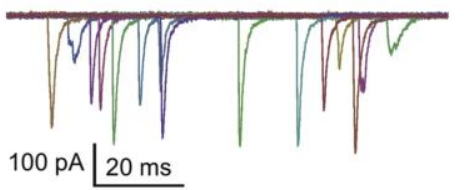

d

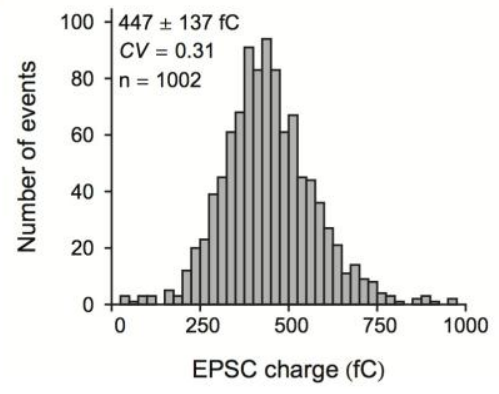

f

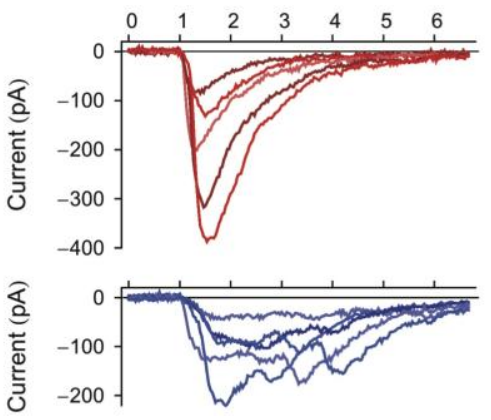

h

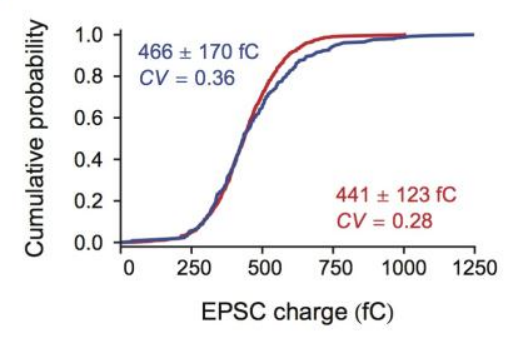

Figure 5.1: EPSC amplitude and charge for monophasic and multiphasic EPSCs.

(a) Example of a current trace during abundant spontaneous activity. (b) Superposed 15 consecutive $100 \mathrm{~ms}$ of a voltage clamp recording. One can distinguish EPSCs of different shapes: fast monophasic EPSCs and slower multiphasic EPSCs.

(c) Distribution of EPSC amplitudes revealed two bumps.

(d) The distribution of EPSC charges was rather symmetric.

(e) Scatter plot of EPSC charge versus charge. Two clouds of points are distinguishable. One (red) are on the diagonal, the other (blue) are separated by the gray dashed line. More monophasic EPSCs were present in this cell.

(f) Examples of events taken from the two clouds. The red ones are all rather fast and monophasic. The blue are slow and more extended in time.

(g) Cumulative distribution of the EPSC amplitudes for both groups. Multiphasic EPSC had significantly smaller amplitude in all cells tested.

(h) Cumulative distribution of the EPSC charges for both groups. No significant difference was seen among the cells tested. Here one sees few EPSC with larger charge in the multiphasic group. This could be explained by stochastic superposition of independent EPSCs. 
In all cells $(\mathrm{n}=3)$ we found two higher density spots in the amplitude/charge scatter plots. One high density spot lied on the diagonal, where EPSCs have the smallest ratio of charge over amplitude (red, Fig. 5.1e). These are the fastest EPSCs - with the fastest rise time, as the EPSC decay time is rather stereotypical. These can be assimilated to the usual monophasic EPSCs (Fig. 5.1f). A second distinct cloud of points was on the left of the diagonal (blue, Fig. 5.1e). This represented EPSCs with a less "optimal" charge usage: they were smaller, and with larger full width at half-maximum (FWHM). These points were assimilated to the multiphasic EPSCs (Fig. 5.1g).

Although there were always some points present between these two high density spots, one could well separate them into two groups with a line passing through the point of $0 \mathrm{fC}$ charge and $0 \mathrm{pA}$ amplitude (gray dashed line Fig. 5.1e). EPSCs on the right side of the line were called monophasic, whereas those on the left side multiphasic. In one cell we found multiphasic EPSCs with a charge expected from for a "uniquantal" (50 pA) EPSC. Such an observation would not suit the multiquantal release interpretation of multiphasic EPSCs, as one would need to assume that a single vesicle could be desynchronized. In contrast, pore flickering could account for multiphasic EPSCs with a small charge.

Next we studied the amplitude and charge distribution of monophasic and multiphasic EPSCs independently. The mean amplitude of monophasic EPSCs was significantly larger than the one of the multiphasic in each cell $(n=3)$. However the mean charge distribution was not significantly different. (Keen and Hudspeth, 2006) reported that in their preparation of frog amphibian papilla hair cell afference, multiphasic EPSCs were on average larger than the monophasic ones. This is compatible with a linear superposition of independent EPSCs. Here, this was not the case: indicating that monophasic EPSCs were not just a superposition of independent release events.

\subsection{2 mEPSCs do not compose multiphasic EPSCs}

What is the underlying temporal structure of neurotransmitter release in multiphasic EPSCs? If it was the release of independent vesicles that would cause the multiphasic shape of EPSC, then neurotransmitter release would occur at definite distinct time points with a stereotypical amplitude. However, if it is was continuous release through a flickering fusion pore, then neurotransmitter might be released in a less stereotypical fashion and all sizes of underlying events could be expected. 
a

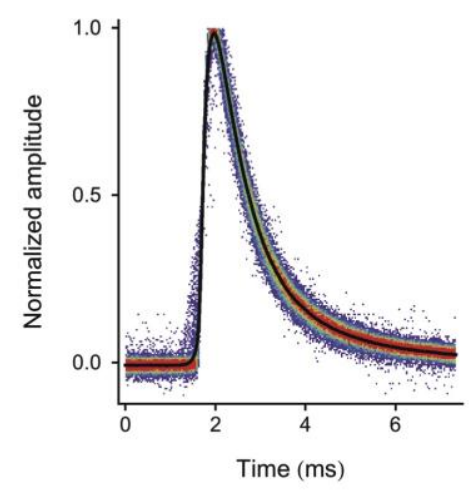

b

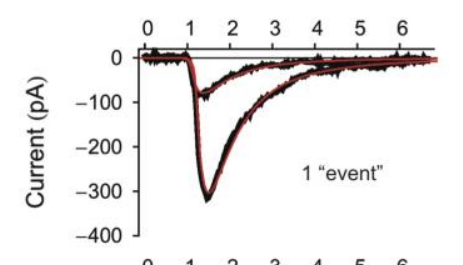

Time (ms)
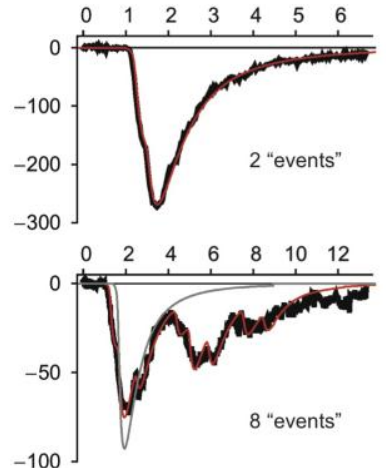

C

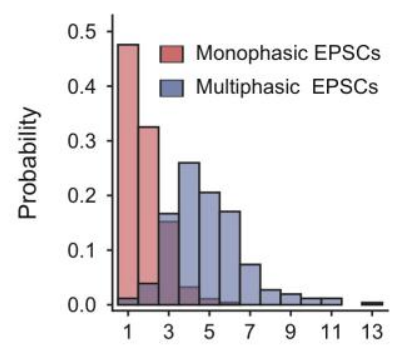

Number of underlying "events" d

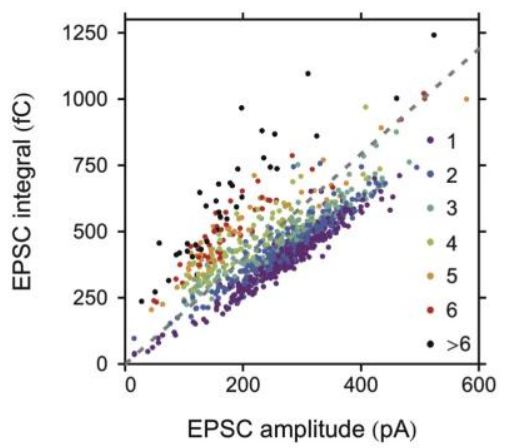

e

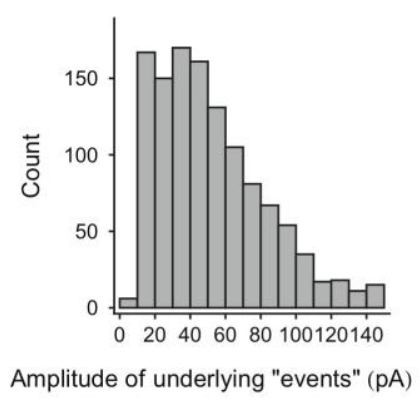

Figure 5.2: Decomposition of EPSCs into elementary events

(a) By taking all the events at the right of the red line for Fig. 1e, normalizing, aligning them at the half rise time and averaging we obtain the template EPSC (black). Color pseudo-scale of the density of all the superimposed events (395 EPSCs)

(b) Examples of the fit done by the iterative deconvolution algorithm. In red is the fit with $1,2,5$ and 8 underlying events. For the 1 , there are 2 examples. In gray are the $1^{\text {st }}$ fit done during the iterative decomposition to have a comparison of time scales. For the 8 events case, note the twice slower time scale.

(c) Number of underlying events given by the decomposition. Monophasic EPSCs are decomposed by on average $\sim 2$ events, whereas the multiphasic EPSCs by on average 5 .

(d) Same scatter plot as in Fig. 5.1e, but with coloring indicating the number of underlying events. This suggests that this representation can well distinguish between different types of EPSCs.

(e) By taking all the EPSCs with at least 3 underlying release events, we plotted the amplitude distribution of all these underlying events. There is no quantal peak at $50 \mathrm{pA}$, EPSC of very small sizes are needed to describe the shapes of EPSCs (see 8 events shapes in (b)) 
We tested whether multiphasic EPSCs could be composed of distinct vesicle releases. First we constructed the "ideal" monophasic EPSC that would result from the exocytosis of a single vesicle. We took the fastest monophasic EPSC shapes, normalized, aligned and averaged them (Fig. 5.2a, Methods). Then, using an iterative fitting/deconvolution algorithm, we investigated how many "ideal" EPSCs compose the multiphasic EPSCs (Fig. 5.2b). Whereas most monophasic EPSCs were decomposed by on average in 2 "ideal" EPSCs, the multiphasic EPSCs required much more (on average 5) (Fig. 5.2c). We plotted this information on the scatter plot (Fig. 5.2d). The good separation of the events in this representation suggests that this is an appropriate way to distinguish monophasic EPSCs from multiphasic ones, which was done visually event by event previously (Glowatzki and Fuchs, 2002; Grant et al., 2010).

Finally we looked at the amplitude distribution of the underlying events by talking all the EPSCs that were composed of at least 3 events (Fig. 5.2f). In all of the cells ( $n=3$ ), we observed that the contributions of all amplitudes down to the noise level of underlying "ideal" EPSCs would be required to account for the shapes of multiphasic EPSCs. This fact is in contrast to what is expected if multiphasic EPSCs resulted from the desynchronized release of $50 \mathrm{pA}$ vesicles. This additional evidence is consistent with the picture of a vesicle fusion pore flickering that can open and close for different durations and with different diameters, and let higher or lower amounts of neurotransmitter escape into the synaptic cleft. 


\subsection{Discussion}

In this study we examined the nature of the multiphasic EPSCs from the IHC ribbon synapses. These EPSCs were usually interpreted as the superposition of desynchronized vesicles (Glowatzki and Fuchs, 2002; Grant et al., 2010) and led to the now accepted multiquantal release interpretation of EPSCs in the ribbon synapse field (Matthews and Fuchs, 2010). We found that in the charge distribution of EPSC, there was no sign of a uniquantal peak at a charge smaller than the average EPSC charge. We found that multiphasic and monophasic EPSCs had on average the same neurotransmitter content. And finally our results suggest that multiphasic EPSCs are not readily deconvolved into a superposition of uniquantal EPSCs, but rather that all amounts of neurotransmitter release occur during a multiphasic EPSC.

These results would favor the uniquantal interpretation of EPSCs triggered by the IHC ribbon synapses. Multiphasic EPSCs would then be the consequence of a full vesicle fusion, but which occurs in several steps. Several short or small fusion pore opening would be required for the release of the neurotransmitter content of vesicles. This kind of pore flickering was observed at other synapses (Staal et al., 2004; Pawlu et al., 2004; Wang et al., 2008), but were not always interpreted as such (Girod et al., 1993). In addition pore flickering were observed at other secretion cells but usually with larger vesicles and at longer time scales (Zhou et al., 1996; Chanturiya et al., 1997; Scepek et al., 1998; Takahashi et al., 2002). Whether this could be a sign of kiss-and-run type of exocytosis is not yet clear (Rizzoli and Jahn, 2007).

The uniquantal interpretation is consistent with many additional experimental findings at the synapse. (1) The EPSC distribution is independent of stimulation and of intracellular $\mathrm{Ca}^{2+}$ buffering (for example by changing the intracellular buffer from BAPTA to EGTA), whereas the release frequency is. (2) Known rates of exocytosis at the IHC and spike rates in the auditory nerve fibers (ANF) are more consistent in the uniquantal interpretation. In fact, steady state spike rates at the ANF are around $300 \mathrm{~Hz}$ (Taberner and Liberman, 2005). To trigger a spike, at least one EPSC is required (Chapter 2). If each EPSC is composed of on average 7 vesicles (Grant et al., 2010), one would need a vesicles release rate of at least $2100 \mathrm{~Hz}$ per synapses, although estimated rates are in the order $600 \mathrm{~Hz}$ per active zone (Pangrsic et al., 2010). (3) EM reconstruction of the IHC ribbon synapses reveal around 10 release sites, thus it seems unlikely to synchronize the release of 16 vesicles (Frank et al., 2010). Large compounds were rarely observed in EM imaging, also arguing against compound vesicle fusion. (4) 
The mEPSC amplitude increases linearly with the postsynaptic density (PSD) area (Nusser et al., 1997). The fact that the PSD of ANFs (radius of around $400 \mathrm{~nm}$ ) is significantly larger than the PSD of cortical synapses, one would expect much larger mEPSCs in the ANFs.

Why would pore flickering be present at the IHC ribbon synapses? Multiphasic EPSCs seem to almost disappear with synapse maturation (Grant et al., 2010), which could be a sign of an immature exocytosis machinery. Finally, a single vesicle interpretation seems to be the most parsimonious interpretation in terms of energy consumption for the synapse: why release many vesicles at once if a single vesicle could give the same effect? 


\subsection{Methods}

Postsynaptic recording were performed at $-90 \mathrm{mV}$ holding potential as in (Glowatzki and Fuchs, 2002). Sampling frequency was $30 \mathrm{kHz}$. The analysis was done in 3 afferent recordings with abundant spontaneous activity driven by the IHC. In the graphs the analysis of one representative cell is shown. Analysis was performed in Mathematica (Wolfram Research).

The current traces were first high-pass filtered at $1 \mathrm{~Hz}$ to remove small baseline drifts and to enable easy detection of EPSCs. The recording was then shifted by $2-4 \mathrm{mV}$ so that the baseline of the recording was at $0 \mathrm{mV}$. The s.d. of the noise was usually around 3-4 pA. Some traces were low pass filtered at $10 \mathrm{kHz}$. EPSCs were detected using a threshold at $-15 \mathrm{pA}$. The beginning and the end of the EPSC were set as the point where the current trace crossed 0 pA. EPSC charge was calculated as the area between the beginning and the end of the EPSC. All events were inspected by eye.

Once the monophasic EPSCs were selected, they were normalized, aligned at the half of their rising phase and averaged. The result gave the "ideal" monophasic EPSC. For the averaging, the non low-pass data was used. This ideal monophasic EPSC was used as the point spread function (PSF) of the deconvolution. For convenience we set the time $t=0$ as the peak of the PSF, i.e., $\operatorname{PSF}(0)=1$.

The deconvolution algorithm of multiphasic EPSCs aimed at finding the minimum number of PSFs that could account for the EPSC shape. We wanted to minimize the error $E$ :

$$
E(n)=\int_{t_{\text {begin }}}^{t_{\text {end }}}(f(n, t)-\operatorname{EPSC}(t))^{2} d t
$$

where $\operatorname{EPSC}(t)$ is the EPSC shape, tbegin and $t_{\text {end }}$ are the beginning and the end of the EPSC, respectively. $f(n, t)$ was the fit function:

$$
f(n, t)=\sum_{i=1}^{n} A_{i} \cdot \operatorname{PSF}\left(t-t_{i}\right)
$$

where $t$ was time, $A_{i}$ and $t_{i}$ were the amplitudes and the times of underlying PSFs, respectively. PSF $(t)$ was the PSF and $n$ was the number of PSFs composing the fit function. We used an iterative approach to find the best fit. We fitted the EPSC by increasing the number $n$ of PSFs in the fit function $f(n, t)$. The algorithm was stopped when either of two criteria were met: the error $E(n)$ of the fit was smaller than 2.5 times 
the error expected from the noise or when increasing the number of PSFs didn't reduce the total error $E$ by more than $10 \%: E(n+1) / E(n)>0.9$.

For the fit at $n=1$, starting values for $A_{1}$ and $t_{1}$ where the amplitude and the peak time of the EPSC, respectively. For the fit at subsequent $n>1$, as starting values for $A_{i}$ and $t_{i}$ ( $i$ from 1 to $n-1$ ) were the values provided by the fit $n-1$, and the starting values for $A_{n}$ and $t_{n}$ were the amplitude and the time of the maximum error of the previous fit. 



\section{$6 \mathrm{Ca}^{2+}$ nanodomain control of exocytosis and its consequences on auditory nerve fiber response properties}

\section{Short summary}

In chapter 2 we showed that the postsynapse reliably encodes in spike trains the EPSCs triggered by the release of neurotransmitter from the inner hair cell ribbon synapse. If large EPSCs are in fact composed of one vesicle as suggested by chapters 4 and 5 , the exocytosis of single vesicle is sufficient to trigger an action potential in the auditory nerve fiber. In this final chapter we examine the functioning of $\mathrm{Ca}^{2+}$ channel nanodomain control of exocytosis and explore its consequences on the system level. In particular we investigate how it could account for different aspects of afferent auditory nerve fibers, like their response heterogeneity, long latencies at weak sound amplitudes and phase locking. 


\subsection{Introduction}

Redundancy in biological systems usually provides reliability. Surprisingly, at the birth of the auditory neural spike code, which is one of the most precise and fastest in the brain, vesicle release is controlled by only one or a few $\mathrm{Ca}^{2+}$ channels (Brandt et al., 2005). Here, using computational modeling, we explore the consequences and signatures of such a seemingly fragile design of neurotransmission at the inner hair cell (IHC) ribbon synapse.

The auditory system reliably encodes sound intensities spanning 6 orders of magnitude (from 0 to about 120 decibel (dB) sound pressure level (SPL)). Auditory nerve fibers (ANF), the first neurons in the auditory pathway, vary extensively in their spontaneous firing rate (rate in the absence of sound stimulus), ranging from 0 to about $100 \mathrm{~Hz}$ (Kiang, 1965; Liberman, 1978; Taberner and Liberman, 2005). Additionally, each ANF has its specific SPL threshold and range of intensities over which its spiking rate varies. Thus each ANF encodes only a fraction of the total intensity range covered by the auditory system. Finally, the spontaneous firing is negatively correlated to threshold SPL: high spontaneous rate fibers usually have low thresholds, whereas low spontaneous rate fibers have high thresholds. Despite major research efforts, the biophysical mechanisms underlying ANF response heterogeneity and the coupling between spontaneous rate and ANF sensitivity are not fully understood. For example, it is still debated whether heterogeneity arises from different presynaptic exocytosis rates or different success probability of spike triggering at the postsynapse (Grant et al., 2010). One hypothesis to account for ANF heterogeneity has been that total presynaptic $\mathrm{Ca}^{2+}$ conductance might vary across synapses (Sumner et al., 2002; Frank et al., 2009). However, how exactly such a mechanism would operate in the $\mathrm{Ca}^{2+}$ nanodomain control of exocytosis is not clear. In this work, we show how differences in $\mathrm{Ca}^{2+}$ channel density and total number might account for the ANF heterogeneity. We also show that a " $\mathrm{Ca}^{2+}$ microdomain approximation" is an appropriate simplification when investigating ANF heterogeneity. Finally, we find a link between the phenomenological description of ANF rate-level functions (Sachs and Abbas, 1974; Heil et al., 2011) and exocytosis-voltage curves. In particular, we find that the power parameter in rate-level functions is not related to $\mathrm{Ca}^{2+}$ sensor cooperativity, but rather to the sigmoidal character of the relationship between exocytosis and membrane potential, which would arise even for a non-cooperative $\mathrm{Ca}^{2+}$ sensor for exocytosis. 
Despite the auditory system's capability to detect time differences in the order of tens of $\mu$ s (Carr, 1993), at low sound intensities, sound detection can take up to $100 \mathrm{~ms}$. The presence of these very different time scales in the auditory system has been named as the resolution-integration paradox. Long latencies have been classically attributed to the fact that the auditory system integrates sound energy and sound perception occurs when this integral reaches a detection threshold (Hughes, 1946; Heil and Neubauer, 2003). A solution to this integration-resolution paradox was that long latencies result from low spiking probability in the ANF (Krishna, 2002; 2006). In this work we propose that in the $\mathrm{Ca}^{2+}$ nanodomain control of exocytosis, it is the rare $\mathrm{Ca}^{2+}$ channel openings that would account for long latencies. In addition, we show that this result can be reformulated in terms of a temporal integral of sound pressure.

The auditory system analyzes sound frequency not only using the Fourier transform performed by the cochlea (place code), but also by reading out precise spike patterns in individual ANF, which convey information about the precise phase of the sound (temporal code) (Moller, 2006). ANF phase locks to tones of up to $2 \mathrm{kHz}$ in frequency (Moser et al., 2006). This might seem difficult to achieve in the $\mathrm{Ca}^{2+}$ nanodomain control of exocytosis, where locking should be produced by only a few stochastic ion channels. Here, we show that a few ion channels can be as effective in locking release to the stimulus, as would 40 ion channels. This can mainly be attributed to the fact that single ion channel stochasticity is compensated by large $\mathrm{Ca}^{2+}$ concentration variations resulting from the channel openings. This is a signature of stochastic resonance, where noise (the individual channel openings) increases the quality of signal transmission. 


\subsection{Results}

\subsection{1 $\mathrm{Ca}^{2+}$ sensor sensitivity and $\mathrm{Ca}^{2+}$ concentration at the IHC ribbon synapse}

To better understand the response characteristics of the $\mathrm{Ca}^{2+}$ sensor of the IHC (Beutner et al., 2001) in terms of its sensitivity and dynamic range, we calculated the vesicle fusion rate as a function of $\mathrm{Ca}^{2+}$ concentration. We added to the secretion scheme a replenishment step that connected the released state of the scheme to the first state. First, we set the replenishment rate to a very high value $(10 \mathrm{GHz})$ to investigate the sensitivity of exocytosis alone (Fig. 6.1a). The exocytosis rate increased mostly linearly in this range of $\mathrm{Ca}^{2+}$ concentrations. These rates can be seen as release rates per vesicle (or per release site) when the vesicle pool is fully replenished. Then, to investigate the sensitivity of the sensor in the presence of a physiological replenishment, we set the replenishment rate per release site to $40 \mathrm{~Hz}$. For ten release sites it yields a maximum release rate of $400 \mathrm{~Hz}$, which is consistent with the maximum steady state rates at the IHC ribbon synapses (Pangrsic et al., 2010). As a result, the dynamic range of release was for $\mathrm{Ca}^{2+}$ concentrations between 5 and $25 \mu \mathrm{M}$ (Fig. 6.1a). Here, the maximum rate of exocytosis was determined by the replenishment rate, and it fixed the upper bound of the sensitivity range for steady state release.

To understand the physiological relevance of these $\mathrm{Ca}^{2+}$ concentrations, we first calculated the steady state concentration resulting from the opening of a single open $\mathrm{Ca}^{2+}$ channel (Fig. 6.1b). Channel current was set to $0.15 \mathrm{pA}$ and buffering conditions were $0.5 \mathrm{mM}$ EGTA and $0.5 \mathrm{mM} \mathrm{BAPTA}$, an approximation of physiological conditions. Then, we calculated the $\mathrm{Ca}^{2+}$ concentration resulting from a homogeneous $\mathrm{Ca}^{2+}$ influx through a $100 \mathrm{~nm}$ wide by $300 \mathrm{~nm}$ long rectangle (Meyer et al., 2009) (Fig. 6.1c). In this case, we set the total current equivalent to 10 open channels. The $\mathrm{Ca}^{2+}$ concentration did not exceed $40 \mu \mathrm{M}$ in the whole region. Such a homogeneous $\mathrm{Ca}^{2+}$ influx can be seen as a " $\mathrm{Ca}^{2+}$ microdomain approximation" of the real $\mathrm{Ca}^{2+}$ signal (Fig. 6.1d). As $\mathrm{Ca}^{2+}$ concentrations were calculated in the linear approximation, concentrations increased linearly with channel number. We then calculated $\mathrm{Ca}^{2+}$ concentrations in the microdomain approximation at different distances from the $\mathrm{Ca}^{2+}$ channel cluster border (Fig. 6.1e). We see from this figure that depending on the position of the $\mathrm{Ca}^{2+}$ sensor, the opening of only $10-25$ channels is necessary to produce a maximum release rate in the presence of replenishment. 
a

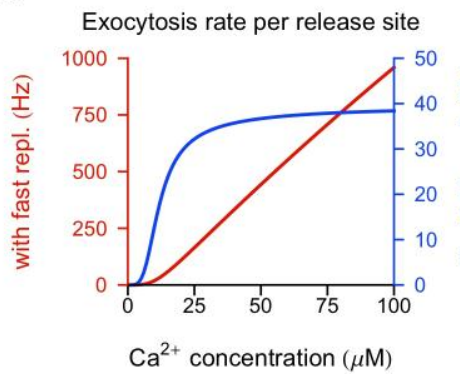

C

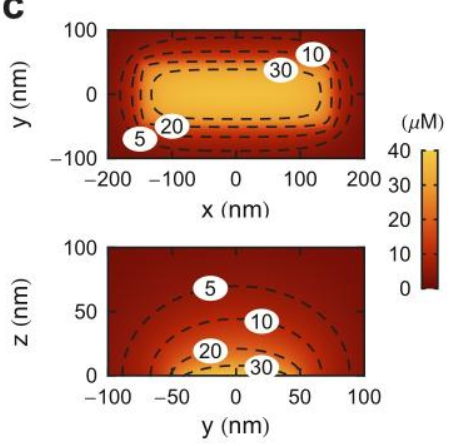

e

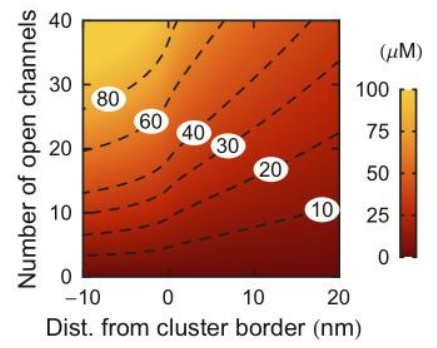

b

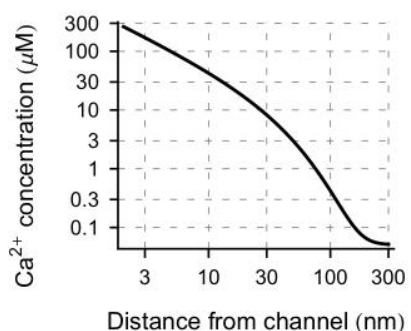

d

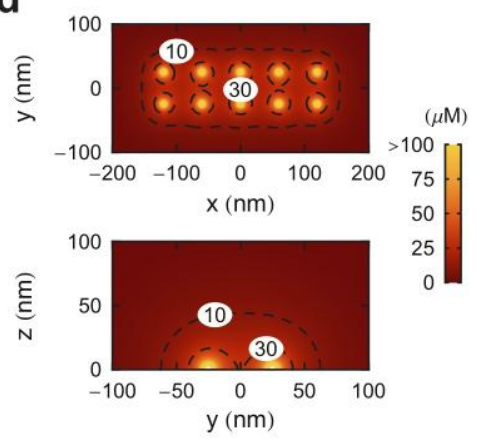

Figure 6.1: $\mathrm{Ca}^{2+}$ sensitivity of exocytosis and $\mathrm{Ca}^{2+}$ concentration at the active zone (a) Steady state release rate per release site as a function of $\mathrm{Ca}^{2+}$ concentration. Red: infinitely fast replenishment of the release site. Blue: replenishment rate of $40 \mathrm{~Hz}$. For steady state release the dynamic range in terms of $\mathrm{Ca}^{2+}$ concentration was from 5 to $25 \mu \mathrm{M}$.

(b) $\mathrm{Ca}^{2+}$ concentration as a function of distance from an open $\mathrm{Ca}^{2+}$ channel with current 0.15 $\mathrm{pA}$, in a log-log scale. Buffering conditions are $0.5 \mathrm{mM}$ EGTA and $0.5 \mathrm{mM}$ BAPTA. The background concentration is $50 \mathrm{nM}$ and is reached at long distances $(>200 \mathrm{~nm})$ from the ion channel.

(c) $\mathrm{Ca}^{2+}$ microdomain approximation : $\mathrm{Ca}^{2+}$ current flowing homogeneously though a rectangle of $300 \mathrm{~nm} \times 100 \mathrm{~nm}$. Total current equivalent to 10 open channels. Top: top view $(x-y$ plane) at $z=1 \mathrm{~nm}$. Bottom: side view ( $y-z$ plane) at $x=0 \mathrm{~nm}$.

(d) Same as (c) but current flowing through 10 regularly distributed channels.

(e) $\mathrm{Ca}^{2+}$ concentration in microdomain approximation as a function of number of open channels and position from the border of the rectangle. $z=0 \mathrm{~nm}, x=0 \mathrm{~nm}$ and varying $y$. The border of the $\mathrm{Ca}^{2+}$ cluster is at $\mathrm{y}=50 \mathrm{~nm}$. 


\subsubsection{Different $\mathrm{Ca}^{2+}$ channel and vesicle positioning could account for auditory nerve fiber functional heterogeneity}

To understand if the differences in $\mathrm{Ca}^{2+}$ channel number per synapse influences synapse sensitivity, we first calculated the steady state release rates per synapse at different membrane potentials for different channel complements per synapse (10-80) in the microdomain approximation. For the channel open probability, we used the mean open probability curve from (Brandt et al., 2005), going from 0 at hyperpolarized voltages to 1 at depolarized voltages (Fig. 6.2a). 10 vesicles were positioned at varying distances from the $\mathrm{Ca}^{2+}$ cluster (Frank et al., 2010). For a given number of channels and distances from the active zone, we found that exocytosis rate versus voltage relationship was sigmoidal, well fit by a Boltzmann curve (Fig. 6.2b):

$$
R(V)=\frac{R_{\text {Max }}}{1+\exp \left(-\left(V-V_{\text {balf }}\right) / V_{\Delta}\right)}
$$

Where $R_{\text {Max }}$ is the maximum exocytosis rate, $V_{\text {balf }}$ is the membrane potential at the mean exocytosis rate and $V_{\Delta}$ is a proxy of the dynamic range. We systematically explored variations in $V_{\text {half }}$ and $V_{\Delta}$ as a function of the number of channels and the distance of vesicles from the active zone (Fig. 6.2c,d). We found that $V_{\text {half }}$ varied in a $30 \mathrm{mV}$ range, which could potentially account for the ANF heterogeneity. Additionally $V_{\Delta}$ remained relatively constant in an extended region of the parameter space.

To understand how heterogeneity could arise in the nanodomain regime, we calculated the exocytosis rate versus voltage curves for different number of channels per vesicle and different vesicle-channel distances (Fig. 6.2e,f). We considered a single characteristic vesicle that sensed the concentration of 1 to 5 channels, positioned at an equal distance from the vesicle. We used a two state Markov model of the ion channel, with Boltzmann factors to express the voltage dependence of the gating rates. The mean open time was $0.2 \mathrm{~ms}$ at $-45 \mathrm{mV}$ (Rodriguez-Contreras and Yamoah, 2001) and changed only from $0.17 \mathrm{~ms}$ to $0.25 \mathrm{~ms}$ from -80 to $0 \mathrm{mV}$. We reported exocytosis rates for 10 vesicles. We found that different number of channels per vesicle or different vesiclechannel distances could account for the heterogeneity found in ANF in the nanodomain description as well. In contrast to the microdomain approximation, $V_{\Delta}$ decreased with increasing distance. This could be explained by the fact that when an ion channel is closely coupled to a vesicle, each opening produce vesicle release, therefore the exocytosis is not negligible already at very low membrane potentials and this resulted in a more extended dynamic range. 
a

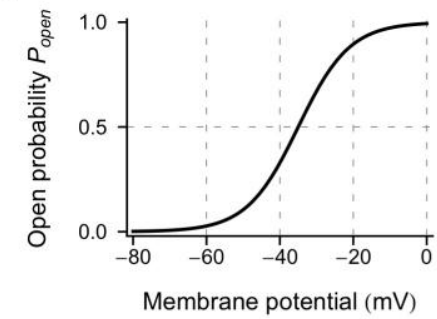

C

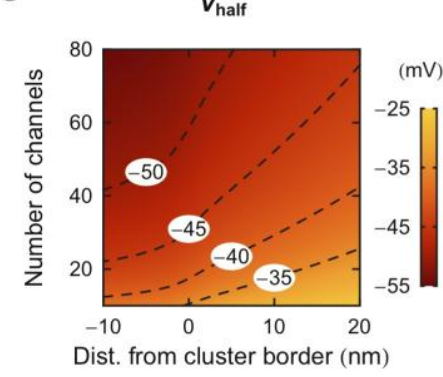

e

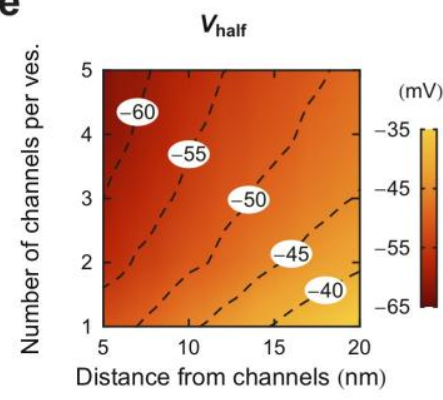

b

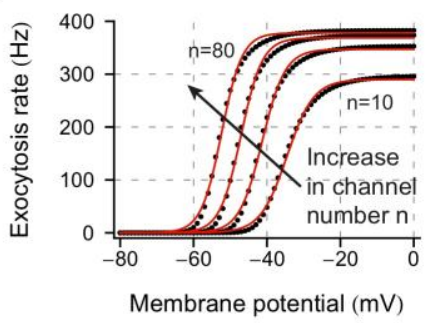

d

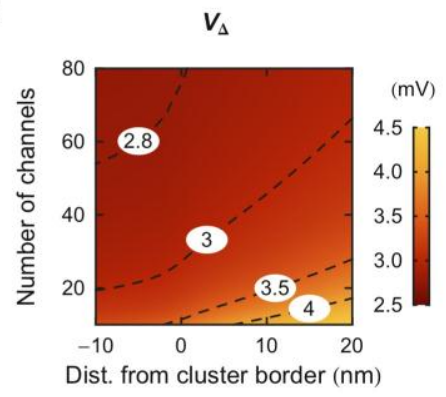

f

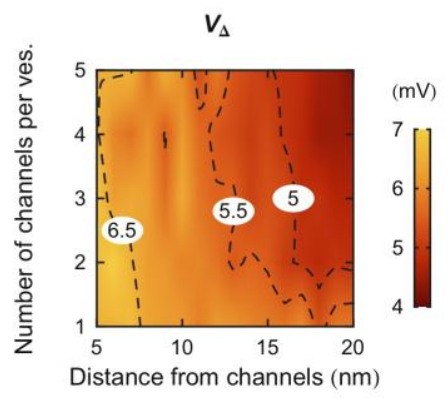

Figure 6.2: auditory nerve fiber heterogeneity could arise from different $\mathrm{Ca}^{2+}$ channel number at the active zone and vesicle-channel positioning

(a) $\mathrm{Ca}^{2+}$ channel open probability as a function of membrane potential.

(b) Steady state exocytosis rates per synapse in the $\mathrm{Ca}^{2+}$ microdomain approximation for different number of $\mathrm{Ca}^{2+}$ channels per synapse: 10, 20, 40 and 80. The exocytosis-voltage curves (black points) are well fit by a Boltzmann function (red curve), equation (6.1). Vesicle were positioned at the border of the $\mathrm{Ca}^{2+}$ cluster, i.e., $y=50 \mathrm{~nm}$.

(c,d) $V_{\text {half }}$ and $V_{\Delta}$ from the Boltzmann fit for different values of channel number and position of the vesicles from the $\mathrm{Ca}^{2+}$ cluster. Negative distance values correspond to a position inside the $\mathrm{Ca}^{2+}$ cluster. Whereas $V_{\text {half }}$ varies extensively in this parameter range, $V_{\Delta}$ is rather constrained.

$(e, f)$ Same as $(c, d)$ but calculated in the nanodomain regime. A vesicle sensed the $\mathrm{Ca}^{2+}$ coming from only 1 to 5 vesicles, all positioned at an equal distance. In this case $V_{\text {half }}$ also varies extensively in this parameter range, $V_{\Delta}$ is rather constrained. $V_{\Delta}$ was larger than in the $\mathrm{Ca}^{2+}$ microdomain approximation and $V_{\text {half }}$ was smaller, because stochastic channel openings elicited release at lower membrane potentials. 
In summary, whereas only 10-25 open $\mathrm{Ca}^{2+}$ channels are necessary to reach the maximum release rate, a higher number of channels enables to reach the maximum release rate at lower voltages. Thus, varying the number of channels at a synapse can modulate the dynamic range of exocytosis in a way that resembled experimental rate-level functions.

If we consider that the potential of an IHC in vivo is around $-55 \mathrm{mV}$ and that it contains 4 synapses with the number of channels as in Fig. 6.2b, then not only will the 4 synapses have a different threshold and sensitivity, but they will also have a different spontaneous exocytosis rate (exocytosis rate in the absence of stimulation). The sigmoidal relationship between exocytosis rate versus voltage implies that the synapses with a high spontaneous rate will be very sensitive so small changes in membrane potential, whereas synapses with a low spontaneous rate will be less sensitive and will require a larger change in membrane potential to have a sensitive change in exocytosis rate. This is consistent with the negative correlation observed experimentally between spontaneous rate and threshold. In conclusion this correlation is a direct signature of the sigmoidal activation curve of ion channels.

\subsubsection{Relation of exocytosis-voltage curves to the rate-level functions}

To better understand the implications of the voltage-exocytosis relationship in terms of the rate-level functions, we transformed the membrane potential into sound pressure level (SPL) and the exocytosis rate into spike rate. When ANFs are stimulated below their best (characteristic) sound frequency, the amplification dynamics is linear (Patuzzi and Sellick, 1983; Heil et al., 2011). Therefore we can assume a "shifted" linear relationship between SPL $=20 \log _{10}\left(\mathrm{P} / \mathrm{P}_{\text {ref }}\right)$ and IHC membrane potential $V$ :

$$
V(P)=\theta \cdot 20 \log _{10}\left(\left(P+P_{0}\right) / P_{r e f}\right)+V_{0}
$$

Where $\theta$ is a linear factor, $P_{0}$ and $V_{0}$ are the "shift factors" for the pressure and the voltage, respectively. $P$ represents the sound pressure envelope (relatively to atmospheric pressure) and $P_{r e f}$ is a reference pressure (usually $20 \mu \mathrm{Pa}$ ). In addition we assumed that each released vesicle from the IHC had a fixed probability to generate a spike in the ANF: we set a linear relationship between the vesicle release rate $R_{V}$ and the spike rate Rs:

$$
R_{S}=\lambda \cdot R_{V}
$$

Using equations (6.1), (6.2) and (6.3) we derived the spike rate as a function of sound pressure: 


$$
R_{S}(P)=\frac{\lambda \cdot R_{\text {Max }} \cdot\left(P+P_{0}\right)^{\beta}}{K^{-1}+\left(P+P_{0}\right)^{\beta}}
$$

where $\beta=20 \theta /\left(V_{\Delta} \log (10)\right)$ and $K^{-1}=\left(P_{\text {ref }}\right)^{\beta} \exp \left(\left(V_{\text {balf }}-V_{0}\right) / V_{\Delta}\right)$.

The equations (6.4) is exactly the same phenomenological relationship obtained by Heil and colleagues (Heil et al., 2011) when fitting the rate-level functions. They found that $K^{-1}$ determined the sensitivity of fibers, whereas $\beta$ was constant across all fibers and has the value of $\beta \approx 3$. From there we can conclude that the ratio of $V_{\Delta}$ and $\theta$ might be similar across all ANF fibers. The power $\beta$ was interpreted as the cooperativity factor of the $\mathrm{Ca}^{2+}$ sensor (Heil et al., 2011). Our results argue that the power arises simply because of the sigmoidal relationship between release rate and voltage, which is not directly imposed by the cooperativity of the sensor. In fact the sigmoidal relationship arises from mainly two effects: (1) the slow increase of exocytosis with voltage is imposed by the activation the ion channels; (2) the saturation is present due to the replenishment rate. Therefore the power law and $\beta \approx 3$ could still be present even if the relationship between $\mathrm{Ca}^{2+}$ concentration and release was linear.

\subsubsection{Resolution-integration paradox in the auditory system}

For an $\mathrm{ANF}$ with a near $0 \mathrm{~Hz}$ spontaneous rate, a small increase in sound pressure level would result in a long latency spike. The latency $L$ of this spike can be effectively expressed as the inverse of the rate (6.4) found previously:

$$
\begin{gathered}
L(P)=1 / R(P)=\frac{K^{-1}\left(P+P_{0}\right)^{-\beta}+1}{\lambda \cdot R_{\text {Max }}}=C_{1}\left(P+P_{0}\right)^{-\beta}+C_{2} \\
=C_{1}\left(\int_{0}^{L} P(t)+P_{0} d t / L\right)^{-\beta}+C_{2} \\
L^{*}(P) \cdot\left(\int_{0}^{L} P(t)+P_{0} d t / L\right)^{\beta}=\text { const. }
\end{gathered}
$$

where $C_{1}$ and $C_{2}$ are constants and $L^{*}=L-C_{2}$. This expression corresponds to the result of Heil and colleagues (Heil and Neubauer, 2003) without the $P_{0}$ factor.

Although the latency $L$ is expressed through an integral of $P(t)$, long latencies do not result from an integration process, but simply from a low vesicle release probability. Again, the "power law" between the integral of sound pressure envelope and latency results from the sigmoidal voltage-exocytosis rate relationship rather than from a $\mathrm{Ca}^{2+}$ sensor cooperativity. Finally, in the $\mathrm{Ca}^{2+}$ nanodomain control of exocytosis, long 
latencies are due to very rare ion channel openings rather than to low $\mathrm{Ca}^{2+}$ concentrations induced by the openings (Meddis, 2006; Heil et al., 2011). The low $\mathrm{Ca}^{2+}$ concentration could indeed be viewed as the temporal mean of the concentration resulting from rare ion channel openings. This simplification would however be meaningful only if the relationship between $\mathrm{Ca}^{2+}$ concentration and release rate was linear, which is not the case in reality (Beutner et al., 2001).

\subsubsection{Phase locking: are 2 channels more precise than 40 ?}

It might seem that just one ion channel controlling vesicle exocytosis is not only unreliable, but also imprecise. This would in particular be noticeable when vesicle release should be phase locked to a sound tone. Here we probed how well vesicle release can be locked to a sinusoidal voltage stimulus in the $\mathrm{Ca}^{2+}$ microdomain and nanodomain regimes. We used a $4 \mathrm{mV}$ peak-to-peak amplitude voltage stimulus at $500 \mathrm{~Hz}$ and calculated the mean exocytosis rate per cycle (Fig. 6.3a). For different scenarios, we chose as mean voltage the most sensitive voltage $\left(V_{\text {balf }}\right)$ from the micro and nanodomain regimes (Fig. 6.2c-f). The quality of the phase locking was assessed by the synchronization index (SI) (Johnson, 1980):

$$
\mathrm{SI}=\left\|\int d t r(t) \exp (i 2 \pi f t) / \int d t r(t)\right\|
$$

Depending on the chosen nanodomain configuration in terms of number of ion channels per vesicle and distance from vesicle to channel, the locking was worse, similar, or even better than in the microdomain approximation (Fig. 6.3b). The fact that the nanodomain can perform so well can be explained by the following: even though the $\mathrm{Ca}^{2+}$ concentration oscillations are less precise when they are govern by stochastic channels than in the microdomain regime, they cover a much larger range of $\mathrm{Ca}^{2+}$ concentrations. The precise determinants of phase locking quality and the transition between micro and nanodomain still remain to be investigated. 
a
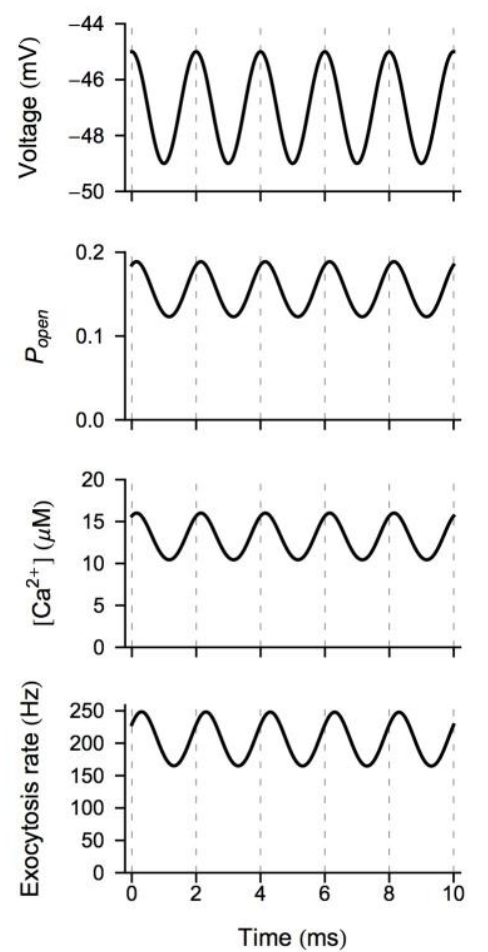

b

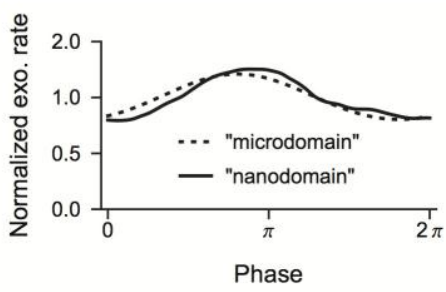

Figure 6.3: phase locking in micro and nanodomain

(a) A voltage sinusoid of $500 \mathrm{~Hz}$ was applied (top). This produced oscillations in the channel open probability (second from the top) with a small phase shift. From there, the time course of the $\mathrm{Ca}^{2+}$ concentration was calculated (in the microdomain approximation, third from the top) and exocytosis rates were derived (bottom). The maximum $\mathrm{Ca}^{2+}$ current through the channel cluster rectangle was homogeneous and corresponded to 40 channels. Vesicles were positioned on the border of the $\mathrm{Ca}^{2+}$ channel cluster.

(b) One phase of the exocytosis rate for the micro and nanodomain regimes. In this example phase locking was slightly better in the nanodomain regime than in the microdomain regime with synchronization indexes of 0.11 and 0.10 , respectively. For the nanodomain were used 2 channels at $5 \mathrm{~nm}$ distance from the vesicle. Voltage oscillations were around $-56 \mathrm{mV}$ in the nanodomain regime. The rate was normalized so that the mean rate was 1 . 


\subsection{Discussion}

We investigated the consequences of $\mathrm{Ca}^{2+}$ channel nanodomain to vesicle release coupling on synapse functioning and on system level behavior.

We showed that changes in $\mathrm{Ca}^{2+}$ channel number could account for ANF heterogeneity. We demonstrated that the power coefficient describing ANF rate level functions is mainly set by the sigmoidal relationship between steady-state release exocytosis and membrane potential. Having many channels might not be useful to reach a high $\mathrm{Ca}^{2+}$ concentration or even higher release rates, but rather to reach the maximum release rate at lower voltages, and thus sound pressure levels. In this way, different ranges of SPL can be covered by different synapses and ANFs. Finally, although it has been often questioned what is the computational advantage of a negative correlation between ANF spontaneous rate and ANF threshold (Liberman, 1982), we argued here that this correlation might be simply unavoidable.

We showed that the long latencies are directly related to the low release rate of low spontaneous rate $\mathrm{ANF}$. Also we favor the interpretation that it is rare $\mathrm{Ca}^{2+}$ openings that produce long delays rather than a steady state low $\mathrm{Ca}^{2+}$ concentration.

Finally, we showed that a few $\mathrm{Ca}^{2+}$ channel could produce a phase locking equivalent to that produced by an order of magnitude more ion channel. This could be explained by larger variations of $\mathrm{Ca}^{2+}$ concentration that a smaller number of channels produce. This property might be related to stochastic resonance, when noise (here channel openings) can enable a higher sensitivity of the system. But it still remains to be investigated which are the critical factors of channel-vesicle coupling that produce different quality of phase locking. In particular it needs to be determined where lies the optimum in the transition between nano- and microdomain regimes. 


\subsection{Methods}

Calculations and simulations were performed in Mathematica (Wolfram Research).

\subsection{1 $\mathrm{Ca}^{2+}$ channel model}

For simplicity, we chose a two state $\mathrm{Ca}^{2+}$ channel model:

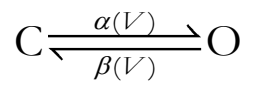

where $\mathrm{O}$ and $\mathrm{C}$ stand for the open and closed states, respectively. $\alpha(V)$ and $\beta(V)$ are the voltage dependent transition rates:

$$
\alpha(V)=A_{1} \exp \left(A_{2} V\right), \beta(V)=B_{1} \exp \left(B_{2} V\right)
$$

were $A_{i}$ and $B_{i}$ are constants. These constants were chose so that the resulting mean open probability $P_{\text {open: }}$ :

$$
P_{\text {open }}(V)=\alpha(V) /(\alpha(V)+\beta(V))
$$

was similar to (Brandt et al., 2005), going from 0 at hyperpolarized membrane potentials to 1 at depolarized potentials. In addition we chose $\beta(V)$ to vary slowly with voltage and giving a mean channel open time around $0.2 \mathrm{~ms}$ (Rodriguez-Contreras and Yamoah, 2001). The remaining constants were determined by the previously stated constrains and were:

$\mathrm{A}_{1}=594 \mathrm{~ms}^{-1} ; \mathrm{A}_{2}=0.138 \mathrm{mV}^{-1} ; \mathrm{B}_{1}=4 \mathrm{~ms}^{-1} ; \mathrm{B}_{2}=0.005 \mathrm{mV}^{-1}$.

We did not model the gating modes of the channel (Rodriguez-Contreras and Yamoah, 2001; Zampini et al., 2010), considering it equivalent to a smaller number of "effective" channels at the active zone.

The evolution of the open state is given by:

$$
\mathrm{O}^{\prime}(t)=-\beta(V(t)) \cdot \mathrm{O}(t)+\alpha(V(t)) \cdot(1-\mathrm{O}(t))
$$

and was calculated either in mean field (in the microdomain regime), or stochastically (in the nanodomain regime) using the "first reaction" method (Gillespie, 1976).

\subsubsection{Buffer environment and $\mathrm{Ca}^{2+}$ current}

We chose $0.5 \mathrm{mM}$ EGTA and $0.5 \mathrm{mM}$ BAPTA as $\mathrm{Ca}^{2+}$ buffers with binding and unbinding rates from (Naraghi and Neher, 1997). These concentrations of buffer approximate physiological buffering (Hackney et al., 2005) (Tina Pangrsic, personal communication). Single channel current was $0.15 \mathrm{pA}$ and was set to not vary with membrane potential, considering the small voltage range of interest $(-60 \mathrm{mV}$ to $-30 \mathrm{mV})$. This is the current expected at physiological conditions at $-30 \mathrm{mV}$ (assuming a 
conductance of $2.1 \mathrm{pS}$ and a reversal potential of $42 \mathrm{mV}$ as in (Rodriguez-Contreras et al., 2002) ). The background $\mathrm{Ca}^{2+}$ concentration was fixed to $50 \mathrm{nM}$.

\subsubsection{Microdomain regime of exocytosis}

To calculate the release rate as a function of voltage, we considered a rectangle of $100 \mathrm{x}$ $300 \mathrm{~nm}$ through which $\mathrm{Ca}^{2+}$ current could flow (Meyer et al., 2009). We calculated the resulting $\mathrm{Ca}^{2+}$ concentration for different number of total channels using the linear solution of (Naraghi and Neher, 1997). The $\mathrm{Ca}^{2+}$ concentration at a position (x,y,z) was given by:

$$
\begin{aligned}
& {[C a](x, y, z, t)=} \\
& {[C a]_{\infty}+\mathrm{O}(t) \cdot \frac{N_{c b}}{100 \cdot 300} \int_{-50}^{50} d x^{\prime} \int_{-150}^{150} d y^{\prime} \delta[C a]\left(\sqrt{\left(x-x^{\prime}\right)^{2}+\left(y-y^{\prime}\right)^{22}+z^{2}}\right)}
\end{aligned}
$$

where $\delta[\mathrm{Ca}](r)$ is the $\mathrm{Ca}^{2+}$ concentration at a distance $r$ resulting from a single open ion channel, $N_{c b}$ is the number of channels, $[\mathrm{Ca}]_{\infty}$ is the background $\mathrm{Ca}^{2+}$ concentration and $\mathrm{O}(t)$ is the mean channel open probability at time $t$. Thus the $\mathrm{Ca}^{2+}$ concentration instantaneously followed changes of the mean channel open probability $\mathrm{O}(t)$.

\subsubsection{Nanodomain regime of exocytosis}

In the nanodomain regime, the individual ion channel gating matters for vesicle exocytosis. We placed 1 to $5 \mathrm{Ca}^{2+}$ channels at distance $d$ from the $\mathrm{Ca}^{2+}$ sensor of a vesicle. We calculated the stochastic gating of the ion channels. The opening of the ion channel produced an immediate increase of the $\mathrm{Ca}^{2+}$ concentration, equal to the steady state concentration. Opening of additional ion channels simply resulted in the sum of their respective contributions, as assumed by the linear approximation (Naraghi and Neher, 1997). The closure of a $\mathrm{Ca}^{2+}$ channel resulted in an instantaneous decrease of the concentration to the steady state concentration.

\subsubsection{Vesicle cycle}

Exocytosis was modeled using the (Beutner et al., 2001) scheme using the $\mathrm{Ca}^{2+}$ concentration obtained either from the micro- or the nanodomain regime. The last $\mathrm{Ca}^{2+}$ independent release rate was set to $10 \mathrm{~ms}^{-1}$ as in (Millar et al., 2005). Replenishment per release site was $40 \mathrm{~Hz}$ per vesicle and was $\mathrm{Ca}^{2+}$-independent. 


\section{Discussion}

In this work we studied how several aspects of the synaptic transmission from the inner hair cell onto the auditory nerve fibers contribute to sound encoding. In chapter 3 we investigated how fast vesicle replenishment might be supported by the presence of the synaptic ribbon and proposed that it could function as a "Brownian conveyor trap". In chapter 6 we examined how $\mathrm{Ca}^{2+}$ nanodomain control of exocytosis might contribute to precise sound encoding, and how variations in $\mathrm{Ca}^{2+}$ channel number per synapse could enable encoding of different sound intensity ranges. In chapter 4 and 5 we explored the candidate mechanisms underlying large and variable EPSC shapes triggered by neurotransmitter release from the inner hair cell ribbon synapse. Our results suggest that large EPSCs might result from the exocytosis of just one vesicle, rather than many vesicles as classically assumed. Finally in chapter 2, we investigated the postsynaptic specialization of spiral ganglion neurons (SGNs) that enables them to reliably encode the sound signal. We found several distinguishing features: SGNs were phasic, had a short integration time constants, a low spiking threshold and a sharp spike initiation mechanism. These properties equip SGNs to precisely encode the timing of almost every neurotransmitter release coming from the inner hair cell.

This work as a whole gives the intriguing picture that the brain might detect very minute changes happening at the protein level. In fact, the conformational change of a single $\mathrm{Ca}^{2+}$ channel resulting in its opening, might elicit the release of a vesicle, which might trigger an action potential in the auditory nerve fiber.

In this last part of the work, we will discuss different aspects of these encoding capabilities in a more general context.

\subsection{Fast synaptic transmission and role of the synaptic ribbon}

In this work, we suggested that the presence of the ribbon might contribute to high vesicle replenishment rates in ribbon synapses (chapter 3). An important question in the synaptic field is which step in the vesicle cycle limits even higher exocytosis rates (Neher, 2010). Although vesicle resupply might be a limiting factor (Rizzoli and Betz, 2005), it seems that each release site at the active zone bears even higher demands during sustained exocytosis. In fact, after vesicle exocytosis, the release site must be ready to dock and prime a new coming vesicle. In the case of the IHC ribbon synapses, which is thought to contain around 10 release sites (Frank et al., 2010) and can sustain exocytosis 
rates of up to $600 \mathrm{~Hz}$ (Pangrsic et al., 2010), a release-site would need to be prepared in less than $16 \mathrm{~ms}$. In this short time new fusion competent proteins need to be made available and positioned at the appropriate spot. Thus, release site availability might be a rate-limiting step of the vesicle cycle, and several arguments argue in this direction for the calyx of Held (Neher, 2010). But if that is also the rate-liming step for the IHC ribbon synapse is not clear.

From the synapse point of view, each step of the vesicle cycle requires metabolic energy: preparation of the release site, exocytosis, endocytosis, formation of new vesicles and their transportation to the plasma membrane. To make the neurotransmission cycle faster, each of these steps might need to be enhanced, which would require additional energy. When a synapse is releasing at its maximum rates, are all the steps at their maximum output, or only the rate-limiting one? Since synaptic transmission is never "infinitely fast", there will always be a rate-limiting step. However, it is not clear whether this limiting step was chosen for energy consumption or computation purposes, or was imposed by fundamental biophysical principles. Knowing that IHC ribbon synapses are capable to exocytose at $600 \mathrm{~Hz}$ at steady state, one is lead to argue that there shouldn't be any biophysical limitation to exocytose up to such a high rate. Thus one should conclude that any slower synapse is so by "choice" or by "design", to effectively perform what it is "required" to do, and not more.

What are the specializations that enable the IHC ribbon synapses to be fast and which role does the ribbon play in that respect? Bassoon mutations, which result in an unanchored ribbon, display exocytosis defects (Dick et al., 2003; Khimich et al., 2005; Buran et al., 2010; Frank et al., 2010), and would argue for an important role of the ribbon in the vesicle cycle. Some ribbon-less synapses, however, are almost as fast as ribbon synapses: for example the cerebellar mossy fibers (Saviane and Silver, 2006; Rancz et al., 2007). In contrast, some synapses with a ribbon are slower: for example the rod bipolar cell (Singer et al., 2004). It has been suggested that the ribbon might even prevent vesicle arrival to the active zone, so that the transient and sustained component of release are well distinguishable (Jackman et al., 2009). These considerations raise the questions whether the primary role of the ribbon is really to accelerate vesicle arrival to the release sites, or if the ribbon is rather important for example for computational purposes, i.e., a pool of vesicles with specific dynamics. For example, Jarsky et al., 2011; Oesch and Diamond, 2011) have shown that rod bipolar cells are able to compute light temporal contrast mainly thanks to the vesicle pool dynamics. It should of course not be excluded that the role of the ribbon might be different in different synapses. In conclusion, the 
role of the ribbon still remains an open question and it seems that its presence it neither a sufficient nor a necessary condition for a synapse to be fast.

One possible approach to address the role of the ribbons is to unravel its exact molecular composition and its implications in vesicle docking, priming and exocytosis. (Schmitz, 2009; Schwarz et al., 2011).

\section{2 $\mathrm{Ca}^{2+}$ nanodomain and microdomain control of exocytosis}

At a synapse, the average distance between $\mathrm{Ca}^{2+}$ channels and vesicles has a significant functional relevance (Chad and Eckert, 1984; Yamada and Zucker, 1992; Neher, 1998b; Augustine et al., 2003; Matveev et al., 2011). This distance establishes how intimately the release of a vesicle is related to the opening of single $\mathrm{Ca}^{2+}$ channels. In the nanodomain regime, individual ion channel openings determine vesicle exocytosis timing. In the microdomain regime, it is rather the global $\mathrm{Ca}^{2+}$ concentration, built by an ensemble of $\mathrm{Ca}^{2+}$ channels, which is responsible for the vesicle release rates. Also, in the nanodomain regime, delays between $\mathrm{Ca}^{2+}$ influx and exocytosis can be smaller than in the microdomain regime: firstly, because a smaller time is needed for $\mathrm{Ca}^{2+}$ to diffuse from the channel to the vesicle and secondly, because $\mathrm{Ca}^{2+}$ concentrations can be higher near the channel mouth and thus produce faster exocytosis. The latter, however, depends on the $\mathrm{Ca}^{2+}$ sensor sensitivity.

$\mathrm{Ca}^{2+}$ nanodomain control of exocytosis has been found at ribbon synapses (Brandt et al., 2005; Goutman and Glowatzki, 2007; Jarsky et al., 2010) as well as at the squid giant synapse (Adler et al., 1991), at a fast GABAergic cortical synapse (Bucurenciu et al., 2008; 2010) and at the mature calyx of Held (Fedchyshyn and Wang, 2005). But computational implications of $\mathrm{Ca}^{2+}$ nanodomain control of exocytosis might be different for a spiking and a graded potential cell. In the case of a spiking cell, the timing of $\mathrm{Ca}^{2+}$ influx is roughly determined by the action potential shape and the time course of $\mathrm{Ca}^{2+}$ channel activation and deactivation (Borst and Sakmann, 1999; Bean, 2007). On the other hand, for a graded potential neuron, $\mathrm{Ca}^{2+}$ channels are continuously and stochastically gating between the open and the closed states. This stochastic gating might lead to a noisy $\mathrm{Ca}^{2+}$ influx and an imprecise control of exocytosis in a graded potential cell. But it could also enable stochastic resonance and improve synapse sensitivity (chapter 6). Interestingly, if $\mathrm{Ca}^{2+}$ channel kinetics are very fast, the $\mathrm{Ca}^{2+}$ concentration time course felt by a vesicle near an ion channel could be very similar to the $\mathrm{Ca}^{2+}$ concentration time course produced by a multitude of distant $\mathrm{Ca}^{2+}$ channels. This results from the temporal low-pass filtering of a rapidly changing $\mathrm{Ca}^{2+}$ concentration. Thus the exact $\mathrm{Ca}^{2+}$ channel 
kinetics might be crucial to understand the advantages of each regime of exocytosis control as well as to describe the transition between them.

\subsection{Synapse sensitivity modulation with the number of $\mathrm{Ca}^{2+}$ channels}

In a spiking neuron synapses, variations in the number of $\mathrm{Ca}^{2+}$ channels can change the mean $\mathrm{Ca}^{2+}$ concentration during an action potential and strongly affect the vesicle release probability (Schneggenburger and Neher, 2005). Thus, varying the $\mathrm{Ca}^{2+}$ channel number could modulate synaptic strength. For the IHC ribbon synapses, we found that changing the $\mathrm{Ca}^{2+}$ channel number could change the synapse sensitivity (chapter 6 ). The notions of synaptic strength and sensitivity could be considered equivalent: the former applies in the context of action potential stimulations, whereas the latter in the context of graded potential stimulations.

The modulation mechanism we proposed is certainly not the only mechanism contributing to synapse heterogeneity in IHC ribbon synapses. For examples differences in ribbon sizes have been reported across synapses (Merchan-Perez and Liberman, 1996) and they seem to be correlated with the ANF spontaneous rate: the larger the ribbon, the smaller the spontaneous rate. Also variations in the voltage activation curve of the $\mathrm{Ca}^{2+}$ channel might underlie synaptic heterogeneity (Frank et al., 2009). Finally, additional modulation could arise from the efferent innervation of the ANFs (Liberman, 1980; Groff and Liberman, 2003).

One very difficult experiment that would radically advance our understanding of synaptic heterogeneity in IHC ribbon synapses would be to combine presynaptic $\mathrm{Ca}^{2+}$ imaging with postsynaptic patch-clamp, in order to correlate the presynaptic $\mathrm{Ca}^{2+}$ signal amplitude with responses in the afference.

\subsection{Vesicle fusion pore}

In this work (chapter 4 and 5), we proposed that the origin of large EPSCs triggered by neurotransmitter release from the IHC might be uniquantal - arising from the release of a single vesicle. Also we proposed that the multiphasic EPSCs might arise from flickering vesicle fusion pores. In addition, this interpretation might also apply to other ribbon synapses. Large EPSCs might be uniquantal, whereas smaller EPSCs might be the result of subquantal release due to a premature vesicle fusion pore closure. The main difference between IHC ribbon synapses and other ribbon synapses would be the following: in IHC, a vesicle in which a short fusion pore opening occurs once, will eventually undergo 
full fusion and release all of its neurotransmitter content, whereas in other ribbon synapses, the pore can effectively reclose again after a short opening.

But other interpretations need still to be considered. For example that large EPSCs in other ribbon synapses are uniquantal and that so are also the small EPSCs, but resulting from the exocytosis of the vesicles at other locations. However this might lead to a different EPSC rise time. Finally, although rather unlikely, the MQR interpretation should not be dismissed.

If small EPSCs are indeed due to short pore openings, it would be an extreme example of kiss-and-run exocytosis, where the fusion pore usually remains open for durations in the second or hundreds of milliseconds range (He et al., 2006; Segovia et al., 2010). The exact mechanisms by which a fusion pore is formed and regulated during the exocytosis of a small synaptic vesicles are not entirely understood (Jahn et al., 2003; Jackson and Chapman, 2008; Sørensen, 2009; Soekmadji and Thorn, 2010). One of the hypotheses is that prior to pore formation there is a hemifusion state, where the vesicle membrane inner leaflet is in contact with the plasma membrane outer leaflet. Then, the content of the vesicle is only separated by a lipid bilayer from the synaptic cleft. It could be imaginable that instabilities of the hemifusion state lead to very short pore openings that would permit neurotransmitter escape into the synaptic cleft. Also the molecular fusion machinery might be responsible in controlling or regulating the pore instabilities.

To further test the vesicle pore flickering hypothesis, one option would be to perform on-cell capacitance measurements (He et al., 2006), however it is not clear whether the time resolution of this method would suffice to detect such short events.

Although the molecular key players of exocytosis at cortical synapses are more and more elucidated (Haucke et al., 2011; Walter et al., 2011), the fusion machinery at the IHC ribbon synapse seem to be different in several aspects. First it seems to be devoid of complexins (Strenzke et al., 2009). Mature animals lack synaptotagmins I and II and VII, but these proteins are transiently expressed during maturation (Safieddine and Wenthold, 1999; Beurg et al., 2010). Also the IHC seems to lack conventional SNAREs (Nouvian et al., 2011). Finally otoferlin seems to play an important role in the vesicle cycle (Roux et al., 2006; Pangrsic et al., 2010). These deviations from the conventional release machinery might have important implications on the exocytosis process and might be linked to the observed pore flickering. 


\subsection{Class III excitability}

One of the particularity of many neurons in the auditory pathway is their class III excitability: they fire only one action potential in response to a constant injected current (Oertel, 1983; Rothman et al., 1993; Brew and Forsythe, 1995; Rathouz and Trussell, 1998; Rothman and Manis, 2003; Bahmer and Langner, 2009). This is in contrast to most firing behaviors in the cortex, which are more often class I or class II and which can have a large diversity of response properties, including bursting, irregular spiking, adapting, chattering, regular spiking or accommodating (Markram et al., 2004; Izhikevich, 2007). In our work (chapter 2), we have shown that the first neuron in the auditory pathway is class III, as the neurons further in the auditory pathway. It spikes only in response to fast changes in membrane potential. And it doesn't fire twice even for large and long EPSCs. We conclude that such a design enables the neuron to lock as much as possible to the arrival of neurotransmitter in the synaptic cleft and thus reliably transmit the neurotransmitter release timing. In conclusion, such a simple spiking behavior, which works like a high-pass filter, might be the most suitable to conserve precise timing information. 


\section{References}

Abbott LF (1999) Lapicque's introduction of the integrate-and-fire model neuron (1907). Brain Res. Bull. 50:303-304

Adamson CL, Reid MA, Mo Z-L, Bowne-English J, Davis RL (2002) Firing features and potassium channel content of murine spiral ganglion neurons vary with cochlear location. J Comp Neurol 447:331-350

Adler EM, Augustine GJ, Duffy SN, Charlton MP (1991) Alien intracellular calcium chelators attenuate neurotransmitter release at the squid giant synapse. J Neurosci 11:1496-1507

Allen MP, Tildesley DJ (1989) Computer Simulation of Liquids. Oxford University Press, USA.

Andor-Ardó D, Hudspeth AJ, Magnasco MO, Piro O (2010) Modeling the resonant release of synaptic transmitter by hair cells as an example of biological oscillators with cooperative steps. Proc Natl Acad Sci USA 107:2019-2024

Ashmore J (2008) Cochlear outer hair cell motility. Physiol Rev 88:173-210

Augustine GJ, Santamaria F, Tanaka K (2003) Local calcium signaling in neurons. Neuron 40:331-346

Avissar M, Furman AC, Saunders JC, Parsons TD (2007) Adaptation reduces spike-count reliability, but not spike-timing precision, of auditory nerve responses. J Neurosci 27:6461-6472

Awizio A-K, Onofri F, Benfenati F, Bonaccurso E (2007) Influence of synapsin I on synaptic vesicles: an analysis by force-volume mode of the atomic force microscope and dynamic light scattering. Biophys J 93:1051-1060

Bahmer A, Langner G (2009) A simulation of chopper neurons in the cochlear nucleus with wideband input from onset neurons. Biol Cybern 100:21-33

Bartol TM, Land BR, Salpeter EE, Salpeter MM (1991) Monte Carlo simulation of miniature endplate current generation in the vertebrate neuromuscular junction. Biophys J 59:1290-1307

Bean BP (2007) The action potential in mammalian central neurons. Nat Rev Neurosci $8: 451-465$

Beurg M, Michalski N, Safieddine S, Bouleau Y, Schneggenburger R, Chapman ER, Petit C, Dulon D (2010) Control of exocytosis by synaptotagmins and otoferlin in auditory hair cells. J Neurosci 30:13281-13290

Beutner D, Voets T, Neher E, Moser T (2001) Calcium dependence of exocytosis and endocytosis at the cochlear inner hair cell afferent synapse. Neuron 29:681-690

Borst JG, Sakmann B (1996) Calcium influx and transmitter release in a fast CNS synapse. Nature 383:431-434 
Borst JG, Sakmann B (1999) Effect of changes in action potential shape on calcium currents and transmitter release in a calyx-type synapse of the rat auditory brainstem. Philos. Trans. R. Soc. Lond., B, Biol. Sci. 354:347-355

Brandt A, Striessnig J, Moser T (2003) CaV1.3 channels are essential for development and presynaptic activity of cochlear inner hair cells. J Neurosci 23:10832-10840

Brandt A, Khimich D, Moser T (2005) Few CaV1.3 channels regulate the exocytosis of a synaptic vesicle at the hair cell ribbon synapse. J Neurosci 25:11577-11585

Brette R, Gerstner W (2005) Adaptive exponential integrate-and-fire model as an effective description of neuronal activity. J Neurophysiol 94:3637-3642

Brew HM, Forsythe ID (1995) Two voltage-dependent $\mathrm{K}+$ conductances with complementary functions in postsynaptic integration at a central auditory synapse. $\mathrm{J}$ Neurosci 15:8011-8022

Brunel N, van Rossum MCW (2007) Lapicque's 1907 paper: from frogs to integrate-andfire. Biol Cybern 97:337-339

Bucurenciu I, Kulik A, Schwaller B, Frotscher M, Jonas P (2008) Nanodomain coupling between $\mathrm{Ca} 2+$ channels and $\mathrm{Ca} 2+$ sensors promotes fast and efficient transmitter release at a cortical GABAergic synapse. Neuron 57:536-545

Bucurenciu I, Bischofberger J, Jonas P (2010) A small number of open Ca2+ channels trigger transmitter release at a central GABAergic synapse. Nat Neurosci 13:19-21

Buran BN, Strenzke N, Neef A, Gundelfinger ED, Moser T, Liberman MC (2010) Onset Coding Is Degraded in Auditory Nerve Fibers from Mutant Mice Lacking Synaptic Ribbons. J Neurosci 30:7587-7597

Cao X-J, Oertel D (2010) Auditory nerve fibers excite targets through synapses that vary in convergence, strength, and short-term plasticity. J Neurophysiol 104:2308-2320

Carr CE (1993) Processing of temporal information in the brain. Annu. Rev. Neurosci. 16:223-243

Chad JE, Eckert R (1984) Calcium domains associated with individual channels can account for anomalous voltage relations of CA-dependent responses. Biophys J 45:993-999

Chanturiya A, Chernomordik LV, Zimmerberg J (1997) Flickering fusion pores comparable with initial exocytotic pores occur in protein-free phospholipid bilayers. Proc Natl Acad Sci USA 94:14423-14428

Chen C (1997) Hyperpolarization-activated current (Ih) in primary auditory neurons. Hear Res 110:179-190

Cichocki B, Hinsen K (1990) Dynamic computer simulation of concentrated hard sphere suspensions. I. Simulation technique and mean square displacement data. Physica A 166:473-491

Dallos P (1985) Response characteristics of mammalian cochlear hair cells. J Neurosci 5:1591-1608 
Dayan P, Abbott LF (2004) Theoretical Neuroscience -Computational And Mathematical Modeling Of Neural Systems. MIT Press:432

del Castillo J, Katz B (1954) Quantal components of the end-plate potential. J Physiol (Lond) 124:560-573

Dick O, Tom Dieck S, Altrock WD, Ammermüller J, Weiler R, Garner CC, Gundelfinger ED, Brandstätter JH (2003) The presynaptic active zone protein bassoon is essential for photoreceptor ribbon synapse formation in the retina. Neuron 37:775786

Edmonds BW, Gregory FD, Schweizer FE (2004) Evidence that fast exocytosis can be predominantly mediated by vesicles not docked at active zones in frog saccular hair cells. J Physiol (Lond) 560:439-450

Evans EF (1978) Place and time coding of frequency in the peripheral auditory system: some physiological pros and cons. Audiology 17:369-420

Fatt P, Katz B (1951) An analysis of the end-plate potential recorded with an intracellular electrode. J Physiol (Lond) 115:320-370

Fedchyshyn MJ, Wang L-Y (2005) Developmental transformation of the release modality at the calyx of Held synapse. J Neurosci 25:4131-4140

Fettiplace R, Hackney CM (2006) The sensory and motor roles of auditory hair cells. Nat Rev Neurosci 7:19-29

Fleidervish IA, Lasser-Ross N, Gutnick MJ, Ross WN (2010) Na+ imaging reveals little difference in action potential-evoked $\mathrm{Na}+$ influx between axon and soma. Nat Neurosci 13:852-860

Flores-Otero J, Xue HZ, Davis RL (2007) Reciprocal regulation of presynaptic and postsynaptic proteins in bipolar spiral ganglion neurons by neurotrophins. J Neurosci 27:14023-14034

Fourcaud-Trocmé N, Hansel D, van Vreeswijk C, Brunel N (2003) How spike generation mechanisms determine the neuronal response to fluctuating inputs. J Neurosci 23:11628-11640

Frank T, Khimich D, Neef A, Moser T (2009) Mechanisms contributing to synaptic $\mathrm{Ca} 2+$ signals and their heterogeneity in hair cells. Proc Natl Acad Sci USA 106:4483-4488

Frank T, Rutherford MA, Strenzke N, Neef A, Pangršič T, Khimich D, Fejtova A, Fetjova A, Gundelfinger ED, Liberman MC, Harke B, Bryan KE, Lee A, Egner A, Riedel D, Moser T (2010) Bassoon and the synaptic ribbon organize $\mathrm{Ca}^{2}+$ channels and vesicles to add release sites and promote refilling. Neuron 68:724-738

Gaffield MA, Rizzoli SO, Betz WJ (2006) Mobility of synaptic vesicles in different pools in resting and stimulated frog motor nerve terminals. Neuron 51:317-325

Gai Y, Doiron B, Kotak V, Rinzel J (2009) Noise-gated encoding of slow inputs by auditory brain stem neurons with a low-threshold K+ current. J Neurophysiol 


\section{2:3447-3460}

Gelfand S (2004) Hearing: An Introduction to Psychological and Physiological Acoustics, Fourth Edition, Revised and Expanded 4th ed. Marcel Dekker, New York.

Gersdorff von H, Borst JGG (2002) Short-term plasticity at the calyx of held. Nat Rev Neurosci 3:53-64

Gillespie D (1976) A general method for numerically simulating the stochastic time evolution of coupled chemical reactions. J. Comput. Phys 22:403-434

Girod R, Corrèges P, Jacquet J, Dunant Y (1993) Space and time characteristics of transmitter release at the nerve-electroplaque junction of Torpedo. J Physiol (Lond) 471:129-157

Glowatzki E, Fuchs PA (2002) Transmitter release at the hair cell ribbon synapse. Nat Neurosci 5:147-154

Goutman JD, Glowatzki E (2007) Time course and calcium dependence of transmitter release at a single ribbon synapse. Proc Natl Acad Sci USA 104:16341-16346

Goutman JD, Glowatzki E (2011) Short-term facilitation modulates size and timing of the synaptic response at the inner hair cell ribbon synapse. J Neurosci 31:79747981

Grant L, Yi E, Dietz J, Glowatzki E (2009) Maturation of Synaptic Transmission at the Inner Hair Cell Afferent Synapse In ARO 2009, p. 1. Available at: http://www.aro.org/archives/2009/2009_260_747a01e8.html.

Grant L, Yi E, Glowatzki E (2010) Two modes of release shape the postsynaptic response at the inner hair cell ribbon synapse. J Neurosci 30:4210-4220

Grant L, Yi E, Goutman JD, Glowatzki E (2011) Postsynaptic recordings at afferent dendrites contacting cochlear inner hair cells: monitoring multivesicular release at a ribbon synapse. J Vis Exp

Groff JA, Liberman MC (2003) Modulation of cochlear afferent response by the lateral olivocochlear system: activation via electrical stimulation of the inferior colliculus. J Neurophysiol 90:3178-3200

Grothe B, Pecka M, McAlpine D (2010) Mechanisms of Sound Localization in Mammals. Physiol Rev 90:983-1012

Grubb MS, Shu Y, Kuba H, Rasband MN, Wimmer VC, Bender KJ (2011) Short- and Long-Term Plasticity at the Axon Initial Segment. J Neurosci 31:16049-16055

Hackney CM, Mahendrasingam S, Penn A, Fettiplace R (2005) The concentrations of calcium buffering proteins in mammalian cochlear hair cells. J Neurosci 25:78677875

Harnischfeger G (1980) Brainstem units of echolocating bats code binaural time differences in the microsecond range. Naturwissenschaften 67:314-316

Haucke V, Neher E, Sigrist SJ (2011) Protein scaffolds in the coupling of synaptic 
exocytosis and endocytosis. Nat Rev Neurosci 12:127-138

He L, Wu X-S, Mohan R, Wu L-G (2006) Two modes of fusion pore opening revealed by cell-attached recordings at a synapse. Nature 444:102-105

He L, Xue L, Xu J, McNeil B, Bai L, Melicoff E, Adachi R, Wu L (2009) Compound vesicle fusion increases quantal size and potentiates synaptic transmission. Nature

Heil P, Neubauer H, Irvine DRF, Brown M (2007) Spontaneous activity of auditorynerve fibers: insights into stochastic processes at ribbon synapses. J Neurosci 27:8457-8474

Heil P, Neubauer H, Irvine DRF (2011) An improved model for the rate-level functions of auditory-nerve fibers. J Neurosci 31:15424-15437

Heil P, Neubauer H (2003) A unifying basis of auditory thresholds based on temporal summation. Proc Natl Acad Sci USA 100:6151-6156

Herz AVM, Gollisch T, Machens CK, Jaeger D (2006) Modeling single-neuron dynamics and computations: a balance of detail and abstraction. Science 314:80-85

Hess P, Lansman JB, Tsien RW (1984) Different modes of Ca channel gating behaviour favoured by dihydropyridine Ca agonists and antagonists. Nature 311:538-544

Hodgkin AL (1948) The local electric changes associated with repetitive action in a nonmedullated axon. J Physiol (Lond) 107:165-181

Hodgkin AL, Huxley AF (1952) A quantitative description of membrane current and its application to conduction and excitation in nerve. J Physiol (Lond) 117:500-544

Holt M, Cooke A, Neef A, Lagnado L (2004) High mobility of vesicles supports continuous exocytosis at a ribbon synapse. Curr Biol 14:173-183

Honeycutt R (1992) Stochastic Runge-Kutta algorithms. I. White noise. Phys Rev A 45:600-603

Hossain WA, Antic SD, Yang Y, Rasband MN, Morest DK (2005) Where is the spike generator of the cochlear nerve? Voltage-gated sodium channels in the mouse cochlea. J Neurosci 25:6857-6868

Hu W, Tian C, Li T, Yang M, Hou H, Shu Y (2009) Distinct contributions of Na(v)1.6 and $\mathrm{Na}(\mathrm{v}) 1.2$ in action potential initiation and backpropagation. Nat Neurosci 12:996-1002

Hudspeth AJ (2008) Making an effort to listen: mechanical amplification in the ear. Neuron 59:530-545

Hughes J (1946) The threshold of audition for short periods of stimulation. Proceedings of the Royal Society of London. Series B, Biological Sciences:486-490

Issa NP, Hudspeth AJ (1994) Clustering of Ca2+ channels and $\mathrm{Ca}(2+)$-activated $\mathrm{K}+$ channels at fluorescently labeled presynaptic active zones of hair cells. Proc Natl Acad Sci USA 91:7578-7582

Izhikevich EM (2007) Dynamical systems in neuroscience. The MIT Press. 
Jackman SL, Choi S-Y, Thoreson WB, Rabl K, Bartoletti TM, Kramer RH (2009) Role of the synaptic ribbon in transmitting the cone light response. Nat Neurosci 12:303310

Jackson MB, Chapman ER (2008) The fusion pores of $\mathrm{Ca}(2+)$-triggered exocytosis. Nat Struct Mol Biol 15:684-689

Jahn R, Lang T, Südhof TC (2003) Membrane fusion. Cell 112:519-533

Jahn R, Scheller RH (2006) SNAREs-engines for membrane fusion. Nat Rev Mol Cell Biol 7:631-643

Jarsky T, Tian M, Singer JH (2010) Nanodomain control of exocytosis is responsible for the signaling capability of a retinal ribbon synapse. J Neurosci 30:11885-11895

Jarsky T, Cembrowski M, Logan SM, Kath WL, Riecke H, Demb JB, Singer JH (2011) A synaptic mechanism for retinal adaptation to luminance and contrast. J Neurosci 31:11003-11015

Johnson DH (1980) The relationship between spike rate and synchrony in responses of auditory-nerve fibers to single tones. J Acoust Soc Am 68:1115-1122

Johnson SL, Forge A, Knipper M, Münkner S, Marcotti W (2008) Tonotopic variation in the calcium dependence of neurotransmitter release and vesicle pool replenishment at mammalian auditory ribbon synapses. J Neurosci 28:7670-7678

Johnson SL, Beurg M, Marcotti W, Fettiplace R (2011) Prestin-Driven Cochlear Amplification Is Not Limited by the Outer Hair Cell Membrane Time Constant. Neuron 70:1143-1154

Jonas P, Major G, Sakmann B (1993) Quantal components of unitary EPSCs at the mossy fibre synapse on CA3 pyramidal cells of rat hippocampus. J Physiol (Lond) 472:615-663

Kandel E, Schwartz J, Jessell T (2000) Principles of Neural Science 4th ed. McGraw-Hill Medical.

Keen EC, Hudspeth AJ (2006) Transfer characteristics of the hair cell's afferent synapse. Proc Natl Acad Sci USA 103:5537-5542

Khimich D, Nouvian R, Pujol R, Tom Dieck S, Egner A, Gundelfinger ED, Moser T (2005) Hair cell synaptic ribbons are essential for synchronous auditory signalling. Nature 434:889-894

Kiang NY-S (1965) Discharge Patterns of Single Fibers in the Cat's Auditory Nerve. Research Monograph [MIT] No. 35. Cambridge, MA 1965.

Kittel RJ, Wichmann C, Rasse TM, Fouquet W, Schmidt M, Schmid A, Wagh DA, Pawlu C, Kellner RR, Willig KI, Hell SW, Buchner E, Heckmann M, Sigrist SJ (2006) Bruchpilot promotes active zone assembly, Ca2+ channel clustering, and vesicle release. Science 312:1051-1054

Knight BW (1972) Dynamics of encoding in a population of neurons. J Gen Physiol 59:734-766 
Kole MHP, Ilschner SU, Kampa BM, Williams SR, Ruben PC, Stuart GJ (2008) Action potential generation requires a high sodium channel density in the axon initial segment. Nat Neurosci 11:178-186

Kössl M, Russell IJ (1992) The phase and magnitude of hair cell receptor potentials and frequency tuning in the guinea pig cochlea. J Neurosci 12:1575-1586

Krishna BS (2002) A unified mechanism for spontaneous-rate and first-spike timing in the auditory nerve. Journal of Computational Neuroscience 13:71-91

Krishna BS (2006) Comment on "Auditory-nerve first-spike latency and auditory absolute threshold: a computer model" [J. Acoust. Soc. Am. 119, 406-417 (2006)]. J Acoust Soc Am 120:591-593

Kuba H, Ishii TM, Ohmori H (2006) Axonal site of spike initiation enhances auditory coincidence detection. Nature 444:1069-1072

Kuba H, Oichi Y, Ohmori H (2010) Presynaptic activity regulates $\mathrm{Na}(+)$ channel distribution at the axon initial segment. Nature 465:1075-1078

Lapicque L (1907) Recherches quantitatives sur l'excitation électrique des nerfs traitée comme une polarisation. J. Physiol. Pathol. Gen 9:620-635

Lenzi D, Crum J, Ellisman MH, Roberts WM (2002) Depolarization redistributes synaptic membrane and creates a gradient of vesicles on the synaptic body at a ribbon synapse. Neuron 36:649-659

Lenzi D, Gersdorff von H (2001) Structure suggests function: the case for synaptic ribbons as exocytotic nanomachines. Bioessays 23:831-840

Li G-L, Keen E, Andor-Ardó D, Hudspeth AJ, Gersdorff von H (2009) The unitary event underlying multiquantal EPSCs at a hair cell's ribbon synapse. J Neurosci 29:7558-7568

Liberman MC (1978) Auditory-nerve response from cats raised in a low-noise chamber. J Acoust Soc Am 63:442-455

Liberman MC (1980) Efferent synapses in the inner hair cell area of the cat cochlea: an electron microscopic study of serial sections. Hear Res 3:189-204

Liberman MC (1982) Single-neuron labeling in the cat auditory nerve. Science 216:12391241

Liberman MC, Kiang NY (1978a) Acoustic trauma in cats. Cochlear pathology and auditory-nerve activity. Acta Otolaryngol Suppl 358:1-63

Liberman MC, Kiang NY (1978b) Acoustic trauma in cats. Cochlear pathology and auditory-nerve activity. Acta Otolaryngol Suppl 358:1-63

Lin K-H, Oleskevich S, Taschenberger H (2011) Presynaptic Ca2+ influx and vesicle exocytosis at the mouse endbulb of Held: a comparison of two auditory nerve terminals. J Physiol (Lond) 589:4301-4320

Lin X (1997) Action potentials and underlying voltage-dependent currents studied in 
cultured spiral ganglion neurons of the postnatal gerbil. Hear Res 108:157-179

Liu Q, Davis RL (2007) Regional specification of threshold sensitivity and response time in CBA/CaJ mouse spiral ganglion neurons. J Neurophysiol 98:2215-2222

LoGiudice L, Matthews G (2009) The role of ribbons at sensory synapses. The Neuroscientist 15:380-391

Luby-Phelps K (2000) Cytoarchitecture and physical properties of cytoplasm: volume, viscosity, diffusion, intracellular surface area. Int Rev Cytol 192:189-221

Luby-Phelps K, Castle PE, Taylor DL, Lanni F (1987) Hindered diffusion of inert tracer particles in the cytoplasm of mouse 3T3 cells. Proc Natl Acad Sci USA 84:49104913

Lv P, Wei D, Yamoah EN (2010) Kv7-type channel currents in spiral ganglion neurons: involvement in sensorineural hearing loss. J Biol Chem 285:34699-34707

Markram H, Wang Y, Tsodyks M (1998) Differential signaling via the same axon of neocortical pyramidal neurons. Proc Natl Acad Sci USA 95:5323-5328

Markram H, Toledo-Rodriguez M, Wang Y, Gupta A, Silberberg G, Wu C (2004) Interneurons of the neocortical inhibitory system. Nat Rev Neurosci 5:793-807

Matthews G, Fuchs P (2010) The diverse roles of ribbon synapses in sensory neurotransmission. Nat Rev Neurosci 11:812-822

Matthews G, Sterling P (2008) Evidence that vesicles undergo compound fusion on the synaptic ribbon. J Neurosci 28:5403-5411

Matveev V, Sherman A, Zucker RS (2002) New and corrected simulations of synaptic facilitation. Biophys J 83:1368-1373

Matveev V, Bertram R, Sherman A (2011) Calcium cooperativity of exocytosis as a measure of $\mathrm{Ca}^{2}+$ channel domain overlap. Brain Res 1398:126-138

McGinley MJ, Oertel D (2006) Rate thresholds determine the precision of temporal integration in principal cells of the ventral cochlear nucleus. Hear Res 216-217:5263

Meddis R (2006) Reply to comment on "Auditory-nerve first-spike latency and auditory absolute threshold: a computer model". J Acoust Soc Am 120:1192-1193

Mennerick S, Que J, Benz A, Zorumski CF (1995) Passive and synaptic properties of hippocampal neurons grown in microcultures and in mass cultures. J Neurophysiol $73: 320-332$

Merchan-Perez A, Liberman MC (1996) Ultrastructural differences among afferent synapses on cochlear hair cells: correlations with spontaneous discharge rate. J Comp Neurol 371:208-221

Meyer AC, Frank T, Khimich D, Hoch G, Riedel D, Chapochnikov NM, Yarin YM, Harke B, Hell SW, Egner A, Moser T (2009) Tuning of synapse number, structure and function in the cochlea. Nat Neurosci 12:444-453 
Millar AG, Zucker RS, Ellis-Davies GCR, Charlton MP, Atwood HL (2005) Calcium sensitivity of neurotransmitter release differs at phasic and tonic synapses. J Neurosci 25:3113-3125

Mo Z-L, Adamson CL, Davis RL (2002) Dendrotoxin-sensitive K(+) currents contribute to accommodation in murine spiral ganglion neurons. J Physiol (Lond) 542:763778

Mo ZL, Davis RL (1997a) Endogenous firing patterns of murine spiral ganglion neurons. J Neurophysiol 77:1294-1305

Mo ZL, Davis RL (1997b) Heterogeneous voltage dependence of inward rectifier currents in spiral ganglion neurons. J Neurophysiol 78:3019-3027

Moiseff A, Konishi M (1981) Neuronal and behavioral sensitivity to binaural time differences in the owl. J Neurosci 1:40-48

Moller AR (2006) Hearing, Second Edition: Anatomy, Physiology, and Disorders of the Auditory System 2nd ed. Academic Press.

Moser T, Neef A, Khimich D (2006) Mechanisms underlying the temporal precision of sound coding at the inner hair cell ribbon synapse. J Physiol (Lond) 576:55-62

Moser T, Beutner D (2000) Kinetics of exocytosis and endocytosis at the cochlear inner hair cell afferent synapse of the mouse. Proc Natl Acad Sci USA 97:883-888

Naraghi M, Neher E (1997) Linearized buffered Ca2+ diffusion in microdomains and its implications for calculation of $[\mathrm{Ca} 2+]$ at the mouth of a calcium channel. $J$ Neurosci 17:6961-6973

Naud R, Marcille N, Clopath C, Gerstner W (2008) Firing patterns in the adaptive exponential integrate-and-fire model. Biol Cybern 99:335-347

Neef A, Khimich D, Pirih P, Riedel D, Wolf F, Moser T (2007) Probing the mechanism of exocytosis at the hair cell ribbon synapse. J Neurosci 27:12933-12944

Neher E (1998a) Usefulness and limitations of linear approximations to the understanding of $\mathrm{Ca}++$ signals. Cell Calcium 24:345-357

Neher E (1998b) Vesicle pools and Ca2+ microdomains: new tools for understanding their roles in neurotransmitter release. Neuron 20:389-399

Neher E (2010) What is Rate-Limiting during Sustained Synaptic Activity: Vesicle Supply or the Availability of Release Sites. Front. Syn. Neurosci. 2:144

Neher E, Sakaba T (2008) Multiple roles of calcium ions in the regulation of neurotransmitter release. Neuron 59:861-872

Neubauer H, Heil P (2008) A physiological model for the stimulus dependence of firstspike latency of auditory-nerve fibers. Brain Res 1220:208-223

Nicol MJ, Walmsley B (2002) Ultrastructural basis of synaptic transmission between endbulbs of Held and bushy cells in the rat cochlear nucleus. J Physiol (Lond) 539:713-723 
Nouvian R, Beutner D, Parsons TD, Moser T (2006) Structure and function of the hair cell ribbon synapse. J Membr Biol 209:153-165

Nouvian R, Neef J, Bulankina AV, Reisinger E, Pangršič T, Frank T, Sikorra S, Brose N, Binz T, Moser T (2011) Exocytosis at the hair cell ribbon synapse apparently operates without neuronal SNARE proteins. Nat Neurosci

Nusser Z, Cull-Candy S, Farrant M (1997) Differences in synaptic GABA(A) receptor number underlie variation in GABA mini amplitude. Neuron 19:697-709

Oertel D (1983) Synaptic responses and electrical properties of cells in brain slices of the mouse anteroventral cochlear nucleus. J Neurosci 3:2043-2053

Oertel D (1999) The role of timing in the brain stem auditory nuclei of vertebrates. Annu. Rev. Physiol. 61:497-519

Oesch NW, Diamond JS (2011) Ribbon synapses compute temporal contrast and encode luminance in retinal rod bipolar cells. Nat Neurosci

Pan B, Zucker RS (2009) A General Model of Synaptic Transmission and Short-Term Plasticity. Neuron 62:539-554

Pandey S, White MH (2002) Parameter-extraction of a two-compartment model for whole-cell data analysis. J Neurosci Methods 120:131-143

Pangrsic T, Lasarow L, Reuter K, Takago H, Schwander M, Riedel D, Frank T, Tarantino LM, Bailey JS, Strenzke N, Brose N, Müller U, Reisinger E, Moser T (2010) Hearing requires otoferlin-dependent efficient replenishment of synaptic vesicles in hair cells. Nat Neurosci 13:869-876

Parsons TD, Sterling P (2003) Synaptic ribbon. Conveyor belt or safety belt? Neuron 37:379-382

Patuzzi R, Sellick PM (1983) A comparison between basilar membrane and inner hair cell receptor potential input-output functions in the guinea pig cochlea. J Acoust Soc Am 74:1734-1741

Paul W, Yoon D (1995) Stochastic phase space dynamics with constraints for molecular systems. Phys. Rev. E 52:2076-2083

Pawlu C, DiAntonio A, Heckmann M (2004) Postfusional control of quantal current shape. Neuron 42:607-618

Peng AW, Salles FT, Pan B, Ricci AJ (2011) Integrating the biophysical and molecular mechanisms of auditory hair cell mechanotransduction. Nat Commun 2:523

Perkins RE, Morest DK (1975) A study of cochlear innervation patterns in cats and rats with the Golgi method and Nomarkski Optics. J Comp Neurol 163:129-158

Pickett JA, Edwardson JM (2006) Compound exocytosis: mechanisms and functional significance. Traffic 7:109-116

Platzer J, Engel J, Schrott-Fischer A, Stephan K, Bova S, Chen H, Zheng H, Striessnig J (2000) Congenital deafness and sinoatrial node dysfunction in mice lacking class D 
L-type Ca2+ channels. Cell 102:89-97

Prescott SA, De Koninck Y, Sejnowski TJ (2008a) Biophysical basis for three distinct dynamical mechanisms of action potential initiation. PLoS Comput Biol 4:e1000198

Prescott SA, Ratté S, De Koninck Y, Sejnowski TJ (2008b) Pyramidal neurons switch from integrators in vitro to resonators under in vivo-like conditions. J Neurophysiol 100:3030-3042

Press WH, Teukolsky SA, Vetterling WT, Flannery BP (2007) Numerical Recipes 3rd Edition: The Art of Scientific Computing 3rd ed. Cambridge University Press.

Purves D, Augustine GJ, Fitzpatrick D, Katz LC, Lamantia A-S, McNamara JO, Williams SM (2001) Neuroscience 2nd ed. Sinauer Associates Inc.

Rancz EA, Ishikawa T, Duguid I, Chadderton P, Mahon S, Häusser M (2007) Highfidelity transmission of sensory information by single cerebellar mossy fibre boutons. Nature 450:1245-1248

Rathouz M, Trussell L (1998) Characterization of outward currents in neurons of the avian nucleus magnocellularis. J Neurophysiol 80:2824-2835

Rea R, Li J, Dharia A, Levitan ES, Sterling P, Kramer RH (2004) Streamlined synaptic vesicle cycle in cone photoreceptor terminals. Neuron 41:755-766

Rizzoli SO, Betz WJ (2005) Synaptic vesicle pools. Nat Rev Neurosci 6:57-69

Rizzoli SO, Jahn R (2007) Kiss-and-run, collapse and "readily retrievable" vesicles. Traffic 8:1137-1144

Roberts WM (1993) Spatial calcium buffering in saccular hair cells. Nature 363:74-76

Roberts WM (1994) Localization of calcium signals by a mobile calcium buffer in frog saccular hair cells. J Neurosci 14:3246-3262

Roberts WM, Jacobs RA, Hudspeth AJ (1990) Colocalization of ion channels involved in frequency selectivity and synaptic transmission at presynaptic active zones of hair cells. J Neurosci 10:3664-3684

Robertson D, Paki B (2002) Role of L-type Ca2+ channels in transmitter release from mammalian inner hair cells. II. Single-neuron activity. J Neurophysiol 87:2734-2740

Rodriguez-Contreras A, Nonner W, Yamoah EN (2002) Ca2+ transport properties and determinants of anomalous mole fraction effects of single voltage-gated $\mathrm{Ca} 2+$ channels in hair cells from bullfrog saccule. J Physiol (Lond) 538:729-745

Rodriguez-Contreras A, Yamoah EN (2001) Direct measurement of single-channel $\mathrm{Ca}(2+)$ currents in bullfrog hair cells reveals two distinct channel subtypes. J Physiol (Lond) 534:669-689

Rodríguez-Contreras A, Yamoah EN (2003) Effects of permeant ion concentrations on the gating of L-type Ca2+ channels in hair cells. Biophys J 84:3457-3469

Rose JE, Brugge JF, Anderson DJ, Hind JE (1967) Phase-locked response to lowfrequency tones in single auditory nerve fibers of the squirrel monkey. J 
Neurophysiol 30:769-793

Rossi ML, Martini M, Pelucchi B, Fesce R (1994) Quantal nature of synaptic transmission at the cytoneural junction in the frog labyrinth. J Physiol (Lond) 478 (Pt 1):17-35

Rothman JS, Young ED, Manis PB (1993) Convergence of auditory nerve fibers onto bushy cells in the ventral cochlear nucleus: implications of a computational model. J Neurophysiol 70:2562-2583

Rothman JS, Manis PB (2003) The roles potassium currents play in regulating the electrical activity of ventral cochlear nucleus neurons. J Neurophysiol 89:3097-3113

Roux I, Safieddine S, Nouvian R, Grati M, Simmler M-C, Bahloul A, Perfettini I, Le Gall M, Rostaing P, Hamard G, Triller A, Avan P, Moser T, Petit C (2006) Otoferlin, defective in a human deafness form, is essential for exocytosis at the auditory ribbon synapse. Cell 127:277-289

Russell IJ, Kössl M (1991) The voltage responses of hair cells in the basal turn of the guinea-pig cochlea. J Physiol (Lond) 435:493-511

Russell IJ, Kössl M (1992) Modulation of hair cell voltage responses to tones by lowfrequency biasing of the basilar membrane in the guinea pig cochlea. J Neurosci 12:1587-1601

Russell IJ, Sellick PM (1978) Intracellular studies of hair cells in the mammalian cochlea. J Physiol (Lond) 284:261-290

Rusznák Z, Szucs G (2009) Spiral ganglion neurones: an overview of morphology, firing behaviour, ionic channels and function. Pflugers Arch 457:1303-1325

Sachs MB, Abbas PJ (1974) Rate versus level functions for auditory-nerve fibers in cats: tone-burst stimuli. J Acoust Soc Am 56:1835-1847

Safieddine S, Wenthold RJ (1999) SNARE complex at the ribbon synapses of cochlear hair cells: analysis of synaptic vesicle- and synaptic membrane-associated proteins. Eur J Neurosci 11:803-812

Sahara Y, Takahashi T (2001) Quantal components of the excitatory postsynaptic currents at a rat central auditory synapse. J Physiol (Lond) 536:189-197

Saito K (1990) Freeze-fracture organization of hair cell synapses in the sensory epithelium of guinea pig organ of Corti. J Electron Microsc Tech 15:173-186

Santos-Sacchi J (1993) Voltage-dependent ionic conductances of type I spiral ganglion cells from the guinea pig inner ear. J Neurosci 13:3599-3611

Saviane C, Silver RA (2006) Fast vesicle reloading and a large pool sustain high bandwidth transmission at a central synapse. Nature 439:983-987

Savtchenko LP, Rusakov DA (2007) The optimal height of the synaptic cleft. Proc Natl Acad Sci USA 104:1823-1828

Sätzler K, Söhl LF, Bollmann JH, Borst JGG, Frotscher M, Sakmann B, Lübke JHR (2002) Three-dimensional reconstruction of a calyx of Held and its postsynaptic 
principal neuron in the medial nucleus of the trapezoid body. J Neurosci 22:1056710579

Scepek S, Coorssen JR, Lindau M (1998) Fusion pore expansion in horse eosinophils is modulated by $\mathrm{Ca} 2+$ and protein kinase $\mathrm{C}$ via distinct mechanisms. EMBO J $17: 4340-4345$

Schmitz F (2009) The making of synaptic ribbons: how they are built and what they do. Neuroscientist 15:611-624

Schnee ME, Santos-Sacchi J, Castellano-Muñoz M, Kong J-H, Ricci AJ (2011) Calciumdependent synaptic vesicle trafficking underlies indefatigable release at the hair cell afferent fiber synapse. Neuron 70:326-338

Schneggenburger R, Sakaba T, Neher E (2002) Vesicle pools and short-term synaptic depression: lessons from a large synapse. Trends Neurosci 25:206-212

Schneggenburger R, Neher E (2000) Intracellular calcium dependence of transmitter release rates at a fast central synapse. Nature 406:889-893

Schneggenburger R, Neher E (2005) Presynaptic calcium and control of vesicle fusion. Curr Opin Neurobiol 15:266-274

Schwarz K, Natarajan S, Kassas N, Vitale N, Schmitz F (2011) The Synaptic Ribbon Is a Site of Phosphatidic Acid Generation in Ribbon Synapses. J Neurosci 31:1599616011

Segovia M, Alés E, Montes MA, Bonifas I, Jemal I, Lindau M, Maximov A, Südhof TC, Alvarez de Toledo G (2010) Push-and-pull regulation of the fusion pore by synaptotagmin-7. Proc Natl Acad Sci USA 107:19032-19037

Sewell WF (1984) The relation between the endocochlear potential and spontaneous activity in auditory nerve fibres of the cat. J Physiol (Lond) 347:685-696

Shepherd RK, Hardie NA, Baxi JH (2001) Electrical stimulation of the auditory nerve: single neuron strength-duration functions in deafened animals. Ann Biomed Eng 29:195-201

Shu Y, Yu Y, Yang J, McCormick DA (2007a) Selective control of cortical axonal spikes by a slowly inactivating K+ current. Proc Natl Acad Sci USA 104:11453-11458

Shu Y, Duque A, Yu Y, Haider B, McCormick DA (2007b) Properties of action-potential initiation in neocortical pyramidal cells: evidence from whole cell axon recordings. J Neurophysiol 97:746-760

Siegel JH (1992) Spontaneous synaptic potentials from afferent terminals in the guinea pig cochlea. Hear Res 59:85-92

Siegel JH, Relkin EM (1987) Antagonistic effects of perilymphatic calcium and magnesium on the activity of single cochlear afferent neurons. Hear Res 28:131147

Simmons D, Bertolotto C, Leong M (1995) Synaptic ultrastructure within the amphibian papilla of Rana pipiens pipiens: rostrocaudal differences. Auditory Neuroscience 


\section{$1: 183-193$}

Singer JH, Lassová L, Vardi N, Diamond JS (2004) Coordinated multivesicular release at a mammalian ribbon synapse. Nat Neurosci 7:826-833

Snellman J, Mehta B, Babai N, Bartoletti TM, Akmentin W, Francis A, Matthews G, Thoreson W, Zenisek D (2011) Acute destruction of the synaptic ribbon reveals a role for the ribbon in vesicle priming. Nat Neurosci

Soekmadji C, Thorn P (2010) Secretory control: evidence for agonist regulation of postfusion vesicle behaviour. Clin. Exp. Pharmacol. Physiol. 37:218-221

Staal RGW, Mosharov EV, Sulzer D (2004) Dopamine neurons release transmitter via a flickering fusion pore. Nat Neurosci 7:341-346

Stein RB (1967) Some models of neuronal variability. Biophys J 7:37-68

Strenzke N, Chanda S, Kopp-Scheinpflug C, Khimich D, Reim K, Bulankina AV, Neef A, Wolf F, Brose N, Xu-Friedman MA, Moser T (2009) Complexin-I is required for high-fidelity transmission at the endbulb of Held auditory synapse. J Neurosci 29:7991-8004

Sumner CJ, Lopez-Poveda EA, O'Mard LP, Meddis R (2002) A revised model of the inner-hair cell and auditory-nerve complex. J Acoust Soc Am 111:2178-2188

Suryanarayanan A, Slaughter MM (2006) Synaptic transmission mediated by internal calcium stores in rod photoreceptors. J Neurosci 26:1759-1766

Südhof TC (2004) The synaptic vesicle cycle. Annu. Rev. Neurosci. 27:509-547

Szabó ZS, Harasztosi CS, Sziklai I, Szûcs G, Rusznák Z (2002) Ionic currents determining the membrane characteristics of type I spiral ganglion neurons of the guinea pig. Eur J Neurosci 16:1887-1895

Sørensen JB (2009) Conflicting views on the membrane fusion machinery and the fusion pore. Annu. Rev. Cell Dev. Biol. 25:513-537

Taberner AM, Liberman MC (2005) Response properties of single auditory nerve fibers in the mouse. J Neurophysiol 93:557-569

Takahashi N, Kishimoto T, Nemoto T, Kadowaki T, Kasai H (2002) Fusion pore dynamics and insulin granule exocytosis in the pancreatic islet. Science 297:13491352

Takamori S et al. (2006) Molecular anatomy of a trafficking organelle. Cell 127:831-846

Trapani JG, Nicolson T (2011) Mechanism of spontaneous activity in afferent neurons of the zebrafish lateral-line organ. J Neurosci 31:1614-1623

Tritsch NX, Yi E, Gale JE, Glowatzki E, Bergles DE (2007) The origin of spontaneous activity in the developing auditory system. Nature 450:50-55

Trommershäuser J, Marienhagen J, Zippelius A (1999) Stochastic model of central synapses: slow diffusion of transmitter interacting with spatially distributed receptors and transporters. J Theor Biol 198:101-120 
Trussell LO (2002) Transmission at the hair cell synapse. Nat Neurosci 5:85-86

Tsodyks MV, Markram H (1997) The neural code between neocortical pyramidal neurons depends on neurotransmitter release probability. Proc Natl Acad Sci USA 94:719-723

Wadiche JI, Jahr CE (2001) Multivesicular release at climbing fiber-Purkinje cell synapses. Neuron 32:301-313

Walter AM, Groffen AJ, Sørensen JB, Verhage M (2011) Multiple Ca2+ sensors in secretion: teammates, competitors or autocrats? Trends Neurosci 34:487-497

Wang X, Thiagarajan R, Wang Q, Tewolde T, Rich MM, Engisch KL (2008) Regulation of quantal shape by Rab3A: evidence for a fusion pore-dependent mechanism. J Physiol (Lond) 586:3949-3962

Yamada WM, Zucker RS (1992) Time course of transmitter release calculated from simulations of a calcium diffusion model. Biophys J 61:671-682

Yang H, Xu-Friedman MA (2009) Impact of synaptic depression on spike timing at the endbulb of Held. J Neurophysiol 102:1699-1710

Yi E, Roux I, Glowatzki E (2010) Dendritic HCN channels shape excitatory postsynaptic potentials at the inner hair cell afferent synapse in the mammalian cochlea. J Neurophysiol 103:2532-2543

Zampini V, Johnson SL, Franz C, Lawrence ND, Münkner S, Engel J, Knipper M, Magistretti J, Masetto S, Marcotti W (2010) Elementary properties of CaV1.3 $\mathrm{Ca}(2+)$ channels expressed in mouse cochlear inner hair cells. J Physiol (Lond) 588:187-199

Zenisek D, Steyer JA, Almers W (2000) Transport, capture and exocytosis of single synaptic vesicles at active zones. Nature 406:849-854

Zhai RG, Bellen HJ (2004) The architecture of the active zone in the presynaptic nerve terminal. Physiology (Bethesda) 19:262-270

Zhou Z, Misler S, Chow RH (1996) Rapid fluctuations in transmitter release from single vesicles in bovine adrenal chromaffin cells. Biophys J 70:1543-1552

Zilany MSA, Bruce IC, Nelson PC, Carney LH (2009) A phenomenological model of the synapse between the inner hair cell and auditory nerve: long-term adaptation with power-law dynamics. J Acoust Soc Am 126:2390-2412 